\title{
Visible Light-Triggered Monofluoromethylation of Alkenes by Strongly Reducing 1,4-Bis(diphenylamino)naphthalene Photoredox Catalysis
}

\author{
Naoki Noto, ${ }^{\dagger \ddagger}$ Takashi Koike ${ }^{\star},{ }^{\dagger \ddagger}$ Munetaka Akita*†‡ \\ 'Laboratory for Chemistry and Life Science, Institute of Innovative Research \\ ‡School of Materials and Chemical Technology, Tokyo Institute of Technology \\ R1-27, 4259 Nagatsuta-cho, Midori-ku, Yokohama 226-8503, Japan
}

Table of Contents

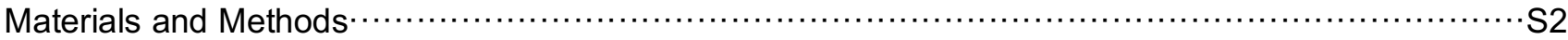

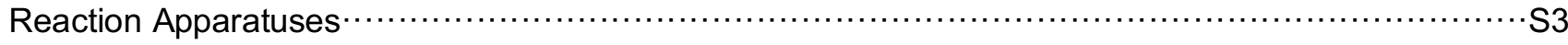

Preparation of $N^{1}, N^{1}, N^{4}, N^{4}$-Tetraphenylnaphthalene-1,4-diamine (1: Catalyst) $\cdots \cdots \cdots \cdots \cdots \cdots \cdots \cdots \cdots \cdots \cdots \cdots \cdots \cdots \cdots$

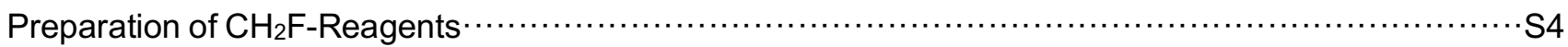

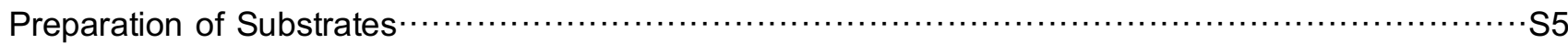

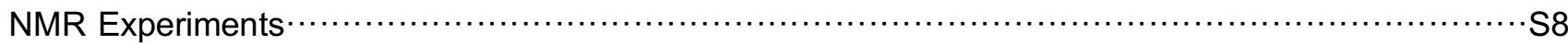

General Procedures for Hydroxy-Monofluoromethylation and Characterization $\cdots \cdots \cdots \cdots \cdots \cdots \cdots \cdots \cdots \cdots \cdots \cdots \cdots \cdots \cdots$

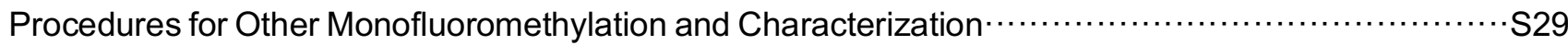

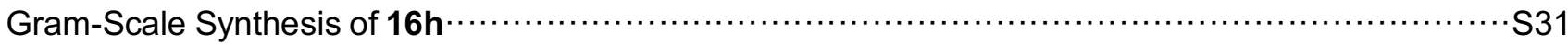

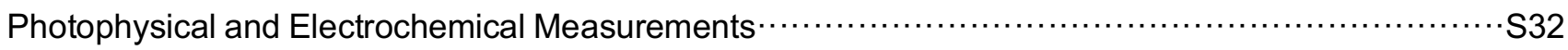

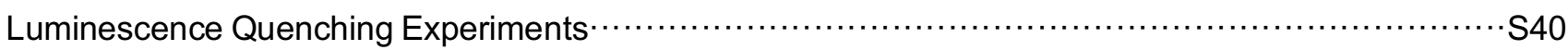

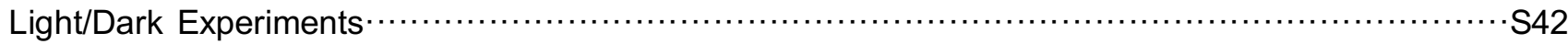

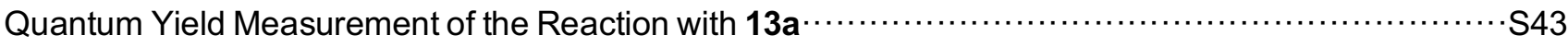

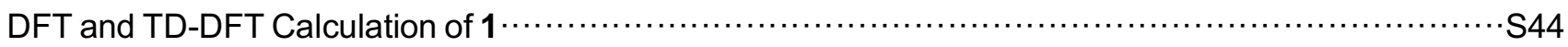

Crystallographic Data of 1 …

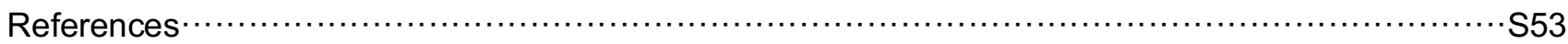

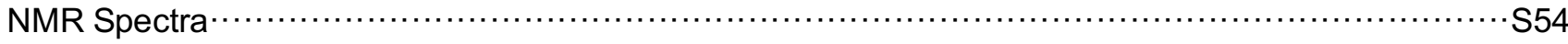




\section{Materials and Methods}

All the chemicals for synthesis of the substrates and the catalysts were commercially available. All the reactions were carried out with standard Schlenk techniques unless otherwise noted. Anhydrous acetone, $\mathrm{CH}_{3} \mathrm{CN}$, mehtanol and DMSO were purchased from Kanto Chemical Co., Inc. Anhydrous 1,2dichloroethane was purchased from Aldrich. Thin-layer chromatography was performed on TLC plates with 60 F254 (Merck). Purification was performed by flash column chromatography on silica gel (Aldrich, silica gel $60 \AA$ ). The crystallographic data were deposited at the Cambridge Crystallographic Data Centre: 1 (CCDC 1880269). Experiments and measurements were carried out with the following apparatuses.

Visible light irradiation: Relyon LED lamp $(3 \mathrm{~W} \times 2: \lambda=425 \mathrm{~nm}, 3 \mathrm{~W} \times 2: \lambda=380 \mathrm{~nm})$. NMR spectra: Bruker AVANCE-400 (400 MHz for ${ }^{1} \mathrm{H}$ NMR) and Bruker AVANCE-500 (500 MHz for ${ }^{1} \mathrm{H}$ NMR) (Reference of ${ }^{1} \mathrm{H}$ NMR spectra: Residual protio impurities in the deuterated solvents. Reference of ${ }^{19} \mathrm{~F}$ NMR spectra: Trifluoroacetic acid (-76.55 ppm). HRMS (ESI-TOF): Bruker micrOTOF II. Recycling preparative HPLC: Japan Analytical Industry Co., Ltd. (JAI) LC-9225. (Column: JAIGEL-1H-40 and JAIGEL-2H-40), and Japan Analytical Industry Co., Ltd. (JAI) LC-9201. (Column: JAIGEL-1H-20 and JAIGEL-2H-20). UV-vis: JASCO V-670DS. Fluorescence: HITACHI F-7000. Absolute PL quantum yield: Hamamatsu Photonics C9920-02G with an integration sphere. CV: Hokutodenkou HZ-5000. Excited-state lifetime: Hamamatsu Photonics C7700-ABS-N. Elemental analysis: J-SCIENCE JM10. Single-crystal X-ray measurement: Bruker SMART APEX II ULTRA. GC-MS: SHIMADZU GC-2010/PARVUM2 (Column: Rxi-5ms) 


\section{Reaction Apparatuses}

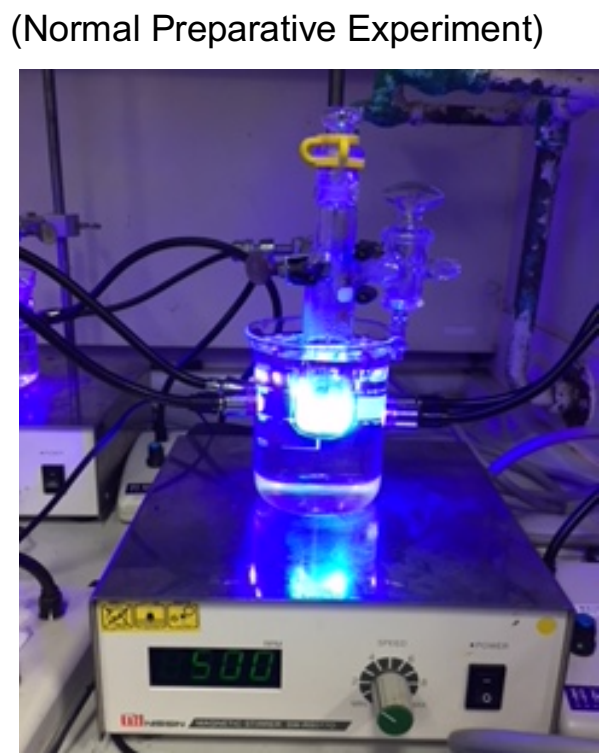

(Gram-Scale Experiment)

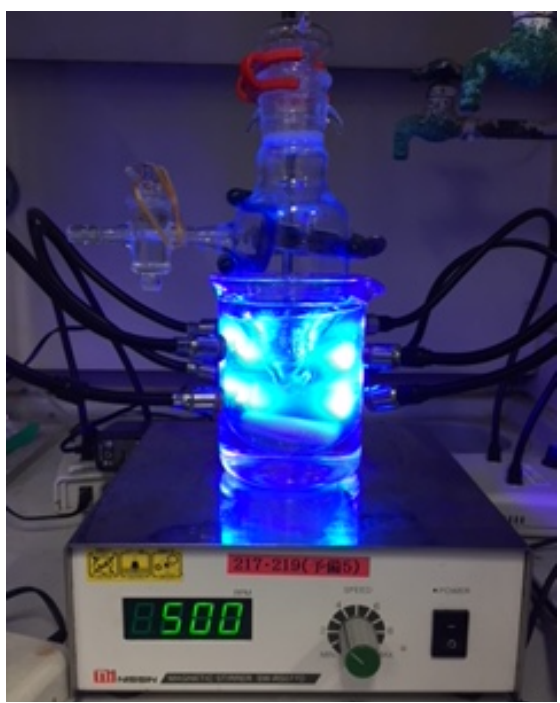

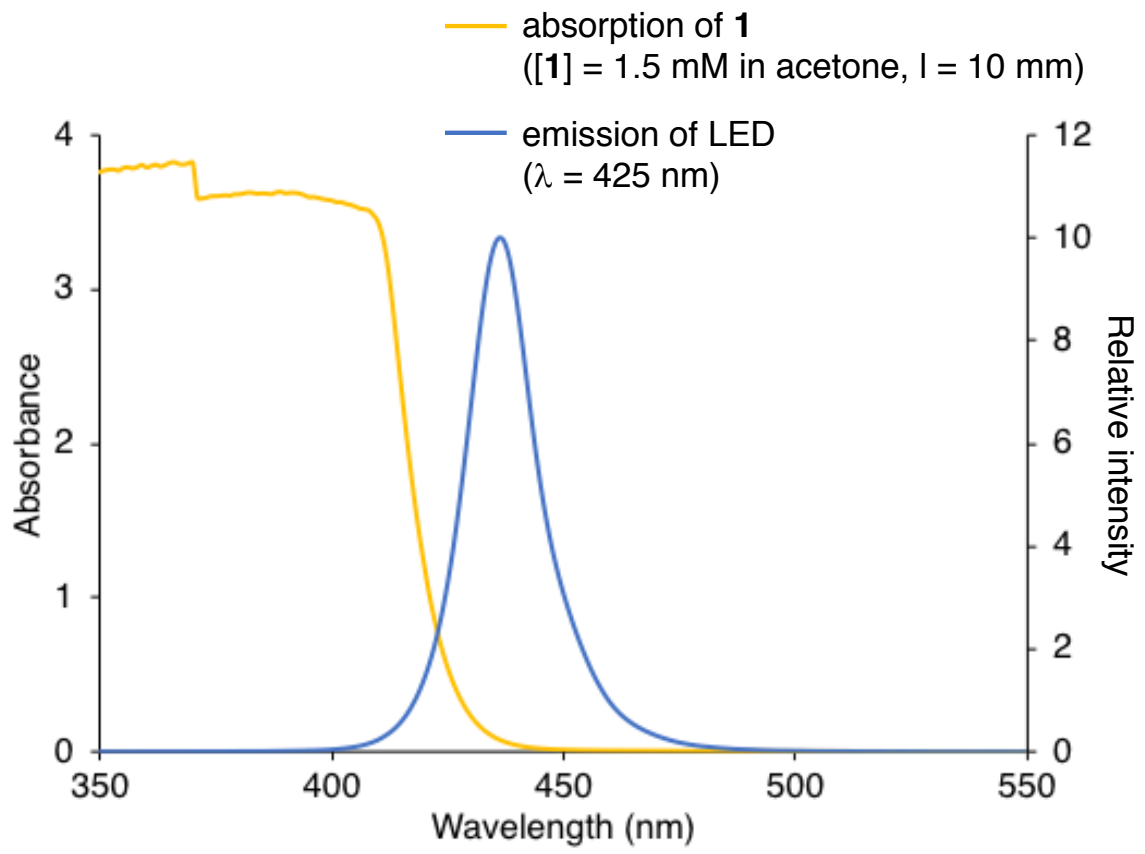

Figure S1. UV-vis spectrum of 1 and output spectrum of a Relyon LED lamp. 


\section{Preparation of $N^{1}, N^{1}, N^{4}, N^{4}$-Tetraphenylnaphthalene-1,4-diamine (1: Catalyst)}<smiles>Brc1cccc2c(Br)cccc12</smiles>
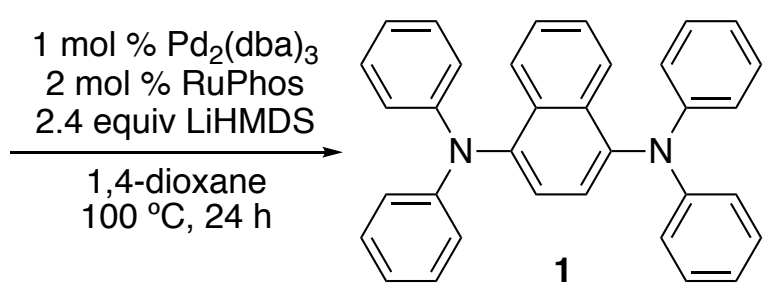

The synthetic methodology for catalysts was referred to our previous report. ${ }^{1}$ To 1,4 -dibromonaphthalene (1.14 g, $4.00 \mathrm{mmol})$, diphenylamine (1.62 g, $9.60 \mathrm{mmol})$, LiHMDS (1.61 g, $9.61 \mathrm{mmol}), \mathrm{Pd}_{2}\left(\mathrm{dba}_{3}(36.6\right.$ $\mathrm{mg}, 0.0400 \mathrm{mmol}$ ) and RuPhos ( $37.3 \mathrm{mg}, 0.0799 \mathrm{mmol}$ ) placed in a two-neck flask was added 1,4-dioxane $(40 \mathrm{~mL})$ under $\mathrm{N}_{2}$ atmosphere, and the mixture was stirred at $100^{\circ} \mathrm{C}$ for $24 \mathrm{~h}$. After cooling it to room temperature, precipitates were filtered off and washed with $\mathrm{CH}_{2} \mathrm{Cl}_{2}$, and the volatiles were evaporated. The residue was filtered through a silica-gel pad with $\mathrm{CH}_{2} \mathrm{Cl}_{2}$ as an eluent. After concentration under reduced pressure, the obtained solid was purified by recrystallization with $\mathrm{CH}_{2} \mathrm{Cl}_{2}$ and $\mathrm{MeOH}$. Then, 1 was obtained as a pale yellow solid $(1.57 \mathrm{~g}, 3.38 \mathrm{mmol}, 85 \%)$. Recrystallization from $\mathrm{CH}_{2} \mathrm{Cl}_{2} / \mathrm{MeOH}$ afforded crystals, which are suitable for single-crystal X-ray structure analysis.

${ }^{1} \mathrm{H}$ NMR (400 MHz, $\mathrm{CDCl}_{3}, \mathrm{rt}$ ): $\delta 8.00$ (dd, $J=6.5 \mathrm{~Hz}, 3.3 \mathrm{~Hz}, 2 \mathrm{H}$; naphthalene's $A r$ ), 7.34 (dd, $J=6.5 \mathrm{~Hz}$, $3.3 \mathrm{~Hz}, 2 \mathrm{H}$; naphthalene's $A r), 7.32$ (s, 2H; naphthalene's $A r), 7.25-7.21(8 \mathrm{H} ; A r), 7.07-7.05(8 \mathrm{H} ; A r), 6.96$ (t, $J=7.3 \mathrm{~Hz}, 4 \mathrm{H} ; A r)$.

${ }^{13} \mathrm{C}$ NMR $\left(125 \mathrm{MHz}, \mathrm{CDCl}_{3}\right.$, rt): $\delta 148.4,142.3,133.3,129.5,128.2,126.9,125.2,122.3,122.2$.

HRMS (ESI-TOF) calcd m/z for $\left[\mathrm{C}_{34} \mathrm{H}_{26} \mathrm{~N}_{2}\right]^{+} 462.2091$ found 462.2091.

EA: calculated for $\mathrm{C}_{34} \mathrm{H}_{26} \mathrm{~N}_{2}$ : C, 88.28; $\mathrm{H}, 5.67 ; \mathrm{N}, 6.06$, found: $\mathrm{C}, 88.32 ; \mathrm{H}, 5.54 ; \mathrm{N}, 6.04$.

Melting point: $188^{\circ} \mathrm{C}$.

\section{Preparation of $\mathrm{CH}_{2} \mathrm{~F}$-Reagents}

Fluoromethyl phenyl sulfone 8 was purchased from TCl. S-Monofluoromethyl-S-phenyl-2,3,4,5tetramethylphenylsulfonium tetrafluoroborate $\mathbf{5}$ was prepared by the procedure reported previously.2a,b

\section{Preparation of 3}

4-Methyl- $N$-(methyl(oxo)(phenyl)- $\lambda^{6}$-sulfaneylidene)benzenesulfonamide

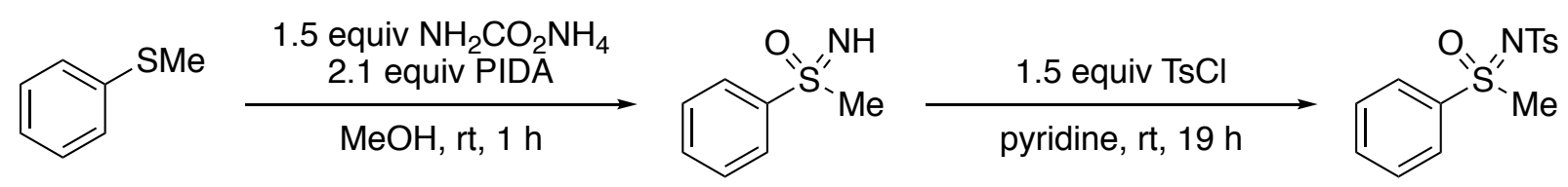

Synthesis was carried out by the modified procedures according to the reported literature. ${ }^{2 \mathrm{C}}$ To a solution of thioanisol ( $4.97 \mathrm{~g}, 40.0 \mathrm{mmol})$, ammonium carbamate $(4.68 \mathrm{~g}, 59.9 \mathrm{mmol})$ in $\mathrm{MeOH}(150 \mathrm{~mL})$ was added iodobenzene diacetate (PIDA) $(27.1 \mathrm{~g}, 84.1 \mathrm{mmol})$ under air. The mixture was stirred at room temperature for $1 \mathrm{~h}$. Then, the volatiles were removed under reduced pressure, and imino(methyl)(phenyl)- $\lambda^{6}$-sulfanone was obtained as a yellow oil after purification by flash column chromatography on silica-gel (ethyl acetate/methanol $=9: 1$ ). 
Next, the obtained oil was transferred to a $100 \mathrm{~mL}$ of two-neck flask, and to a solution of it in pyridine (20 $\mathrm{mL}$ ) was added $p$-toluenesulfonyl chloride at $0{ }^{\circ} \mathrm{C}$ under $\mathrm{N}_{2}$ atmosphere. The mixture was stirred at room temperature for $19 \mathrm{~h}$. The desired product was obtained as a brown solid $(9.92 \mathrm{~g}, 32.1 \mathrm{mmol}, 80 \%)$ after purification by flash column chromatography on silica-gel (hexane/ethyl acetate $=2: 1$ ) and reprecipitation with ethyl acetate and hexane.

Spectral data is in agreement with the reported literature. ${ }^{2 \mathrm{~d}}$

${ }^{1} \mathrm{H}$ NMR $\left(400 \mathrm{MHz}, \mathrm{CDCl}_{3}, \mathrm{rt}\right): \delta 8.02(\mathrm{~d}, J=7.4 \mathrm{~Hz}, 2 \mathrm{H}), 7.86(\mathrm{~d}, J=8.3 \mathrm{~Hz}, 2 \mathrm{H}), 7.70(\mathrm{t}, J=7.4 \mathrm{~Hz}, 1 \mathrm{H})$, 7.60 (t, J = 8.0 Hz, 2H), 7.26 (d, J = 8.0 Hz, 2H), 3.43 (s, 3H), 2.40 (s, 3H).

$N$-((Fluoromethyl)(oxo)(phenyl)- $\lambda^{6}$-sulfaneylidene)-4-methylbenzenesulfonamide (3)

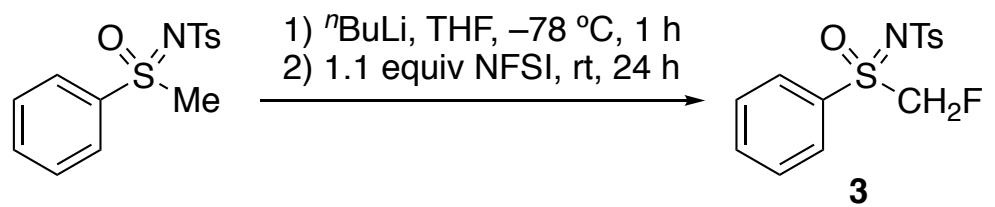

Synthesis was carried out by the procedures according to the reported literature. ${ }^{2 d}$ To a solution of 4methyl- $N$-(methyl(oxo)(phenyl)- $\lambda^{6}$-sulfaneylidene)benzenesulfonamide $(9.92 \mathrm{~g}, 32.1 \mathrm{mmol})$ in THF (150 $\mathrm{mL}$ ) was added ${ }^{n} \mathrm{BuLi}(1.57 \mathrm{M}$ in hexane, $21.5 \mathrm{~mL}, 33.8 \mathrm{mmol})$ at $-78^{\circ} \mathrm{C}$ under $\mathrm{N}_{2}$ atmosphere. After the mixture was stirred at the same temperature for $1 \mathrm{~h}, \mathrm{~N}$-fluorobenzenesulfonimide (NFSI) (11.1 g, 35.3 mmol) was added at $-78^{\circ} \mathrm{C}$. The mixture was stirred at room temperature for $24 \mathrm{~h}$. Then, saturated $\mathrm{NH}_{4} \mathrm{Cl}$ aq. was added and the water phase was extracted with ethyl acetate. The organic layer was washed with brine, dried over $\mathrm{MgSO}_{4}$, filtered and removed under reduced pressure. The desired product 3 was obtained as a white solid (6.06 g, $18.5 \mathrm{mmol}, 58 \%)$ after purification by flash column chromatography on silica-gel (hexane/ethyl acetate $=3: 1$ ) and reprecipitation with ethyl acetate and hexane.

Spectral data is in agreement with the reported literature. ${ }^{2 d}$

${ }^{1} \mathrm{H}$ NMR $\left(400 \mathrm{MHz}, \mathrm{CDCl}_{3}, \mathrm{rt}\right): \delta 8.03(\mathrm{~d}, J=7.6 \mathrm{~Hz}, 2 \mathrm{H}), 7.90(\mathrm{~d}, J=8.3 \mathrm{~Hz}, 2 \mathrm{H}), 7.76(\mathrm{t}, J=7.6 \mathrm{~Hz}, 1 \mathrm{H})$, $7.63(\mathrm{t}, J=7.8 \mathrm{~Hz}, 2 \mathrm{H}), 7.29(\mathrm{~d}, J=8.2 \mathrm{~Hz}, 2 \mathrm{H}), 5.71(\mathrm{dd}, J=76.6,9.3 \mathrm{~Hz}, 1 \mathrm{H}), 5.60$ (dd, $J=77.1,9.3$ $\mathrm{Hz}, 1 \mathrm{H}), 2.41(\mathrm{~s}, 3 \mathrm{H})$.

\section{Preparation of Substrates}

Substrates except commercially available ones were synthesized as follows. Substrates were prepared through Wittig reaction.

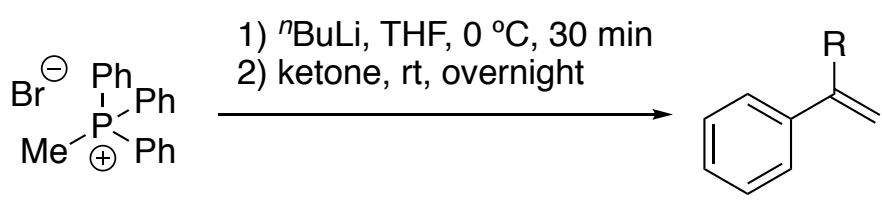

To a suspension of methyltriphenylphosphonium bromide (1 equiv) in THF was added ${ }^{n} \mathrm{BuLi}$ (1 equiv) at 0 ${ }^{\circ} \mathrm{C}$ under $\mathrm{N}_{2}$ atmosphere. After the mixture was stirred at the same temperature for $30 \mathrm{~min}$, a corresponding ketone ( 1 equiv) was added at $0{ }^{\circ} \mathrm{C}$. The mixture was stirred at room temperature overnight. Water was 
added to the mixture, and the water phase was extracted with $\mathrm{Et}_{2} \mathrm{O}$. The organic layer was dried over $\mathrm{MgSO}_{4}$, filtered and removed under reduced pressure. The desired product was obtained after purification by flash column chromatography on silica-gel (pentane).

4-(1-Phenylvinyl)-1,1'-biphenyl (15e)<smiles>C=C(c1ccccc1)c1ccc(-c2ccccc2)cc1</smiles>

According to the above procedure, methyltriphenylphosphonium bromide $(5.72 \mathrm{~g}, 16.0 \mathrm{mmol}),{ }^{n} \mathrm{BuLi}(2.76$ $\mathrm{M}$ in hexane, $5.80 \mathrm{~mL}, 16.0 \mathrm{mmol})$ and 4-phenylbenzophenone $(4.13 \mathrm{~g}, 16.0 \mathrm{mmol})$ afforded $15 \mathrm{e}$ as a white solid $(2.50 \mathrm{~g}, 9.76 \mathrm{mmol}, 61 \%)$.

Spectral data is in agreement with the reported literature. ${ }^{3 a}$

${ }^{1} \mathrm{H}$ NMR (400 MHz, $\left.\mathrm{CDCl}_{3}, \mathrm{rt}\right): \delta 7.63(\mathrm{~d}, J=7.5 \mathrm{~Hz}, 2 \mathrm{H}), 7.58(\mathrm{~d}, J=8.2 \mathrm{~Hz}, 2 \mathrm{H}), 7.47-7.33(10 \mathrm{H}), 5.53$ (s, 1H), $5.48(\mathrm{~s}, 1 \mathrm{H})$.

2-(1-Phenylvinyl)thiophene (15I)<smiles>C=C(c1ccccc1)c1cccs1</smiles>

15I

According to the above procedure, methyltriphenylphosphonium bromide $(5.70 \mathrm{~g}, 16.0 \mathrm{mmol}),{ }^{n} \mathrm{BuLi}(2.76$ $\mathrm{M}$ in hexane, $5.80 \mathrm{~mL}, 16.0 \mathrm{mmol})$ and 2-benzoylthiophene $(3.01 \mathrm{~g}, 16.0 \mathrm{mmol})$ afforded $15 \mathrm{I}$ as a colorless oil ( $2.48 \mathrm{~g}, 13.3 \mathrm{mmol}, 83 \%)$.

Spectral data is in agreement with the reported literature. ${ }^{3 \mathrm{~b}}$

${ }^{1} \mathrm{H}$ NMR (400 MHz, $\left.\mathrm{CDCl}_{3}, \mathrm{rt}\right): \delta 7.45-7.35(5 \mathrm{H}), 7.24(\mathrm{dd}, J=5.1,1.0 \mathrm{~Hz}, 1 \mathrm{H}), 6.98(\mathrm{dd}, J=5.1,3.6 \mathrm{~Hz}$, $1 \mathrm{H}), 6.91(\mathrm{dd}, J=3.6,1.0 \mathrm{~Hz}, 1 \mathrm{H}), 5.59(\mathrm{~s}, 1 \mathrm{H}), 5.25(\mathrm{~s}, 1 \mathrm{H})$. 
4-Methylene-2-phenylchromane (23)

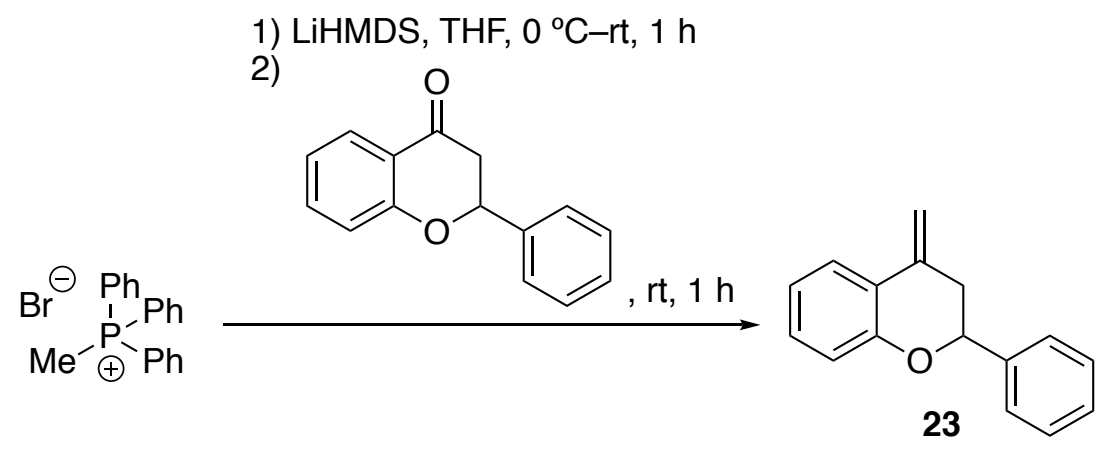

To a suspension of methyltriphenylphosphonium bromide $(4.29 \mathrm{~g}, 12.0 \mathrm{mmol})$ in THF was added LiHMDS $(1.00 \mathrm{M}$ in THF, $12.0 \mathrm{~mL}, 12.0 \mathrm{mmol})$ at $0{ }^{\circ} \mathrm{C}$ under $\mathrm{N}_{2}$ atmosphere. After being stirred at room temperature for $30 \mathrm{~min}$, the mixture was cooled to $0^{\circ} \mathrm{C}$ and flavanone $(2.24 \mathrm{~g}, 10.0 \mathrm{mmol})$ was added at $0{ }^{\circ} \mathrm{C}$. The mixture was stirred at room temperature for $1 \mathrm{~h}$. Saturated $\mathrm{NH}_{4} \mathrm{Cl}$ aq. was added to the mixture, and the water phase was separated and extracted with ethyl acetate. The organic layer was washed with brine, dried over $\mathrm{MgSO}_{4}$, filtered and the volatiles were removed under reduced pressure. 23 was obtained as a white solid $(0.453 \mathrm{~g}, 2.04 \mathrm{mmol}, 20 \%)$ after purification by flash column chromatography on silica-gel (pentane).

${ }^{1} \mathrm{H}$ NMR $\left(400 \mathrm{MHz}, \mathrm{CDCl}_{3}, \mathrm{rt}\right): \delta 7.62(\mathrm{dd}, \mathrm{J}=8.2 \mathrm{~Hz}, 1.5 \mathrm{~Hz}, 1 \mathrm{H} ; A r), 7.47-7.32(5 \mathrm{H} ; A r), 7.21(\mathrm{~m}, 1 \mathrm{H} ; A r)$, 6.96-6.92 (2H; Ar), $5.57(\mathrm{~d}, J=1.8 \mathrm{~Hz}, 1 \mathrm{H} ; \mathrm{C}=\mathrm{CHH}), 5.11\left(\mathrm{dd}, J=11.0 \mathrm{~Hz}, 3.0 \mathrm{~Hz}, 1 \mathrm{H} ; \mathrm{OCHCH}_{2} \mathrm{Ph}\right), 4.93$ $(\mathrm{d}, \mathrm{J}=1.8 \mathrm{~Hz}, 1 \mathrm{H} ; \mathrm{C}=\mathrm{CHH}), 2.92-2.78\left(2 \mathrm{H} ; \mathrm{OCHCH}_{2} \mathrm{Ph}\right)$.

${ }^{13} \mathrm{C}$ NMR $\left(125 \mathrm{MHz}, \mathrm{CDCl}_{3}\right.$, rt): $\delta 155.0,140.9,137.4,129.8,128.7,128.3,126.3,124.5,121.5,121.0$, $117.8,107.8,78.5,39.3$.

HRMS (ESI-TOF) calcd m/z for $\left[\mathrm{C}_{16} \mathrm{H}_{14} \mathrm{O}+\mathrm{Na}\right]^{+} 245.0937$ found 245.0938 . 


\section{NMR Experiments}

Typical procedures for NMR experiments

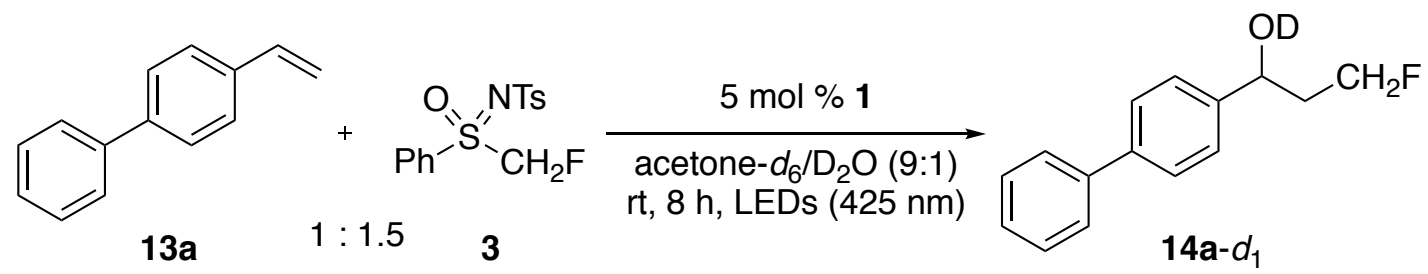

p-Vinylbiphenyl 13a (4.5 mg, $25.0 \mu \mathrm{mol}), 3$ (12.3 mg, $37.6 \mu \mathrm{mol}), 1$ (0.58 mg, $1.25 \mu \mathrm{mol})$ and tetraethylsilane were added to an NMR tube under $\mathrm{N}_{2}$ atmosphere. Then, the mixed solvent (acetone- $d_{6}$ : $0.45 \mathrm{~mL}, \mathrm{D}_{2} \mathrm{O}: 0.05 \mathrm{~mL}$ ) was added and the mixture was degassed by three freeze-pump-thaw cycles. The NMR tube was placed at $2-3 \mathrm{~cm}$ away from blue LED lamps $(\lambda=425 \mathrm{~nm})$ in a water bath. The reaction was carried out under visible light irradiation for $8 \mathrm{~h}$ at room temperature and monitored by NMR spectroscopy.

$\left({ }^{1} \mathrm{H}\right.$ NMR)<smiles>C=Cc1ccc(-c2ccccc2)cc1</smiles><smiles></smiles><smiles>[O]C(CCF)c1ccc(-c2ccccc2)cc1</smiles>

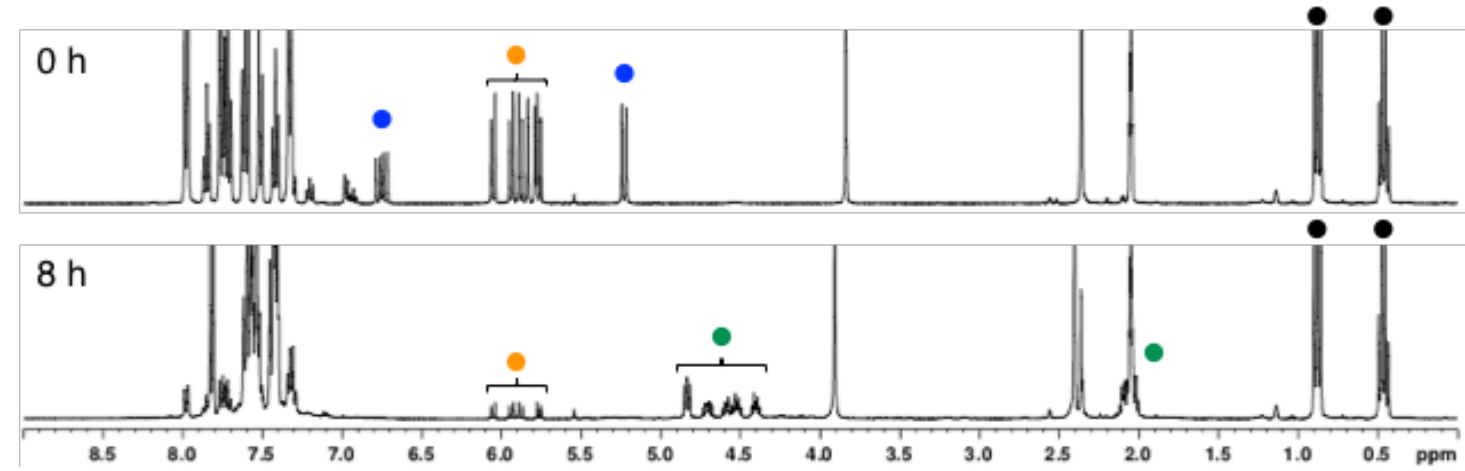

Figure S2. ${ }^{1} \mathrm{H}$ NMR spectra for the reaction of $13 a$ with 3. 
NMR experiment with $\mathbf{5}$<smiles>C=Cc1ccc(-c2ccccc2)cc1</smiles>

p-Vinylbiphenyl 13a (4.5 mg, $25.0 \mu \mathrm{mol}), 5$ (13.6 mg, $37.5 \mu \mathrm{mol}), 1$ (0.58 mg, $1.25 \mu \mathrm{mol})$ and tetraethylsilane were added to an NMR tube under $\mathrm{N}_{2}$ atmosphere. Then, the mixed solvent (acetone- $d_{6}$ : $0.45 \mathrm{~mL}, \mathrm{D}_{2} \mathrm{O}: 0.05 \mathrm{~mL}$ ) was added and the mixture was degassed by three freeze-pump-thaw cycles. The NMR tube was placed at $2-3 \mathrm{~cm}$ away from blue LED lamps $(\lambda=425 \mathrm{~nm})$ in a water bath. The reaction was carried out under visible light irradiation for $8 \mathrm{~h}$ at room temperature and monitored by NMR spectroscopy.

$\left({ }^{1} \mathrm{H}\right.$ NMR)<smiles>C=Cc1ccc(-c2ccccc2)cc1</smiles><smiles>Cc1cc([SH](CF)c2ccccc2)c(C)c(C)c1C</smiles><smiles>O=C(CCF)c1ccc(-c2ccccc2)cc1</smiles><smiles>CCSc1ccccc1</smiles>
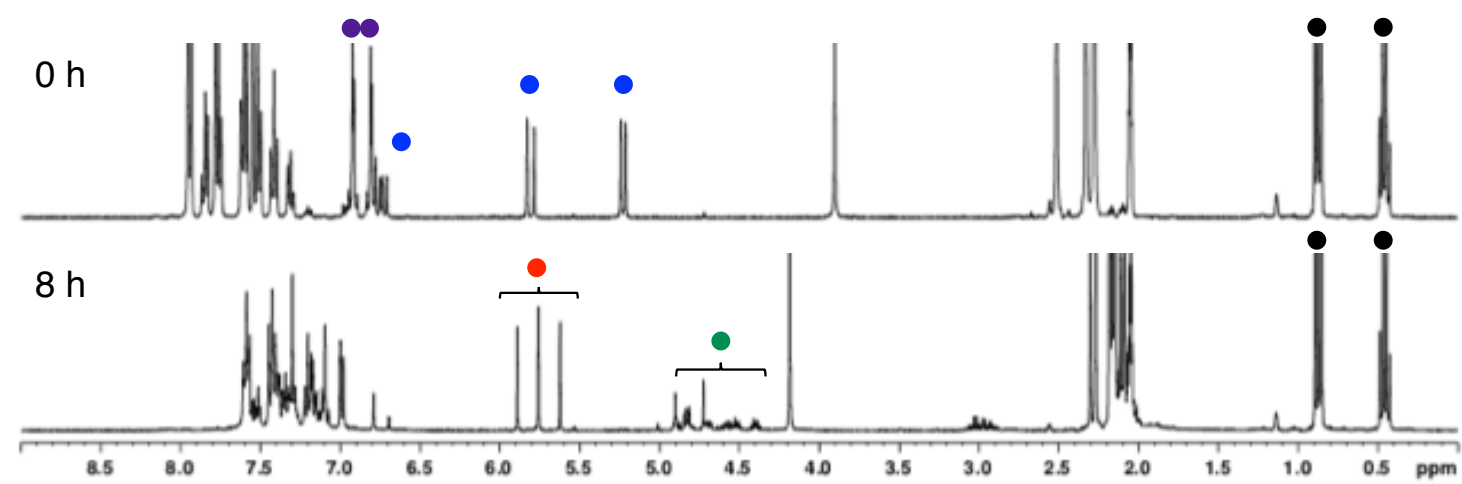

Figure S3. ${ }^{1} \mathrm{H}$ NMR spectra for the reaction of 13a with 5. 
(GC-MS)

Conditions: The products were separated on a $30 \mathrm{~m}$ length by $0.25 \mathrm{~mm}$ id, Rxi- $5 \mathrm{~ms}$ column coated with a $0.25 \mu \mathrm{m}$ film. Helium was employed as the carrier gas, with a constant column flow of $1.22 \mathrm{~mL} / \mathrm{min}$. The injector temperature was held constant at $250^{\circ} \mathrm{C}$. The GC oven temperature program was as follows: 50 ${ }^{\circ} \mathrm{C}$ hold $2 \mathrm{~min}$, ramp $15^{\circ} \mathrm{C} / \mathrm{min}$ to $280^{\circ} \mathrm{C}$, and hold for $30 \mathrm{~min}$.

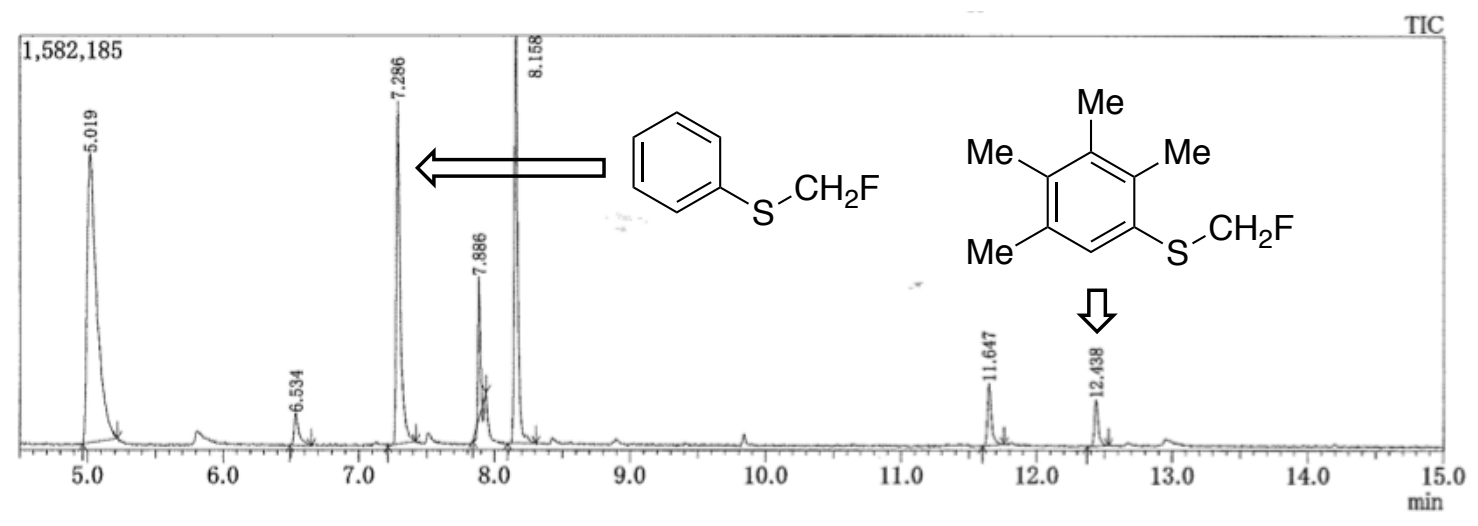<smiles>FCSc1ccccc1</smiles>

$\mathrm{C}_{7} \mathrm{H}_{7} \mathrm{FS}$

Exact Mass: 142.03

(7.286 $\mathrm{min})$

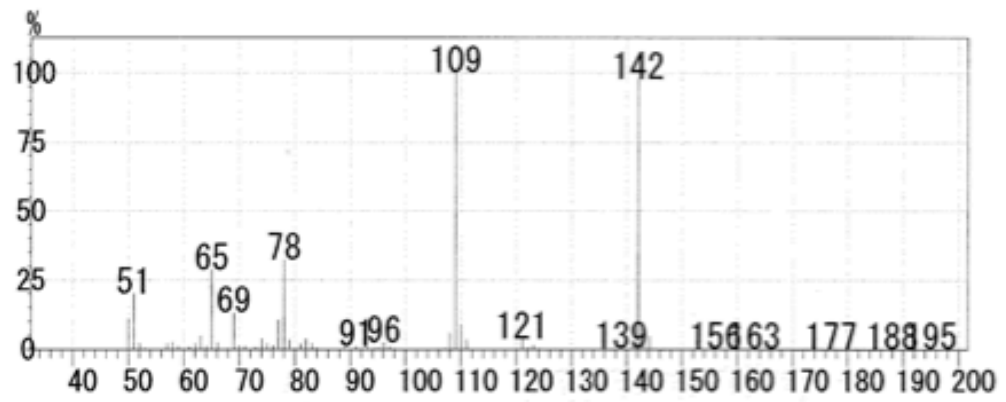<smiles>Cc1cc(SCF)c(C)c(C)c1C</smiles>

Exact Mass: 198.09

(12.438 min)

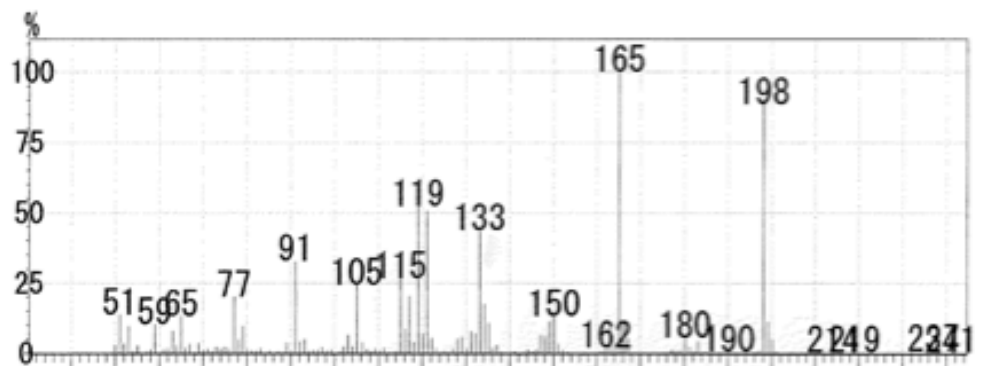

405060708090100110120130140150160170180190200210220230240

Figure S4. A GC chromatogram and MS charts for the reaction of 13a with 5. 
NMR experiment with 3 and 2,2,6,6-tetramethylpiperidine 1-oxyl (TEMPO)

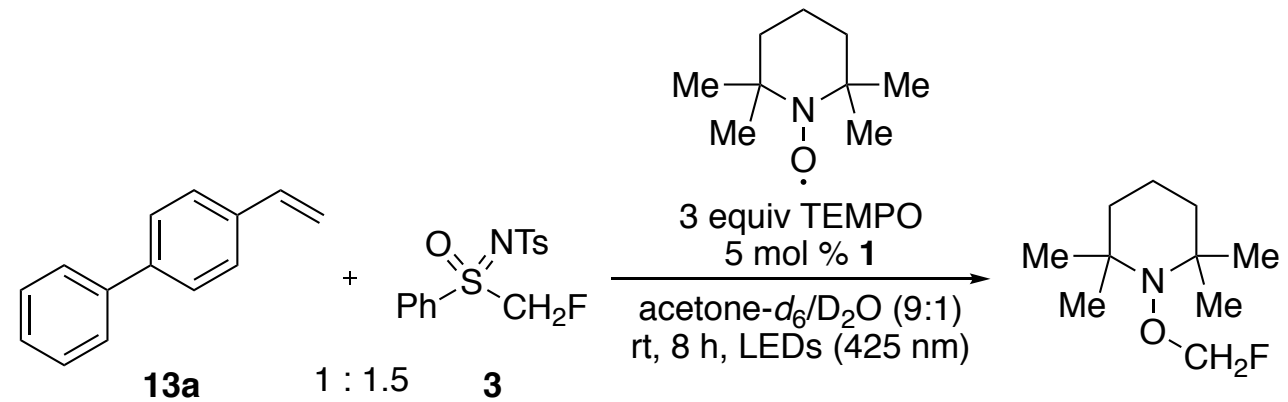

p-Vinylbiphenyl 13a (4.5 mg, $25.0 \mu \mathrm{mol}), 3$ (12.3 mg, $37.6 \mu \mathrm{mol}), 1$ (0.58 mg, $1.25 \mu \mathrm{mol})$, 2,2,6,6tetramethylpiperidine 1-oxyl (TEMPO) $(11.7 \mathrm{mg}, 74.9 \mu \mathrm{mol})$ and tetraethylsilane were added to an NMR tube under $\mathrm{N}_{2}$ atmosphere. Then, the mixed solvent (acetone- $d_{6}: 0.45 \mathrm{~mL}, \mathrm{D}_{2} \mathrm{O}: 0.05 \mathrm{~mL}$ ) was added and the mixture was degassed by three freeze-pump-thaw cycles. The NMR tube was placed at 2-3 cm away from blue LED lamps $(\lambda=425 \mathrm{~nm})$ in a water bath. The reaction was carried out under visible light irradiation for $8 \mathrm{~h}$ at room temperature and monitored by NMR spectroscopy.

$\left({ }^{1} \mathrm{H} N M R\right)$<smiles>C=Cc1ccc(-c2ccccc2)cc1</smiles><smiles>CC[SiH2]C</smiles>

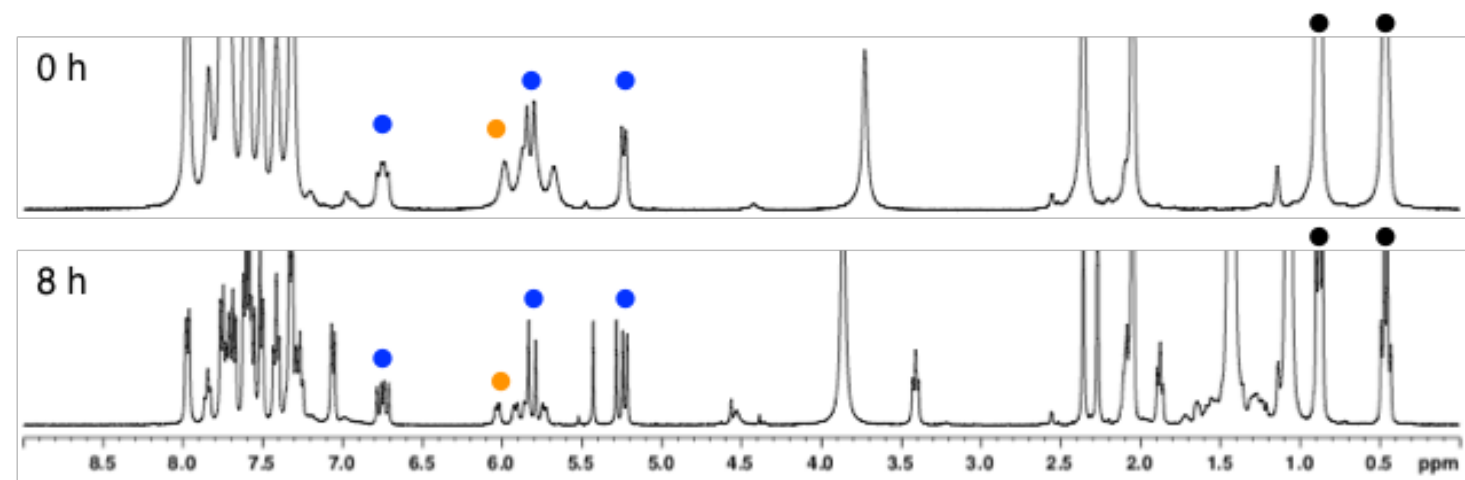

Figure S5. ${ }^{1} \mathrm{H}$ NMR spectra for the reaction of $13 a$ with 3 and TEMPO. 
$\left({ }^{19} \mathrm{~F}\right.$ NMR)
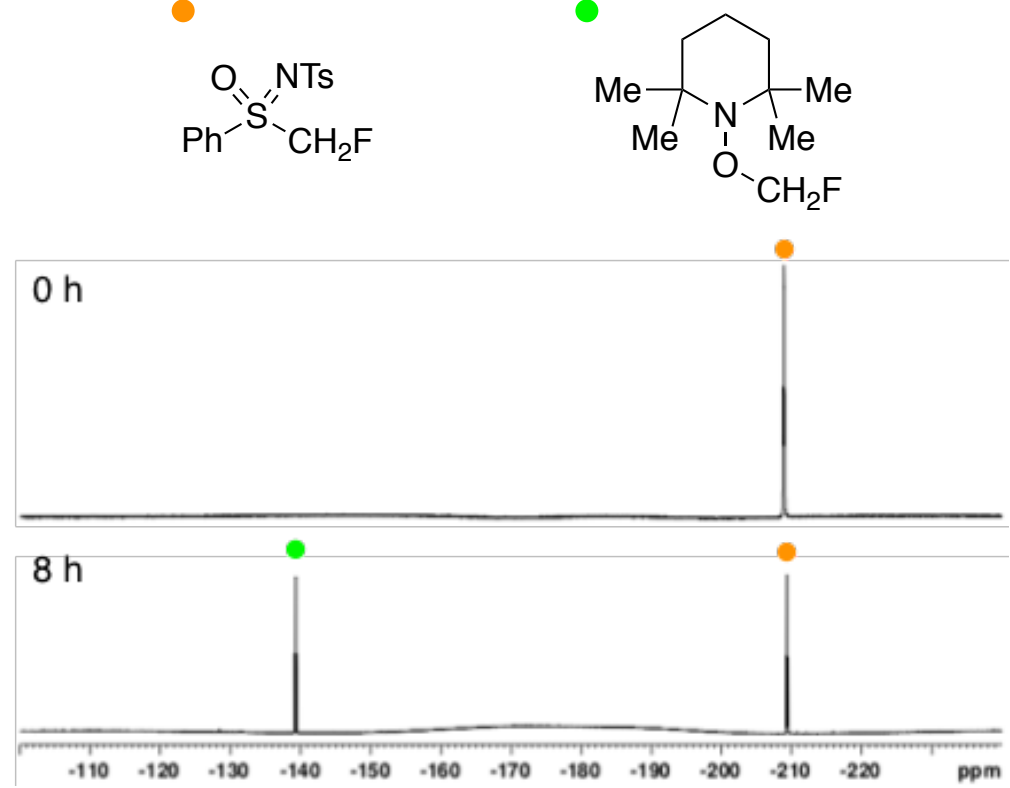

Figure S6. ${ }^{19} \mathrm{~F}$ NMR spectra for the reaction of 13a with $\mathbf{3}$ and TEMPO.

(GC-MS)

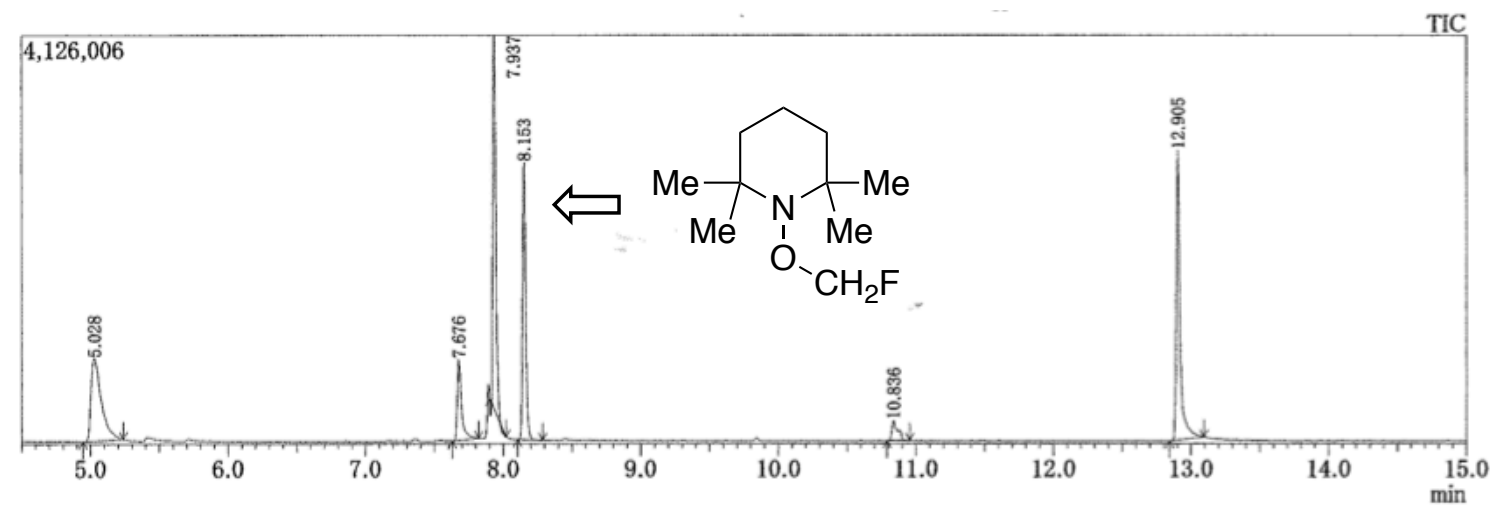

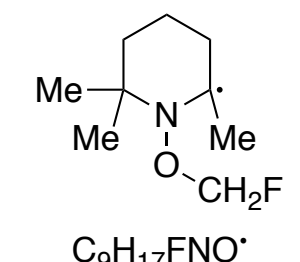

$\mathrm{C}_{9} \mathrm{H}_{17} \mathrm{FNO}^{\circ}$

Exact Mass: 174.13

(8.153 $\mathrm{min})$

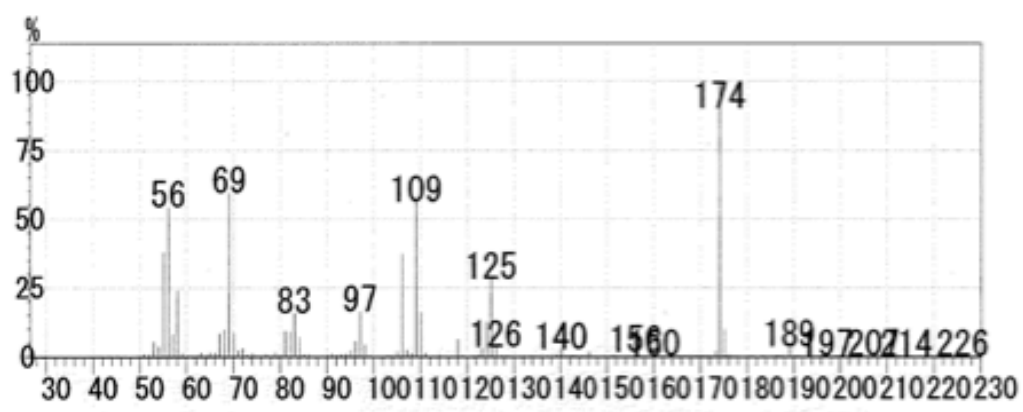

Figure S7. A GC chromatogram and a MS chart for the reaction of 13a with $\mathbf{3}$ and TEMPO. 
General Procedures for Hydroxy-Monofluoromethylation and Characterization

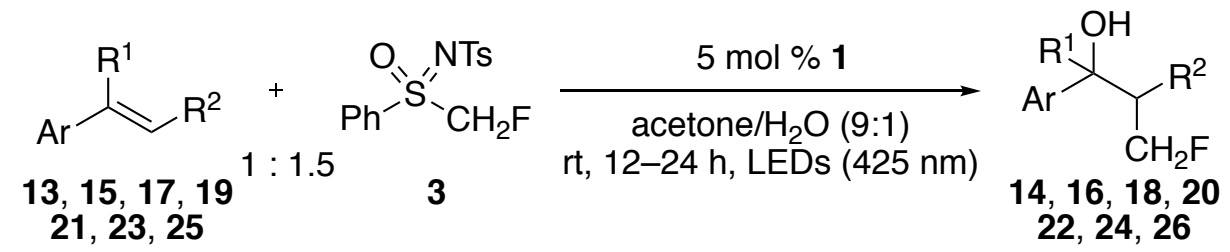

A $20 \mathrm{~mL}$ Schlenk tube containing an aromatic alkene $(0.250 \mathrm{mmol}), 3(0.375 \mathrm{mmol}), 1(12.5 \mu \mathrm{mol})$, and acetone $(4.5 \mathrm{~mL})$ and $\mathrm{H}_{2} \mathrm{O}(0.5 \mathrm{~mL})$ was degassed by three freeze-pump-thaw cycles, and placed at 2-3 $\mathrm{cm}$ away from blue LED lamps $(\lambda=425 \mathrm{~nm})$ in a water bath. The mixture was stirred for $12-24 \mathrm{~h}$ at room temperature under visible light irradiation. After the reaction, the reaction mixture was concentrated under reduced pressure. The desired product was obtained after purification by flash column chromatography on silica-gel and recycling preparative HPLC.

1-([1,1'-Biphenyl]-4-yl)-3-fluoropropan-1-ol (14a)<smiles>OC(CF)c1ccc(-c2ccccc2)cc1</smiles>

According to the general procedures (reaction time $=12 \mathrm{~h}$ ), $p$-vinylbiphenyl 13a $(45.1 \mathrm{mg}, 0.250 \mathrm{mmol}), 3$ (123 mg, $0.375 \mathrm{mmol}), 1(5.8 \mathrm{mg}, 12.5 \mu \mathrm{mol})$, acetone $(4.5 \mathrm{~mL})$ and $\mathrm{H}_{2} \mathrm{O}(0.5 \mathrm{~mL})$ afforded $14 \mathrm{a}$ as a white solid (40.3 mg, $0.175 \mathrm{mmol}, 70 \%)$. Eluent: Hexane/ethyl acetate $=4: 1$.

${ }^{1} \mathrm{H}$ NMR $\left(400 \mathrm{MHz}, \mathrm{CDCl}_{3}\right.$, rt): $\delta$ 7.61-7.58 $(4 \mathrm{H} ; A r), 7.46-7.42(4 \mathrm{H} ; A r), 7.37-7.33(1 \mathrm{H} ; A r), 4.99(\mathrm{~m}, 1 \mathrm{H}$; $\mathrm{CH}_{2} \mathrm{CHOH}$ ), 4.81-4.47 (2H; $\left.\mathrm{CH}_{2} \mathrm{~F}\right), 2.26-2.07\left(2 \mathrm{H} ; \mathrm{CH}_{2} \mathrm{CHOH}\right), 2.02$ (d, J=2.6 Hz, $1 \mathrm{H} ; \mathrm{CH}_{2} \mathrm{CHOH}$ ).

${ }^{13} \mathrm{C}$ NMR $\left(125 \mathrm{MHz}, \mathrm{CDCl}_{3}, \mathrm{rt}\right): \delta 143.1,140.9,140.8,128.9,127.5(2 \mathrm{C}), 127.2,126.3,81.6$ (d, $J=162.3$ $\mathrm{Hz}), 70.8$ (d, $J=4.7 \mathrm{~Hz}), 39.7$ (d, $J=19.0 \mathrm{~Hz})$.

${ }^{19} \mathrm{~F}$ NMR (376 MHz, $\mathrm{CDCl}_{3}$, r.t.): $\delta-222.2(\mathrm{~m}, 1 \mathrm{~F})$.

HRMS (ESI-TOF) calcd m/z for [ $\left.\mathrm{C}_{15} \mathrm{H}_{15} \mathrm{FO}+\mathrm{Na}\right]^{+} 253.0999$ found 253.0999.

Melting point: $69^{\circ} \mathrm{C}$.

3-Fluoro-1-phenylpropan-1-ol (14b)<smiles>OC(CCF)c1ccccc1</smiles>

According to the general procedures (reaction time $=12 \mathrm{~h}$ ), styrene $13 \mathrm{~b}(26.0 \mathrm{mg}, 0.250 \mathrm{mmol}), 3(123$ $\mathrm{mg}, 0.375 \mathrm{mmol}), 1(5.8 \mathrm{mg}, 12.5 \mu \mathrm{mol})$, acetone $(4.5 \mathrm{~mL})$ and $\mathrm{H}_{2} \mathrm{O}(0.5 \mathrm{~mL})$ afforded $14 \mathrm{~b}$ as a colorless oil (20.6 mg, $0.134 \mathrm{mmol}, 54 \%)$. Eluent: Hexane/ethyl acetate $=4: 1$.

${ }^{1} \mathrm{H}$ NMR (400 MHz, CDCl 3 , rt): $\delta$ 7.37-7.28 (5H; $\left.A r\right), 4.91$ (dd, J = 8.4 Hz, $\left.5.0 \mathrm{~Hz}, 1 \mathrm{H} ; \mathrm{CH}_{2} \mathrm{CHOH}\right), 4.76-$ $4.42\left(2 \mathrm{H} ; \mathrm{CH}_{2} \mathrm{~F}\right), 2.22-2.02\left(2 \mathrm{H} ; \mathrm{CH}_{2} \mathrm{CHOH}\right), 2.00$ (brs, $\left.1 \mathrm{H} ; \mathrm{CH}_{2} \mathrm{CHOH}\right)$. 
${ }^{13} \mathrm{C}$ NMR $\left(125 \mathrm{MHz}, \mathrm{CDCl}_{3}, \mathrm{rt}\right): \delta 144.1,128.8,128.0,125.9,81.6(\mathrm{~d}, J=162.2 \mathrm{~Hz}), 71.0(\mathrm{~d}, J=4.5 \mathrm{~Hz})$, $39.7(\mathrm{~d}, \mathrm{~J}=18.9 \mathrm{~Hz})$.

${ }^{19} \mathrm{~F} \mathrm{NMR}\left(376 \mathrm{MHz}, \mathrm{CDCl}_{3}\right.$, r.t.): $\delta-222.3(\mathrm{~m}, 1 \mathrm{~F})$.

HRMS (ESI-TOF) calcd m/z for [ $\left.\mathrm{C}_{9} \mathrm{H}_{11} \mathrm{FO}+\mathrm{Na}\right]^{+} 177.0686$ found 177.0682 .

3-Fluoro-1-(p-tolyl)propan-1-ol (14c)<smiles>Cc1ccc(C(O)CCF)cc1</smiles>

According to the general procedures (reaction time $=12 \mathrm{~h}$ ), 4-methylstyrene $13 \mathrm{c}(29.5 \mathrm{mg}, 0.250 \mathrm{mmol})$, 3 (123 mg, $0.376 \mathrm{mmol}), 1$ (5.8 mg, $12.5 \mu \mathrm{mol})$, acetone $(4.5 \mathrm{~mL})$ and $\mathrm{H}_{2} \mathrm{O}(0.5 \mathrm{~mL})$ afforded $14 \mathrm{c}$ as a colorless oil (23.3 mg, $0.139 \mathrm{mmol}, 55 \%)$. Eluent: Hexane/ethyl acetate $=4: 1$.

${ }^{1} \mathrm{H}$ NMR $\left(400 \mathrm{MHz}, \mathrm{CDCl}_{3}, \mathrm{rt}\right): \delta 7.26(\mathrm{~d}, J=8.0 \mathrm{~Hz}, 2 \mathrm{H} ; A r), 7.18(\mathrm{~d}, J=8.0 \mathrm{~Hz}, 2 \mathrm{H} ; A r), 4.87$ (dd, $J=8.4$ $\left.\mathrm{Hz}, 5.0 \mathrm{~Hz}, 1 \mathrm{H} ; \mathrm{CH}_{2} \mathrm{CHOH}\right), 4.75-4.41\left(2 \mathrm{H} ; \mathrm{CH}_{2} \mathrm{~F}\right), 2.36$ (s, 3H; $\left.\mathrm{Me}\right), 2.21-1.99\left(2 \mathrm{H} ; \mathrm{CH}_{2} \mathrm{CHOH}\right), 1.96$ (brs, $1 \mathrm{H} ; \mathrm{CH}_{2} \mathrm{CHOH}$ ).

${ }^{13} \mathrm{C}$ NMR $\left(125 \mathrm{MHz}, \mathrm{CDCl}_{3}, \mathrm{rt}\right): \delta 141.1,137.7,129.4,125.8,81.6(\mathrm{~d}, J=162.2 \mathrm{~Hz}), 70.8$ (d, $\left.J=4.8 \mathrm{~Hz}\right)$, $39.6(\mathrm{~d}, J=19.0 \mathrm{~Hz}), 21.2$.

${ }^{19} \mathrm{~F}$ NMR (376 MHz, $\mathrm{CDCl}_{3}$, r.t.): $\delta-222.3(\mathrm{~m}, 1 \mathrm{~F})$.

HRMS (ESI-TOF) calcd m/z for [ $\left.\mathrm{C}_{10} \mathrm{H}_{13} \mathrm{FO}+\mathrm{Na}\right]^{+} 191.0843$ found 191.0842.

3-Fluoro-1-(4-fluorophenyl)propan-1-ol (14d)<smiles>OC(CCF)c1ccc(F)cc1</smiles>

According to the general procedures (reaction time $=12 \mathrm{~h}$ ), 4-fluorostyrene $13 \mathrm{~d}(30.5 \mathrm{mg}, 0.250 \mathrm{mmol}), 3$ (123 mg, $0.376 \mathrm{mmol}), 1$ (5.8 mg, $12.5 \mu \mathrm{mol})$, acetone $(4.5 \mathrm{~mL})$ and $\mathrm{H}_{2} \mathrm{O}(0.5 \mathrm{~mL})$ afforded $14 \mathrm{~d}$ as a colorless oil (22.4 mg, $0.130 \mathrm{mmol}, 52 \%)$. Eluent: Hexane/ethyl acetate $=4: 1$.

${ }^{1} \mathrm{H}$ NMR (400 MHz, $\left.\mathrm{CDCl}_{3}, \mathrm{rt}\right): \delta$ 7.37-7.33 (2H; $\left.\mathrm{Ar}\right), 7.08-7.02(2 \mathrm{H} ; \mathrm{Ar}), 4.93\left(\mathrm{~m}, 1 \mathrm{H} ; \mathrm{CH}_{2} \mathrm{CHOH}\right), 4.77-$ $4.41\left(2 \mathrm{H} ; \mathrm{CH}_{2} \mathrm{~F}\right), 2.21-1.98\left(2 \mathrm{H} ; \mathrm{CH}_{2} \mathrm{CHOH}\right), 2.00$ (brs, $\left.1 \mathrm{H} ; \mathrm{CH}_{2} \mathrm{CHOH}\right)$.

${ }^{13} \mathrm{C}$ NMR $\left(125 \mathrm{MHz} \mathrm{CDCl}_{3}, \mathrm{rt}\right): \delta 162.4$ (d, $\left.J=244.3 \mathrm{~Hz}\right), 139.8$ (d, $\left.J=9.7 \mathrm{~Hz}\right), 127.5$ (d, $\left.J=8.0 \mathrm{~Hz}\right), 115.6$ (d, $J=21.3 \mathrm{~Hz}$ ), 81.5 (d, $J=162.4 \mathrm{~Hz}), 70.4$ (d, $J=4.3 \mathrm{~Hz}), 39.8$ (d, $J=18.9 \mathrm{~Hz}$ ).

${ }^{19} \mathrm{~F}$ NMR (376 MHz, $\mathrm{CDCl}_{3}$, r.t.): $\delta-115.7$ (m, 1F; $\left.\mathrm{Ar} F\right),-222.4\left(\mathrm{~m}, 1 \mathrm{~F} ; \mathrm{CH}_{2} F\right)$.

HRMS (ESI-TOF) calcd $\mathrm{m} / \mathrm{z}$ for $\left[\mathrm{C}_{9} \mathrm{H}_{10} \mathrm{~F}_{2} \mathrm{O}+\mathrm{Na}\right]^{+} 195.0592$ found 195.0588 . 
1-(4-Chlorophenyl)-3-fluoropropan-1-ol (14e)<smiles>OC(CCF)c1ccc(Cl)cc1</smiles>

According to the general procedures (reaction time $=12 \mathrm{~h}$ ), 4-chlorostyrene $13 \mathrm{e}(34.6 \mathrm{mg}, 0.250 \mathrm{mmol}$ ), 3 (123 mg, $0.375 \mathrm{mmol}), 1(5.8 \mathrm{mg}, 12.5 \mu \mathrm{mol})$, acetone $(4.5 \mathrm{~mL})$ and $\mathrm{H}_{2} \mathrm{O}(0.5 \mathrm{~mL})$ afforded $14 \mathrm{e}$ as a colorless oil (24.2 mg, $0.128 \mathrm{mmol}, 51 \%)$. Eluent: Hexane/ethyl acetate $=4: 1$.

${ }^{1} \mathrm{H}$ NMR (400 MHz, CDCl 3 , rt): $\delta$ 7.35-7.30 (4H; Ar), $4.92\left(\mathrm{~m}, 1 \mathrm{H} ; \mathrm{CH}_{2} \mathrm{CHOH}\right), 4.77-4.42\left(2 \mathrm{H} ; \mathrm{CH}_{2} \mathrm{~F}\right)$, 2.19-1.99 (2H; $\left.\mathrm{CH}_{2} \mathrm{CHOH}\right), 2.02$ (brs, $\left.1 \mathrm{H} ; \mathrm{CH}_{2} \mathrm{CHOH}\right)$.

${ }^{13} \mathrm{C}$ NMR $\left(125 \mathrm{MHz}, \mathrm{CDCl}_{3}, \mathrm{rt}\right): \delta$ 142.6, 133.6, 128.9, 127.3, 81.4 (d, J = 162.4 Hz), 70.4 (d, J = 4.2 Hz), $39.7(\mathrm{~d}, \mathrm{~J}=18.9 \mathrm{~Hz})$.

${ }^{19} \mathrm{~F}$ NMR (376 MHz, $\mathrm{CDCl}_{3}$, r.t.): $\delta-222.4$ (m, 1F; $\left.\mathrm{CH}_{2} F\right)$.

HRMS (ESI-TOF) calcd m/z for [ $\left.\mathrm{C}_{9} \mathrm{H}_{10} \mathrm{ClFO}+\mathrm{Na}\right]^{+} 211.0296$ found 211.0294.

1-(4-Bromophenyl)-3-fluoropropan-1-ol (14f)<smiles>OC(CCF)c1ccc(Br)cc1</smiles>

According to the general procedures (reaction time $=12 \mathrm{~h}$ ), 4-bromostyrene $13 \mathrm{f}(45.8 \mathrm{mg}, 0.250 \mathrm{mmol}), 3$ (123 mg, $0.377 \mathrm{mmol}), 1(5.8 \mathrm{mg}, 12.5 \mu \mathrm{mol})$, acetone $(4.5 \mathrm{~mL})$ and $\mathrm{H}_{2} \mathrm{O}(0.5 \mathrm{~mL})$ afforded $14 \mathrm{f}$ as a colorless oil (32.3 mg, $0.139 \mathrm{mmol}, 55 \%)$. Eluent: Hexane/ethyl acetate $=4: 1$.

${ }^{1} \mathrm{H}$ NMR (400 MHz, $\left.\mathrm{CDCl}_{3}, \mathrm{rt}\right): \delta 7.49(\mathrm{~d}, \mathrm{~J}=8.4 \mathrm{~Hz}, 2 \mathrm{H} ; A r), 7.26(\mathrm{~d}, \mathrm{~J}=8.4 \mathrm{~Hz}, 2 \mathrm{H} ; A r), 4.91(\mathrm{~m}, 1 \mathrm{H}$; $\left.\mathrm{CH}_{2} \mathrm{CHOH}\right), 4.78-4.42\left(2 \mathrm{H} ; \mathrm{CH}_{2} \mathrm{~F}\right), 2.18-1.97\left(2 \mathrm{H} ; \mathrm{CH}_{2} \mathrm{CHOH}\right), 2.03$ (brs, $1 \mathrm{H} ; \mathrm{CH}_{2} \mathrm{CHOH}$ ).

${ }^{13} \mathrm{C}$ NMR $\left(125 \mathrm{MHz}, \mathrm{CDCl}_{3}, \mathrm{rt}\right): \delta 143.1,131.8,127.6,121.7,81.4$ (d, $\left.J=162.5 \mathrm{~Hz}\right), 70.5$ (d, J = 4.2 Hz), $39.7(\mathrm{~d}, J=18.8 \mathrm{~Hz})$.

${ }^{19} \mathrm{~F}$ NMR (376 MHz, $\mathrm{CDCl}_{3}$, r.t.): $\delta-222.4$ (m, 1F; $\left.\mathrm{CH}_{2} \mathrm{~F}\right)$.

HRMS (ESI-TOF) calcd m/z for [ $\left.\mathrm{C}_{9} \mathrm{H}_{10} \mathrm{BrFO}+\mathrm{Na}\right]^{+} 254.9791$ found 254.9791.

3-Fluoro-1-(4-methoxyphenyl)propan-1-ol (14g)<smiles>COc1ccc(C(O)CCF)cc1</smiles>

According to the general procedures (reaction time $=12 \mathrm{~h}$ ), 4-methoxystyrene $13 \mathrm{~g}(33.5 \mathrm{mg}, 0.250 \mathrm{mmol}$ ), 3 (123 mg, $0.376 \mathrm{mmol}), 1$ (5.8 mg, $12.5 \mu \mathrm{mol})$, acetone $(4.5 \mathrm{~mL})$ and $\mathrm{H}_{2} \mathrm{O}(0.5 \mathrm{~mL})$ afforded $\mathbf{1 4 g}$ as a colorless oil (31.1 mg, $0.169 \mathrm{mmol}, 68 \%)$. Eluent: Hexane/ethyl acetate $=4: 1$. 
${ }^{1} \mathrm{H}$ NMR $\left(400 \mathrm{MHz}, \mathrm{CDCl}_{3}, \mathrm{rt}\right): \delta 7.30(\mathrm{~d}, \mathrm{~J}=8.7 \mathrm{~Hz}, 2 \mathrm{H} ; A r), 6.91(\mathrm{~d}, \mathrm{~J}=8.7 \mathrm{~Hz}, 2 \mathrm{H} ; A r), 4.88(\mathrm{~m}, 1 \mathrm{H}$; $\left.\mathrm{CH}_{2} \mathrm{CHOH}\right), 4.75-4.41\left(2 \mathrm{H} ; \mathrm{CH}_{2} \mathrm{~F}\right), 3.81$ (s, 3H; OMe), 2.24-1.98 (2H; $\left.\mathrm{CH}_{2} \mathrm{CHOH}\right), 1.90$ (brs, $1 \mathrm{H}$; $\left.\mathrm{CH}_{2} \mathrm{CHOH}\right)$.

${ }^{13} \mathrm{C}$ NMR $\left(125 \mathrm{MHz}, \mathrm{CDCl}_{3}, \mathrm{rt}\right): \delta$ 159.4, 136.2, 127.2, 114.1, 81.6 (d, $\left.J=162.2 \mathrm{~Hz}\right), 70.6(\mathrm{~d}, J=4.8 \mathrm{~Hz})$, $55.4,39.6(\mathrm{~d}, J=19.0 \mathrm{~Hz})$.

${ }^{19} \mathrm{~F}$ NMR $\left(376 \mathrm{MHz}, \mathrm{CDCl}_{3}\right.$, r.t.): $\delta-222.4\left(\mathrm{~m}, 1 \mathrm{~F} ; \mathrm{CH}_{2} F\right)$.

HRMS (ESI-TOF) calcd m/z for $\left[\mathrm{C}_{10} \mathrm{H}_{13} \mathrm{FO}_{2}+\mathrm{Na}\right]^{+} 207.0792$ found 207.0792 .

4-(3-Fluoro-1-hydroxypropyl)phenyl acetate (14h)<smiles>CC(=O)Oc1ccc(C(O)CCF)cc1</smiles>

According to the general procedures (reaction time $=12 \mathrm{~h}$ ), 4-acetoxystyrene $13 \mathrm{~h}(40.5 \mathrm{mg}, 0.250 \mathrm{mmol})$, 3 (123 mg, $0.375 \mathrm{mmol}), 1$ (5.8 mg, $12.5 \mu \mathrm{mol})$, acetone $(4.5 \mathrm{~mL})$ and $\mathrm{H}_{2} \mathrm{O}(0.5 \mathrm{~mL})$ afforded $14 \mathrm{~h}$ as a colorless oil (31.5 mg, $0.148 \mathrm{mmol}, 59 \%)$. Eluent: Hexane/ethyl acetate $=4: 1$.

${ }^{1} \mathrm{H}$ NMR (400 MHz, CDCl 3, rt): $\delta 7.39$ (d, J = 8.5 Hz, 2H; Ar), 7.09 (d, J = 8.5 Hz, 2H; Ar), 4.94 (dd, J = 8.3 $\left.\mathrm{Hz}, 4.8 \mathrm{~Hz}, 1 \mathrm{H} ; \mathrm{CH}_{2} \mathrm{CHOH}\right), 4.78-4.43\left(2 \mathrm{H} ; \mathrm{CH}_{2} \mathrm{~F}\right), 2.30$ (s, 3H; OAc), 2.21-2.01 (2H; $\left.\mathrm{CH}_{2} \mathrm{CHOH}\right), 2.00$ (brs, $1 \mathrm{H} ; \mathrm{CH}_{2} \mathrm{CHOH}$ ).

${ }^{13} \mathrm{C}$ NMR $\left(125 \mathrm{MHz}, \mathrm{CDCl}_{3}, \mathrm{rt}\right): \delta 169.7,150.2,141.7,127.0,121.8,81.5(\mathrm{~d}, J=162.4 \mathrm{~Hz}), 70.4(\mathrm{~d}, J=$ $3.8 \mathrm{~Hz}), 39.6(\mathrm{~d}, J=19.0 \mathrm{~Hz}), 21.2$.

${ }^{19} \mathrm{~F}$ NMR (376 $\mathrm{MHz}, \mathrm{CDCl}_{3}$, r.t.): $\delta-222.4\left(\mathrm{~m}, 1 \mathrm{~F} ; \mathrm{CH}_{2} \mathrm{~F}\right)$.

HRMS (ESI-TOF) calcd $\mathrm{m} / \mathrm{z}$ for $\left[\mathrm{C}_{11} \mathrm{H}_{13} \mathrm{FO}_{3}+\mathrm{Na}\right]^{+} 235.0741$ found 235.0739 .

3-Fluoro-1-(4-(trimethylsilyl)phenyl)propan-1-ol (14i)<smiles>C[Si](C)(C)c1ccc(C(O)CCF)cc1</smiles>

According to the general procedures (reaction time $=12 \mathrm{~h}$ ), trimethyl(4-vinylphenyl)silane $13 \mathbf{i}(44.1 \mathrm{mg}$, $0.250 \mathrm{mmol}$ ), 3 (123 mg, $0.377 \mathrm{mmol}), 1(5.8 \mathrm{mg}, 12.5 \mu \mathrm{mol})$, acetone $(4.5 \mathrm{~mL})$ and $\mathrm{H}_{2} \mathrm{O}(0.5 \mathrm{~mL})$ afforded $14 \mathbf{i}$ as a colorless oil $(30.9 \mathrm{mg}, 0.137 \mathrm{mmol}, 55 \%)$. Eluent: Hexane/ethyl acetate $=4: 1$.

${ }^{1} \mathrm{H}$ NMR (400 MHz, $\left.\mathrm{CDCl}_{3}, \mathrm{rt}\right): \delta 7.54(\mathrm{~d}, J=7.9 \mathrm{~Hz}, 2 \mathrm{H} ; A r), 7.36$ (d, J = 7.9 Hz, 2H; Ar), 4.91 (dd, J=8.2 $\left.\mathrm{Hz}, 5.0 \mathrm{~Hz}, 1 \mathrm{H} ; \mathrm{CH}_{2} \mathrm{CHOH}\right), 4.78-4.43\left(2 \mathrm{H} ; \mathrm{CH}_{2} \mathrm{~F}\right), 2.22-2.02\left(2 \mathrm{H} ; \mathrm{CH}_{2} \mathrm{CHOH}\right), 1.98$ (brs, $\left.1 \mathrm{H} ; \mathrm{CH}_{2} \mathrm{CHOH}\right)$, 0.28 (s, 9H; $\left.\mathrm{SiMe}_{3}\right)$.

${ }^{13} \mathrm{C}$ NMR $\left(125 \mathrm{MHz}, \mathrm{CDCl}_{3}, \mathrm{rt}\right): \delta 144.6,140.3,133.8,125.3,81.6$ (d, $\left.J=162.3 \mathrm{~Hz}\right), 70.9$ (d, $\left.J=4.5 \mathrm{~Hz}\right)$, $39.6(\mathrm{~d}, J=19.0 \mathrm{~Hz}),-1.00$.

${ }^{19} \mathrm{~F}$ NMR (376 MHz, $\mathrm{CDCl}_{3}$, r.t.): $\delta-222.3$ (m, $\left.1 \mathrm{~F} ; \mathrm{CH}_{2} \mathrm{~F}\right)$. 
HRMS (ESI-TOF) calcd m/z for [ $\left.\mathrm{C}_{12} \mathrm{H}_{19} \mathrm{FOSi}+\mathrm{Na}\right]^{+} 249.1081$ found 249.1080.

2-(Fluoromethyl)-2,3-dihydro-1H-inden-1-ol (14j)<smiles>OC1c2ccccc2CC1CF</smiles>

14j

According to the general procedures (reaction time $=24 \mathrm{~h}$ ), indene 13j $(29.0 \mathrm{mg}, 0.250 \mathrm{mmol}), 3(123 \mathrm{mg}$, $0.377 \mathrm{mmol}), 1(9.3 \mathrm{mg}, 20.1 \mu \mathrm{mol})$, acetone $(4.5 \mathrm{~mL})$ and $\mathrm{H}_{2} \mathrm{O}(0.5 \mathrm{~mL})$ afforded $14 \mathrm{j}$ as a colorless oil $(11.0 \mathrm{mg}, 0.0662 \mathrm{mmol}, 27 \%$, diastereomeric ratio = 1.1:1). Eluent: Hexane/ethyl acetate $=4: 1$.

${ }^{1} \mathrm{H}$ NMR $\left(400 \mathrm{MHz}, \mathrm{CDCl}_{3}, \mathrm{rt}\right): \delta 7.44-7.22(2 \mathrm{H}$; $A r$ of major and minor isomers), $5.26(\mathrm{~d}, \mathrm{~J}=6.0 \mathrm{~Hz}, 1 \mathrm{H}$; $\mathrm{CHCHOH}$ of minor isomer), $5.26\left(\mathrm{~d}, J=6.4 \mathrm{~Hz}, 1 \mathrm{H}\right.$; $\mathrm{CHCHOH}$ of major isomer), $4.92-4.53\left(2 \mathrm{H} ; \mathrm{CH}_{2} \mathrm{~F}\right.$ of major and minor isomers), 3.16-2.57 (3H; $\mathrm{CHCHOH}, \mathrm{ArCH}_{2} \mathrm{CH}$ of major and minor isomers), 1.78 (brs, $1 \mathrm{H} ; \mathrm{CHCHOH}$ of major and minor isomers).

${ }^{13} \mathrm{C}$ NMR (125 MHz, CDCl $\left.3, \mathrm{rt}\right): \delta 84.6(\mathrm{~d}, J=166.8 \mathrm{~Hz}), 83.7(\mathrm{~d}, J=163.2 \mathrm{~Hz}), 77.8(\mathrm{~d}, J=6.0 \mathrm{~Hz}), 75.8$ (d, $J=4.8 \mathrm{~Hz}$ ), 50.8 (d, $J=18.1 \mathrm{~Hz}), 44.4$ (d, $J=18.3 \mathrm{~Hz}), 32.5$ (d, $J=8.3 \mathrm{~Hz}), 32.1$ (d, $J=6.1 \mathrm{~Hz})$.

Aromatic signals of major and minor diastereomers were overlapped around (144.1, 143.9, 142.4, 141.1, $129.1,128.7,127.2,125.2,125.1,125.0,124.3)$.

${ }^{19} \mathrm{~F}$ NMR (376 MHz, $\mathrm{CDCl}_{3}$, r.t.): $\delta-222.9$ (m, $1 \mathrm{~F} ; \mathrm{CH}_{2} \mathrm{~F}$ of minor isomer), $-223.3\left(\mathrm{~m}, 1 \mathrm{~F} ; \mathrm{CH}_{2} \mathrm{~F}\right.$ of major isomer).

HRMS (ESI-TOF) calcd m/z for [ $\left.\mathrm{C}_{10} \mathrm{H}_{11} \mathrm{FO}+\mathrm{Na}\right]^{+} 189.0686$ found 189.0688.

3-Fluoro-1-(m-tolyl)propan-1-ol (14k)<smiles>Cc1cccc(C(O)CCF)c1</smiles>

According to the general procedures (reaction time $=12 \mathrm{~h}), 3$-methylstyrene $13 \mathrm{k}(29.5 \mathrm{mg}, 0.250 \mathrm{mmol})$, 3 (123 mg, $0.376 \mathrm{mmol}), 1(5.8 \mathrm{mg}, 12.5 \mu \mathrm{mol})$, acetone $(4.5 \mathrm{~mL})$ and $\mathrm{H}_{2} \mathrm{O}(0.5 \mathrm{~mL})$ afforded $14 \mathrm{k}$ as a colorless oil (19.7mg, $0.117 \mathrm{mmol}, 47 \%)$. Eluent: Hexane/ethyl acetate $=4: 1$.

${ }^{1} \mathrm{H}$ NMR (400 MHz, CDCl 3 , rt): $\delta$ 7.28-7.11 (4H; $\left.\mathrm{Ar}\right), 4.89\left(\mathrm{~m}, 1 \mathrm{H} ; \mathrm{CH}_{2} \mathrm{CHOH}\right), 4.77-4.43\left(2 \mathrm{H} ; \mathrm{CH}_{2} \mathrm{~F}\right), 2.37$ (s, 3H; $\mathrm{Me}$ ), 2.23-2.02 (2H; $\left.\mathrm{CH}_{2} \mathrm{CHOH}\right), 1.96$ (brd, $\mathrm{J}=2.7 \mathrm{~Hz}, 1 \mathrm{H} ; \mathrm{CH}_{2} \mathrm{CHOH}$ ).

${ }^{13} \mathrm{C}$ NMR $\left(125 \mathrm{MHz}, \mathrm{CDCl}_{3}\right.$, rt): $\delta$ 144.1, 138.5, 128.70, 128.67, 126.6, 122.9, 81.6 (d, $\left.J=162.3 \mathrm{~Hz}\right), 71.0$ (d, $J=4.4 \mathrm{~Hz}), 39.7$ (d, $J=19.0 \mathrm{~Hz}), 21.6$.

${ }^{19} \mathrm{~F}$ NMR (376 MHz, $\mathrm{CDCl}_{3}$, r.t.): $\delta-222.3(\mathrm{~m}, 1 \mathrm{~F})$.

HRMS (ESI-TOF) calcd $\mathrm{m} / \mathrm{z}$ for $\left[\mathrm{C}_{10} \mathrm{H}_{13} \mathrm{FO}+\mathrm{Na}\right]^{+} 191.0843$ found 191.0848. 
3-Fluoro-1-(o-tolyl)propan-1-ol (14I)<smiles>Cc1ccccc1C(O)CCF</smiles>

141

According to the general procedures (reaction time $=12 \mathrm{~h}$ ), 2-methylstyrene $13 \mathrm{l}(29.5 \mathrm{mg}, 0.250 \mathrm{mmol}), 3$ (123 mg, $0.376 \mathrm{mmol}), 1(5.8 \mathrm{mg}, 12.5 \mu \mathrm{mol})$, acetone $(4.5 \mathrm{~mL})$ and $\mathrm{H}_{2} \mathrm{O}(0.5 \mathrm{~mL})$ afforded $14 \mathrm{l}$ as a colorless oil (21.6 mg, $0.128 \mathrm{mmol}, 51 \%)$. Eluent: Hexane/ethyl acetate $=4: 1$.

${ }^{1} \mathrm{H}$ NMR (400 MHz, $\left.\mathrm{CDCl}_{3}, \mathrm{rt}\right): \delta 7.49$ (d, J = $\left.7.4 \mathrm{~Hz}, 2 \mathrm{H} ; A r\right), 7.27-7.15$ (3H; Ar), 5.17 (dd, J = 8.4 Hz, 5.5 $\left.\mathrm{Hz}, 1 \mathrm{H} ; \mathrm{CH}_{2} \mathrm{CHOH}\right), 4.82-4.46\left(2 \mathrm{H} ; \mathrm{CH}_{2} \mathrm{~F}\right), 2.35$ (s, 3H; $\left.\mathrm{Me}\right), 2.16-1.96\left(2 \mathrm{H} ; \mathrm{CH}_{2} \mathrm{CHOH}\right), 2.00$ (brs, $1 \mathrm{H}$; $\mathrm{CH}_{2} \mathrm{CHOH}$ ).

${ }^{13} \mathrm{C}$ NMR $\left(125 \mathrm{MHz}, \mathrm{CDCl}_{3}, \mathrm{rt}\right): \delta 142.2,134.5,130.6,127.6,126.6,125.1,81.7$ (d, J = 162.2 Hz), 67.2 (d, $J=4.3 \mathrm{~Hz}), 38.7(\mathrm{~d}, J=19.1 \mathrm{~Hz}), 19.0$.

${ }^{19} \mathrm{~F} \mathrm{NMR}\left(376 \mathrm{MHz}, \mathrm{CDCl}_{3}\right.$, r.t.): $\delta-222.0(\mathrm{~m}, 1 \mathrm{~F})$.

HRMS (ESI-TOF) calcd m/z for [ $\left.\mathrm{C}_{10} \mathrm{H}_{13} \mathrm{FO}+\mathrm{Na}\right]^{+} 191.0843$ found 191.0840 .

3-Fluoro-1,1-diphenylpropan-1-ol (16a)<smiles>OC(CCF)(c1ccccc1)c1ccccc1</smiles>

According to the general procedures (reaction time $=12 \mathrm{~h}$ ), 1,1-diphenylethylene $15 \mathrm{a}(45.1 \mathrm{mg}, 0.250$ mmol), 3 (123 mg, $0.375 \mathrm{mmol}), 1$ (5.8 mg, $12.5 \mu \mathrm{mol}$ ), acetone (4.5 mL) and $\mathrm{H}_{2} \mathrm{O}(0.5 \mathrm{~mL})$ afforded 16a as a white oily solid $(48.2 \mathrm{mg}, 0.209 \mathrm{mmol}, 84 \%)$. Eluent: Hexane/ethyl acetate $=10: 1$.

${ }^{1} \mathrm{H}$ NMR $\left(400 \mathrm{MHz}, \mathrm{CDCl}_{3}, \mathrm{rt}\right): \delta 7.43-7.41(4 \mathrm{H} ; A r), 7.35-7.31(4 \mathrm{H} ; A r), 7.26-7.23(2 \mathrm{H} ; A r), 4.56(\mathrm{dt}, J=$ $47.1 \mathrm{~Hz}, 6.4 \mathrm{~Hz}, 2 \mathrm{H} ; \mathrm{CH}_{2} \mathrm{CH}_{2} \mathrm{~F}$ ), 2.77 (dt, J=21.6 Hz, $6.4 \mathrm{~Hz}, 2 \mathrm{H} ; \mathrm{CH}_{2} \mathrm{CH}_{2} \mathrm{~F}$ ), 2.59 (brs, $1 \mathrm{H} ; \mathrm{OH}$ ).

${ }^{13} \mathrm{C}$ NMR $\left(125 \mathrm{MHz}, \mathrm{CDCl}_{3}, \mathrm{rt}\right): \delta 146.3,128.5,127.3,125.9,82.1$ (d, $J=160.0 \mathrm{~Hz}$ ), 77.5 (overlapped by $\left.\mathrm{CDCl}_{3}\right), 41.7$ (d, $\left.J=17.9 \mathrm{~Hz}\right)$.

${ }^{19} \mathrm{~F}$ NMR (376 $\mathrm{MHz}, \mathrm{CDCl}_{3}$, r.t.): $\delta-221.3\left(\mathrm{~m}, 1 \mathrm{~F} ; \mathrm{CH}_{2} \mathrm{~F}\right)$.

HRMS (ESI-TOF) calcd m/z for [ $\left.\mathrm{C}_{15} \mathrm{H}_{15} \mathrm{FO}+\mathrm{Na}\right]^{+} 253.0999$ found 253.1004. 
3-Fluoro-1-phenyl-1-(p-tolyl)propan-1-ol (16b)<smiles>Cc1ccc(C(O)(CCF)c2ccccc2)cc1</smiles>

According to the general procedures (reaction time $=12 \mathrm{~h}), 1$-methyl-4-(1-phenylvinyl)benzene $15 \mathrm{~b}$ (48.6 $\mathrm{mg}, 0.250 \mathrm{mmol}), 3$ (123 mg, $0.375 \mathrm{mmol}), 1$ (5.8 mg, $12.5 \mu \mathrm{mol})$, acetone $(4.5 \mathrm{~mL})$ and $\mathrm{H}_{2} \mathrm{O}(0.5 \mathrm{~mL})$ afforded $16 \mathrm{~b}$ as a colorless oil $(48.2 \mathrm{mg}, 0.197 \mathrm{mmol}, 79 \%)$. Eluent: Hexane/ethyl acetate = 10:1.

${ }^{1} \mathrm{H}$ NMR $\left(400 \mathrm{MHz}, \mathrm{CDCl}_{3}, \mathrm{rt}\right): \delta 7.42-7.40(2 \mathrm{H} ; A r), 7.33-7.28(4 \mathrm{H} ; A r), 7.24(\mathrm{~m}, 1 \mathrm{H} ; A r), 7.14(\mathrm{~d}, J=8.0$ $\mathrm{Hz}, 2 \mathrm{H} ; A r$ ), 4.56 (apparent dtd, $J=47.1 \mathrm{~Hz}, 6.5 \mathrm{~Hz}, 1.2 \mathrm{~Hz}, 2 \mathrm{H} ; \mathrm{CH}_{2} \mathrm{CH}_{2} \mathrm{~F}$ ), 2.74 (apparent dt, $J=21.4$ $\mathrm{Hz}, 6.4 \mathrm{~Hz}, 2 \mathrm{H} ; \mathrm{CH}_{2} \mathrm{CH}_{2} \mathrm{~F}$ ), 2.53 (d, J = 6.0 Hz, 1H; OH), 2.32 (s, 3H, Me).

${ }^{13} \mathrm{C}$ NMR $\left(125 \mathrm{MHz}, \mathrm{CDCl}_{3}, \mathrm{rt}\right): \delta 146.4,143.4,137.0,129.2,128.4,127.2,125.89,125.87,82.2(\mathrm{~d}, \mathrm{~J}=$ $159.9 \mathrm{~Hz}), 77.3(\mathrm{~d}, J=6.6 \mathrm{~Hz}), 41.7(\mathrm{~d}, J=18.1 \mathrm{~Hz}), 21.1$.

${ }^{19} \mathrm{~F}$ NMR (376 MHz, $\mathrm{CDCl}_{3}$, r.t.): $\delta-221.3\left(\mathrm{~m}, 1 \mathrm{~F} ; \mathrm{CH}_{2} F\right)$.

HRMS (ESI-TOF) calcd $\mathrm{m} / \mathrm{z}$ for $\left[\mathrm{C}_{16} \mathrm{H}_{17} \mathrm{FO}+\mathrm{Na}\right]^{+} 267.1156$ found 267.1154 .

3-Fluoro-1-(4-fluorophenyl)-1-phenylpropan-1-ol (16c)<smiles>OC(CCF)(c1ccccc1)c1ccc(F)cc1</smiles>

According to the general procedures (reaction time $=12 \mathrm{~h}$ ), 1-fluoro-4-(1-phenylvinyl)benzene 15c (49.6 $\mathrm{mg}, 0.250 \mathrm{mmol}), 3(123 \mathrm{mg}, 0.376 \mathrm{mmol}), 1(5.8 \mathrm{mg}, 12.5 \mu \mathrm{mol})$, acetone $(4.5 \mathrm{~mL})$ and $\mathrm{H}_{2} \mathrm{O}(0.5 \mathrm{~mL})$ afforded $16 \mathrm{c}$ as a colorless oil (48.1 mg, $0.194 \mathrm{mmol}, 77 \%)$. Eluent: Hexane/ethyl acetate = 10:1.

${ }^{1} \mathrm{H}$ NMR (400 MHz, $\left.\mathrm{CDCl}_{3}, \mathrm{rt}\right): \delta$ 7.41-7.32 (6H; Ar), $7.26(\mathrm{~m}, 1 \mathrm{H} ; \mathrm{Ar}), 7.04-6.98(2 \mathrm{H} ; \mathrm{Ar}), 4.56$ (apparent $\mathrm{dt}, J=47.0 \mathrm{~Hz}, 6.3 \mathrm{~Hz}, 2 \mathrm{H} ; \mathrm{CH}_{2} \mathrm{CH}_{2} \mathrm{~F}$ ), 2.74 (apparent dt, $J=22.2 \mathrm{~Hz}, 6.3 \mathrm{~Hz}, 2 \mathrm{H} ; \mathrm{CH}_{2} \mathrm{CH}_{2} \mathrm{~F}$ ), 2.62 (d, $J$ $=6.8 \mathrm{~Hz}, 1 \mathrm{H} ; \mathrm{OH})$.

${ }^{13} \mathrm{C}$ NMR $\left(125 \mathrm{MHz}, \mathrm{CDCl}_{3}, \mathrm{rt}\right): \delta 161.9$ (d, $\left.J=244.8 \mathrm{~Hz}\right), 146.0,142.1$ (d, $\left.J=2.9 \mathrm{~Hz}\right), 128.6,127.8$ (d, $J$ $=8.2 \mathrm{~Hz}), 127.5,125.9,115.2(\mathrm{~d}, J=21.1 \mathrm{~Hz}), 82.0(\mathrm{~d}, J=160.3 \mathrm{~Hz}), 77.2(\mathrm{~d}, J=6.0 \mathrm{~Hz}), 41.8(\mathrm{~d}, J=$ $17.9 \mathrm{~Hz})$.

${ }^{19} \mathrm{~F} \mathrm{NMR}\left(376 \mathrm{MHz}, \mathrm{CDCl}_{3}\right.$, r.t.): $\delta-116.7(\mathrm{~m}, 1 \mathrm{~F} ; \mathrm{Ar} F),-222.2\left(\mathrm{~m}, 1 \mathrm{~F} ; \mathrm{CH}_{2} F\right)$.

HRMS (ESI-TOF) calcd $\mathrm{m} / \mathrm{z}$ for $\left[\mathrm{C}_{15} \mathrm{H}_{14} \mathrm{~F}_{2} \mathrm{O}+\mathrm{Na}\right]^{+} 271.0905$ found 271.0905. 
1-(4-Chlorophenyl)-3-fluoro-1-phenylpropan-1-ol (16d)<smiles>OC(CCF)(c1ccccc1)c1ccc(Cl)cc1</smiles>

According to the general procedures (reaction time $=12 \mathrm{~h})$, 1-chloro-4-(1-phenylvinyl)benzene $15 \mathrm{~d}(53.7$ $\mathrm{mg}, 0.250 \mathrm{mmol}), 3$ (123 mg, $0.376 \mathrm{mmol}), 1$ (5.8 mg, $12.5 \mu \mathrm{mol})$, acetone $(4.5 \mathrm{~mL})$ and $\mathrm{H}_{2} \mathrm{O}(0.5 \mathrm{~mL})$ afforded $16 \mathrm{~d}$ as a colorless oil $(41.7 \mathrm{mg}, 0.158 \mathrm{mmol}, 63 \%)$. Eluent: Hexane/ethyl acetate = 10:1.

${ }^{1} \mathrm{H}$ NMR (400 MHz, CDCl 3, rt): $\delta$ 7.41-7.24 (9H; $\left.A r\right), 4.56$ (apparent dtd, J = 47.0 Hz, $6.2 \mathrm{~Hz}, 1.7 \mathrm{~Hz}, 2 \mathrm{H}$; $\mathrm{CH}_{2} \mathrm{CH}_{2} \mathrm{~F}$ ), 2.73 (apparent dt, $J=22.3 \mathrm{~Hz}, 6.2 \mathrm{~Hz}, 2 \mathrm{H} ; \mathrm{CH}_{2} \mathrm{CH}_{2} \mathrm{~F}$ ), 2.64 (d, $J=7.0 \mathrm{~Hz}, 1 \mathrm{H} ; \mathrm{OH}$ ).

${ }^{13} \mathrm{C}$ NMR $\left(125 \mathrm{MHz}, \mathrm{CDCl}_{3}, \mathrm{rt}\right): \delta 145.8,144.8,133.1,128.61,128.56,127.6,127.5,125.8,82.0$ (d, $\mathrm{J}=$ $160.1 \mathrm{~Hz}), 77.2$ (d, $J=5.6 \mathrm{~Hz}), 41.6(\mathrm{~d}, J=18.1 \mathrm{~Hz})$.

${ }^{19} \mathrm{~F}$ NMR $\left(376 \mathrm{MHz}, \mathrm{CDCl}_{3}\right.$, r.t.): $\delta-221.2\left(\mathrm{~m}, 1 \mathrm{~F} ; \mathrm{CH}_{2} \mathrm{~F}\right)$.

HRMS (ESI-TOF) calcd $\mathrm{m} / \mathrm{z}$ for $\left[\mathrm{C}_{15} \mathrm{H}_{14} \mathrm{FOCl}+\mathrm{Na}\right]^{+} 287.0609$ found 287.0610 .

1-\{(1,1'-Biphenyl)-4-yl\}-3-fluoro-1-phenylpropan-1-ol (16e)<smiles>OC(CCF)(c1ccccc1)c1ccc(-c2ccccc2)cc1</smiles>

According to the general procedures (reaction time $=12 \mathrm{~h}), 4$-(1-phenylvinyl)-1,1'-biphenyl $15 \mathrm{e}(64.1 \mathrm{mg}$, $0.250 \mathrm{mmol}$ ), 3 (123 mg, $0.376 \mathrm{mmol}), 1(5.8 \mathrm{mg}, 12.5 \mu \mathrm{mol})$, acetone $(4.5 \mathrm{~mL})$ and $\mathrm{H}_{2} \mathrm{O}(0.5 \mathrm{~mL})$ afforded 16e as a colorless oil (59.3 mg, $0.195 \mathrm{mmol}, 77 \%)$. Eluent: Hexane/ethyl acetate $=10: 1$.

${ }^{1} \mathrm{H}$ NMR $\left(400 \mathrm{MHz}, \mathrm{CDCl}_{3}\right.$, rt): $\delta 7.62-7.28\left(14 \mathrm{H} ; A r\right.$ ), 4.62 (apparent dt, J=47.1 Hz, $6.4 \mathrm{~Hz}, 2 \mathrm{H} ; \mathrm{CH}_{2} \mathrm{CH}_{2} \mathrm{~F}$ ), 2.82 (apparent dt, $\mathrm{J}=21.5 \mathrm{~Hz}, 6.4 \mathrm{~Hz}, 2 \mathrm{H} ; \mathrm{CH}_{2} \mathrm{CH}_{2} \mathrm{~F}$ ), 2.73 (brs, $1 \mathrm{H} ; \mathrm{OH}$ ).

${ }^{13} \mathrm{C} \mathrm{NMR}\left(125 \mathrm{MHz}, \mathrm{CDCl}_{3}\right.$, rt): $\delta$ 146.2, 145.3, 140.7, 140.1, 128.9, 128.5, 127.5, 127.3, 127.2 (2C), 126.4, 125.9, 82.2 (d, $J=160.3 \mathrm{~Hz}$ ), 77.4 (overlapped by $\mathrm{CDCl}_{3}$ ), 41.7 (d, $J=18.0 \mathrm{~Hz}$ ).

${ }^{19} \mathrm{~F}$ NMR (376 MHz, $\mathrm{CDCl}_{3}$, r.t.): $\delta-221.1$ (m, $\left.1 \mathrm{~F} ; \mathrm{CH}_{2} \mathrm{~F}\right)$.

HRMS (ESI-TOF) calcd m/z for [ $\left.\mathrm{C}_{21} \mathrm{H}_{19} \mathrm{FO}+\mathrm{Na}\right]^{+} 329.1312$ found 329.1311. 
3-Fluoro-1-(4-methoxyphenyl)-1-phenylpropan-1-ol (16f)<smiles>COc1ccc(C(O)(CCF)c2ccccc2)cc1</smiles>

According to the general procedures (reaction time $=12 \mathrm{~h}), 1$-methoxy-4-(1-phenylvinyl)benzene $15 f(52.6$ $\mathrm{mg}, 0.250 \mathrm{mmol}), 3$ (123 mg, $0.376 \mathrm{mmol}), 1$ (5.8 mg, $12.5 \mu \mathrm{mol})$, acetone $(4.5 \mathrm{~mL})$ and $\mathrm{H}_{2} \mathrm{O}(0.5 \mathrm{~mL})$ afforded $16 \mathrm{f}$ as a colorless oil (42.3 mg, $0.162 \mathrm{mmol}, 65 \%)$. Eluent: Hexane/ethyl acetate = 10:1.

${ }^{1} \mathrm{H}$ NMR $\left(400 \mathrm{MHz}, \mathrm{CDCl}_{3}, \mathrm{rt}\right): \delta$ 7.42-7.39 $(2 \mathrm{H} ; A r), 7.34-7.31(4 \mathrm{H} ; A r), 7.24(\mathrm{~m}, 1 \mathrm{H} ; \mathrm{Ar}), 6.87-6.84(2 \mathrm{H}$; Ar), 4.66-4.45 (2H; $\left.\mathrm{CH}_{2} \mathrm{CH}_{2} \mathrm{~F}\right), 3.79\left(\mathrm{~s}, 3 \mathrm{H}, \mathrm{OMe}\right.$ ), 2.73 (apparent dt, $J=21.6 \mathrm{~Hz}, 6.5 \mathrm{~Hz}, 2 \mathrm{H} ; \mathrm{CH}_{2} \mathrm{CH}_{2} \mathrm{~F}$ ), $2.52(\mathrm{~d}, J=6.0 \mathrm{~Hz}, 1 \mathrm{H} ; \mathrm{OH})$.

${ }^{13} \mathrm{C}$ NMR $\left(125 \mathrm{MHz}, \mathrm{CDCl}_{3}, \mathrm{rt}\right): \delta 158.7,146.4,138.6,128.4,127.23,127.19,125.9,113.7,82.2(\mathrm{~d}, \mathrm{~J}=$ $159.9 \mathrm{~Hz}$ ), 77.2 (overlapped by $\mathrm{CDCl}_{3}$ ), $55.4,41.8(\mathrm{~d}, J=18.1 \mathrm{~Hz}$ ).

${ }^{19} \mathrm{~F}$ NMR (376 MHz, $\mathrm{CDCl}_{3}$, r.t.): $\delta-221.4\left(\mathrm{~m}, 1 \mathrm{~F} ; \mathrm{CH}_{2} \mathrm{~F}\right)$.

HRMS (ESI-TOF) calcd $\mathrm{m} / \mathrm{z}$ for $\left[\mathrm{C}_{16} \mathrm{H}_{17} \mathrm{FO}_{2}+\mathrm{Na}\right]^{+} 283.1105$ found 283.1105.

3-Fluoro-1-(4-nitrophenyl)-1-phenylpropan-1-ol (16g)<smiles>O=[N+]([O-])C1CCCCC1(O)c1ccccc1</smiles>

According to the general procedures (reaction time $=12 \mathrm{~h}$ ), 1-nitro-4-(1-phenylvinyl)benzene $\mathbf{1 5 g}(56.3$ $\mathrm{mg}, 0.250 \mathrm{mmol}), 3$ (123 mg, $0.376 \mathrm{mmol}), 1(5.8 \mathrm{mg}, 12.5 \mu \mathrm{mol})$, acetone $(4.5 \mathrm{~mL})$ and $\mathrm{H}_{2} \mathrm{O}(0.5 \mathrm{~mL})$ afforded $16 \mathrm{~g}$ as a colorless oil $(34.3 \mathrm{mg}, 0.125 \mathrm{mmol}, 50 \%)$. Eluent: Hexane/ethyl acetate $=4: 1$.

${ }^{1} \mathrm{H}$ NMR (400 MHz, CDCl $\left.3, \mathrm{rt}\right): \delta 8.19-8.16(2 \mathrm{H} ; A r), 7.64-7.61(2 \mathrm{H} ; A r), 7.43-7.35(4 \mathrm{H} ; A r), 7.29(\mathrm{~m}, 1 \mathrm{H}$; Ar), 4.71-4.50 (2H; $\left.\mathrm{CH}_{2} \mathrm{CH}_{2} \mathrm{~F}\right), 2.83-2.75\left(2 \mathrm{H} ; \mathrm{CH}_{2} \mathrm{CH}_{2} \mathrm{~F}\right), 2.82$ (brs, $\left.1 \mathrm{H} ; \mathrm{OH}\right)$.

${ }^{13} \mathrm{C}$ NMR $\left(125 \mathrm{MHz}, \mathrm{CDCl}_{3}, \mathrm{rt}\right): \delta 153.4,147.0,145.1,128.9,128.0,126.9,125.8,123.7,81.7$ (d, $J=161.3$ $\mathrm{Hz}$ ), 77.4 (overlapped to $\mathrm{CDCl}_{3}$ ), 41.4 (d, $J=18.0 \mathrm{~Hz}$ ).

${ }^{19} \mathrm{~F}$ NMR (376 MHz, $\mathrm{CDCl}_{3}$, r.t.): $\delta-220.7$ (m, $\left.1 \mathrm{~F} ; \mathrm{CH}_{2} F\right)$.

HRMS (ESI-TOF) calcd m/z for $\left[\mathrm{C}_{15} \mathrm{H}_{14} \mathrm{FO}_{3}+\mathrm{Na}\right]^{+} 298.0850$ found 298.0850. 


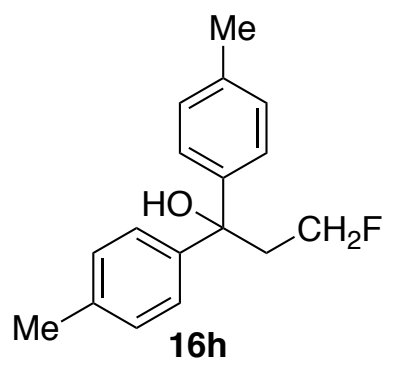

According to the general procedures (reaction time $=12 \mathrm{~h}$ ), 1,1-di-p-tolylethylene $\mathbf{1 5 h}(52.1 \mathrm{mg}, 0.250$ mmol), 3 (123 mg, $0.377 \mathrm{mmol}), 1$ (5.8 mg, $12.5 \mu \mathrm{mol})$, acetone $(4.5 \mathrm{~mL})$ and $\mathrm{H}_{2} \mathrm{O}(0.5 \mathrm{~mL})$ afforded $16 \mathrm{~h}$ as a white oily solid (51.1 mg, $0.209 \mathrm{mmol}, 79 \%)$. Eluent: Hexane/ethyl acetate $=10: 1$.

${ }^{1} \mathrm{H}$ NMR $\left(400 \mathrm{MHz} \mathrm{CDCl}_{3}, \mathrm{rt}\right): \delta 7.28(\mathrm{~d}, J=8.1 \mathrm{~Hz}, 4 \mathrm{H} ; A r), 7.12(\mathrm{~d}, J=8.1 \mathrm{~Hz}, 4 \mathrm{H} ; A r), 4.55$ (dt, J=47.2 $\mathrm{Hz}, 6.5 \mathrm{~Hz}, 2 \mathrm{H} ; \mathrm{CH}_{2} \mathrm{CH}_{2} \mathrm{~F}$ ), $2.72\left(\mathrm{dt}, J=21.2 \mathrm{~Hz}, 6.5 \mathrm{~Hz}, 2 \mathrm{H} ; \mathrm{CH}_{2} \mathrm{CH}_{2} \mathrm{~F}\right), 2.47(\mathrm{~d}, J=5.6 \mathrm{~Hz}, 1 \mathrm{H} ; \mathrm{OH}), 2.32$ (s, 6H; Me).

${ }^{13} \mathrm{C}$ NMR $\left(125 \mathrm{MHz}, \mathrm{CDCl}_{3}, \mathrm{rt}\right): \delta$ 143.6, 136.8, 129.1, 125.8, $82.2(\mathrm{~d}, J=159.9 \mathrm{~Hz}$ ), 77.2 (overlapped by $\left.\mathrm{CDCl}_{3}\right), 41.8(\mathrm{~d}, J=18.2 \mathrm{~Hz}), 21.1$.

${ }^{19} \mathrm{~F}$ NMR (376 $\mathrm{MHz}, \mathrm{CDCl}_{3}$, r.t.): $\delta-221.4\left(\mathrm{~m}, 1 \mathrm{~F} ; \mathrm{CH}_{2} \mathrm{~F}\right)$.

HRMS (ESI-TOF) calcd $\mathrm{m} / \mathrm{z}$ for $\left[\mathrm{C}_{17} \mathrm{H}_{19} \mathrm{FO}+\mathrm{Na}\right]^{+} 281.1312$ found 281.1310.

3-Fluoro-1,1-bis(4-fluorophenyl)propan-1-ol (16i)<smiles>OC(CCF)(c1ccc(F)cc1)c1ccc(F)cc1</smiles>

According to the general procedures (reaction time $=12 \mathrm{~h}$ ), 4,4'-(ethene-1,1-diyl)bis(fluorobenzene) $\mathbf{1 5 i}$ (54.1 mg, $0.250 \mathrm{mmol}), 3$ (123 mg, $0.375 \mathrm{mmol}), 1$ (5.8 mg, $12.5 \mu \mathrm{mol})$, acetone $(4.5 \mathrm{~mL})$ and $\mathrm{H}_{2} \mathrm{O}(0.5$ $\mathrm{mL}$ ) afforded $16 \mathrm{i}$ as a white oily solid $(49.2 \mathrm{mg}, 0.185 \mathrm{mmol}, 74 \%)$. Eluent: Hexane/ethyl acetate $=10: 1$.

${ }^{1} \mathrm{H}$ NMR $\left(400 \mathrm{MHz}, \mathrm{CDCl}_{3}\right.$, rt): $\delta 7.39-7.35(4 \mathrm{H} ; A r), 7.04-6.99(4 \mathrm{H} ; A r), 4.56(\mathrm{dt}, J=47.1 \mathrm{~Hz}, 6.2 \mathrm{~Hz}, 2 \mathrm{H}$; $\mathrm{CH}_{2} \mathrm{CH}_{2} \mathrm{~F}$ ), 2.71 (dt, $J=22.6 \mathrm{~Hz}, 6.2 \mathrm{~Hz}, 2 \mathrm{H} ; \mathrm{CH}_{2} \mathrm{CH}_{2} \mathrm{~F}$ ), 2.64 (d, $J=7.4 \mathrm{~Hz}, 1 \mathrm{H} ; \mathrm{OH}$ ).

${ }^{13} \mathrm{C} \mathrm{NMR}\left(125 \mathrm{MHz}, \mathrm{CDCl}_{3}, \mathrm{rt}\right): \delta 162.0(\mathrm{~d}, J=245.0 \mathrm{~Hz}), 141.9(\mathrm{~d}, J=3.1 \mathrm{~Hz}), 127.7$ (d, J=8.0 Hz), 115.3 (d, $J=21.3 \mathrm{~Hz}$ ), 82.0 (d, $J=160.5 \mathrm{~Hz}$ ), 77.0 (overlapped by $\mathrm{CDCl}_{3}$ ), 41.9 (d, $J=18.0 \mathrm{~Hz}$ ).

${ }^{19} \mathrm{~F}$ NMR (376 MHz, $\mathrm{CDCl}_{3}$, r.t.): $\delta-116.4(\mathrm{~m}, 2 \mathrm{~F} ; \mathrm{ArF}),-221.2\left(\mathrm{~m}, 1 \mathrm{~F} ; \mathrm{CH}_{2} \mathrm{~F}\right)$.

HRMS (ESI-TOF) calcd $\mathrm{m} / \mathrm{z}$ for $\left[\mathrm{C}_{15} \mathrm{H}_{13} \mathrm{~F}_{3} \mathrm{O}+\mathrm{Na}\right]^{+} 289.0811$ found 289.0806 . 
5-(2-Fluoroethyl)-10,11-dihydro-5H-dibenzo[a,d][7]annulen-5-ol (16j)

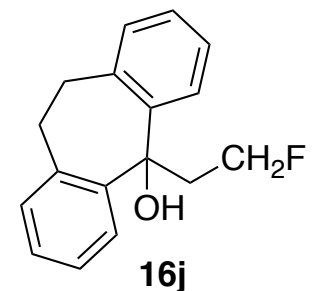

According to the general procedures (reaction time $=12 \mathrm{~h}$ ), 5-methylene-10,11-dihydro- $5 \mathrm{H}$ dibenzo[a,d][7]annulene 15j (51.6 mg, $0.250 \mathrm{mmol}), 3$ (123 mg, $0.377 \mathrm{mmol}), 1$ (5.8 mg, $12.5 \mu \mathrm{mol})$, acetone $(4.5 \mathrm{~mL})$ and $\mathrm{H}_{2} \mathrm{O}(0.5 \mathrm{~mL})$ afforded $16 \mathrm{j}$ as a white oily solid $(37.8 \mathrm{mg}, 0.147 \mathrm{mmol}, 59 \%)$. Eluent: Hexane/ethyl acetate $=10: 1$.

${ }^{1} \mathrm{H}$ NMR (400 MHz, CDCl 3 , rt): $\delta 7.96$ (dd, J=8.0 Hz, $\left.1.2 \mathrm{~Hz}, 2 \mathrm{H} ; A r\right), 7.27$ (ddd, J = 7.6 Hz, 7.6 Hz, 1.4 $\mathrm{Hz}, 2 \mathrm{H} ; A r$ ), 7.21 (dd, J = 7.4 Hz, 7.4 Hz, $1.4 \mathrm{~Hz}, 2 \mathrm{H} ; A r), 7.13$ (d, J = 7.4 Hz, 2H; Ar), 4.35 (dt, J = 47.2 $\left.\mathrm{Hz}, 6.2 \mathrm{~Hz}, 2 \mathrm{H} ; \mathrm{CH}_{2} \mathrm{CH}_{2} \mathrm{~F}\right), 3.40-3.32\left(2 \mathrm{H} ; \mathrm{ArCH}_{2} \mathrm{CH}_{2} \mathrm{Ar}\right), 3.03-2.95\left(2 \mathrm{H} ; \mathrm{ArCH}_{2} \mathrm{CH}_{2} \mathrm{Ar}\right), 2.72$ (d, J = 6.2 $\mathrm{Hz}, 1 \mathrm{H} ; \mathrm{OH}), 2.67$ (dt, $\mathrm{J}=23.6 \mathrm{~Hz}, 6.2 \mathrm{~Hz}, 2 \mathrm{H} ; \mathrm{CH}_{2} \mathrm{CH}_{2} \mathrm{~F}$ ).

${ }^{13} \mathrm{C}$ NMR $\left(125 \mathrm{MHz}, \mathrm{CDCl}_{3}, \mathrm{rt}\right): \delta 143.7,138.6,130.5,127.7,127.0,126.6,81.8(\mathrm{~d}, \mathrm{~J}=160.5 \mathrm{~Hz}), 78.1$ (d, $J=5.2 \mathrm{~Hz}), 44.9(\mathrm{~d}, J=17.9 \mathrm{~Hz}), 34.6$.

${ }^{19} \mathrm{~F}$ NMR $\left(376 \mathrm{MHz}, \mathrm{CDCl}_{3}\right.$, r.t.): $\delta-220.3\left(\mathrm{~m}, 1 \mathrm{~F} ; \mathrm{CH}_{2} F\right)$.

HRMS (ESI-TOF) calcd $\mathrm{m} / \mathrm{z}$ for $\left[\mathrm{C}_{17} \mathrm{H}_{17} \mathrm{FO}+\mathrm{Na}\right]^{+} 279.1156$ found 279.1151.

3-Fluoro-1-phenyl-1-(pyridin-3-yl)propan-1-ol (16k)<smiles>OC(CCF)(c1ccccc1)c1cccnc1</smiles>

According to the general procedures (reaction time $=24 \mathrm{~h})$, 3-(1-phenylvinyl)pyridine 15k (52.6 mg, 0.250 mmol), 3 (123 mg, $0.375 \mathrm{mmol}), 1$ (5.8 mg, $12.5 \mu \mathrm{mol})$, acetone $(4.5 \mathrm{~mL})$ and $\mathrm{H}_{2} \mathrm{O}(0.5 \mathrm{~mL})$ afforded 16k as a colorless oil $(24.1 \mathrm{mg}, 0.104 \mathrm{mmol}, 42 \%)$. Eluent: Hexane/ethyl acetate $=1: 1$.

${ }^{1} \mathrm{H}$ NMR $\left(400 \mathrm{MHz}, \mathrm{CDCl}_{3}, \mathrm{rt}\right): \delta 8.67(\mathrm{~d}, J=1.8 \mathrm{~Hz}, 1 \mathrm{H} ; A r), 8.48(\mathrm{~d}, \mathrm{~J}=3.6 \mathrm{~Hz}, 1 \mathrm{H} ; A r), 7.76(\mathrm{~m}, 1 \mathrm{H} ; A r)$, $7.76(\mathrm{~d}, J=7.6 \mathrm{~Hz}, 2 \mathrm{H} ; A r), 7.36(\mathrm{dd}, J=7.3 \mathrm{~Hz}, 7.3 \mathrm{~Hz}, 2 \mathrm{H} ; A r), 7.29-7.23(2 \mathrm{H} ; A r), 4.70-4.50(2 \mathrm{H}$; $\left.\mathrm{CH}_{2} \mathrm{CH}_{2} \mathrm{~F}\right), 2.98$ (brs, $\left.1 \mathrm{H} ; \mathrm{OH}\right), 2.82-2.73\left(2 \mathrm{H} ; \mathrm{CH}_{2} \mathrm{CH}_{2} \mathrm{~F}\right)$.

${ }^{13} \mathrm{C}$ NMR $\left(125 \mathrm{MHz}, \mathrm{CDCl}_{3}, \mathrm{rt}\right): \delta$ 148.1, 147.4, 145.5, 142.2, 134.1, 128.7, 127.6, 125.9, 123.3, 81.7 (d, J $=161.0 \mathrm{~Hz}), 76.0(\mathrm{~d}, J=6.0 \mathrm{~Hz}), 41.5(\mathrm{~d}, J=18.1 \mathrm{~Hz})$.

${ }^{19} \mathrm{~F} \mathrm{NMR}\left(376 \mathrm{MHz}, \mathrm{CDCl}_{3}\right.$, r.t.): $\delta-221.2\left(\mathrm{~m}, 1 \mathrm{~F} ; \mathrm{CH}_{2} \mathrm{~F}\right)$.

HRMS (ESI-TOF) calcd m/z for [ $\left.\mathrm{C}_{14} \mathrm{H}_{14} \mathrm{FNO}+\mathrm{Na}\right]^{+} 254.0952$ found 254.0954 . 
3-Fluoro-1-phenyl-1-(thiophen-2-yl)propan-1-ol (16I)<smiles>OC(CCF)(c1ccccc1)c1cccs1</smiles>

According to the general procedures (reaction time = 12 h), 2-(1-phenylvinyl)thiophene 15I (46.6 mg, 0.250 mmol), 3 (123 mg, $0.375 \mathrm{mmol}), 1$ (5.8 mg, $12.5 \mu \mathrm{mol})$, acetone $(4.5 \mathrm{~mL})$ and $\mathrm{H}_{2} \mathrm{O}(0.5 \mathrm{~mL})$ afforded $16 \mathrm{I}$ as a colorless oil $(30.7 \mathrm{mg}, 0.130 \mathrm{mmol}, 52 \%)$. Eluent: Hexane/ethyl acetate $=10: 1$.

${ }^{1} \mathrm{H}$ NMR $\left(400 \mathrm{MHz}, \mathrm{CDCl}_{3}\right.$, rt): $\delta$ 7.52-7.49 (2H; Ar), 7.38-7.24 (4H; Ar), 6.97-6.92 (2H; Ar), 4.69-4.48 $\left(2 \mathrm{H} ; \mathrm{CH}_{2} \mathrm{CH}_{2} \mathrm{~F}\right), 2.91$ (d, J = Hz, 1H; OH), 2.86-2.69 (2H; $\left.\mathrm{CH}_{2} \mathrm{CH}_{2} \mathrm{~F}\right)$.

${ }^{13} \mathrm{C} \mathrm{NMR}\left(125 \mathrm{MHz}, \mathrm{CDCl}_{3}, \mathrm{rt}\right): \delta 152.2,145.1,128.4,127.6,126.8,125.6,125.3,124.1,81.9$ (d, J = 160.5 $\mathrm{Hz}), 76.5(\mathrm{~d}, J=5.8 \mathrm{~Hz}), 43.4(\mathrm{~d}, J=18.0 \mathrm{~Hz})$.

${ }^{19} \mathrm{~F}$ NMR $\left(376 \mathrm{MHz}, \mathrm{CDCl}_{3}\right.$, r.t.): $\delta-221.2\left(\mathrm{~m}, 1 \mathrm{~F} ; \mathrm{CH}_{2} F\right)$.

HRMS (ESI-TOF) calcd $\mathrm{m} / \mathrm{z}$ for $\left[\mathrm{C}_{13} \mathrm{H}_{13} \mathrm{FOS}+\mathrm{Na}\right]^{+} 259.0563$ found 259.0561.

3-Fluoro-2-methyl-1,1-diphenylpropan-1-ol (16m)<smiles>CC(CF)C(O)(c1ccccc1)c1ccccc1</smiles>

According to the general procedures (reaction time $=24 \mathrm{~h}$ ), prop-1-ene-1,1-diyldibenzene $15 \mathrm{~m}$ (48.6 $\mathrm{mg}$, $0.250 \mathrm{mmol}$ ), 3 (123 mg, $0.375 \mathrm{mmol}), 1$ (9.3 mg, $20.1 \mu \mathrm{mol})$, acetone $(4.5 \mathrm{~mL})$ and $\mathrm{H}_{2} \mathrm{O}(0.5 \mathrm{~mL})$ afforded $16 \mathrm{~m}$ as a colorless oil (32.8 mg, $0.134 \mathrm{mmol}, 54 \%)$. Eluent: Hexane/ethyl acetate $=10: 1$.

${ }^{1} \mathrm{H}$ NMR $\left(400 \mathrm{MHz}, \mathrm{CDCl}_{3}\right.$, rt): $\delta 7.49$ (d, J = 8.3 Hz, 4H; $\left.A r\right), 7.31$ (dd, J=8.3 Hz, 8.3 Hz, 4H; Ar), 7.19 (t, $J=7.3 \mathrm{~Hz}, 2 \mathrm{H} ; \mathrm{Ar}), 4.56-4.37\left(2 \mathrm{H} ; \mathrm{CHCH}_{2} \mathrm{~F}\right), 3.01\left(\mathrm{~m}, 1 \mathrm{H} ; \mathrm{CHCH}_{2} \mathrm{~F}\right) 2.58(\mathrm{~d}, J=7.9 \mathrm{~Hz}, 1 \mathrm{H} ; \mathrm{OH}), 1.14$ $(\mathrm{d}, \mathrm{J}=6.9 \mathrm{~Hz}, 3 \mathrm{H} ; M e)$.

${ }^{13} \mathrm{C}$ NMR $\left(125 \mathrm{MHz}, \mathrm{CDCl}_{3}, \mathrm{rt}\right): \delta 146.1,145.8,128.5,128.4,126.91,126.86,125.6,125.4,87.3$ (d, $\mathrm{J}=$ 163.3 Hz), 79.9 (d, $J=5.3 \mathrm{~Hz}), 41.4(\mathrm{~d}, J=16.8 \mathrm{~Hz}), 12.0(\mathrm{~d}, J=3.6 \mathrm{~Hz})$.

${ }^{19} \mathrm{~F}$ NMR (376 MHz, $\mathrm{CDCl}_{3}$, r.t.): $\delta-229.5$ (m, $\left.1 \mathrm{~F} ; \mathrm{CH}_{2} \mathrm{~F}\right)$.

HRMS (ESI-TOF) calcd $\mathrm{m} / \mathrm{z}$ for $\left[\mathrm{C}_{16} \mathrm{H}_{17} \mathrm{FO}+\mathrm{Na}\right]^{+} 267.1156$ found 267.1151 . 
1-Fluoro-5-methyl-3,5-diphenylhexan-3-ol (18a)

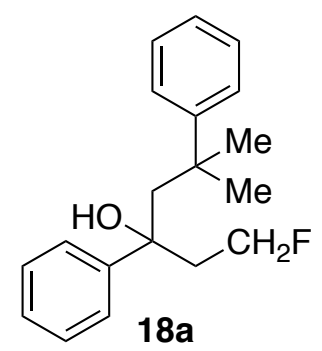

According to the general procedures (reaction time $=18 \mathrm{~h}$ ), 2,4-diphenyl-4-methyl-1-pentene $17 \mathrm{a}(57.6$ $\mathrm{mg}, 0.250 \mathrm{mmol}), 3$ (123 mg, $0.376 \mathrm{mmol}), 1(5.8 \mathrm{mg}, 12.5 \mu \mathrm{mol})$, acetone $(4.5 \mathrm{~mL})$ and $\mathrm{H}_{2} \mathrm{O}(0.5 \mathrm{~mL})$ afforded 18a as a colorless oil (42.3 mg, $0.148 \mathrm{mmol}, 61 \%)$. Eluent: Hexane/ethyl acetate = 10:1.

${ }^{1} \mathrm{H}$ NMR (400 MHz, $\mathrm{CDCl}_{3}$, rt): $\delta 7.31-7.19(10 \mathrm{H} ; A r), 4.40-4.04\left(2 \mathrm{H} ; \mathrm{CH}_{2} \mathrm{CH}_{2} \mathrm{~F}\right), 2.79(\mathrm{~d}, \mathrm{~J}=14.8 \mathrm{~Hz}, 1 \mathrm{H}$; $\mathrm{CHHCMe}_{2} \mathrm{Ph}$ ), 2.33 (d, J=14.8 Hz, 1H; CHHCMe $\left.2 \mathrm{Ph}\right), 2.22-1.93\left(2 \mathrm{H} ; \mathrm{CH}_{2} \mathrm{CH}_{2} \mathrm{~F}\right), 1.75(\mathrm{~d}, J=3.7 \mathrm{~Hz}, 1 \mathrm{H}$; $\mathrm{OH}), 1.25$ (s, 3H, $\left.\mathrm{CH}_{2} \mathrm{CMeMePh}\right), 1.04$ (s, 3H, $\mathrm{CH}_{2} \mathrm{CMeMePh}$ ).

${ }^{13} \mathrm{C}$ NMR $\left(125 \mathrm{MHz}, \mathrm{CDCl}_{3}, \mathrm{rt}\right): \delta 148.7,146.0,128.7,128.1,126.6,126.3,126.1,125.4,81.4$ (d, J = 159.8 $\mathrm{Hz}), 76.8(\mathrm{~d}, J=6.5 \mathrm{~Hz}), 55.9,45.3(\mathrm{~d}, J=17.8 \mathrm{~Hz}), 37.9,33.3,28.4$.

${ }^{19} \mathrm{~F}$ NMR (376 $\mathrm{MHz}, \mathrm{CDCl}_{3}$, r.t.): $\delta-221.3\left(\mathrm{~m}, 1 \mathrm{~F} ; \mathrm{CH}_{2} \mathrm{~F}\right)$.

HRMS (ESI-TOF) calcd m/z for [ $\left.\mathrm{C}_{19} \mathrm{H}_{23} \mathrm{FO}+\mathrm{Na}\right]^{+} 309.1625$ found 309.1626.

4-Fluoro-2-phenylbutan-2-ol (18b)<smiles>CC(O)(CCF)c1ccccc1</smiles>

According to the general procedures (reaction time $=18 \mathrm{~h}$ ), $\alpha$-methylstyrene $17 \mathrm{~b}(29.5 \mathrm{mg}, 0.250 \mathrm{mmol})$, 3 (123 mg, $0.375 \mathrm{mmol}), 1(5.8 \mathrm{mg}, 12.5 \mu \mathrm{mol})$, acetone $(4.5 \mathrm{~mL})$ and $\mathrm{H}_{2} \mathrm{O}(0.5 \mathrm{~mL})$ afforded $18 \mathrm{~b}$ as a colorless oil (23.0 mg, $0.137 \mathrm{mmol}, 55 \%)$. Eluent: Hexane/ethyl acetate $=4: 1$.

${ }^{1} \mathrm{H}$ NMR (400 MHz, $\left.\mathrm{CDCl}_{3}, \mathrm{rt}\right): \delta 7.46-7.25(5 \mathrm{H} ; \mathrm{Ar}), 4.61-4.39\left(2 \mathrm{H} ; \mathrm{CH}_{2} \mathrm{CH}_{2} \mathrm{~F}\right), 2.36-2.13\left(3 \mathrm{H} ; \mathrm{CH}_{2} \mathrm{CH}_{2} \mathrm{~F}\right.$, $\mathrm{OH}), 1.62(\mathrm{~s}, 3 \mathrm{H}, \mathrm{Me})$.

${ }^{13} \mathrm{C}$ NMR $\left(125 \mathrm{MHz}, \mathrm{CDCl}_{3}, \mathrm{rt}\right): \delta 147.2,128.5,127.0,124.7,82.0(\mathrm{~d}, J=160.5 \mathrm{~Hz}), 74.1(\mathrm{~d}, J=4.2 \mathrm{~Hz})$, $43.7(\mathrm{~d}, \mathrm{~J}=17.7 \mathrm{~Hz}), 30.7$.

${ }^{19} \mathrm{~F}$ NMR (376 MHz, $\mathrm{CDCl}_{3}$, r.t.): $\delta-220.5$ (m, $1 \mathrm{~F} ; \mathrm{CH}_{2} F$ ).

HRMS (ESI-TOF) calcd $\mathrm{m} / \mathrm{z}$ for $\left[\mathrm{C}_{10} \mathrm{H}_{13} \mathrm{FO}+\mathrm{Na}\right]^{+} 191.0843$ found 191.0842. 
1-Fluoro-4-methyl-3-phenylpentan-3-ol (18c)<smiles>CC(C)C(O)(CCF)c1ccccc1</smiles>

According to the general procedures (reaction time $=18 \mathrm{~h}$ ), (3-methylbut-1-en-2-yl)benzene 17c (46.6 mg, $0.250 \mathrm{mmol}$ ), 3 (123 mg, $0.377 \mathrm{mmol}), 1(5.8 \mathrm{mg}, 12.5 \mu \mathrm{mol})$, acetone $(4.5 \mathrm{~mL})$ and $\mathrm{H}_{2} \mathrm{O}(0.5 \mathrm{~mL})$ afforded $18 \mathrm{c}$ as a colorless oil $(27.9 \mathrm{mg}, 0.142 \mathrm{mmol}, 57 \%)$. Eluent: Hexane/ethyl acetate $=10: 1$.

${ }^{1} \mathrm{H}$ NMR $\left(400 \mathrm{MHz}, \mathrm{CDCl}_{3}, \mathrm{rt}\right): \delta$ 7.38-7.32 $(4 \mathrm{H} ; \mathrm{Ar}), 7.24(\mathrm{~m}, 1 \mathrm{H} ; \mathrm{Ar}), 4.46-4.31\left(2 \mathrm{H} ; \mathrm{CH}_{2} \mathrm{CH}_{2} \mathrm{~F}\right), 2.45(\mathrm{~m}$, $1 \mathrm{H} ; \mathrm{CHMe}_{2}$ ), 2.26-2.00 (3H; $\left.\mathrm{CH}_{2} \mathrm{CH}_{2} \mathrm{~F}, \mathrm{OH}\right), 1.01$ (d, $\left.J=6.8 \mathrm{~Hz}, 3 \mathrm{H}, \mathrm{CHMeMe}\right), 0.71$ (d, $J=6.8 \mathrm{~Hz}, 3 \mathrm{H}$, CHMeMe).

${ }^{13} \mathrm{C}$ NMR $\left(125 \mathrm{MHz}, \mathrm{CDCl}_{3}, \mathrm{rt}\right): \delta 144.6,128.2,126.7,125.7,82.6(\mathrm{~d}, J=159.5 \mathrm{~Hz}), 78.4(\mathrm{~d}, J=5.0 \mathrm{~Hz})$, $39.7(\mathrm{~d}, J=17.6 \mathrm{~Hz}), 38.6,17.3,16.7$.

${ }^{19} \mathrm{~F}$ NMR (376 MHz, $\mathrm{CDCl}_{3}$, r.t.): $\delta-220.6\left(\mathrm{~m}, 1 \mathrm{~F} ; \mathrm{CH}_{2} \mathrm{~F}\right)$.

HRMS (ESI-TOF) calcd $\mathrm{m} / \mathrm{z}$ for $\left[\mathrm{C}_{12} \mathrm{H}_{17} \mathrm{FO}+\mathrm{Na}\right]^{+} 219.1156$ found 219.1157.

1-Cyclohexyl-3-fluoro-1-phenylpropan-1-ol (18d)<smiles>OC(CCF)(c1ccccc1)C1CCCCC1</smiles>

According to the general procedures (reaction time $=18 \mathrm{~h}$ ), (1-cyclohexylvinyl)benzene $17 \mathrm{~d}(46.6 \mathrm{mg}$, $0.250 \mathrm{mmol}$ ), 3 (123 mg, $0.377 \mathrm{mmol}), 1(5.8 \mathrm{mg}, 12.5 \mu \mathrm{mol})$, acetone $(4.5 \mathrm{~mL})$ and $\mathrm{H}_{2} \mathrm{O}(0.5 \mathrm{~mL})$ afforded $18 \mathrm{~d}$ as a colorless oil $(38.9 \mathrm{mg}, 0.165 \mathrm{mmol}, 66 \%)$. Eluent: Hexane/ethyl acetate $=10: 1$.

${ }^{1} \mathrm{H}$ NMR (400 MHz, $\left.\mathrm{CDCl}_{3}, \mathrm{rt}\right): \delta 7.36-7.31(4 \mathrm{H} ; \mathrm{Ar}), 7.24(\mathrm{~m}, 1 \mathrm{H} ; \mathrm{Ar}), 4.49-4.29\left(2 \mathrm{H} ; \mathrm{CH}_{2} \mathrm{CH}_{2} \mathrm{~F}\right), 2.51-$ $2.12\left(3 \mathrm{H} ; \mathrm{CH}_{2} \mathrm{CH}_{2} \mathrm{~F}, \mathrm{OH}\right), 1.97-1.60(5 \mathrm{H}$, cyclohexyl's $H)$, 1.37-0.92 (6H, cyclohexyl's $\left.H\right)$.

${ }^{13} \mathrm{C}$ NMR $\left(125 \mathrm{MHz}, \mathrm{CDCl}_{3}, \mathrm{rt}\right): \delta 144.6,128.1,126.6,125.8,82.6$ (d, J = 159.2 Hz), 78.4 (d, J = 5.0 Hz), 48.9, 39.3 (d, $J=18.1 \mathrm{~Hz}), 27.1,26.71,26.69(2 \mathrm{C}), 26.5$.

${ }^{19} \mathrm{~F}$ NMR (376 MHz, $\mathrm{CDCl}_{3}$, r.t.): $\delta-220.6\left(\mathrm{~m}, 1 \mathrm{~F} ; \mathrm{CH}_{2} \mathrm{~F}\right)$.

HRMS (ESI-TOF) calcd m/z for [ $\left.\mathrm{C}_{15} \mathrm{H}_{21} \mathrm{FO}+\mathrm{Na}\right]^{+} 259.1469$ found 259.1466.

1-(2-Fluoroethyl)-2,3-dihydro-1H-inden-1-ol (20a)

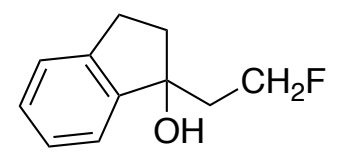

20a

According to the general procedures (reaction time $=18 \mathrm{~h}), 1$-methylene-2,3-dihydro- $1 \mathrm{H}$-indene $19 \mathrm{a}(32.5$ 
$\mathrm{mg}, 0.250 \mathrm{mmol}), 3(123 \mathrm{mg}, 0.374 \mathrm{mmol}), 1(5.8 \mathrm{mg}, 12.5 \mu \mathrm{mol})$, acetone $(4.5 \mathrm{~mL})$ and $\mathrm{H}_{2} \mathrm{O}(0.5 \mathrm{~mL})$ afforded 20a as a colorless oil $(27.1 \mathrm{mg}, 0.150 \mathrm{mmol}, 60 \%)$. Eluent: Hexane/ethyl acetate $=4: 1$.

${ }^{1} \mathrm{H}$ NMR $\left(400 \mathrm{MHz}, \mathrm{CDCl}_{3}, \mathrm{rt}\right): \delta 7.34(\mathrm{~m}, 1 \mathrm{H} ; \mathrm{Ar}), 7.29-7.23(3 \mathrm{H} ; \mathrm{Ar}), 4.84-4.56\left(2 \mathrm{H} ; \mathrm{CH}_{2} \mathrm{CH}_{2} \mathrm{~F}\right), 3.07-$ $2.81\left(2 \mathrm{H} ; \mathrm{ArCH}_{2} \mathrm{CH}_{2}\right), 2.42-2.11\left(4 \mathrm{H} ; \mathrm{ArCH}_{2} \mathrm{CH}_{2}, \mathrm{CH}_{2} \mathrm{CH}_{2} \mathrm{~F}\right), 1.99$ (d, $\left.J=2.5 \mathrm{~Hz}, 1 \mathrm{H} ; \mathrm{OH}\right)$.

${ }^{13} \mathrm{C}$ NMR $\left(125 \mathrm{MHz}, \mathrm{CDCl}_{3}, \mathrm{rt}\right): \delta 146.9,143.0,128.6,127.0,125.2,122.8,82.4(\mathrm{~d}, J=4.2 \mathrm{~Hz}), 81.7$ (d, $J$ $=161.8 \mathrm{~Hz}), 40.6,40.0$ (d, $J=18.4 \mathrm{~Hz}), 29.5$.

${ }^{19} \mathrm{~F}$ NMR (376 MHz, $\mathrm{CDCl}_{3}$, r.t.): $\delta-219.6\left(\mathrm{~m}, 1 \mathrm{~F} ; \mathrm{CH}_{2} \mathrm{~F}\right)$.

HRMS (ESI-TOF) calcd $\mathrm{m} / \mathrm{z}$ for $\left[\mathrm{C}_{11} \mathrm{H}_{13} \mathrm{FO}+\mathrm{Na}\right]^{+} 203.0843$ found 203.0840.

1-(2-Fluoroethyl)-1,2,3,4-tetrahydronaphthalen-1-ol (20b)

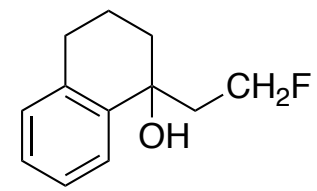

20b

According to the general procedures (reaction time $=18 \mathrm{~h}$ ), 1-methylene-1,2,3,4-tetrahydronaphthalene $19 \mathrm{~b}$ (36.1 mg, $0.250 \mathrm{mmol}), 3$ (123 mg, $0.376 \mathrm{mmol}), 1$ (5.8 mg, $12.5 \mu \mathrm{mol})$, acetone $(4.5 \mathrm{~mL})$ and $\mathrm{H}_{2} \mathrm{O}$ $(0.5 \mathrm{~mL})$ afforded $20 \mathrm{~b}$ as a colorless oil $(35.1 \mathrm{mg}, 0.181 \mathrm{mmol}, 72 \%)$. Eluent: Hexane/ethyl acetate $=4: 1$. ${ }^{1} \mathrm{H}$ NMR $\left(400 \mathrm{MHz}, \mathrm{CDCl}_{3}, \mathrm{rt}\right): \delta 7.53(\mathrm{~m}, 1 \mathrm{H} ; A r), 7.24-7.17(2 \mathrm{H} ; A r), 7.09(\mathrm{~d}, \mathrm{~J}=7.1 \mathrm{~Hz}, 1 \mathrm{H} ; A r), 4.80-$ $4.52\left(2 \mathrm{H} ; \mathrm{CH}_{2} \mathrm{CH} \mathrm{H}_{2} \mathrm{~F}\right), 2.88-2.74\left(2 \mathrm{H} ; \mathrm{ArCH}_{2} \mathrm{CH}_{2}\right), 2.37-1.84\left(7 \mathrm{H} ; \mathrm{ArCH}_{2} \mathrm{CH}_{2}, \mathrm{CH}_{2} \mathrm{C}, \mathrm{CH}_{2} \mathrm{CH}_{2} \mathrm{~F}, \mathrm{OH}\right)$.

${ }^{13} \mathrm{C} \mathrm{NMR}\left(125 \mathrm{MHz}, \mathrm{CDCl}_{3}, \mathrm{rt}\right): \delta 141.9,136.7,129.2,127.5,126.5,126.2,81.4$ (d, $\left.J=161.7 \mathrm{~Hz}\right), 71.7$ (d, $J=4.4 \mathrm{~Hz}$ ), $42.2(\mathrm{~d}, J=18.2 \mathrm{~Hz}), 36.7,29.7,19.9$.

${ }^{19} \mathrm{~F}$ NMR (376 MHz, $\mathrm{CDCl}_{3}$, r.t.): $\delta-219.0\left(\mathrm{~m}, 1 \mathrm{~F} ; \mathrm{CH}_{2} \mathrm{~F}\right)$.

HRMS (ESI-TOF) calcd m/z for [ $\left.\mathrm{C}_{12} \mathrm{H}_{15} \mathrm{FO}+\mathrm{Na}\right]^{+} 217.0999$ found 217.0999 .

4-(2-Fluoroethyl)thiochroman-4-ol (20c)<smiles>OC1(CCF)CCSc2ccccc21</smiles>

20c

According to the general procedures (reaction time $=18 \mathrm{~h}$ ), 4-methylenethiochromane $19 \mathrm{c}(40.6 \mathrm{mg}, 0.250$ mmol), 3 (123 mg, $0.377 \mathrm{mmol}), 1$ (5.8 mg, $12.5 \mu \mathrm{mol}$ ), acetone (4.5 mL) and $\mathrm{H}_{2} \mathrm{O}(0.5 \mathrm{~mL})$ afforded 20c as a colorless oil $(40.5 \mathrm{mg}, 0.191 \mathrm{mmol}, 76 \%)$. Eluent: Hexane/ethyl acetate $=4: 1$.

${ }^{1} \mathrm{H}$ NMR $\left(400 \mathrm{MHz}, \mathrm{CDCl}_{3}, \mathrm{rt}\right): \delta 7.69(\mathrm{~m}, 1 \mathrm{H} ; \mathrm{Ar}), 7.15-7.08(3 \mathrm{H} ; \mathrm{Ar}), 4.81-4.50\left(2 \mathrm{H} ; \mathrm{CH}_{2} \mathrm{CH}_{2} \mathrm{~F}\right), 3.16-$ $3.03\left(2 \mathrm{H} ; \mathrm{SCH}_{2} \mathrm{CH}_{2}\right), 2.43-2.10\left(4 \mathrm{H} ; \mathrm{SCH}_{2} \mathrm{CH}_{2}, \mathrm{CH}_{2} \mathrm{CH}_{2} \mathrm{~F}\right), 2.14$ (brs, $1 \mathrm{H} ; \mathrm{OH}$ ).

${ }^{13} \mathrm{C}$ NMR $\left(125 \mathrm{MHz}, \mathrm{CDCl}_{3}, \mathrm{rt}\right): \delta 139.2,132.7,127.9,126.6,125.9,124.5,81.1$ (d, J=161.6 Hz), 71.0 (d, $J=3.3 \mathrm{~Hz}$ ), 39.9 (d, $J=18.2 \mathrm{~Hz}), 35.5,23.3$.

${ }^{19} \mathrm{~F}$ NMR (376 $\mathrm{MHz}, \mathrm{CDCl}_{3}$, r.t.): $\delta-218.9\left(\mathrm{~m}, 1 \mathrm{~F} ; \mathrm{CH}_{2} \mathrm{~F}\right)$.

HRMS (ESI-TOF) calcd $\mathrm{m} / \mathrm{z}$ for $\left[\mathrm{C}_{11} \mathrm{H}_{12} \mathrm{FOS}+\mathrm{Na}\right]^{+} 235.0563$ found 235.0560 . 
(8R,9S,13S,14S)-3-(3-Fluoro-1-hydroxypropyl)-13-methyl-6,7,8,9,11,12,13,14,15,16-decahydro-17Hcyclopenta[a]phenanthren-17-one (22)

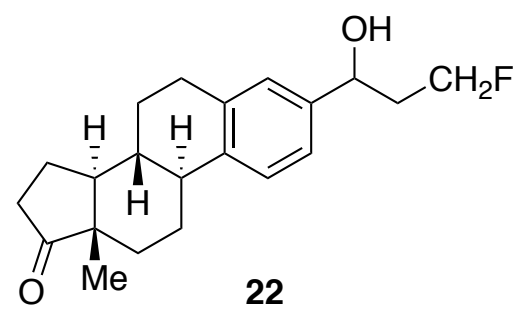

According to the general procedures (reaction time $=12 \mathrm{~h}),(8 R, 9 S, 13 S, 14 S)$-13-methyl-3-vinyl6,7,8,9,11,12,13,14,15,16-decahydro-17H-cyclopenta[a]phenanthren-17-one 21 (70.1 mg, $0.250 \mathrm{mmol}$ ), 3 (123 mg, $0.376 \mathrm{mmol}), 1(5.8 \mathrm{mg}, 12.5 \mu \mathrm{mol})$, acetone $(4.5 \mathrm{~mL})$ and $\mathrm{H}_{2} \mathrm{O}(0.5 \mathrm{~mL})$ afforded 22 as a colorless oil (24.7 mg, $0.0747 \mathrm{mmol}, 30 \%)$. Eluent: Hexane/ethyl acetate $=1: 1$.

${ }^{1} \mathrm{H}$ NMR (400 MHz, CDCl 3 , rt): $\delta 7.30$ (d, J = Hz, 1H; $\left.A r\right), 7.15$ (d, J = Hz, 1H; $\left.A r\right), 7.12(\mathrm{~s}, 1 \mathrm{H} ; A r), 4.87$ (m, $\left.1 \mathrm{H} ; \mathrm{CH}_{2} \mathrm{CHOH}\right), 4.78-4.44\left(2 \mathrm{H} ; \mathrm{CH}_{2} \mathrm{~F}\right), 2.95-2.91$ (2H; steroid's $\left.H\right), 2.54-2.42(2 \mathrm{H}$; steroid's H), 2.31 (m, 1H; steroid's H), 2.23-1.95 (7H; $\mathrm{CH}_{2} \mathrm{CHOH}, \mathrm{OH}$, steroid's H), 1.69-1.40 (6H; steroid's H), 0.91 (s, 3H; Me).

${ }^{13} \mathrm{C}$ NMR $\left(125 \mathrm{MHz}_{2} \mathrm{CDCl}_{3}, \mathrm{rt}\right): \delta 221.1,141.6,139.5,136.9,126.5$ (apparent d), 125.8 (apparent d), 123.3, $81.6(\mathrm{~d}, J=162.1 \mathrm{~Hz}), 70.7,50.6,48.1,44.5,39.6(\mathrm{~d}, J=19.6 \mathrm{~Hz}$ ), 38.3, 36.0, 31.7, 29.6 (apparent d), 26.6, 25.8, 21.7, 13.9 .

${ }^{19} \mathrm{~F}$ NMR (376 MHz, $\mathrm{CDCl}_{3}$, r.t.): $\delta-222.3\left(\mathrm{~m}, 1 \mathrm{~F} ; \mathrm{CH}_{2} \mathrm{~F}\right)$.

HRMS (ESI-TOF) calcd $\mathrm{m} / \mathrm{z}$ for $\left[\mathrm{C}_{21} \mathrm{H}_{27} \mathrm{FO}_{2}+\mathrm{Na}\right]^{+} 353.1887$ found 353.1886 .

4-(2-Fluoroethyl)-2-phenylchroman-4-ol (24)<smiles>CCC1(O)CC(c2ccccc2)Oc2ccccc21</smiles>

According to the general procedures (reaction time $=24 \mathrm{~h}$ ), 4-methylene-2-phenylchromane 23 (55.6 mg, $0.250 \mathrm{mmol}$ ), 3 (123 mg, $0.376 \mathrm{mmol}), 1(5.8 \mathrm{mg}, 12.5 \mu \mathrm{mol})$, acetone $(4.5 \mathrm{~mL})$ and $\mathrm{H}_{2} \mathrm{O}(0.5 \mathrm{~mL})$ afforded 24 as a colorless oil (50.5 mg, $0.185 \mathrm{mmol}, 74 \%)$. Eluent: Hexane/ethyl acetate $=4: 1$.

${ }^{1} \mathrm{H}$ NMR $\left(400 \mathrm{MHz}, \mathrm{CDCl}_{3}, \mathrm{rt}\right): \delta 7.50-7.33(6 \mathrm{H} ; A r), 7.27(\mathrm{~m}, 1 \mathrm{H} ; A r), 7.03-6.99(2 \mathrm{H} ; A r), 5.23$ (dd, J=11.9 $\left.\mathrm{Hz}, 1.7 \mathrm{~Hz}, 1 \mathrm{H} ; \mathrm{OCHCH}_{2}\right), 4.77-4.51\left(2 \mathrm{H} ; \mathrm{CH}_{2} \mathrm{CH}_{2} \mathrm{~F}\right), 2.63\left(\mathrm{~m}, 1 \mathrm{H} ; \mathrm{CHHCH}_{2} \mathrm{~F}\right), 2.32-2.14\left(4 \mathrm{H} ; \mathrm{CH} \mathrm{HCH}_{2} \mathrm{~F}\right.$, $\mathrm{OH}, \mathrm{OCHCH} \mathrm{H}_{2}$.

${ }^{13} \mathrm{C}$ NMR $\left(125 \mathrm{MHz}, \mathrm{CDCl}_{3}, \mathrm{rt}\right): \delta 155.0,140.9,130.0,128.7,128.2,126.34,126.25,126.0,121.4,118.2$, 81.1 (d, $J=163.0 \mathrm{~Hz}), 74.4,68.3(\mathrm{~d}, J=3.6 \mathrm{~Hz}), 43.2$ (d, $J=0.5 \mathrm{~Hz}), 41.4$ (d, $J=18.7 \mathrm{~Hz})$.

${ }^{19} \mathrm{~F}$ NMR (376 MHz, $\mathrm{CDCl}_{3}$, r.t.): $\delta-218.5$ (m, $\left.1 \mathrm{~F} ; \mathrm{CH}_{2} \mathrm{~F}\right)$.

HRMS (ESI-TOF) calcd $\mathrm{m} / \mathrm{z}$ for $\left[\mathrm{C}_{17} \mathrm{H}_{17} \mathrm{FO}_{2}+\mathrm{Na}\right]^{+} 295.1105$ found 295.1105. 
4-\{3-Fluoro-1-hydroxy-1-(3,5,5,8,8-pentamethyl-5,6,7,8-tetrahydronaphthalen-2-yl)propyl\}benzoic acid (26)<smiles>CCC(O)(c1ccc(C(=O)O)cc1)c1cc2c(cc1[N+](=O)[O-])C(C)(C)CCC2(C)C</smiles>

According to the general procedures (reaction time $=24 \mathrm{~h})$, bexarotene $25(87.1 \mathrm{mg}, 0.250 \mathrm{mmol}), \mathbf{3}(123$ $\mathrm{mg}, 0.376 \mathrm{mmol}), 1(5.8 \mathrm{mg}, 12.5 \mu \mathrm{mol})$, acetone $(4.5 \mathrm{~mL})$ and $\mathrm{H}_{2} \mathrm{O}(0.5 \mathrm{~mL})$ afforded 26 as a white solid (45.2 $\mathrm{mg}, 0.113 \mathrm{mmol}, 45 \%$ ) after purification by flash column chromatography on silica-gel (hexane/ethyl acetate $=1: 1$ ) recycling preparative HPLC and reprecipitation with $\mathrm{CH}_{2} \mathrm{Cl}_{2}$ and hexane.

${ }^{1} \mathrm{H}$ NMR (400 MHz, CDCl 3 , rt): $\delta 11.65$ (brs, $1 \mathrm{H}, \mathrm{COOH}$ ), 8.04 (d, J = 8.4 Hz, 2H; Ar), 7.51 (s, $\left.1 \mathrm{H} ; A r\right), 7.42$ $(\mathrm{d}, J=8.4 \mathrm{~Hz}, 2 \mathrm{H} ; A r), 6.99(\mathrm{~s}, 1 \mathrm{H} ; \mathrm{Ar}), 4.69-4.37\left(2 \mathrm{H} ; \mathrm{CH}_{2} \mathrm{CH}_{2} \mathrm{~F}\right), 2.86-2.62\left(2 \mathrm{H} ; \mathrm{CH}_{2} \mathrm{CH}_{2} \mathrm{~F}\right), 2.49$ (brs, $1 \mathrm{H} ; \mathrm{OH}), 1.92(\mathrm{~s}, 3 \mathrm{H} ; \mathrm{ArMe}), 1.72-1.68(4 \mathrm{H}$; methylene' $\mathrm{H}), 1.33(6 \mathrm{H} ; \mathrm{Me}), 1.26(\mathrm{~s}, 3 \mathrm{H} ; \mathrm{Me}), 1.25(\mathrm{~s}, 3 \mathrm{H}$; $\mathrm{Me})$.

${ }^{13} \mathrm{C}$ NMR $\left(125 \mathrm{MHz}, \mathrm{CDCl}_{3}, \mathrm{rt}\right): \delta 172.1,152.1,144.7,141.9,139.8,133.9,131.1,130.2,127.9,126.2$, 123.9, $81.8(\mathrm{~d}, J=160.5 \mathrm{~Hz}), 78.0(\mathrm{~d}, J=7.1 \mathrm{~Hz}), 42.7(\mathrm{~d}, J=18.8 \mathrm{~Hz}), 35.3,35.2,34.2,34.0,32.2,32.1$, $31.9,31.7,21.1$.

${ }^{19} \mathrm{~F}$ NMR $\left(376 \mathrm{MHz}, \mathrm{CDCl}_{3}\right.$, r.t. $): \delta-221.2\left(\mathrm{~m}, 1 \mathrm{~F} ; \mathrm{CH}_{2} F\right)$.

HRMS (ESI-TOF) calcd $\mathrm{m} / \mathrm{z}$ for $\left[\mathrm{C}_{25} \mathrm{H}_{31} \mathrm{FO}_{3}+\mathrm{Na}\right]^{+} 421.2149$ found 421.2147 .

Melting point: $186^{\circ} \mathrm{C}$.

\section{Procedures for Other Monofluoromethylation and Characterization}

3-Fluoro-1-methoxy-1,1-diphenylpropane (27a)<smiles>[14CH3]C=C(c1ccccc1)c1ccccc1</smiles>

$15 a$<smiles></smiles>

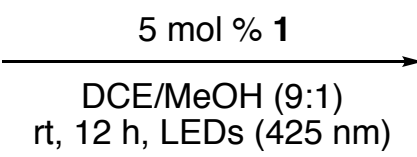

$\mathrm{rt}, 12 \mathrm{~h}$, LEDs (425 nm)<smiles>CCC(OC)(c1ccccc1)c1ccccc1</smiles>

$27 a$

A $20 \mathrm{~mL}$ Schlenk tube was charged with 1,1-diphenylethylene $15 \mathrm{a}(45.1 \mathrm{mg}, 0.250 \mathrm{mmol}), 3$ (123 mg, $0.376 \mathrm{mmol}), 1(5.8 \mathrm{mg}, 12.5 \mu \mathrm{mol}), 1,2$-dichloroethane $(4.5 \mathrm{~mL})$ and $\mathrm{MeOH}(0.5 \mathrm{~mL})$. The mixture was degassed by three freeze-pump-thaw cycles. The Schlenk tube was placed at 2-3 cm away from blue LED lamps $(\lambda=425 \mathrm{~nm})$ in a water bath. The mixture was stirred for $12 \mathrm{~h}$ at room temperature under visible light irradiation. After the reaction, the reaction mixture was concentrated under reduced pressure. The desired product 27 a was obtained as a colorless oil $(46.1 \mathrm{mg}, 0.189 \mathrm{mmol}, 75 \%)$ after purification by flash column chromatography on silica-gel (hexane/ethyl acetate $=20: 1$ ) and recycling preparative HPLC. ${ }^{1} \mathrm{H}$ NMR $\left(400 \mathrm{MHz}, \mathrm{CDCl}_{3}, \mathrm{rt}\right): \delta 7.35-7.21(10 \mathrm{H} ; \mathrm{Ar}), 4.36\left(\mathrm{dt}, \mathrm{J}=47.0 \mathrm{~Hz}, 7.6 \mathrm{~Hz}, 2 \mathrm{H} ; \mathrm{CH}_{2} \mathrm{CH}_{2} \mathrm{~F}\right), 3.11$ (s, 3H; OMe), 2.82 (dt, J=14.9 Hz, 7.6 Hz, 2H; $\mathrm{CH}_{2} \mathrm{CH}_{2} \mathrm{~F}$ ). 
${ }^{13} \mathrm{C}$ NMR $\left(125 \mathrm{MHz}, \mathrm{CDCl}_{3}, \mathrm{rt}\right): \delta 144.7,128.3,127.2,126.7,81.13(\mathrm{~d}, J=160.8 \mathrm{~Hz}), 81.10(\mathrm{~d}, J=10.2$ $\mathrm{Hz}), 50.5,35.3(\mathrm{~d}, J=19.6 \mathrm{~Hz})$.

${ }^{19} \mathrm{~F}$ NMR (376 MHz, $\mathrm{CDCl}_{3}$, r.t.): $\delta-223.9\left(\mathrm{~m}, 1 \mathrm{~F} ; \mathrm{CH}_{2} \mathrm{~F}\right)$.

HRMS (ESI-TOF) calcd $\mathrm{m} / \mathrm{z}$ for $\left[\mathrm{C}_{16} \mathrm{H}_{17} \mathrm{FO}+\mathrm{Na}\right]^{+} 267.1156$ found 267.1153.

3-Fluoro-1,1-diphenylprop-1-ene (28a)

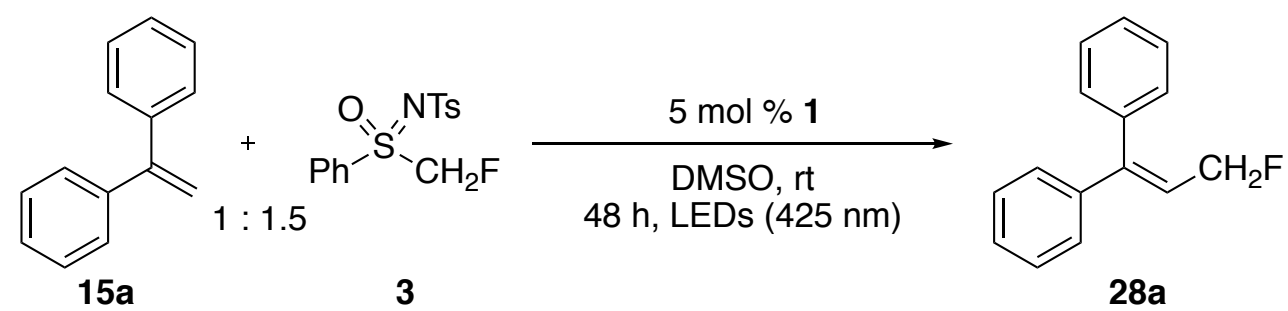

A $20 \mathrm{~mL}$ Schlenk tube was charged with 1,1-diphenylethylene 15a (45.1 mg, $0.250 \mathrm{mmol}), 3$ (123 mg, $0.375 \mathrm{mmol}), 1(5.8 \mathrm{mg}, 12.5 \mu \mathrm{mol})$, dimethyl sulfoxide $(5.0 \mathrm{~mL})$. The mixture was degassed by three freeze-pump-thaw cycles. The Schlenk tube was placed at $2-3 \mathrm{~cm}$ away from blue LED lamps $(\lambda=425$ $\mathrm{nm}$ ) in a water bath. The mixture was stirred for $48 \mathrm{~h}$ at room temperature under visible light irradiation. After the reaction, water was added and the mixture was extracted with diethyl ether. The organic layer was dried over $\mathrm{Na}_{2} \mathrm{SO}_{4}$, filtered and evaporated. The desired product 28a was obtained as a colorless oil $(27.8 \mathrm{mg}, 0.131 \mathrm{mmol}, 52 \%)$ after purification by flash column chromatography on silica-gel (pentane) and recycling preparative HPLC.

${ }^{1} \mathrm{H}$ NMR (400 MHz, $\mathrm{CDCl}_{3}$, rt): $\delta$ 7.41-7.36 (3H; $\left.A r\right), 7.31-7.27(5 \mathrm{H} ; A r), 7.19-7.16(2 \mathrm{H} ; A r), 6.30(\mathrm{dt}, J=$ $\left.10.6 \mathrm{~Hz}, 7.1 \mathrm{~Hz}, 1 \mathrm{H} ; \mathrm{C}=\mathrm{CHCH}_{2} \mathrm{~F}\right), 4.92\left(\mathrm{dd}, J=47.0 \mathrm{~Hz}, 7.1 \mathrm{~Hz}, 2 \mathrm{H} ; \mathrm{C}=\mathrm{CHCH}_{2} \mathrm{~F}\right.$ ).

${ }^{13} \mathrm{C} \mathrm{NMR}\left(125 \mathrm{MHz}, \mathrm{CDCl}_{3}, \mathrm{rt}\right): \delta 147.7$ (d, $\left.J=11.9 \mathrm{~Hz}\right), 141.4(\mathrm{~d}, J=2.8 \mathrm{~Hz}), 138.5(\mathrm{~d}, J=3.2 \mathrm{~Hz}), 129.9$ $(\mathrm{d}, J=2.2 \mathrm{~Hz}), 128.41,128.40,128.3,128.1,128.0(\mathrm{~d}, J=2.3 \mathrm{~Hz}), 122.7(\mathrm{~d}, J=18.0 \mathrm{~Hz}), 80.7(\mathrm{~d}, J=$ $156.9 \mathrm{~Hz})$.

${ }^{19} \mathrm{~F} \mathrm{NMR}\left(376 \mathrm{MHz}, \mathrm{CDCl}_{3}\right.$, r.t.): $\delta-207.2$ (dt, $\left.J=46.6 \mathrm{~Hz}, 10.7 \mathrm{~Hz}, 1 \mathrm{~F} ; \mathrm{CH}_{2} F\right)$.

HRMS (ESI-TOF) calcd $\mathrm{m} / \mathrm{z}$ for $\left[\mathrm{C}_{15} \mathrm{H}_{13} \mathrm{~F}+\mathrm{Na}\right]^{+} 235.0893$ found 235.0896 .

5-(2-Fluoroethyl)-5-phenyldihydrofuran-2(3H)-one (30)<smiles>C=C(CCC(=O)O)c1ccccc1</smiles>

A $20 \mathrm{~mL}$ Schlenk tube was charged with 4-phenylpent-4-enoic acid 29 (44.0 mg, $0.250 \mathrm{mmol}), 3$ (123 mg, $0.377 \mathrm{mmol}), 1(5.8 \mathrm{mg}, 12.5 \mu \mathrm{mol}), \mathrm{K}_{2} \mathrm{HPO}_{4}(87.1 \mathrm{mg}, 0.500 \mathrm{mmol})$, acetonitrile $(5.0 \mathrm{~mL})$. The mixture was degassed by three freeze-pump-thaw cycles. The Schlenk tube was placed at 2-3 cm away from blue LED lamps $(\lambda=425 \mathrm{~nm})$ in a water bath. The mixture was stirred for $24 \mathrm{~h}$ at room temperature under visible light irradiation. After the reaction, water was added and the mixture was extracted with $\mathrm{CH}_{2} \mathrm{Cl}_{2}$. The organic layer was dried over $\mathrm{Na}_{2} \mathrm{SO}_{4}$, filtered and evaporated. the reaction mixture was concentrated 
under reduced pressure. The desired product 30 was obtained as a colorless oil $(32.9 \mathrm{mg}, 0.158 \mathrm{mmol}$, $63 \%$ ) after purification by flash column chromatography on silica-gel (hexane/ethyl acetate $=4: 1$ ) and recycling preparative HPLC.

${ }^{1} \mathrm{H}$ NMR $\left(400 \mathrm{MHz}, \mathrm{CDCl}_{3}, \mathrm{rt}\right): \delta 7.42-7.31(5 \mathrm{H} ; \mathrm{Ar}), 4.64-4.21\left(2 \mathrm{H} ; \mathrm{CH}_{2} \mathrm{CH}_{2} \mathrm{~F}\right), 2.65-2.30\left(6 \mathrm{H} ; \mathrm{CH}_{2} \mathrm{CH}_{2} \mathrm{~F}\right.$, $\mathrm{CH}_{2} \mathrm{CH}_{2} \mathrm{CO}, \mathrm{CH}_{2} \mathrm{CH}_{2} \mathrm{CO}$ ).

${ }^{13} \mathrm{C}$ NMR $\left(125 \mathrm{MHz}, \mathrm{CDCl}_{3}, \mathrm{rt}\right): \delta 176.3,142.0,128.9,128.2,124.6,87.4(\mathrm{~d}, J=5.0 \mathrm{~Hz}), 80.0(\mathrm{~d}, J=164.2$ $\mathrm{Hz}), 42.3(\mathrm{~d}, J=19.4 \mathrm{~Hz}), 35.1$ (d, $J=0.4 \mathrm{~Hz}), 28.4$.

${ }^{19} \mathrm{~F}$ NMR (376 MHz, $\mathrm{CDCl}_{3}$, r.t.): $\delta-221.2\left(\mathrm{~m}, 1 \mathrm{~F} ; \mathrm{CH}_{2} \mathrm{~F}\right)$.

HRMS (ESI-TOF) calcd $\mathrm{m} / \mathrm{z}$ for $\left[\mathrm{C}_{12} \mathrm{H}_{13} \mathrm{FO}_{2}+\mathrm{Na}\right]^{+} 231.0792$ found 231.0791.

\section{Gram-Scale Synthesis of $16 \mathrm{~h}$}<smiles>Cc1ccc(C(=Cc2ccccc2)c2ccc(C)cc2)cc1</smiles>

$15 \mathrm{~h}$

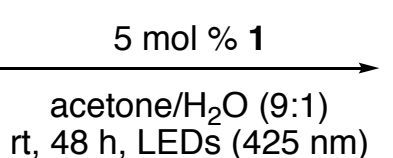

rt, $48 \mathrm{~h}$, LEDs $(425 \mathrm{~nm})$<smiles>Cc1ccc(C(O)(CCF)c2ccc(C)cc2)cc1</smiles>

$16 \mathrm{~h}$

$1.30 \mathrm{~g}, 80 \%$

A $200 \mathrm{~mL}$ Schlenk tube containing 1,1-di-p-tolylethylene 15h (1.30 g, $6.25 \mathrm{mmol}), 3$ (3.07 g, $9.37 \mathrm{mmol})$ and 1,4-bis(diphenylamino)naphthalene $1(0.144 \mathrm{~g}, 0.312 \mathrm{mmol})$ in acetone $(112.5 \mathrm{~mL})$ and $\mathrm{H}_{2} \mathrm{O}(12.5 \mathrm{~mL})$ was carefully degassed by three freeze-pump-thaw cycles, and placed at 2-3 cm away from blue LED lamps $(\lambda=425 \mathrm{~nm})$ in a water bath. The mixture was stirred for $48 \mathrm{~h}$ at room temperature under visible light irradiation. After the reaction, the reaction mixture was concentrated under reduced pressure. Then, the residue was dissolved in $\mathrm{CH}_{2} \mathrm{Cl}_{2}$ and washed with water. The water phase was extracted with $\mathrm{CH}_{2} \mathrm{Cl}_{2}$, and the combined organic layer was dried over $\mathrm{Na}_{2} \mathrm{SO}_{4}$, filtered and evaporated. $16 \mathrm{~h}$ was obtained as a colorless oil ( $1.30 \mathrm{~g}, 5.02 \mathrm{mmol}, 80 \%$ ) after purification by column chromatography on silica-gel (hexane:ethyl acetate $=10: 1$ ) and recycling preparative HPLC. 


\section{Photophysical and Electrochemical Measurements}

UV-vis spectra of 1 in acetone

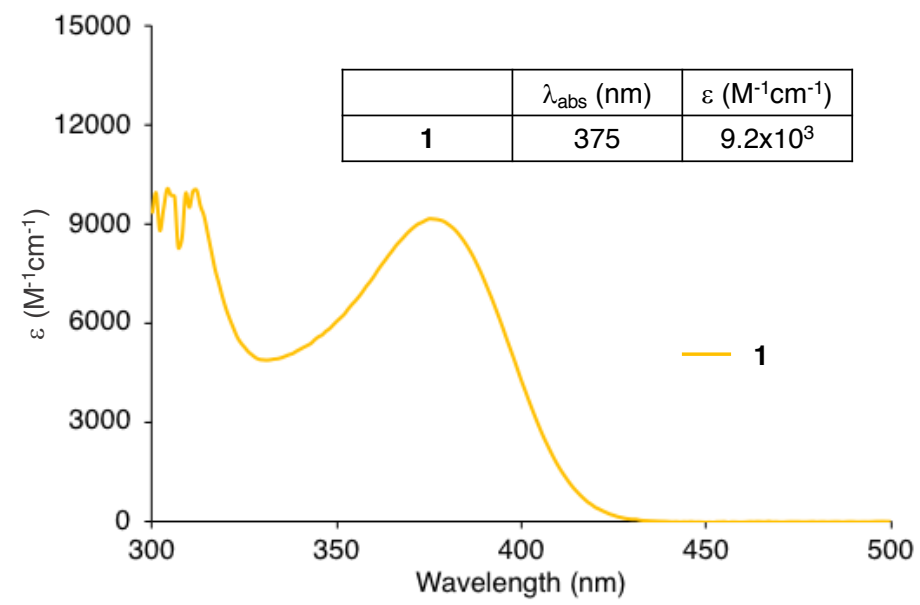

Figure S8. UV-vis spectra of 1. The measurements were performed in acetone $\left([1]=1.0 \times 10^{-4} \mathrm{M}\right)$ under air at room temperature.

Luminescence spectra and quantum yield of emission for 1 in acetone at room temperature
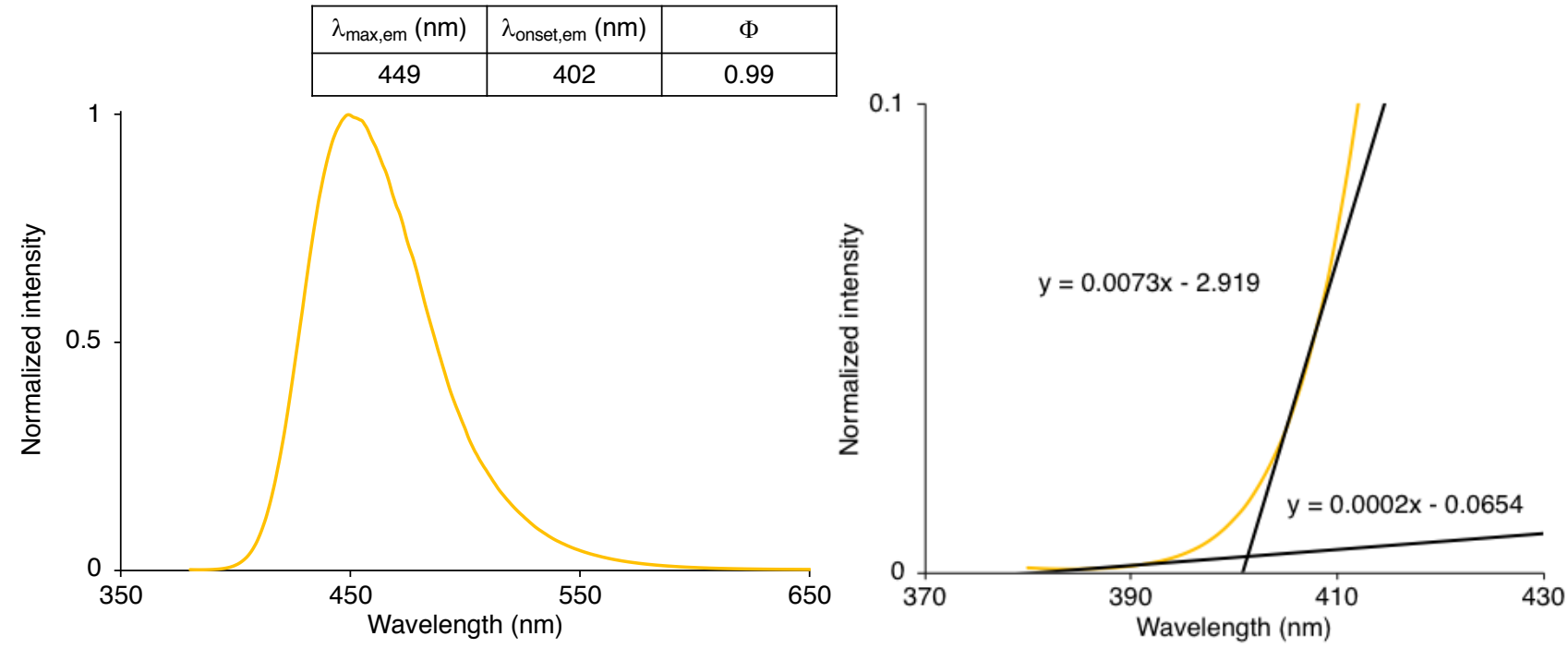

Figure S9. Luminescence spectrum of 1. The luminescence measurement (excited at $370 \mathrm{~nm}$ ) was performed in acetone $\left([1]=1.0 \times 10^{-4} \mathrm{M}\right)$ under $\mathrm{N}_{2}$ at room temperature, and the quantum yield measurement (excited at $370 \mathrm{~nm}$ ) was performed in acetone $\left([1]=1.0 \times 10^{-5} \mathrm{M}\right.$ ) under $\mathrm{N}_{2}$ at room temperature. 
UV-vis spectra of 1 in various solvents

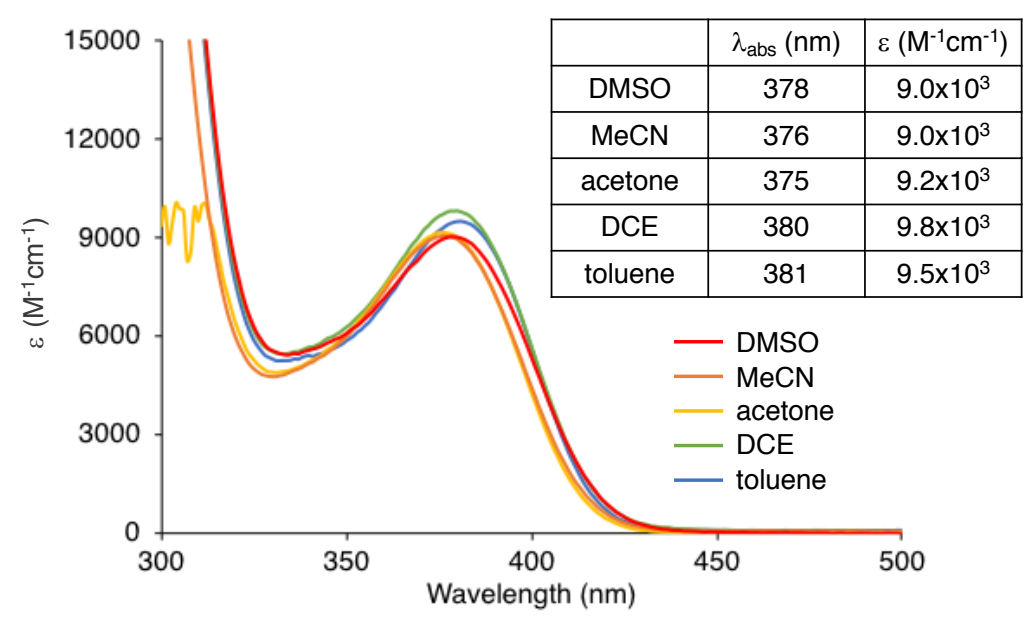

Figure S10. UV-vis spectra of 1 . The measurements were performed in toluene, 1,2-dichloroethane, acetone, acetonitrile and dimethyl sulfoxide $\left([1]=1.0 \times 10^{-4} \mathrm{M}\right)$ under air at room temperature.

Luminescence spectra and quantum yields of emission for 1 in various solvents at room temperature

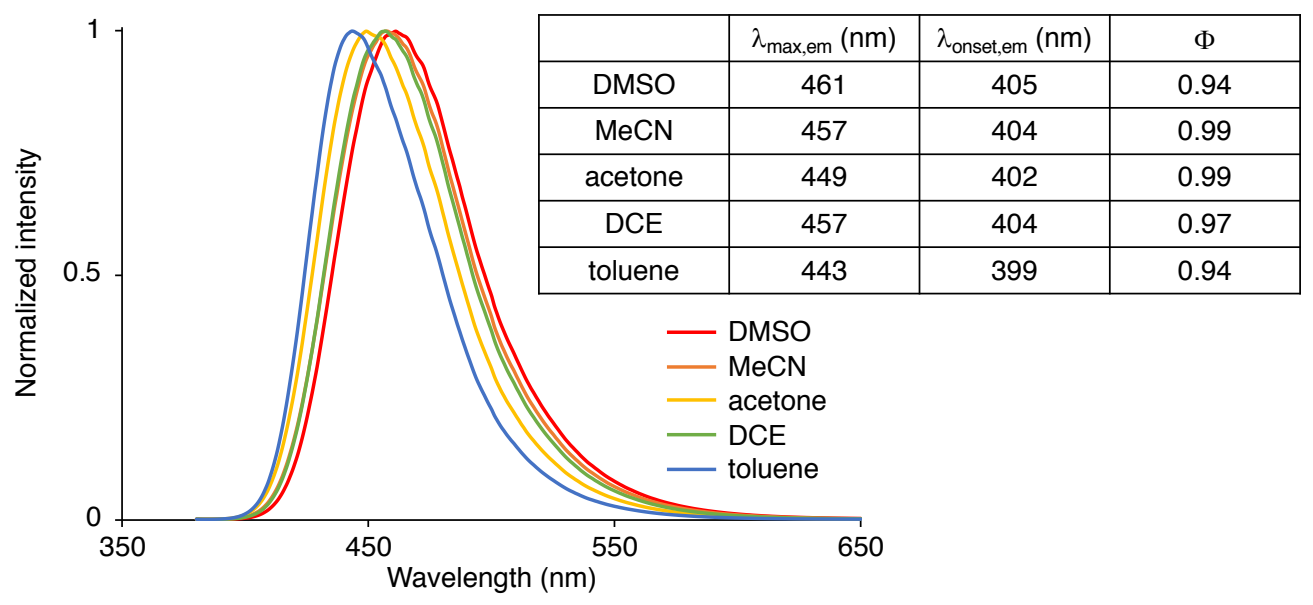

Figure S11. Luminescence spectra of 1. The luminescence measurements (excited at $370 \mathrm{~nm}$ ) were performed in toluene, 1,2-dichloroethane, acetonitrile and dimethyl sulfoxide ([1] = $1.0 \times 10^{-4} \mathrm{M}$ ) under $\mathrm{N}_{2}$ at room temperature, and the quantum yield measurements (excited at $370 \mathrm{~nm}$ ) were performed in acetone $\left([1]=1.0 \times 10^{-5} \mathrm{M}\right)$ under $\mathrm{N}_{2}$ at room temperature . 
Cyclic voltammograms of 1 in acetone at room temperature

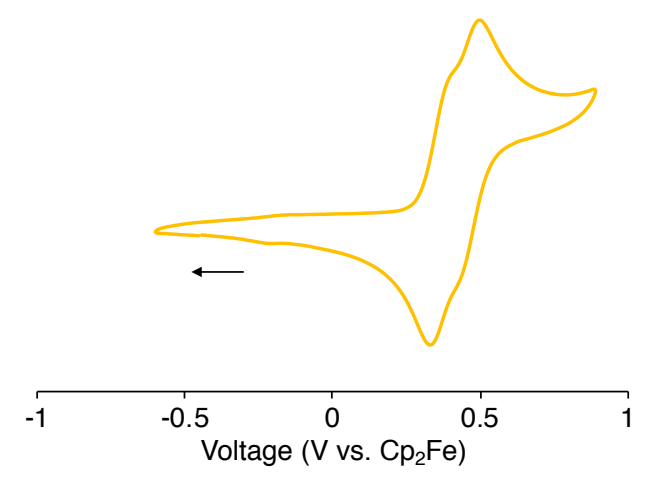

Figure S12. Cyclic voltammograms of 1. The measurements were performed in acetone $([1]=1.0 \mathrm{mM}$, $\left[\left(\mathrm{NBu}_{4}\right) \mathrm{PF}_{6}\right]=0.10 \mathrm{M}$ ) with platinum disk (working electrode) and wire electrodes (counter electrode), and a $\mathrm{Ag} / \mathrm{AgNO}_{3}$ reference electrode. The scan rates were $100 \mathrm{mV} / \mathrm{s}$. Ferrocene was used as a reference.

Differential pulse voltammograms of 1 in acetone at room temperature

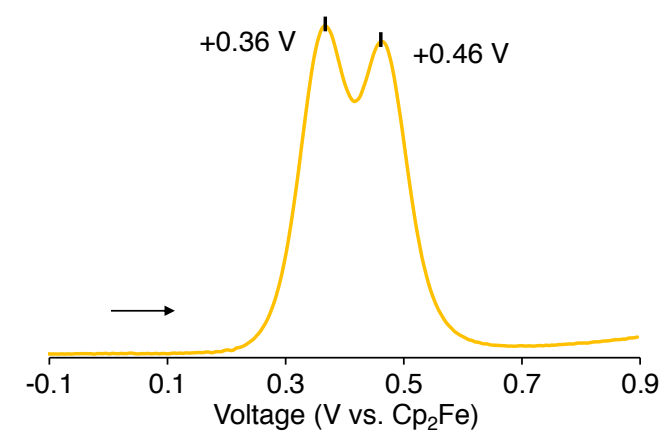

Figure S13. Differential pulse voltammograms of 1 . The measurements were performed in acetone ([1] $=$ $1.0 \mathrm{mM},\left[\left(\mathrm{NBu}_{4}\right) \mathrm{PF}_{6}\right]=0.10 \mathrm{M}$ ) with platinum disk (working electrode) and wire electrodes (counter electrode), and a $\mathrm{Ag} / \mathrm{AgNO}_{3}$ reference electrode. Ferrocene was used as a reference. 
Cyclic voltammograms of 1 in various solvents at room temperature
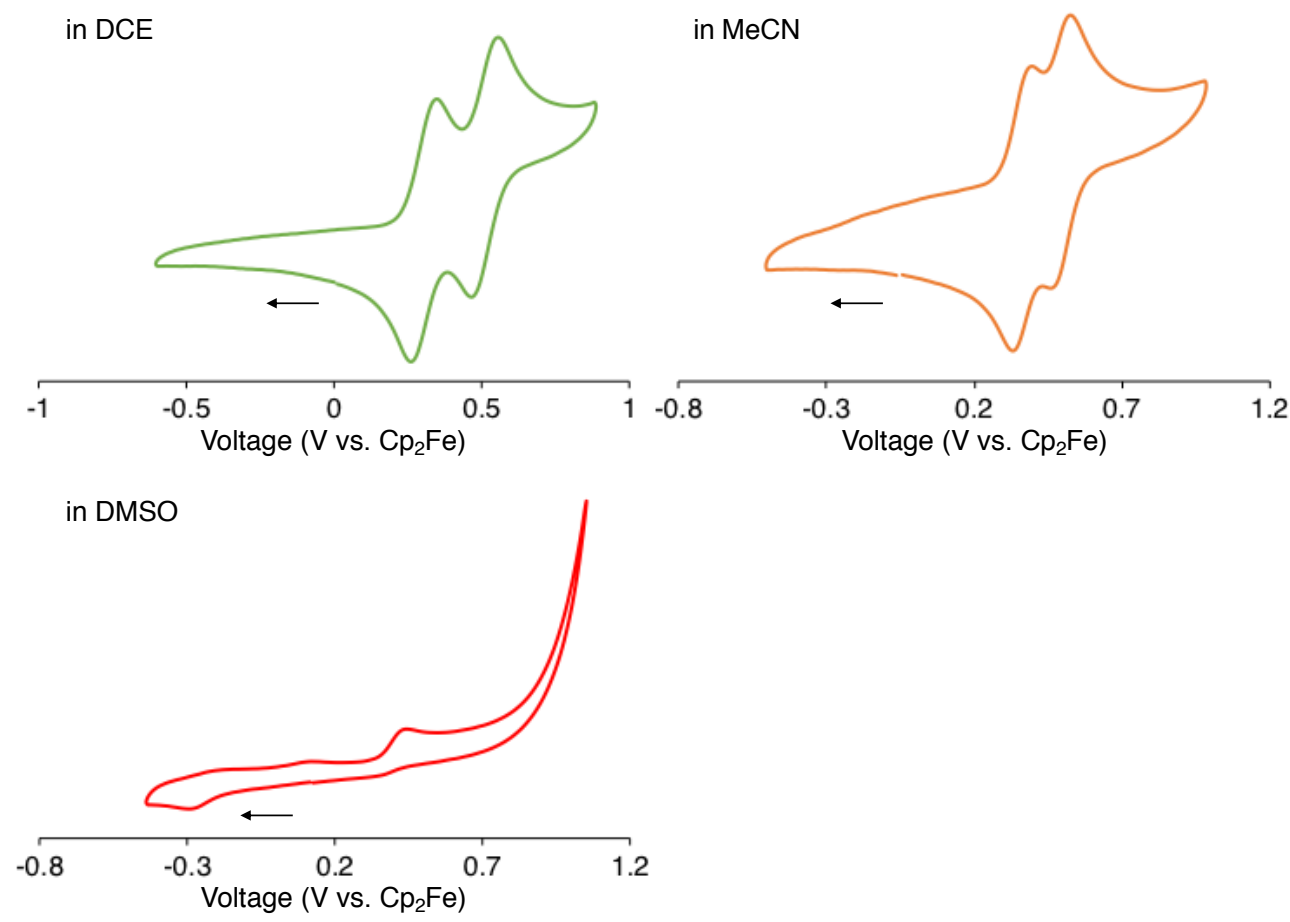

Figure S14. Cyclic voltammograms of 1. The measurements were performed in 1,2-dichloroethane, acetonitrile and dimethyl sulfoxide $\left([1]=1.0 \mathrm{mM},\left[\left(\mathrm{NBu}_{4}\right) \mathrm{PF}_{6}\right]=0.10 \mathrm{M}\right.$ ) with platinum disk (working electrode) and wire electrodes (counter electrode), and a $\mathrm{Ag} / \mathrm{AgNO}_{3}$ reference electrode. The scan rates were $100 \mathrm{mV} / \mathrm{s}$. Ferrocene was used as a reference. 
Differential pulse voltammograms of 1 in various solvents at room temperature
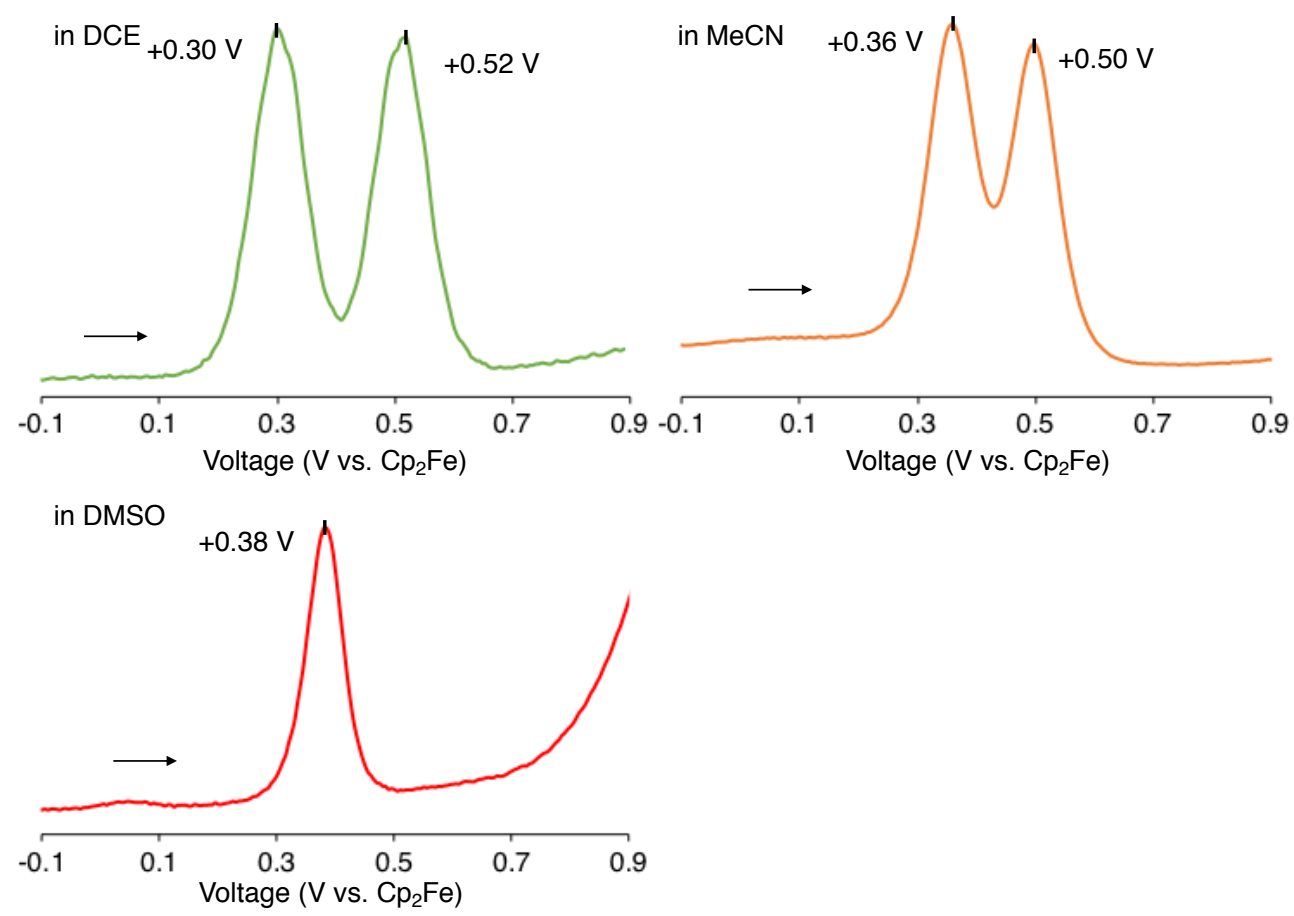

Figure S15. Differential pulse voltammograms of 1 . The measurements were performed in 1,2dichloroethane, acetonitrile and dimethyl sulfoxide $\left([1]=1.0 \mathrm{mM},\left[\left(\mathrm{NBu}_{4}\right) \mathrm{PF} 6\right]=0.100 \mathrm{M}\right)$ with platinum disk (working electrode) and wire electrodes (counter electrode), and a $\mathrm{Ag} / \mathrm{AgNO}_{3}$ reference electrode. Ferrocene was used as a reference.

Table S1. Estimated $E_{0,0}$ and $E^{*}$ ox of 1.

\begin{tabular}{cccc}
\hline & $E_{0,0}(\mathrm{eV})^{\mathrm{a}}\left(\right.$ from $\left.\lambda_{\max }\right)$ & $E_{0,0}(\mathrm{eV})^{\mathrm{a}}$ (from $\left.\lambda_{\text {onset }}\right)$ & $E_{\text {ox }}^{*}(\mathrm{~V} \text { vs. } \mathrm{Cp} 2 \mathrm{Fe})^{\mathrm{b}}$ \\
\hline $\mathbf{1}$ in acetone & 2.76 & 3.08 & $-2.72 \sim-2.40$ \\
$\mathbf{1}$ in 1,2-dichloroethane & 2.71 & 3.07 & $-2.77 \sim-2.41$ \\
$\mathbf{1}$ in acetonitrile & 2.71 & 3.07 & $-2.71 \sim-2.35$ \\
$\mathbf{1}$ in dimethyl sulfoxide & 2.69 & 3.06 & $-2.68 \sim-2.31$ \\
\hline
\end{tabular}

${ }^{a} E_{0,0}$ was estimated from $\lambda_{\text {max,em. }}$

${ }^{\mathrm{b}} E_{\mathrm{ox}}$ was estimated from DPV, and $E^{*}$ ox were estimated from a following expression.

$E^{*}{ }_{\mathrm{ox}}=E_{\mathrm{ox}}-E_{0,0}$ 
Cyclic voltammograms of $\mathbf{3}$ in various solvents at room temperature

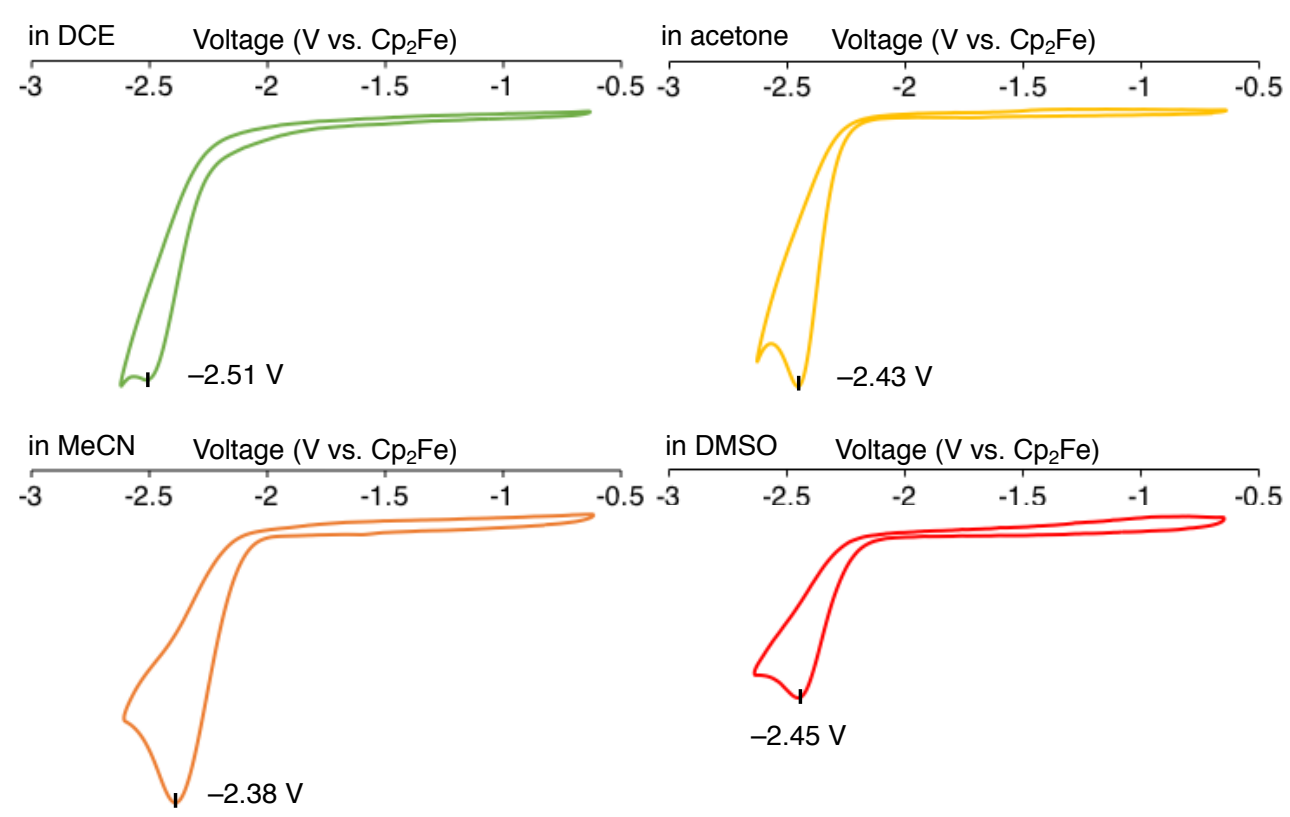

Figure S16. Cyclic voltammograms of 3. The measurements were performed in 1,2-dichloroethane, acetone, acetonitrile and dimethyl sulfoxide $\left([3]=5.0 \mathrm{mM},\left[\left(\mathrm{NBu}_{4}\right) \mathrm{PF}_{6}\right]=0.10 \mathrm{M}\right)$ with platinum disk (working electrode) and wire electrodes (counter electrode), and a $\mathrm{Ag} / \mathrm{AgNO}_{3}$ reference electrode. The scan rates were $100 \mathrm{mV} / \mathrm{s}$. Ferrocene was used as a reference.

Cyclic voltammograms of $\mathbf{5}$ and $\mathbf{8}$ in acetone at room temperature
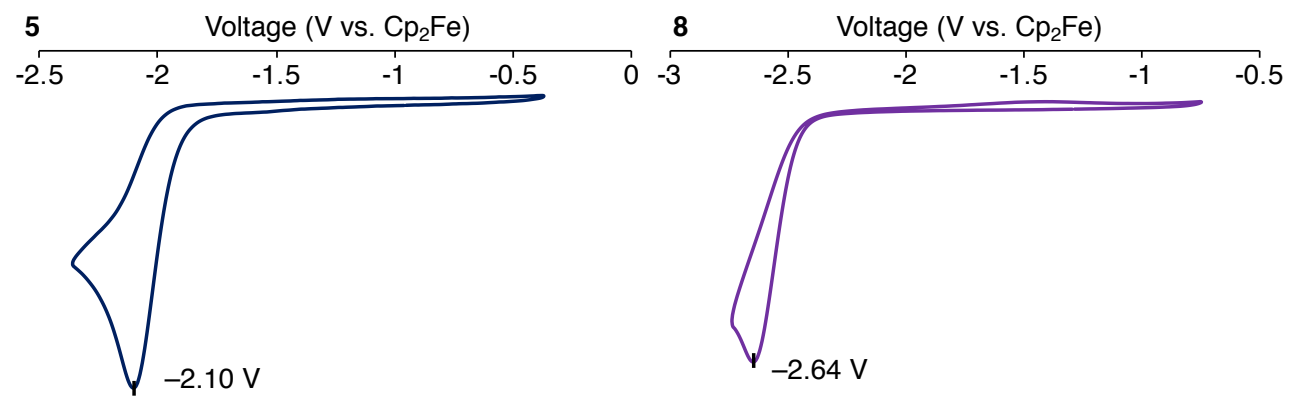

Figure S17. Cyclic voltammograms of 5 and $\mathbf{8}$. The measurements were performed in 1,2-dichloroethane, acetone, acetonitrile and dimethyl sulfoxide $\left([5],[8]=5.0 \mathrm{mM},\left[\left(\mathrm{NBu}_{4}\right) \mathrm{PF}_{6}\right]=0.10 \mathrm{M}\right)$ with platinum disk (working electrode) and wire electrodes (counter electrode), and a $\mathrm{Ag} / \mathrm{AgNO}_{3}$ reference electrode. The scan rates were $100 \mathrm{mV} / \mathrm{s}$. Ferrocene was used as a reference. 
Excited state lifetime of 1 in various solvents at room temperature
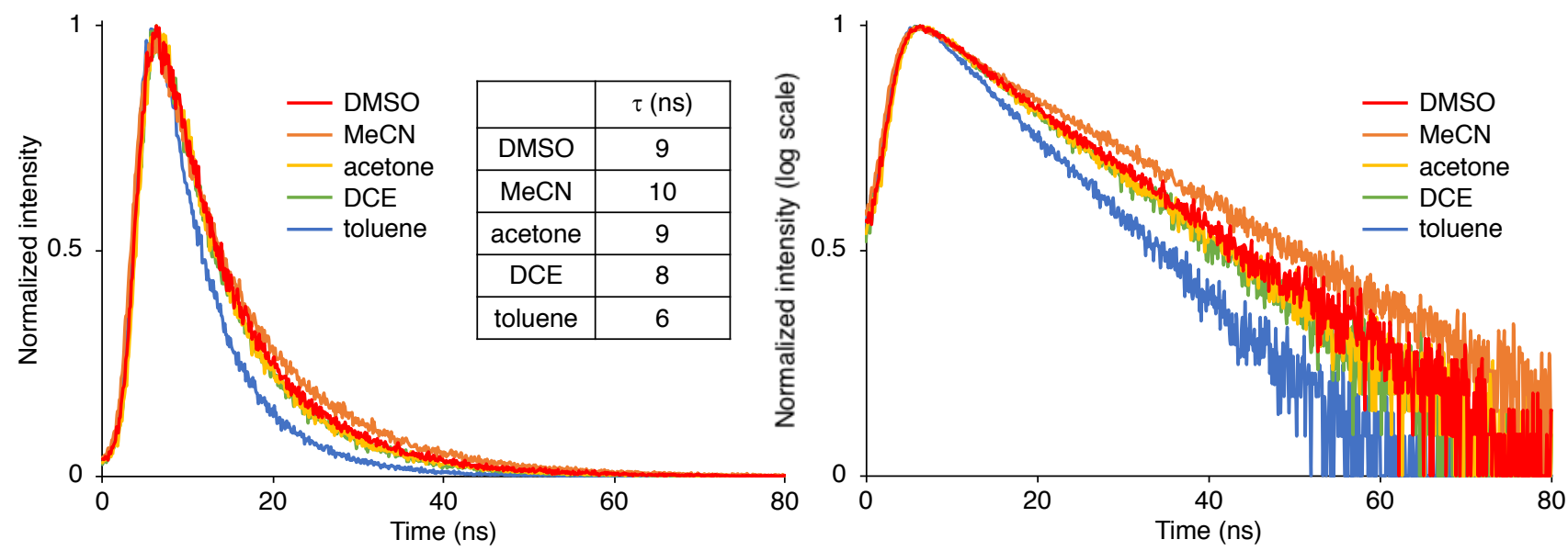

Figure S18. Luminescence decay of 1. The measurements were performed in toluene, 1,2-dichloroethane, acetone, acetonitrile and dimethyl sulfoxide $\left([1]=1.0 \times 10^{-4} \mathrm{M}\right.$, excitation light: $\left.\lambda=355 \mathrm{~nm}\right)$ under $\mathrm{N}_{2}$ at room temperature.

Luminescence spectrum of $f a c-\left[\operatorname{lr}(\mathrm{ppy})_{3}\right] 9$ in acetone at room temperature

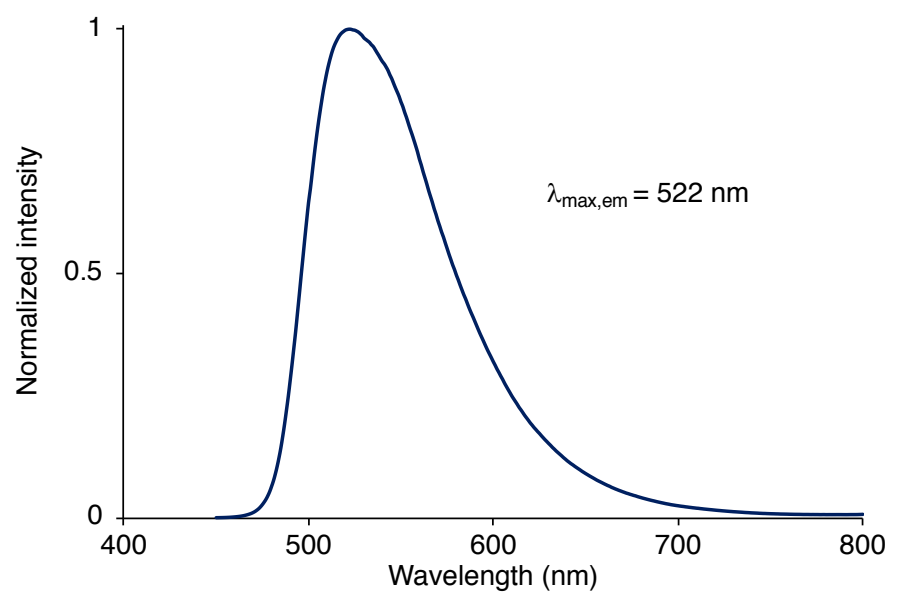

Figure S19. Luminescence spectrum of 9. The luminescence measurement was performed in acetone ([9] $=10^{-4} \mathrm{M}$, excitation light: $\lambda=410 \mathrm{~nm}$ ) under $\mathrm{N}_{2}$ atmosphere at room temperature. 
Cyclic voltammogram of $\mathrm{fac}-\left[\mathrm{Ir}(\mathrm{ppy})_{3}\right] \mathbf{9}$ in acetone at room temperature

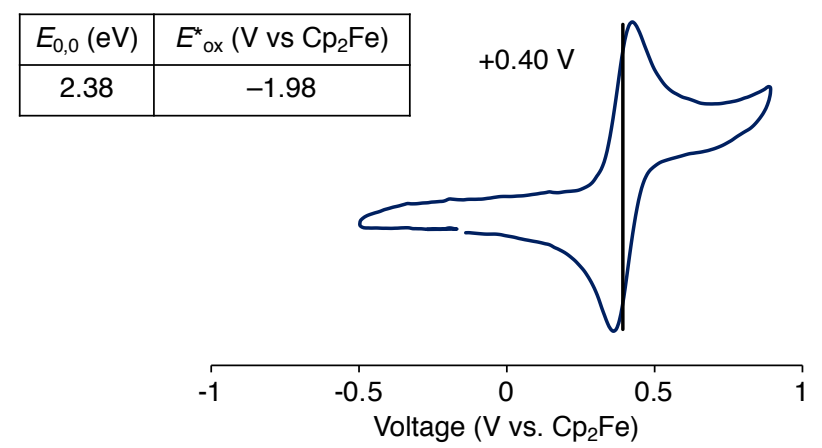

Figure S20. Cyclic voltammogram of 9. The measurement was performed in acetone $([9]=1.0 \mathrm{mM}$, $\left[\left(\mathrm{NBu}_{4}\right) \mathrm{PF}_{6}\right]=0.10 \mathrm{M}$ ) with platinum disk (working electrode) and wire electrodes (counter electrode), and a $\mathrm{Ag} / \mathrm{AgNO}_{3}$ reference electrode. The scan rate was $100 \mathrm{mV} / \mathrm{s}$. Ferrocene was used as a reference. 


\section{Luminescence Quenching Experiments}

Luminescence quenching experiments of 1 were performed in acetone $\left([1]=2.5 \times 10^{-3} \mathrm{M}\right)$ at room temperature. The solutions were degassed by three freeze-pump-thaw cycles before the measurements.

Changes of luminescence spectra
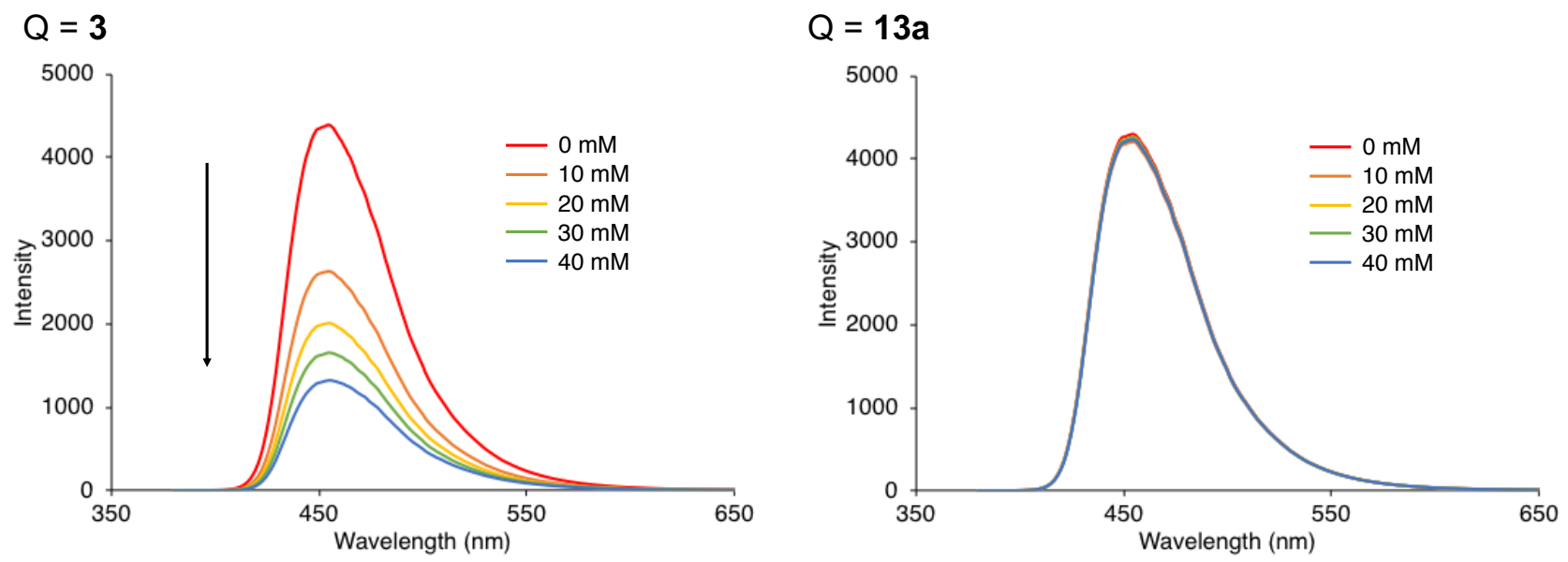

Figure S21. Changes of luminescence spectra of 1.

Stern-Volmer plots

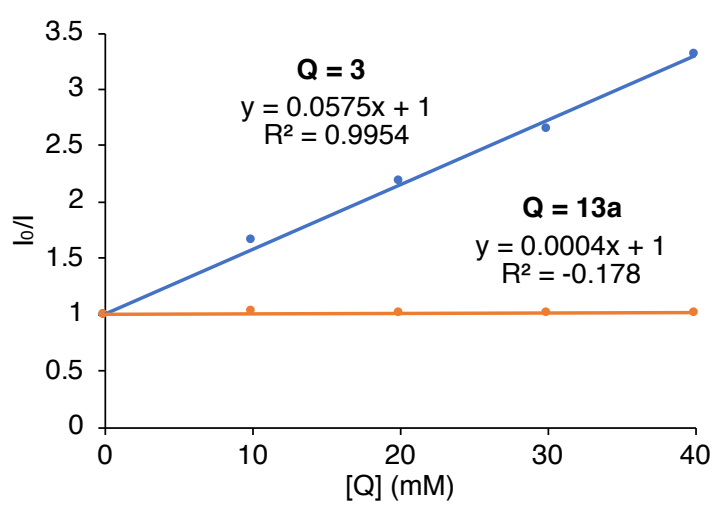

Figure S22. Stern-Volmer plots. 
Comparison of luminescence spectra

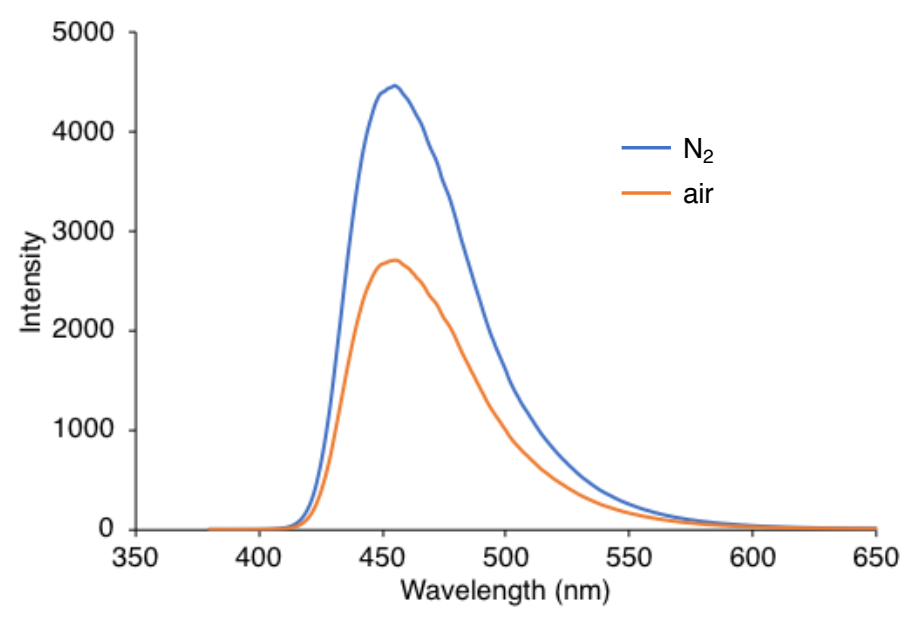

Figure S23. Comparison of luminescence spectra of 1 under air $/ \mathrm{N}_{2}$. The luminescence measurement was performed in acetone $\left([9]=2.5 \times 10^{-3} \mathrm{M}\right.$, excitation light: $\left.\lambda=370 \mathrm{~nm}\right)$ at room temperature. 


\section{Light/Dark Experiments}
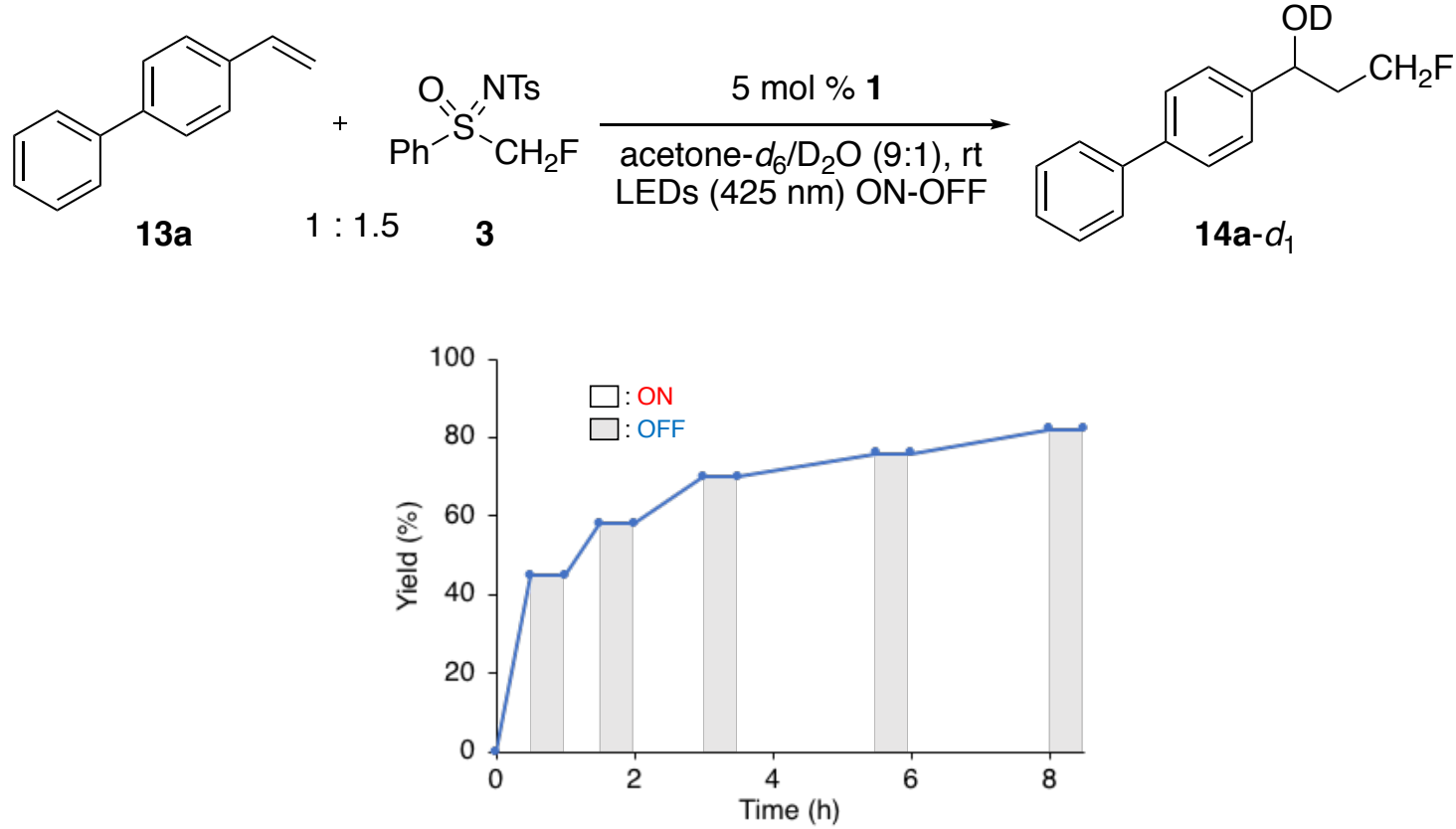

Figure S24. Time profile for the reaction under light and dark conditions. 


\section{Quantum Yield Measurement of the Reaction with 13a}

(Photon flux)

Photon flux was measured with Ophir PD300-UV and Ophir StarLite.

Irradiation was carried out with HITACHI F-7000 $(\lambda=410 \mathrm{~nm}$, emission slit width $=10.0 \mathrm{~nm})$.

Measured power of light: $121.4 \mu \mathrm{W}$ (for $\lambda=410 \mathrm{~nm}$ )

$$
\begin{aligned}
\text { photon flux }= & \frac{\text { power of light }(\mathrm{W}) \times \text { wavelength of irradiation light }(\mathrm{m})}{\text { Plank constant }(\mathrm{J} \cdot \mathrm{s}) \times \text { speed of light }(\mathrm{m} / \mathrm{s}) \times \text { Avogadro constant }(1 / \mathrm{mol})} \\
& \therefore \frac{121.4 \times 10^{-6} \times 410 \times 10^{-9}}{6.626 \times 10^{-34} \times 2.998 \times 10^{8} \times 6.022 \times 10^{23}}=4.16 \times 10^{-10}
\end{aligned}
$$

Calculated photon flux: $4.16 \times 10^{-10}$ einstein $\bullet \mathrm{s}^{-1}$

(Quantum yield)

A cuvette was charged with a solution of $p$-vinylbiphenyl 13a $(18.0 \mathrm{mg}, 0.100 \mathrm{mmol}), 3(12.3 \mathrm{mg}, 37.6$ $\mu \mathrm{mol}), 1(2.3 \mathrm{mg}, 1.25 \mu \mathrm{mol})$, acetone $(1.8 \mathrm{~mL})$ and $\mathrm{H}_{2} \mathrm{O}(0.2 \mathrm{~mL})$. The solution was degassed by three freeze-pump-thaw cycles, and it was irradiated by F-7000 $(\lambda=410 \mathrm{~nm}$, emission slit width $=10.0 \mathrm{~nm})$ for $24 \mathrm{~h}$. After evaporation, the yield of 14a was determined by ${ }^{1} \mathrm{H}$ NMR with dimethylsulfone $(4.71 \mathrm{mg}, 50.0$ $\mathrm{mmol}$ ) as an internal standard.

The quantum yield $(\Phi)$ was calculated by following formula. (A: Absorbance of catalysts)

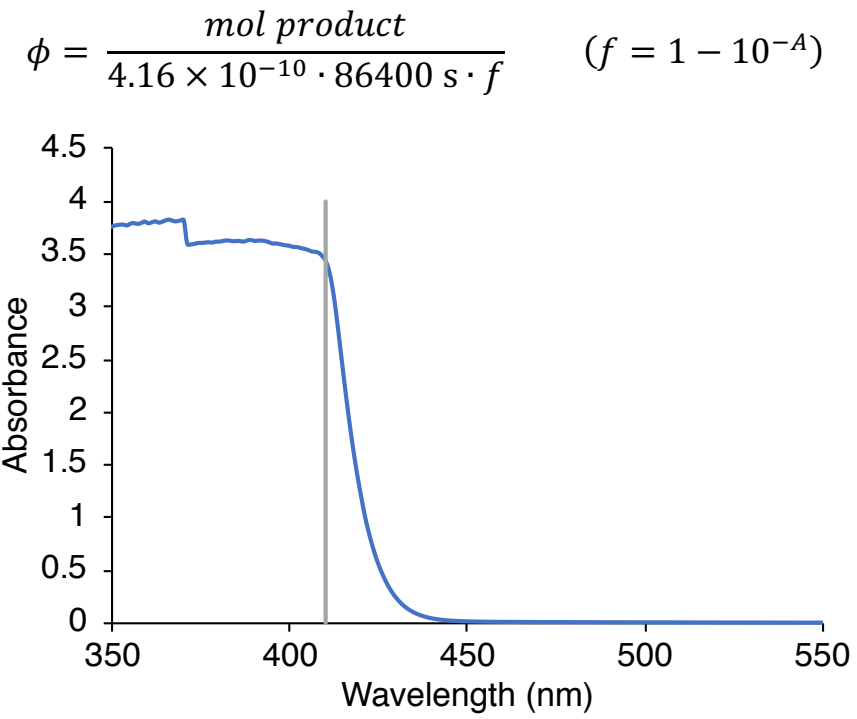

Figure S25. UV-vis spectra of $1(A=3.43716, f=0.9996)$.

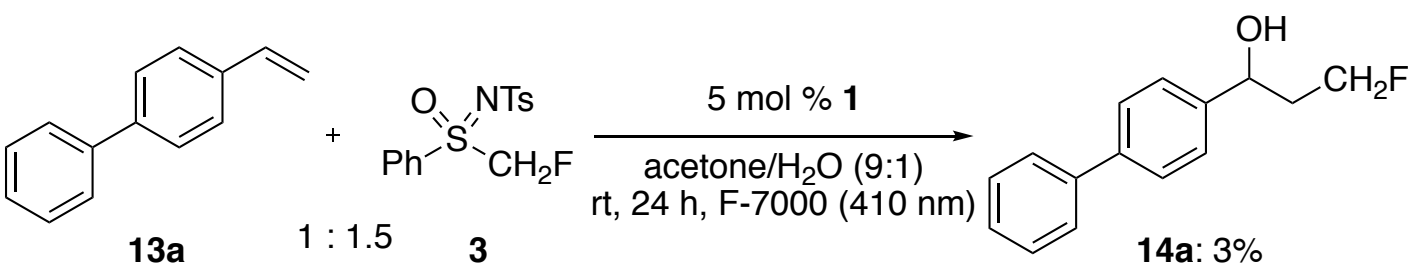

Yield of 14a: $3 \%$ (mol product $\left.=3 \times 10^{-6} \mathrm{~mol}\right)$, Calculated quantum yield: $\Phi=\mathbf{8} \%$ 


\section{DFT and TD-DFT Calculation of 1}

Theoretical calculations were performed by using Gaussian 09(D01) and 16 (A03) program package. Ground state geometries of 1 were obtained with the CAM-B3LYP/6-31G(d)/CPCM-acetone level of theory. Single point TD-DFT calculations were performed with the CAM-B3LYP/6-31G(d)/CPCM-acetone level of theory. The first three excited states of 1 are shown below.

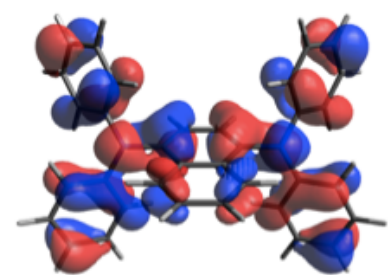

HOMO (122)

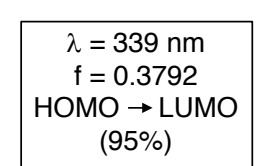

$(95 \%)$

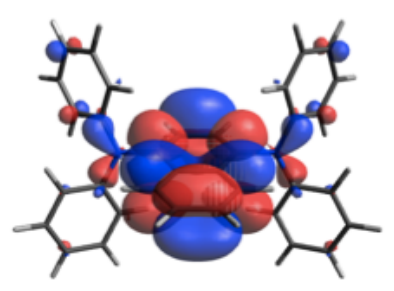

LUMO (123)

Figure S26. Calculated molecular orbitals of 1.

Excited states of 1

Excited State 1: Singlet-A $3.6594 \mathrm{eV} \quad 338.81 \mathrm{n} \quad \mathrm{f}=0.3792 \quad\left\langle\mathrm{~S}^{* *} 2>=0.000\right.$

$122->123 \quad 0.68871\left(2 \times 0.68871^{2} \times 100=95 \%\right.$ contribution $)$

Excited State 2: Singlet-A $\quad 4.2027 \mathrm{eV} \quad 295.01 \mathrm{~nm} \quad \mathrm{f}=0.0029 \quad\left\langle\mathrm{~S}^{* *} 2>=0.000\right.$

$$
\begin{array}{ll}
119->123 & -0.10781 \\
121->123 & 0.65906 \\
122->124 & -0.14057
\end{array}
$$

Excited State 3: Singlet-A $4.3389 \mathrm{eV} \quad 285.75 \mathrm{~nm} \quad \mathrm{f}=0.0901 \quad\left\langle\mathrm{~S}^{* *} 2>=0.000\right.$

$$
\begin{array}{ll}
119->123 & 0.23083 \\
120->124 & -0.14103 \\
120->128 & 0.10649 \\
121->123 & 0.17995 \\
121->127 & 0.10570 \\
122->124 & 0.57531
\end{array}
$$

\begin{tabular}{|c|c|c|}
\hline & Measured $\lambda_{\mathrm{ab}}$ & Calculated $\lambda_{\mathrm{ab}}$ \\
\hline $\mathbf{1}$ & 375 & $339(\mathrm{H}-\mathrm{-} \mathrm{L})$ \\
\hline
\end{tabular}

Since the maximum absorptions were assigned as electron transition from HOMO to LUMO in both cases of 1 , the major visible light absorptions were assigned to excitation from whole the molecules to the central naphthalene rings. 
Table S2. Cartesian coordinates of 1 (-1419.97708462 hartree, neutral, singlet).

\begin{tabular}{|c|c|c|c|}
\hline C & 0.70568 & -1.7063 & 3.06579 \\
\hline C & 1.39563 & -1.11767 & 2.03949 \\
\hline C & 0.7122 & -0.50496 & 0.95802 \\
\hline C & -0.71227 & -0.50497 & 0.95796 \\
\hline C & -1.3958 & -1.11775 & 2.03932 \\
\hline C & -0.70595 & -1.70636 & 3.0657 \\
\hline $\mathrm{H}$ & 1.24453 & -2.16569 & 3.88837 \\
\hline $\mathrm{H}$ & 2.47882 & -1.10375 & 2.04744 \\
\hline C & 1.40985 & 0.14595 & -0.10737 \\
\hline C & -1.40987 & 0.14593 & -0.10747 \\
\hline $\mathrm{H}$ & -2.479 & -1.10391 & 2.04712 \\
\hline$\pi$ & -1.24488 & -2.16578 & 3.88821 \\
\hline C & -0.70376 & 0.79053 & -1.09178 \\
\hline C & 0.70381 & 0.79054 & -1.09173 \\
\hline $\mathrm{H}$ & -1.24098 & 1.2898 & -1.89121 \\
\hline$H$ & 1.24108 & 1.2898 & -1.89112 \\
\hline$N$ & -2.8355 & 0.14691 & -0.13215 \\
\hline $\mathrm{N}$ & 2.83549 & 0.14691 & -0.13195 \\
\hline C & -3.50338 & 1.39733 & -0.15743 \\
\hline C & -4.5599 & 1.64398 & -1.03892 \\
\hline C & -3.08738 & 2.41758 & 0.70384 \\
\hline C & -5.19239 & 2.8815 & -1.04474 \\
\hline $\mathrm{H}$ & -4.88443 & 0.86496 & -1.71969 \\
\hline C & -3.71315 & 3.65686 & 0.67894 \\
\hline $\mathrm{H}$ & -2.27069 & 2.23116 & 1.39326 \\
\hline C & -4.773 & 3.89649 & -0.19087 \\
\hline $\mathrm{H}$ & -6.01176 & 3.05492 & -1.73575 \\
\hline $\mathrm{H}$ & -3.37567 & 4.43672 & 1.3548 \\
\hline $\mathrm{H}$ & -5.26563 & 4.86327 & -0.2033 \\
\hline C & -3.52339 & -1.0624 & -0.38457 \\
\hline C & -2.91443 & -2.09342 & -1.1059 \\
\hline C & -4.81482 & -1.26347 & 0.11966 \\
\hline C & -3.58201 & -3.29519 & -1.31444 \\
\hline $\mathrm{H}$ & -1.91646 & -1.9515 & -1.50522 \\
\hline 0 & -5.47898 & -2.4604 & -0.10854 \\
\hline F & -5.29392 & -0.47751 & 0.69301 \\
\hline & -4.86825 & -3.48737 & -0.82386 \\
\hline
\end{tabular}




\begin{tabular}{|c|c|c|c|}
\hline $\mathrm{H}$ & -3.08984 & -4.08305 & -1.87667 \\
\hline $\mathrm{H}$ & -6.48003 & -2.59494 & 0.29 \\
\hline $\mathrm{H}$ & -5.38837 & -4.42441 & -0.99349 \\
\hline C & 3.5234 & -1.0624 & -0.38433 \\
\hline C & 2.91444 & -2.09349 & -1.10558 \\
\hline C & 4.81484 & -1.26346 & 0.11991 \\
\hline C & 3.582 & -3.29529 & -1.31401 \\
\hline $\mathrm{H}$ & 1.91648 & -1.9516 & -1.50495 \\
\hline C & 5.47897 & -2.46043 & -0.1082 \\
\hline $\mathrm{H}$ & 5.29398 & -0.47749 & 0.69318 \\
\hline C & 4.86824 & -3.48746 & -0.82342 \\
\hline $\mathrm{H}$ & 3.0898 & -4.08317 & -1.87619 \\
\hline $\mathrm{H}$ & 6.48002 & -2.59492 & 0.29035 \\
\hline $\mathrm{H}$ & 5.38835 & -4.42452 & -0.99297 \\
\hline C & 3.5034 & 1.39731 & -0.15753 \\
\hline C & 4.55986 & 1.64379 & -1.03912 \\
\hline C & 3.08753 & 2.41772 & 0.70364 \\
\hline C & 5.1924 & 2.88129 & -1.04518 \\
\hline $\mathrm{H}$ & 4.88432 & 0.86466 & -1.71982 \\
\hline C & 3.71335 & 3.65695 & 0.67851 \\
\hline $\mathrm{H}$ & 2.27089 & 2.23143 & 1.39316 \\
\hline C & 4.77315 & 3.89642 & -0.19142 \\
\hline $\mathrm{H}$ & 6.01174 & 3.05455 & -1.73627 \\
\hline $\mathrm{H}$ & 3.37597 & 4.43692 & 1.3543 \\
\hline $\mathrm{H}$ & 5.26584 & 4.86316 & -0.20402 \\
\hline
\end{tabular}




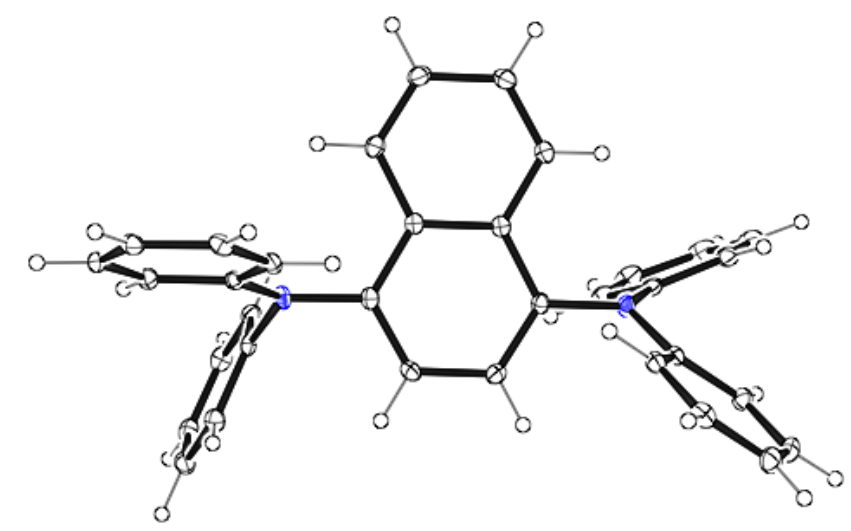

Figure S27. An ORTEP drawing of 1.

Table S3-1. Crystal data and structure refinement for 1.

Chemical formula
Formula weight
Temperature
Wavelength
Crystal size
Crystal system
Space group
Unit cell dimensions

Volume

Z

Density (calculated)

Absorption coefficient

$F(000)$

Theta range for data collection

Index ranges

Reflections collected

Independent reflections

Coverage of independent reflections

Absorption correction

Max. and min. transmission

Refinement method
$\mathrm{C}_{34} \mathrm{H}_{26} \mathrm{~N}_{2}$

462.57

90(2) K

$0.71073 \AA$

$0.034 \times 0.089 \times 0.446 \mathrm{~mm}$

monoclinic

P $121 /$ n 1

$a=11.5593(11) \AA$

$\alpha=90^{\circ}$

$\mathrm{b}=7.8828(7) \AA$

$\beta=98.0430(10)^{\circ}$

$\mathrm{c}=27.350(3) \AA$

$Y=90^{\circ}$

2467.6(4) $\AA^{3}$

4

$1.245 \mathrm{~g} / \mathrm{cm}^{3}$

$0.072 \mathrm{~mm}^{-1}$

976

1.50 to $25.02^{\circ}$

$-13<=\mathrm{h}<=12,-9<=\mathrm{k}<=9,-26<=\mathrm{l}<=32$

11428

$4362[R($ int $)=0.0183]$

$99.70 \%$

multi-scan

0.9980 and 0.9680

Full-matrix least-squares on $\mathrm{F}^{2}$ 


\section{Refinement program}

Function minimized

Data / restraints / parameters

Goodness-of-fit on $\mathrm{F}^{2}$

$\Delta / \sigma_{\max }$

Final $\mathbf{R}$ indices

Weighting scheme

Extinction coefficient

Largest diff. peak and hole

R.M.S. deviation from mean
SHELXL-2013 (Sheldrick, 2013)

$\Sigma w\left(F_{o}^{2}-F_{c}^{2}\right)^{2}$

4362 / 0 / 326

1.006

0.001

3659 data; $\mid>2 \sigma(I)$

$\mathrm{R} 1=0.0319, \mathrm{wR} 2=0.0730$

all data

$\mathrm{R} 1=0.0408, \mathrm{wR} 2=0.0773$

$w=1 /\left[\sigma^{2}\left(F_{o}^{2}\right)+(0.0312 P)^{2}+0.9572 P\right]$

where $\mathrm{P}=\left(\mathrm{F}_{\mathrm{o}}{ }^{2}+2 \mathrm{~F}_{\mathrm{c}}{ }^{2}\right) / 3$

$0.0065(5)$

0.180 and -0.170 e $\AA^{-3}$

$0.034 \mathrm{e}^{-3}$

Table S3-2. Atomic Coordinates and Equivalent Isotropic Atomic Displacement Parameters (Å2) for 1.

$\mathbf{x} / \mathbf{a}$

C1

C2

C3

C4

C5

C6

C7

C8

C9

C10

C11

C12

C13

C14

C15

C16

C17

C18

C19

C20

C21 $y / b$

0.70088(10)

0.64290(11)

0.62545(11)

0.66584(10)

0.72399(9)

0.76486(10)

0.81987(11)

0.83899(11)

0.80178(10)

0.74254(10)

0.65689(10)

0.56610(10)

0.50168(11)

0.52566(11)

0.61560(10)

$0.68185(10)$

$0.77935(10)$

$0.71645(11)$

$0.77193(11)$

0.89037(11)

0.95287(11) z/c

0.98370(4)

0.95781(4)

0.90593(4)

0.88001(4)

0.90536(4)

$0.88008(4)$

0.90542(5)

0.95741(5)

0.98306(4)

0.95812(4)

0.06591(4)

$0.04483(4)$

0.07459(5)

$0.12557(5)$

$0.14663(4)$

$0.11747(4)$

$0.05604(4)$

$0.07545(4)$

$0.09335(4)$

0.09185(4)

$0.07215(5)$
$\mathrm{U}(\mathrm{eq})$

$0.0170(3)$

0.0214(3)

0.0216(3)

0.0167(3)

0.0156(3)

0.0184(3)

0.0229(3)

0.0237(3)

0.0198(3)

0.0158(3)

0.0168(3)

$0.0184(3)$

0.0207(3)

0.0209(3)

0.0197(3)

0.0190(3)

$0.0172(3)$

0.0210(3)

0.0233(3)

$0.0241(3)$

0.0246(3) 


$\begin{array}{lllll}\text { C22 } & 0.89784(10) & 0.05202(17) & 0.05429(4) & 0.0212(3) \\ \text { C23 } & 0.75668(10) & 0.91182(16) & 0.80598(4) & 0.0190(3) \\ \text { C24 } & 0.84794(11) & 0.00255(17) & 0.83240(5) & 0.0252(3) \\ \text { C25 } & 0.94801(12) & 0.0382(2) & 0.81126(6) & 0.0342(4) \\ \text { C26 } & 0.95678(12) & 0.9852(2) & 0.76372(6) & 0.0366(4) \\ \text { C27 } & 0.86707(12) & 0.89236(19) & 0.73794(5) & 0.0308(3) \\ \text { C28 } & 0.76808(12) & 0.85360(17) & 0.75871(5) & 0.0238(3) \\ \text { C29 } & 0.54116(10) & 0.90595(15) & 0.80058(4) & 0.0169(3) \\ \text { C30 } & 0.52467(11) & 0.00289(16) & 0.75764(4) & 0.0210(3) \\ \text { C31 } & 0.41323(11) & 0.03460(18) & 0.73364(5) & 0.0264(3) \\ \text { C32 } & 0.31592(11) & 0.97213(18) & 0.75195(5) & 0.0271(3) \\ \text { C33 } & 0.33168(11) & 0.87393(17) & 0.79427(5) & 0.0241(3) \\ \text { C34 } & 0.44267(11) & 0.84012(16) & 0.81834(4) & 0.0198(3) \\ \text { N1 } & 0.72385(9) & 0.87667(13) & 0.03659(3) & 0.0182(2) \\ \text { N2 } & 0.65358(8) & 0.87426(13) & 0.82712(3) & 0.0180(2)\end{array}$

Table S3-3. Bond Lengths $(\AA)$ for 1.

\begin{tabular}{|c|c|c|c|}
\hline C1-C2 & $1.3680(17)$ & C31-C32 & $1.3841(19)$ \\
\hline C1-N1 & $1.4352(15)$ & C32-C33 & $1.3832(19)$ \\
\hline C4-N2 & $1.4342(14)$ & C33-C34 & $1.3835(18)$ \\
\hline C5-C10 & $1.4288(16)$ & C1-C10 & $1.4264(17)$ \\
\hline C11-C16 & $1.4004(16)$ & C2-C3 & $1.4051(17)$ \\
\hline C12-C13 & $1.3873(17)$ & C3-C4 & $1.3676(17)$ \\
\hline C13-C14 & $1.3851(17)$ & C4-C5 & $1.4262(17)$ \\
\hline C14-C15 & $1.3845(18)$ & C5-C6 & $1.4172(17)$ \\
\hline C15-C16 & $1.3856(17)$ & $\mathrm{C} 6-\mathrm{C} 7$ & $1.3666(18)$ \\
\hline C17-C18 & $1.3900(17)$ & C7-C8 & $1.4082(18)$ \\
\hline C18-C19 & $1.3866(18)$ & C8-C9 & $1.3678(18)$ \\
\hline C19-C20 & $1.3854(18)$ & C9-C10 & $1.4173(17)$ \\
\hline C20-C21 & $1.3824(19)$ & C11-C12 & $1.3957(17)$ \\
\hline C21-C22 & $1.3892(19)$ & C11-N1 & $1.4092(15)$ \\
\hline C23-C28 & $1.3953(17)$ & C17-C22 & $1.3879(17)$ \\
\hline C24-C25 & $1.3923(19)$ & C17-N1 & $1.4363(16)$ \\
\hline C25-C26 & $1.383(2)$ & C23-C24 & $1.3905(18)$ \\
\hline C26-C27 & $1.379(2)$ & C23-N2 & $1.4265(15)$ \\
\hline C27-C28 & $1.3810(18)$ & C29-C30 & $1.3918(17)$ \\
\hline
\end{tabular}




$\begin{array}{llll}\mathrm{C} 29-\mathrm{C} 34 & 1.3985(17) & \mathrm{C} 29-\mathrm{N} 2 & 1.4191(15) \\ \mathrm{C} 30-\mathrm{C} 31 & 1.3842(18) & & \end{array}$

Table S3-4. Bond Angles ( ${ }^{\circ}$ ) for 1.

\begin{tabular}{cccc} 
C2-C1-C10 & $120.11(11)$ & C2-C1-N1 & $119.81(11)$ \\
C10-C1-N1 & $120.03(10)$ & C1-C2-C3 & $121.17(11)$ \\
C4-C3-C2 & $120.53(12)$ & C3-C4-C5 & $120.33(11)$ \\
C3-C4-N2 & $120.70(11)$ & C5-C4-N2 & $118.94(10)$ \\
C6-C5-C4 & $122.34(10)$ & C6-C5-C10 & $118.64(11)$ \\
C4-C5-C10 & $119.02(11)$ & C7-C6-C5 & $120.98(11)$ \\
C6-C7-C8 & $120.43(12)$ & C9-C8-C7 & $120.23(12)$ \\
C8-C9-C10 & $121.00(11)$ & C9-C10-C1 & $122.49(10)$ \\
C9-C10-C5 & $118.71(11)$ & C1-C10-C5 & $118.80(11)$ \\
C12-C11-C16 & $118.61(11)$ & C12-C11-N1 & $121.56(10)$ \\
C16-C11-N1 & $119.83(11)$ & C13-C12-C11 & $120.34(11)$ \\
C14-C13-C12 & $120.87(12)$ & C13-C14-C15 & $118.96(11)$ \\
C14-C15-C16 & $120.92(11)$ & C15-C16-C11 & $120.27(11)$ \\
C22-C17-C18 & $119.65(12)$ & C22-C17-N1 & $118.91(11)$ \\
C18-C17-N1 & $121.42(11)$ & C19-C18-C17 & $120.21(12)$ \\
C20-C19-C18 & $120.03(12)$ & C19-C20-C21 & $119.85(12)$ \\
C20-C21-C22 & $120.37(12)$ & C17-C22-C21 & $119.87(12)$ \\
C24-C23-C28 & $119.18(12)$ & C24-C23-N2 & $120.81(11)$ \\
C28-C23-N2 & $119.99(11)$ & C25-C24-C23 & $120.12(13)$ \\
C26-C25-C24 & $120.26(14)$ & C27-C26-C25 & $119.48(13)$ \\
C26-C27-C28 & $120.93(13)$ & C27-C28-C23 & $119.96(13)$ \\
C30-C29-C34 & $118.34(11)$ & C30-C29-N2 & $122.39(11)$ \\
C34-C29-N2 & $119.26(11)$ & C31-C30-C29 & $120.51(12)$ \\
C32-C31-C30 & $120.92(12)$ & C33-C32-C31 & $118.89(12)$ \\
C32-C33-C34 & $120.71(12)$ & C33-C34-C29 & $120.61(12)$ \\
C11-N1-C1 & $120.76(10)$ & C11-N1-C17 & $119.53(9)$ \\
C29-N2-C4 & $118.33(9)$ & C23-N2-C4 & $116.36(9)$ \\
\hline
\end{tabular}

Table S3-5. Anisotropic Atomic Displacement Parameters (Å2) for 1.

The anisotropic atomic displacement factor exponent takes the form: $-2 \pi 2\left[h 2 a^{*} 2 U 11+\ldots+2 h k a^{*} b^{*}\right.$ U12 ]

$$
\begin{array}{llllll}
U_{11} & U_{22} & U_{33} & U_{23} & U_{13} & U_{12}
\end{array}
$$




\begin{tabular}{|c|c|c|c|c|c|c|}
\hline C1 & $0.0150(6)$ & $0.0216(7)$ & $0.0151(6)$ & $0.0005(5)$ & $0.0047(5)$ & $-0.0032(5)$ \\
\hline $\mathrm{C} 2$ & $0.0226(6)$ & $0.0234(7)$ & $0.0190(6)$ & $-0.0034(5)$ & $0.0054(5)$ & $0.0052(5)$ \\
\hline C3 & $0.0226(6)$ & $0.0229(7)$ & $0.0190(6)$ & $0.0026(5)$ & $0.0019(5)$ & $0.0053(5)$ \\
\hline $\mathrm{C} 4$ & $0.0150(6)$ & $0.0211(7)$ & $0.0145(6)$ & $0.0008(5)$ & $0.0036(5)$ & $-0.0007(5)$ \\
\hline C5 & $0.0118(6)$ & $0.0187(6)$ & $0.0169(6)$ & $0.0001(5)$ & $0.0043(4)$ & $-0.0036(5)$ \\
\hline C6 & $0.0181(6)$ & $0.0205(7)$ & $0.0173(6)$ & $-0.0014(5)$ & $0.0048(5)$ & $-0.0038(5)$ \\
\hline $\mathrm{C7}$ & $0.0261(7)$ & $0.0182(7)$ & $0.0252(7)$ & $-0.0029(5)$ & $0.0068(5)$ & $0.0007(5)$ \\
\hline C8 & $0.0267(7)$ & $0.0188(7)$ & $0.0256(7)$ & $0.0045(5)$ & $0.0032(5)$ & $0.0037(6)$ \\
\hline C9 & $0.0207(6)$ & $0.0211(7)$ & $0.0177(6)$ & $0.0027(5)$ & $0.0037(5)$ & $-0.0015(5)$ \\
\hline C10 & $0.0131(6)$ & $0.0190(6)$ & $0.0159(6)$ & $0.0002(5)$ & $0.0038(4)$ & $-0.0044(5)$ \\
\hline C11 & $0.0167(6)$ & $0.0166(6)$ & $0.0179(6)$ & $0.0005(5)$ & $0.0054(5)$ & $0.0021(5)$ \\
\hline C12 & $0.0194(6)$ & $0.0207(7)$ & $0.0157(6)$ & $-0.0029(5)$ & $0.0041(5)$ & $0.0010(5)$ \\
\hline C13 & $0.0186(6)$ & $0.0193(7)$ & $0.0252(7)$ & $-0.0041(5)$ & $0.0068(5)$ & $-0.0008(5)$ \\
\hline C14 & $0.0234(6)$ & $0.0170(6)$ & $0.0249(7)$ & $0.0021(5)$ & $0.0121(5)$ & $0.0017(5)$ \\
\hline C15 & $0.0244(6)$ & $0.0208(7)$ & $0.0147(6)$ & $0.0018(5)$ & $0.0053(5)$ & $0.0049(5)$ \\
\hline C16 & $0.0188(6)$ & $0.0198(7)$ & $0.0182(6)$ & $-0.0008(5)$ & $0.0024(5)$ & $-0.0001(5)$ \\
\hline C17 & $0.0200(6)$ & $0.0203(6)$ & $0.0113(5)$ & $0.0029(5)$ & $0.0027(5)$ & $-0.0032(5)$ \\
\hline C18 & $0.0191(6)$ & $0.0251(7)$ & $0.0198(6)$ & $0.0007(5)$ & $0.0060(5)$ & $-0.0020(5)$ \\
\hline C19 & $0.0296(7)$ & $0.0214(7)$ & $0.0197(6)$ & $-0.0010(5)$ & $0.0061(5)$ & $-0.0012(6)$ \\
\hline C20 & $0.0296(7)$ & $0.0229(7)$ & $0.0189(6)$ & $0.0025(5)$ & $-0.0002(5)$ & $-0.0084(6)$ \\
\hline $\mathrm{C} 21$ & $0.0183(6)$ & $0.0311(8)$ & $0.0238(7)$ & $0.0049(6)$ & $0.0012(5)$ & $-0.0054(6)$ \\
\hline $\mathrm{C} 22$ & $0.0192(6)$ & $0.0252(7)$ & $0.0196(6)$ & $0.0026(5)$ & $0.0041(5)$ & $0.0006(5)$ \\
\hline C23 & $0.0197(6)$ & $0.0193(7)$ & $0.0187(6)$ & $0.0062(5)$ & $0.0053(5)$ & $0.0045(5)$ \\
\hline $\mathrm{C} 24$ & $0.0232(7)$ & $0.0280(8)$ & $0.0243(7)$ & $0.0076(6)$ & $0.0033(5)$ & $0.0006(6)$ \\
\hline $\mathrm{C} 25$ & $0.0213(7)$ & $0.0396(9)$ & $0.0414(8)$ & $0.0160(7)$ & $0.0030(6)$ & $-0.0033(6)$ \\
\hline C26 & $0.0258(7)$ & $0.0446(9)$ & $0.0433(9)$ & $0.0225(7)$ & $0.0188(7)$ & $0.0092(7)$ \\
\hline $\mathrm{C} 27$ & $0.0348(8)$ & $0.0332(8)$ & $0.0280(7)$ & $0.0126(6)$ & $0.0168(6)$ & $0.0132(7)$ \\
\hline C28 & $0.0280(7)$ & $0.0231(7)$ & $0.0215(7)$ & $0.0050(5)$ & $0.0084(5)$ & $0.0073(6)$ \\
\hline C29 & $0.0196(6)$ & $0.0166(6)$ & $0.0147(6)$ & $-0.0041(5)$ & $0.0027(5)$ & $0.0009(5)$ \\
\hline C30 & $0.0228(6)$ & $0.0220(7)$ & $0.0185(6)$ & $0.0011(5)$ & $0.0044(5)$ & $-0.0010(5)$ \\
\hline C31 & $0.0291(7)$ & $0.0311(8)$ & $0.0180(6)$ & $0.0029(6)$ & $-0.0004(5)$ & $0.0034(6)$ \\
\hline C32 & $0.0212(7)$ & $0.0339(8)$ & $0.0243(7)$ & $-0.0050(6)$ & $-0.0031(5)$ & $0.0007(6)$ \\
\hline C33 & $0.0205(7)$ & $0.0244(7)$ & $0.0284(7)$ & $-0.0062(6)$ & $0.0063(5)$ & $-0.0057(5)$ \\
\hline C34 & $0.0243(6)$ & $0.0181(6)$ & $0.0179(6)$ & $-0.0017(5)$ & $0.0059(5)$ & $-0.0018(5)$ \\
\hline
\end{tabular}




$\begin{array}{lllllll}\text { N1 } & 0.0210(5) & 0.0208(6) & 0.0134(5) & -0.0012(4) & 0.0048(4) & -0.0044(4) \\ \text { N2 } & 0.0169(5) & 0.0246(6) & 0.0130(5) & 0.0015(4) & 0.0041(4) & 0.0014(4)\end{array}$

Table S3-6. Hydrogen Atomic Coordinates and Isotropic Atomic Displacement Parameters ( $\AA 2)$ for 1.

$\begin{array}{lcccc} & \mathbf{x} / \mathbf{a} & \mathbf{y} / \mathbf{b} & \mathbf{z} / \mathbf{c} & \mathbf{U}(\mathbf{e q}) \\ \mathrm{H} 2 & 0.6139 & 1.0904 & 0.9752 & 0.026 \\ \mathrm{H} 3 & 0.5853 & 1.092 & 0.8887 & 0.026 \\ \mathrm{H} 6 & 0.7538 & 0.5878 & 0.845 & 0.022 \\ \mathrm{H} 7 & 0.8454 & 0.3618 & 0.8878 & 0.027 \\ \mathrm{H} 8 & 0.8779 & 0.3629 & 0.9747 & 0.028 \\ \mathrm{H} 9 & 0.8159 & 0.5888 & 1.0181 & 0.024 \\ \mathrm{H} 12 & 0.5483 & 0.6684 & 1.0099 & 0.022 \\ \mathrm{H} 13 & 0.4403 & 0.5117 & 1.0598 & 0.025 \\ \mathrm{H} 14 & 0.4811 & 0.529 & 1.1458 & 0.025 \\ \mathrm{H} 15 & 0.6321 & 0.705 & 1.1816 & 0.024 \\ \mathrm{H} 16 & 0.7445 & 0.8573 & 1.1325 & 0.023 \\ \mathrm{H} 18 & 0.6352 & 1.1438 & 1.0764 & 0.025 \\ \mathrm{H} 19 & 0.7287 & 1.3928 & 1.1067 & 0.028 \\ \mathrm{H} 20 & 0.9285 & 1.4279 & 1.1043 & 0.029 \\ \mathrm{H} 21 & 1.0339 & 1.2157 & 1.0708 & 0.029 \\ \mathrm{H} 22 & 0.9412 & 0.9655 & 1.0409 & 0.025 \\ \mathrm{H} 24 & 0.842 & 1.0403 & 0.8649 & 0.03 \\ \mathrm{H} 25 & 1.0105 & 1.0992 & 0.8296 & 0.041 \\ \mathrm{H} 26 & 1.0241 & 1.0124 & 0.7489 & 0.044 \\ \mathrm{H} 27 & 0.8735 & 0.8546 & 0.7054 & 0.037 \\ \mathrm{H} 28 & 0.7078 & 0.7873 & 0.7408 & 0.029 \\ \mathrm{H} 30 & 0.5904 & 1.0476 & 0.7447 & 0.025 \\ \mathrm{H} 31 & 0.4034 & 1.1002 & 0.7042 & 0.032 \\ \mathrm{H} 32 & 0.2396 & 0.9963 & 0.7357 & 0.032 \\ \mathrm{H} 33 & 0.2656 & 0.8292 & 0.807 & 0.029 \\ \mathrm{H} & 0.4521 & 0.7716 & 0.8472 & 0.024\end{array}$




\section{Reference}

1 Noto, N.; Tanaka, Y.; Koike, T.; Akita, M. Strongly Reducing (Diarylamino)anthracene Catalyst for Metal-Free Visible-Light Photocatalytic Fluoroalkylation. ACS Catal. 2018, 8, 9408-9419.

2 (a) Prakash, G. K. S.; Ledneczki, I.; Chacko, S.; Olah, G. A. Direct Electrophilic Monofluoromethylation. Org. Lett. 2008, 10, 557-560. (b) Rydzik, A. M.; Leung, I. K. H.; Thalhammer, A.; Kochan, G. T.; Claridge, T. D. W.; Schofield, C. J. Fluoromethylated Derivatives of Carnitine Biosynthesis Intermediates - Synthesis and Applications. Chem. Commun. 2014, 50, 1175-1177. (c) Lohier, J.-F.; Glachet, T.; Marzag, H.; Gaumont, A.-C.; Reboul, V. Mechanistic Investigation of the NHSulfoximination of Sulfide. Evidence for $\lambda^{6}$-Sulfanenitrile Intermediates. Chem. Commun. 2017, 53, 2064-2067. (d) Shen, X.; Zhang, W.; Zhang, L.; Luo, T.; Wan, X.; Gu, Y.; Hu, J. Enantioselective Synthesis of Cyclopropanes That Contain Fluorinated Tertiary Stereogenic Carbon Centers: A Chiral a-Fluoro Carbanion Strategy. Angew. Chem. Int. Ed. 2012, 51, 6966-6970.

3 a) Shen, R.; Yang, J.; Zhu, S.; Chen, C.; Wu, L. Gold(I)-Catalyzed Decarboxylation of Propargyl Carbonates: Reactivity Reversal of the Gold Catalyst from $\pi$-Lewis Acidity to $\sigma$-Lewis Acidity. Adv. Synth. Catal. 2015, 357, 1259-1269. (b) Lei, C.; Yip, Y.; Zhou, J. J. S. Nickel-Catalyzed Direct Synthesis of Aryl Olefins from Ketones and Organoboron Reagents under Neutral Conditions. J. Am. Chem. Soc. 2017, 139, 6086-6089. 


\section{NMR Spectra}

${ }^{1} \mathrm{H}$ NMR $\left(400 \mathrm{MHz}, \mathrm{CD}_{2} \mathrm{Cl}_{2}\right.$, rt $)$

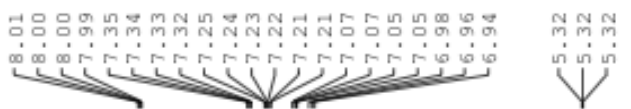

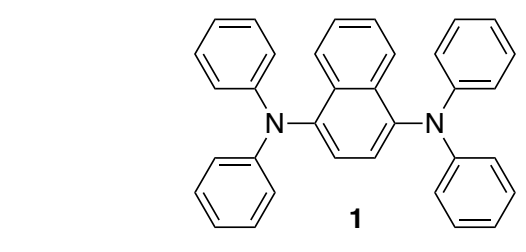

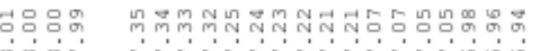

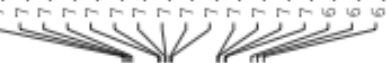
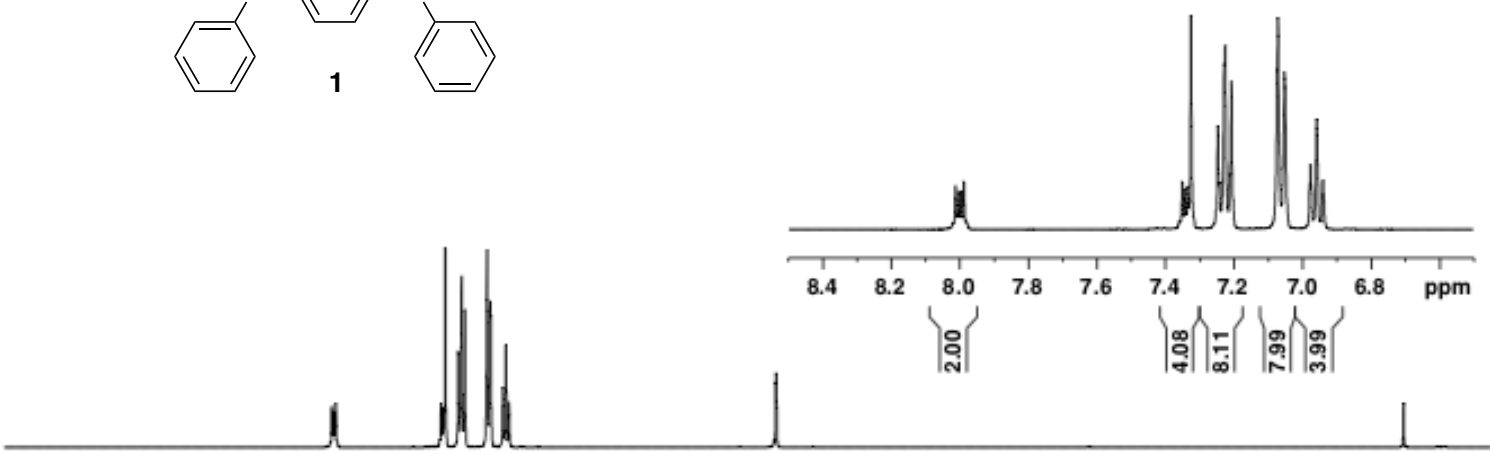

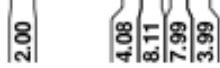

${ }^{13} \mathrm{C}$ NMR $\left(125 \mathrm{MHz}, \mathrm{CD}_{2} \mathrm{Cl}_{2}, \mathrm{rt}\right)$
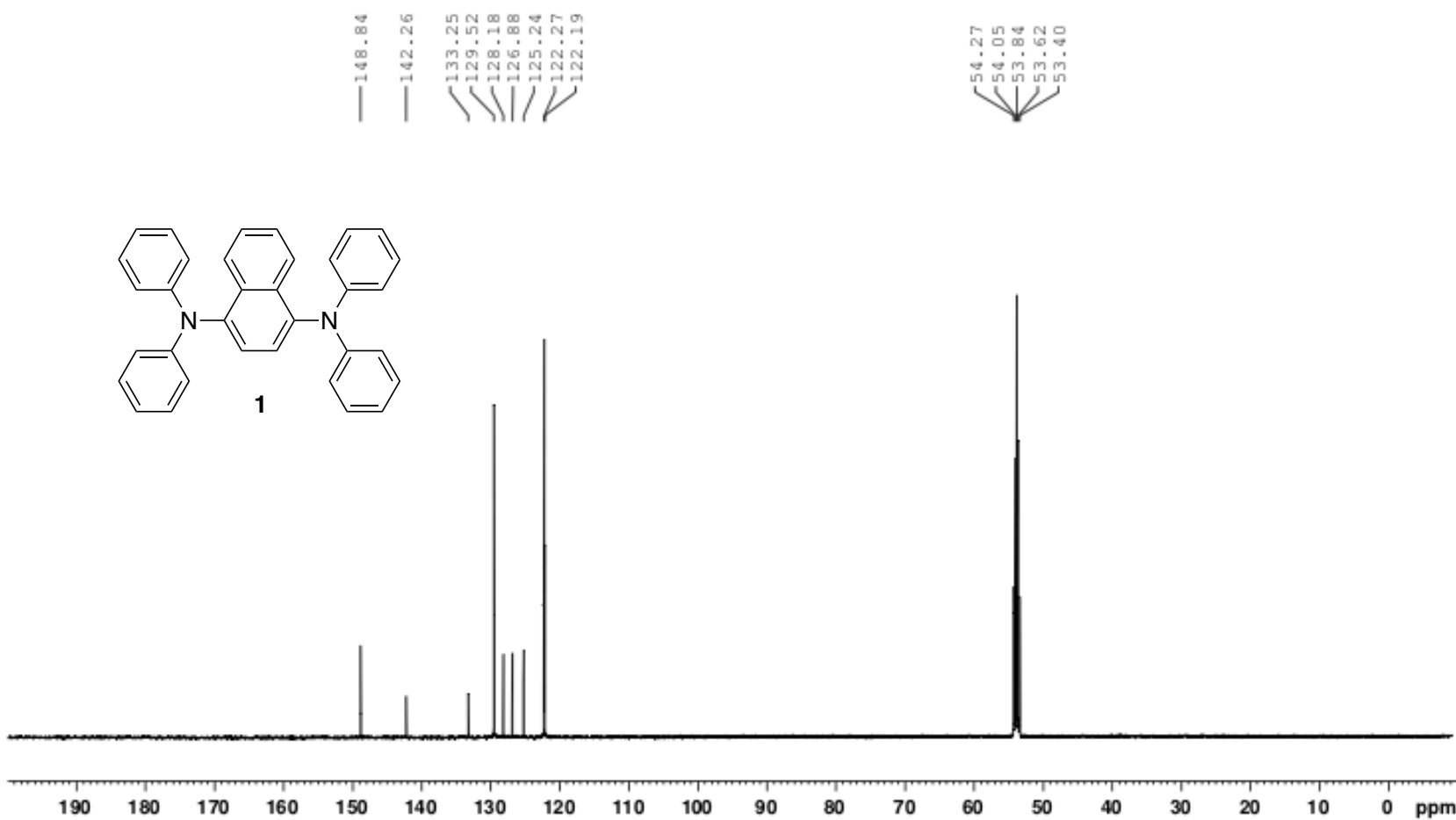
${ }^{1} \mathrm{H}$ NMR $\left(400 \mathrm{MHz}, \mathrm{CDCl}_{3}, \mathrm{rt}\right)$

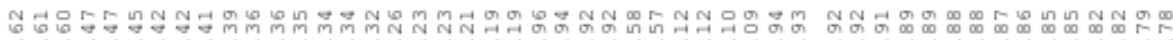

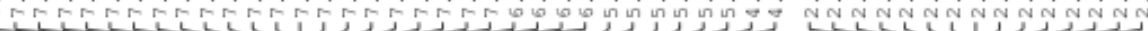<smiles>C=C1CC(c2ccccc2)Oc2ccccc21</smiles>
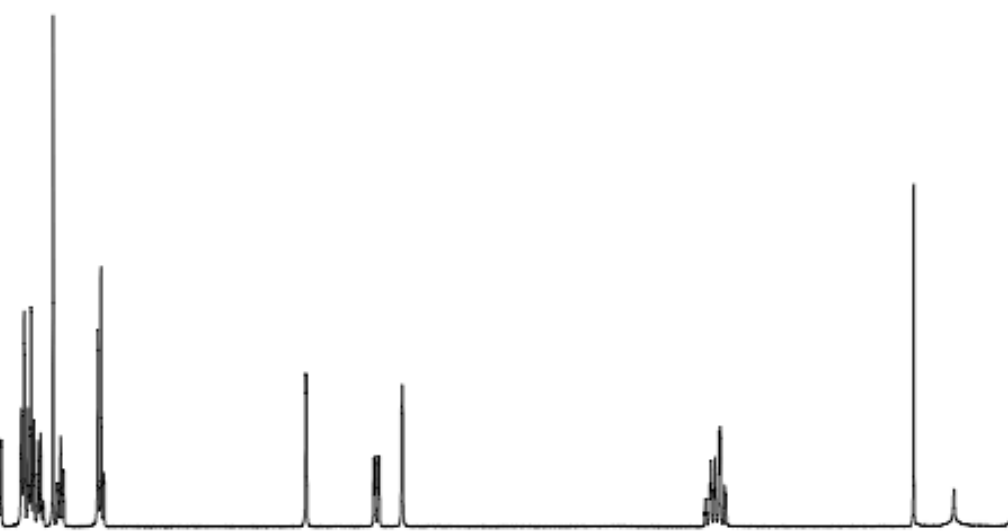

$\begin{array}{llll}9.5 & 9.0 & 8.5 & 8.0\end{array}$

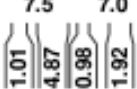

$\begin{array}{lllll}5.5 & 5.0 & 4.5 & 4.0 & 3.5\end{array}$

$\begin{array}{lll}0 & 2.5 & 2.0\end{array}$

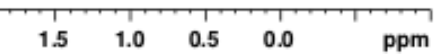

क्षे|

${ }^{13} \mathrm{C}$ NMR $\left(125 \mathrm{MHz}, \mathrm{CDCl}_{3}, \mathrm{rt}\right)$

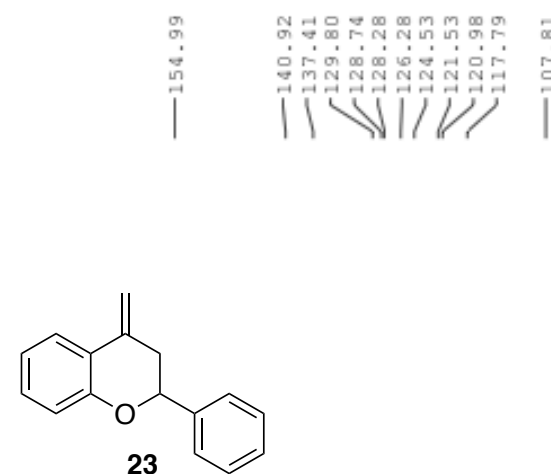

23

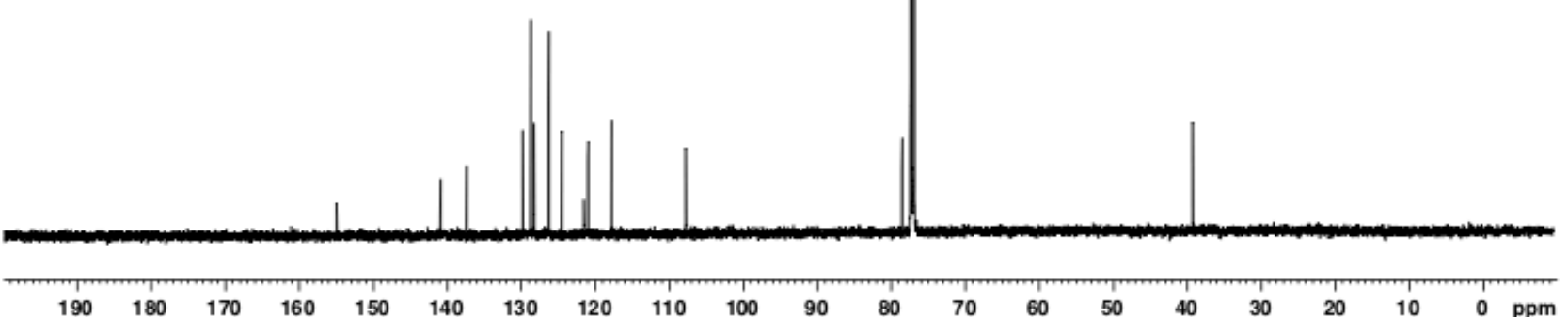


${ }^{1} \mathrm{H}$ NMR $\left(400 \mathrm{MHz}, \mathrm{CDCl}_{3}, \mathrm{rt}\right)$

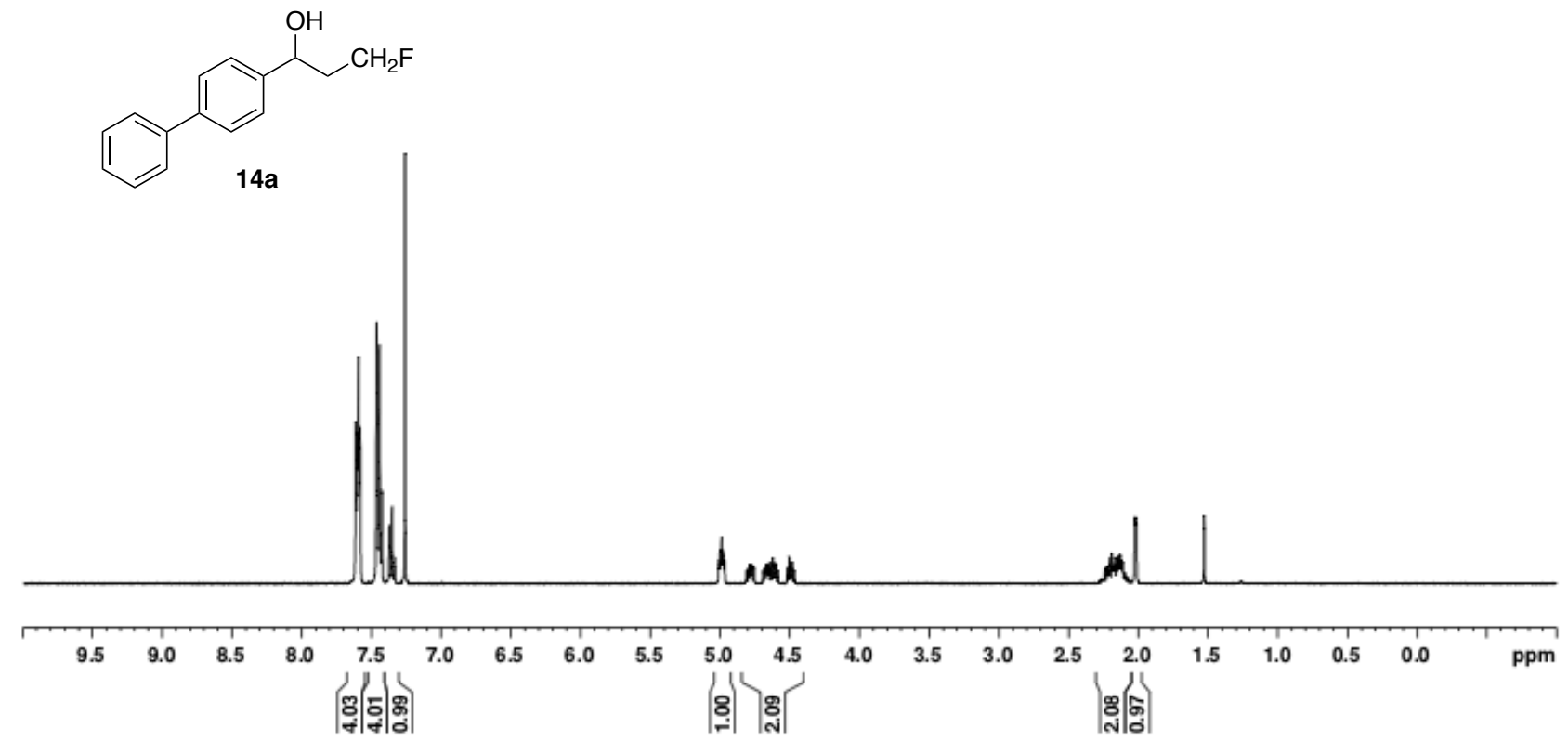

${ }^{13} \mathrm{C}$ NMR $\left(125 \mathrm{MHz}, \mathrm{CDCl}_{3}, \mathrm{rt}\right)$
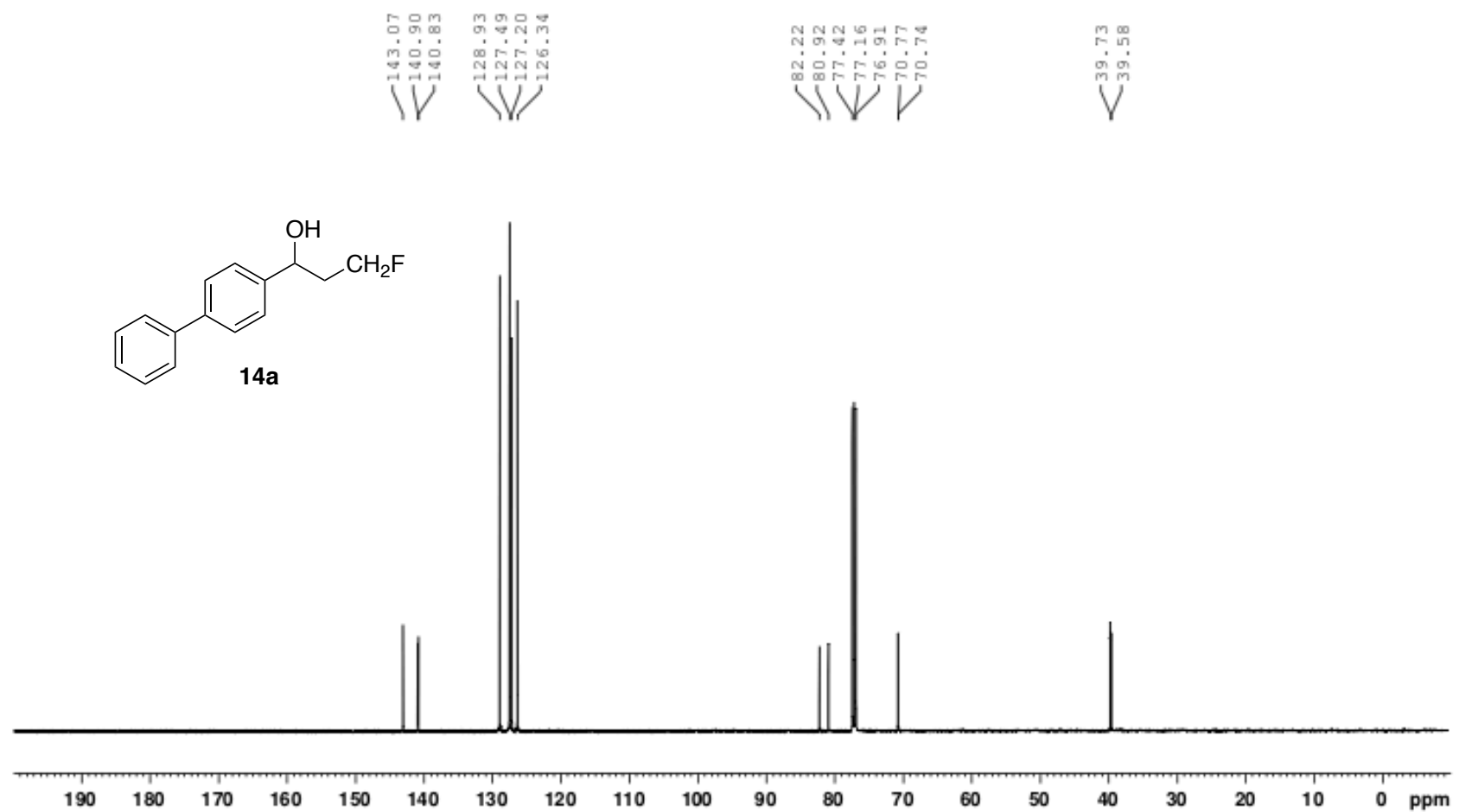
${ }^{19} \mathrm{~F} \mathrm{NMR}\left(376 \mathrm{MHz}, \mathrm{CDCl}_{3}, \mathrm{rt}\right)$

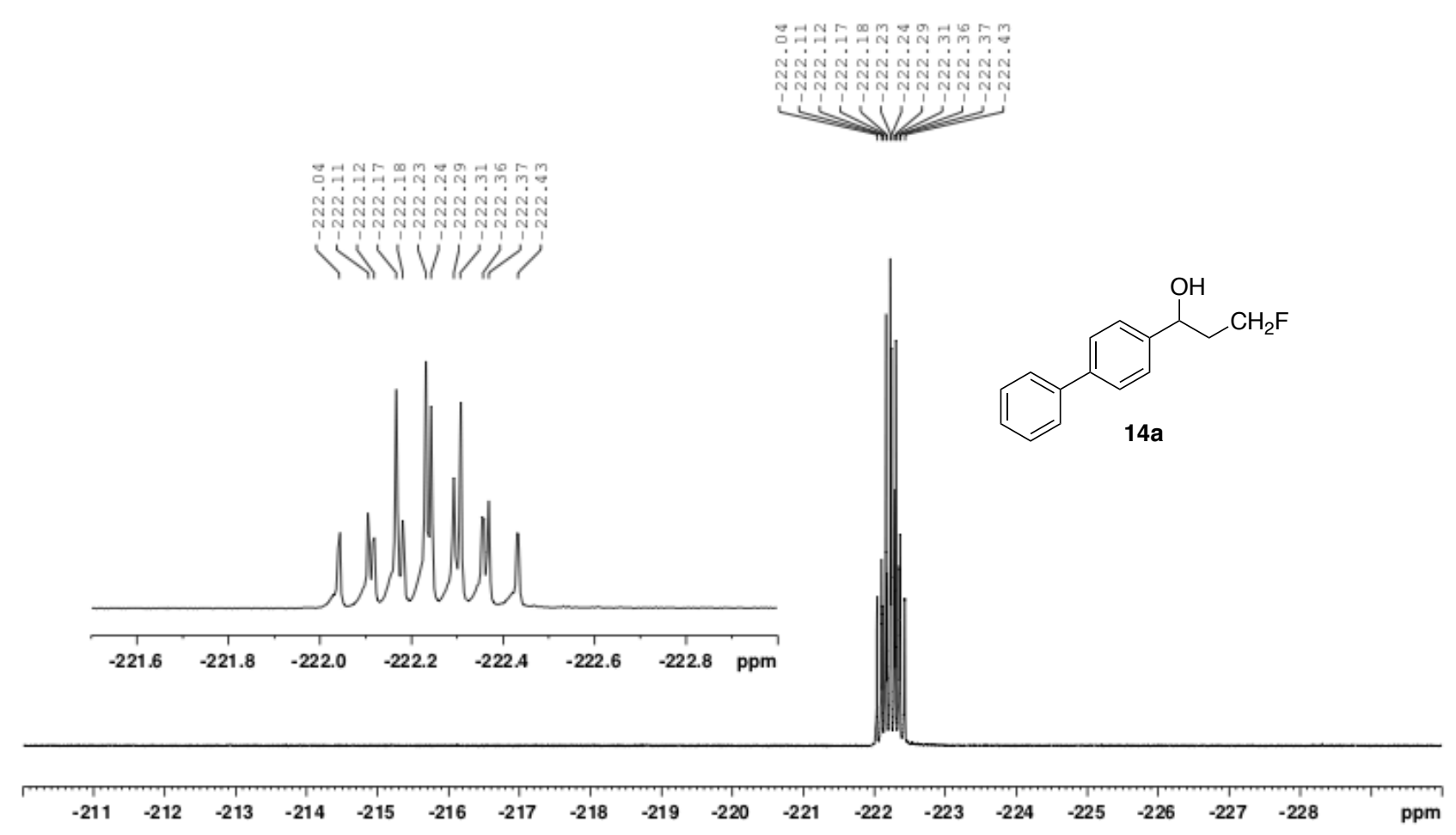

${ }^{1} \mathrm{H}$ NMR $\left(400 \mathrm{MHz}, \mathrm{CDCl}_{3}, \mathrm{rt}\right)$

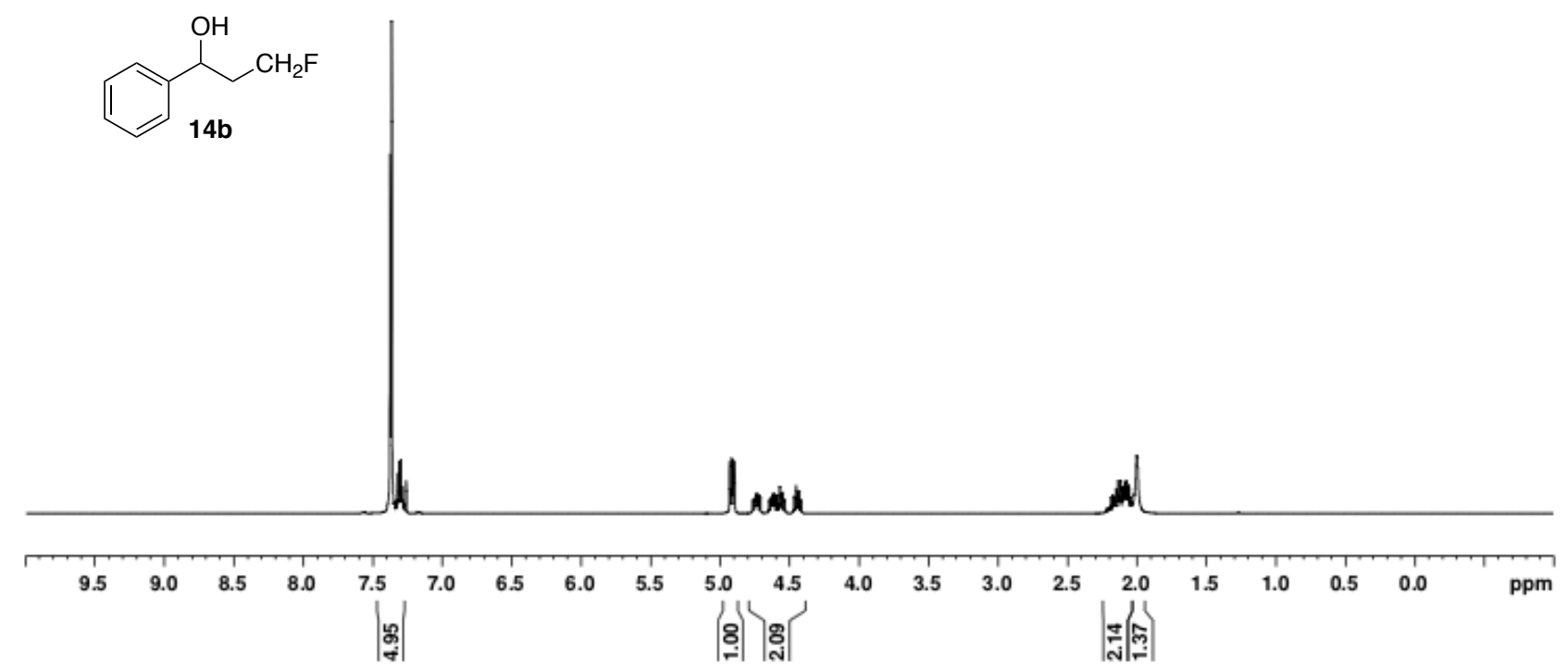


${ }^{13} \mathrm{C}$ NMR $\left(125 \mathrm{MHz}, \mathrm{CDCl}_{3}, \mathrm{rt}\right)$

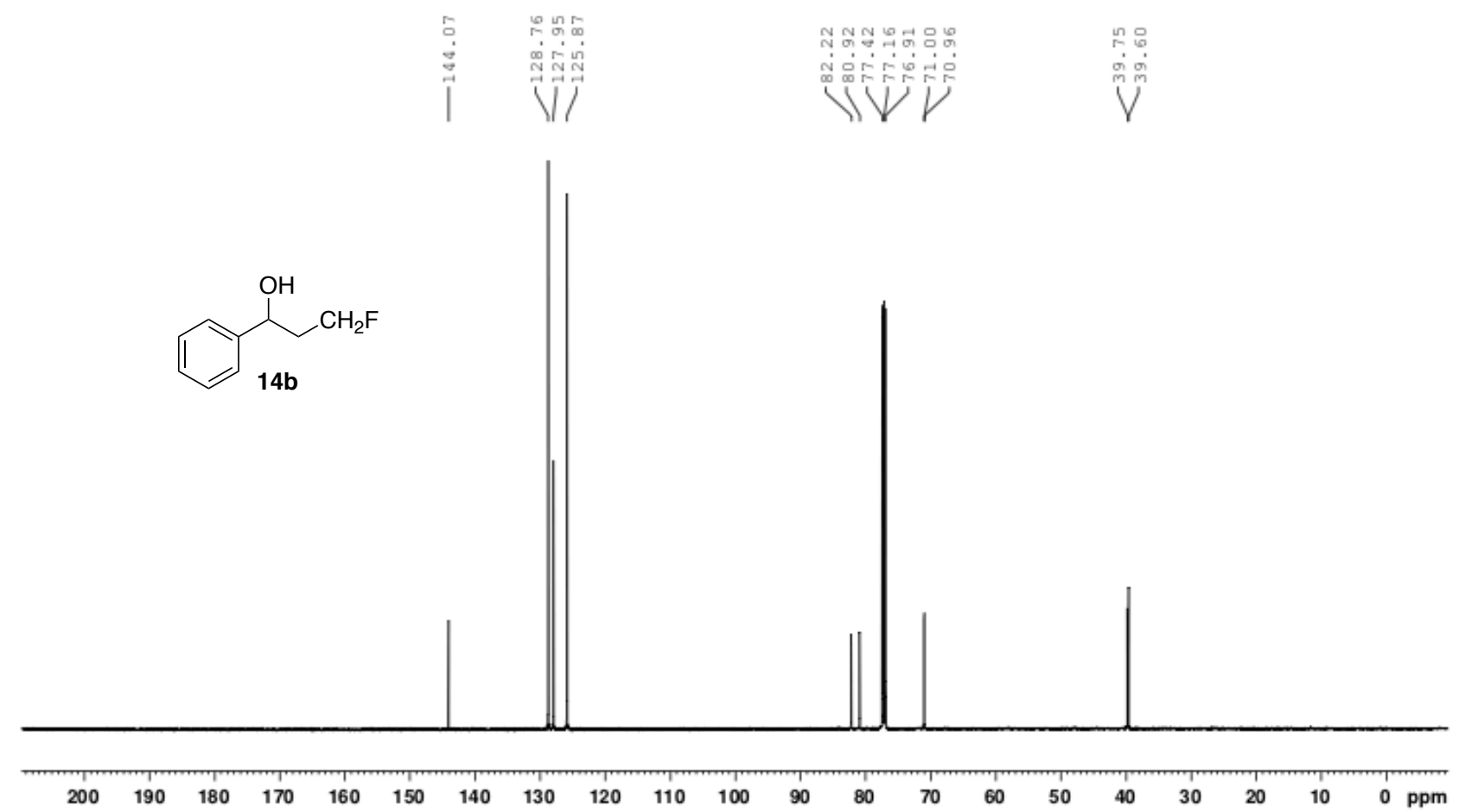

${ }^{19} \mathrm{~F} \mathrm{NMR}\left(376 \mathrm{MHz}, \mathrm{CDCl}_{3}, \mathrm{rt}\right)$

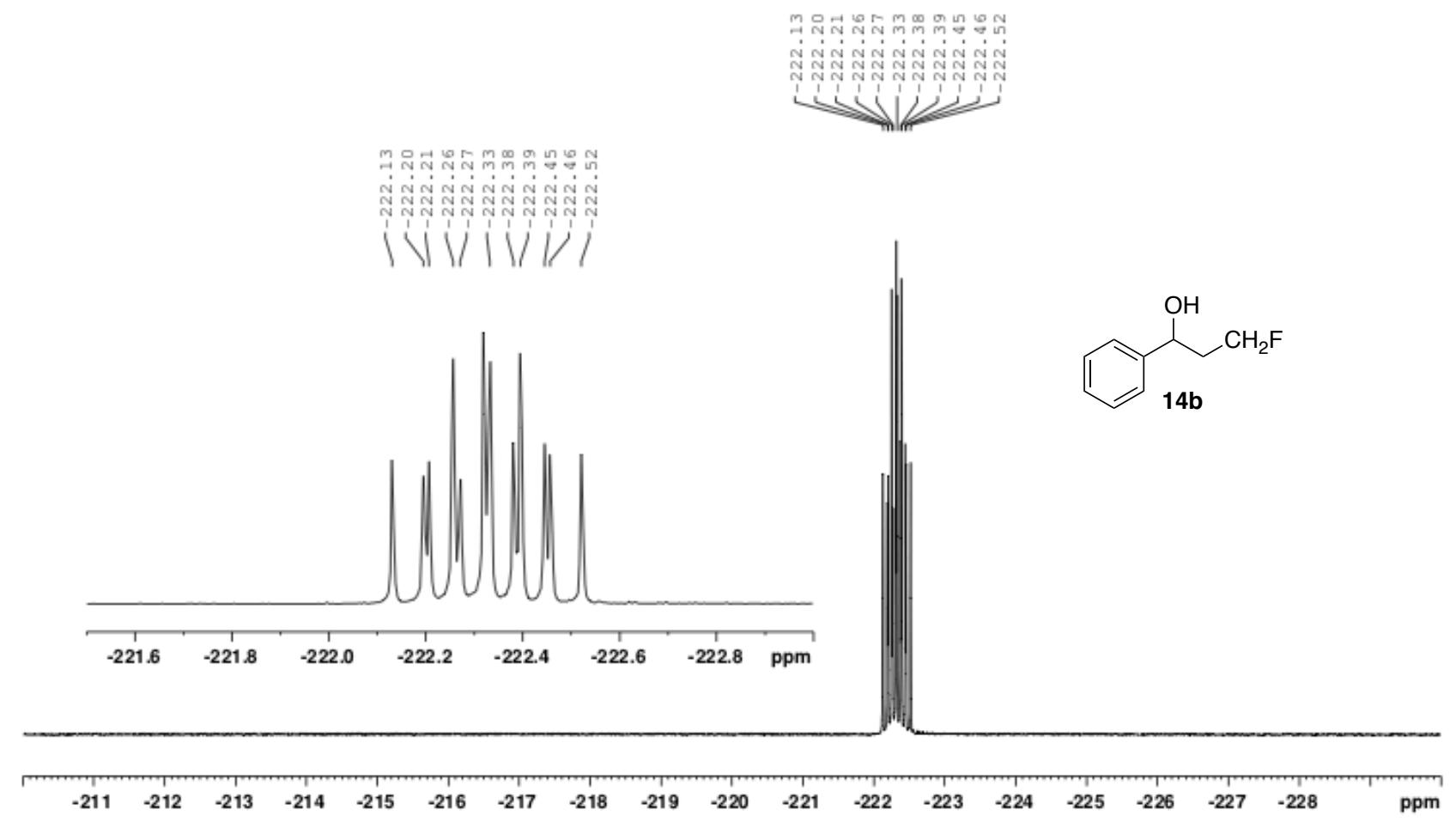


${ }^{1} \mathrm{H}$ NMR $\left(400 \mathrm{MHz}, \mathrm{CDCl}_{3}, \mathrm{rt}\right)$

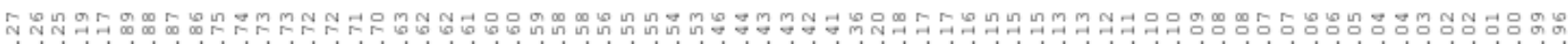

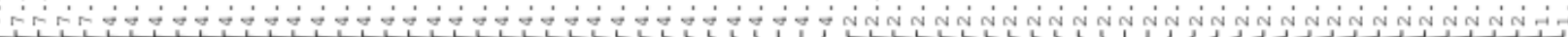<smiles>Cc1ccc(C(O)CCF)cc1</smiles>

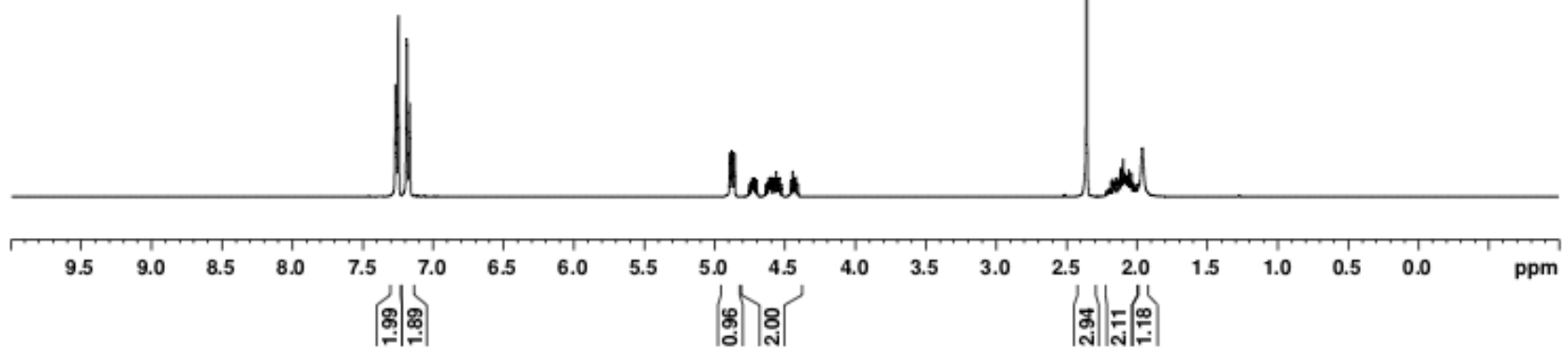

${ }^{13} \mathrm{C}$ NMR $\left(125 \mathrm{MHz}, \mathrm{CDCl}_{3}, \mathrm{rt}\right)$

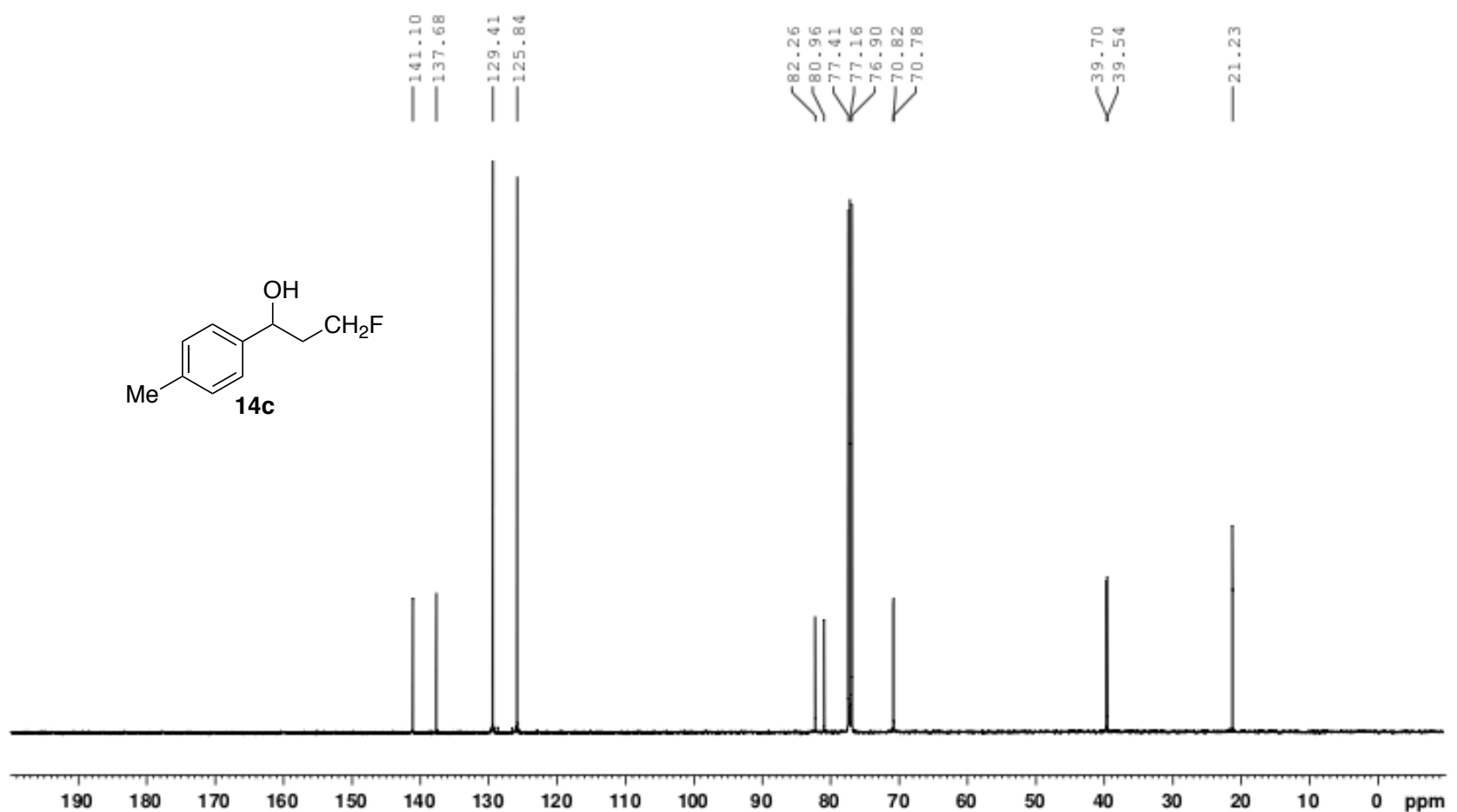


${ }^{19} \mathrm{~F} \mathrm{NMR}\left(376 \mathrm{MHz}, \mathrm{CDCl}_{3}, \mathrm{rt}\right)$

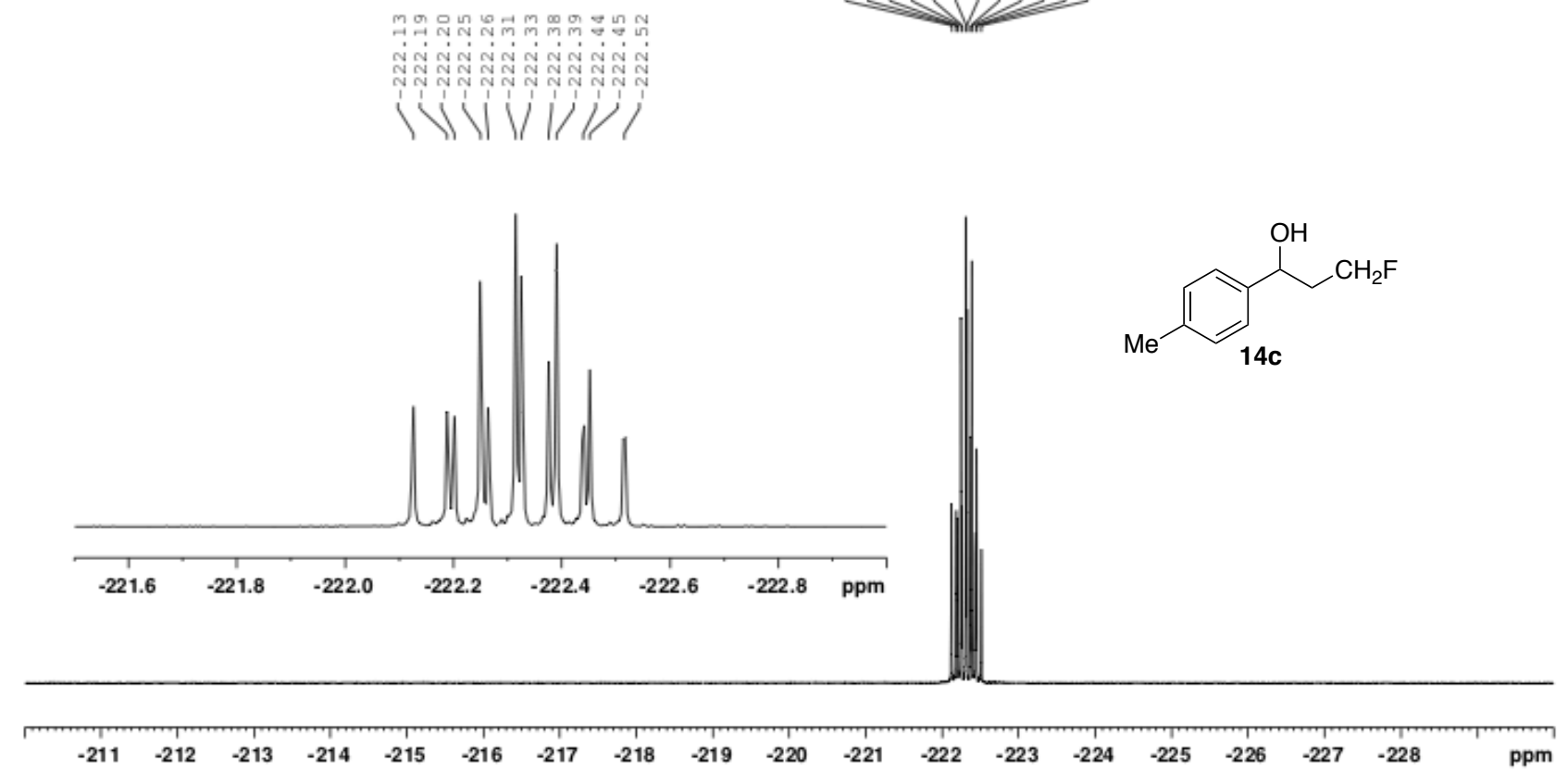

${ }^{1} \mathrm{H}$ NMR $\left(400 \mathrm{MHz}, \mathrm{CDCl}_{3}, \mathrm{rt}\right)$

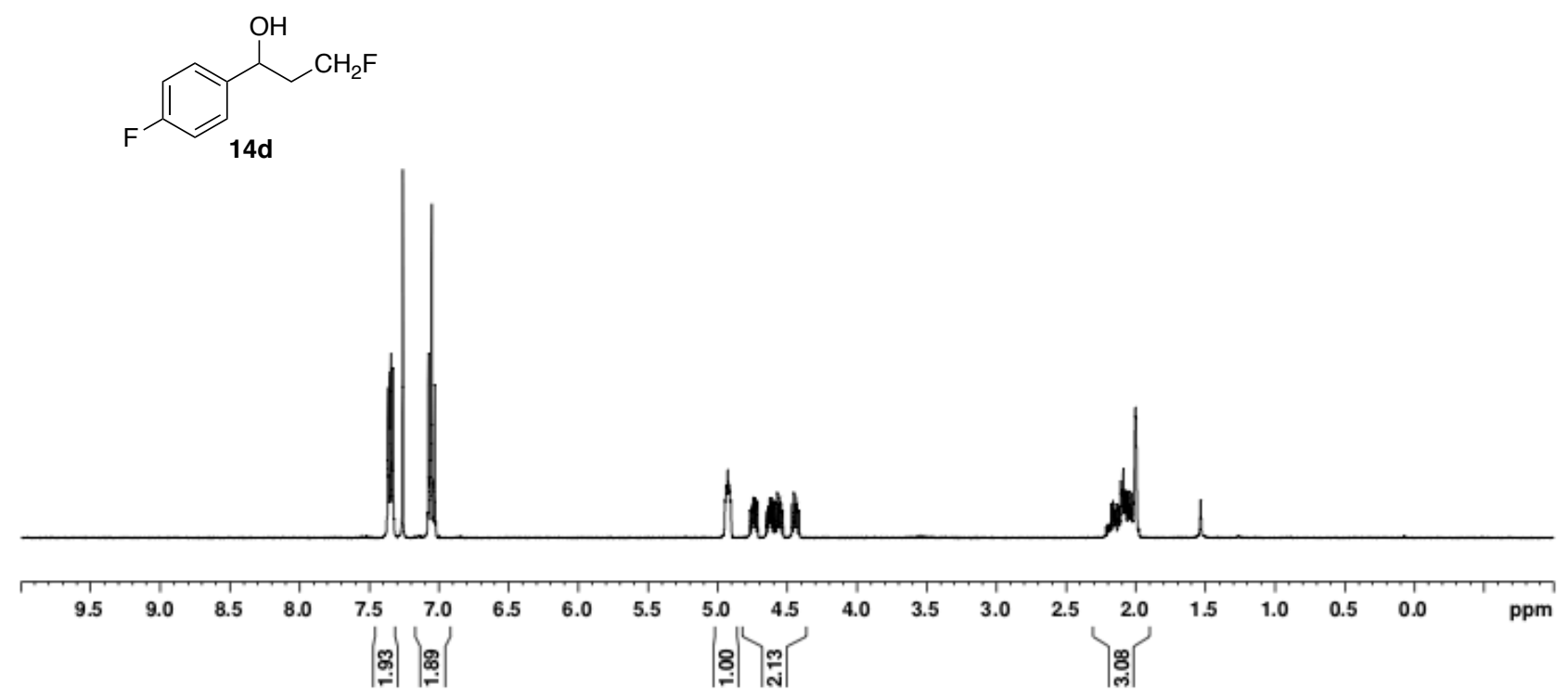


${ }^{13} \mathrm{C}$ NMR (125 MHz, $\left.\mathrm{CDCl}_{3}, \mathrm{rt}\right)$

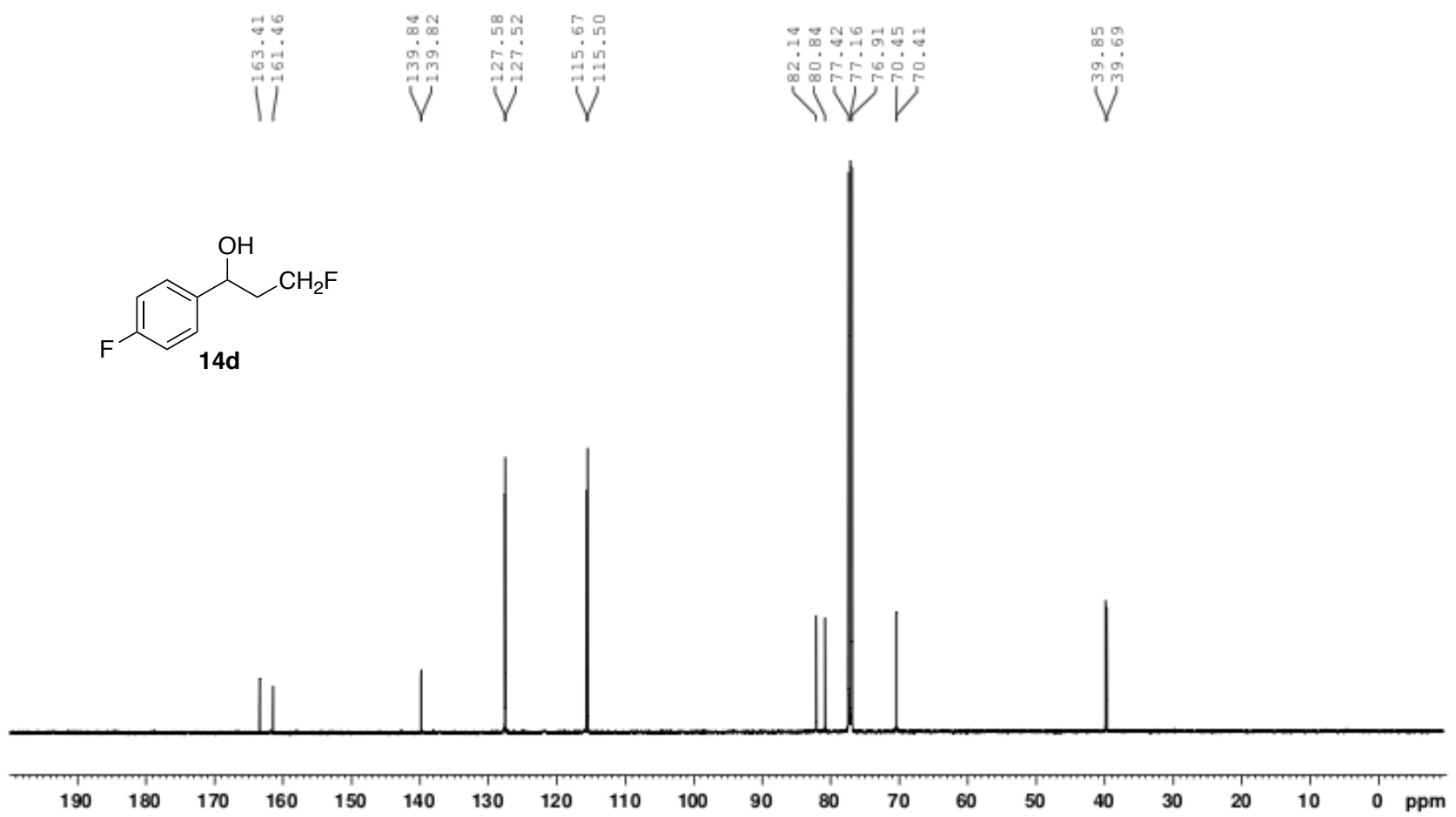

${ }^{19} \mathrm{~F} \mathrm{NMR}\left(376 \mathrm{MHz}, \mathrm{CDCl}_{3}, \mathrm{rt}\right)$

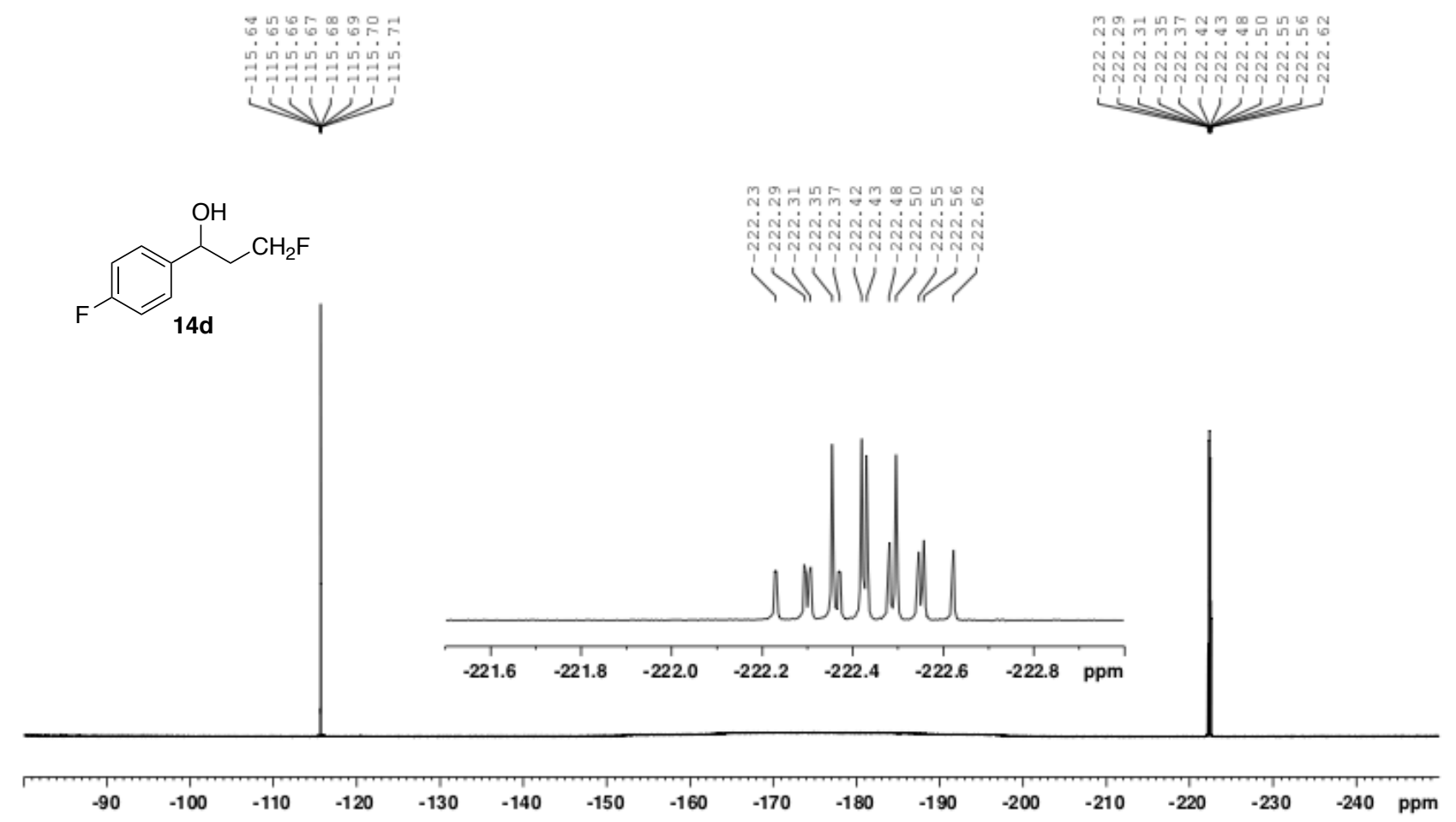


${ }^{1} \mathrm{H} \mathrm{NMR}\left(400 \mathrm{MHz}, \mathrm{CDCl}_{3}, \mathrm{rt}\right)$

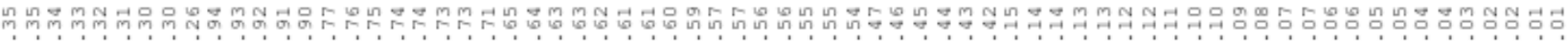
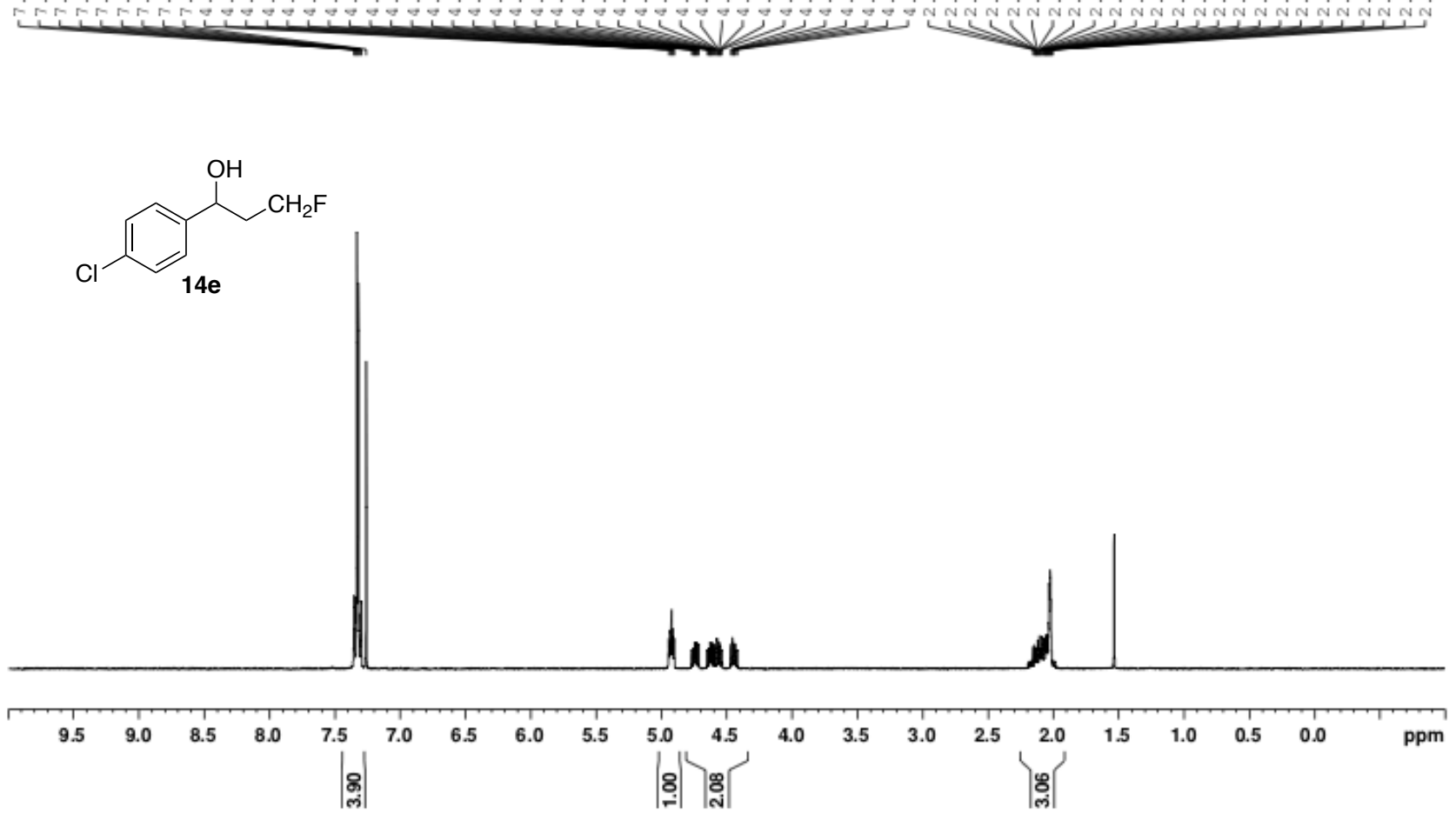

${ }^{13} \mathrm{C}$ NMR $\left(125 \mathrm{MHz}, \mathrm{CDCl}_{3}, \mathrm{rt}\right)$
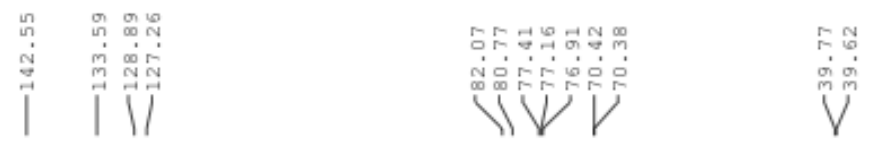<smiles>OC(CF)c1ccc(Cl)cc1</smiles>
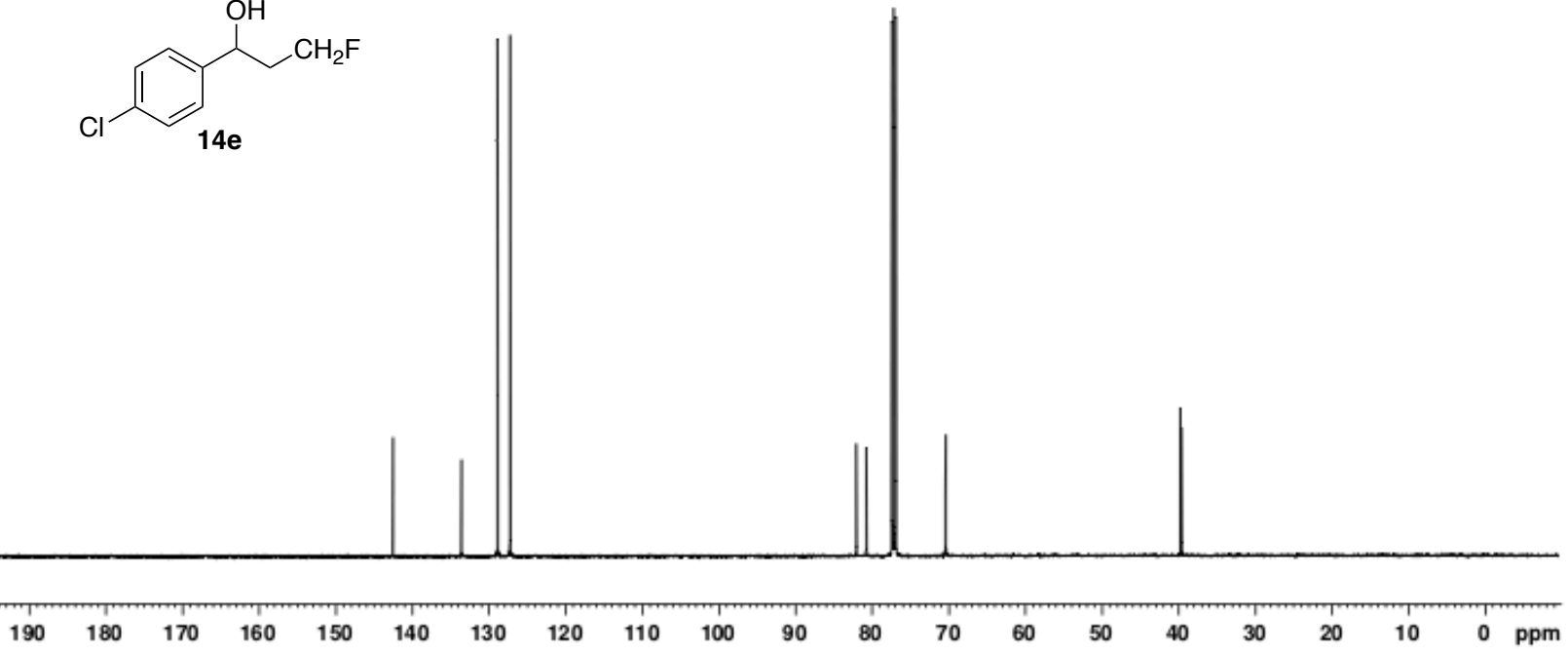
${ }^{19} \mathrm{~F} \mathrm{NMR}\left(376 \mathrm{MHz}, \mathrm{CDCl}_{3}, \mathrm{rt}\right)$

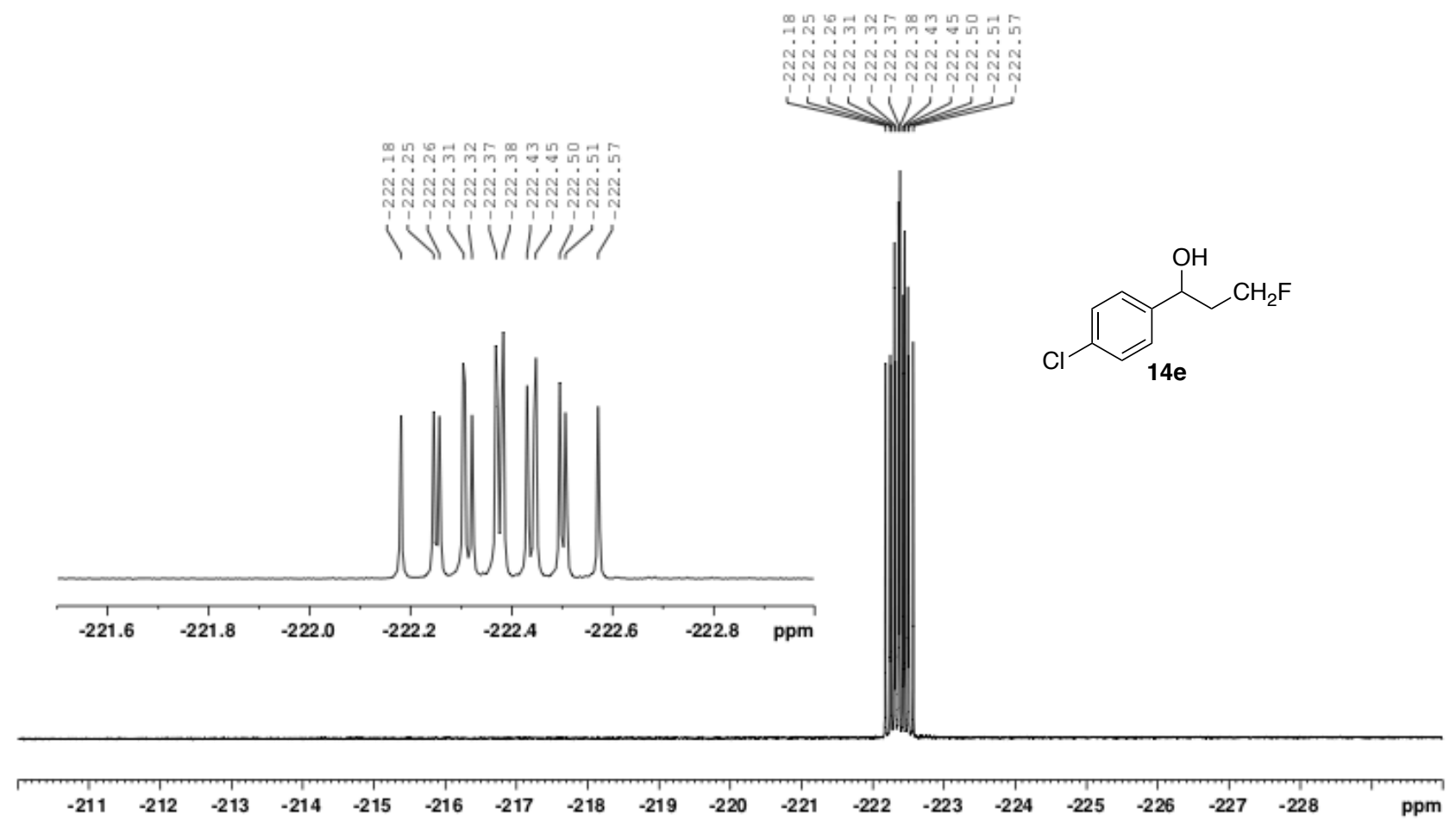

$\left.{ }^{1} \mathrm{H} \mathrm{NMR} \mathrm{(400} \mathrm{MHz,} \mathrm{CDCl}_{3}, \mathrm{rt}\right)$<smiles>OC(CCF)c1ccc(Br)cc1</smiles>

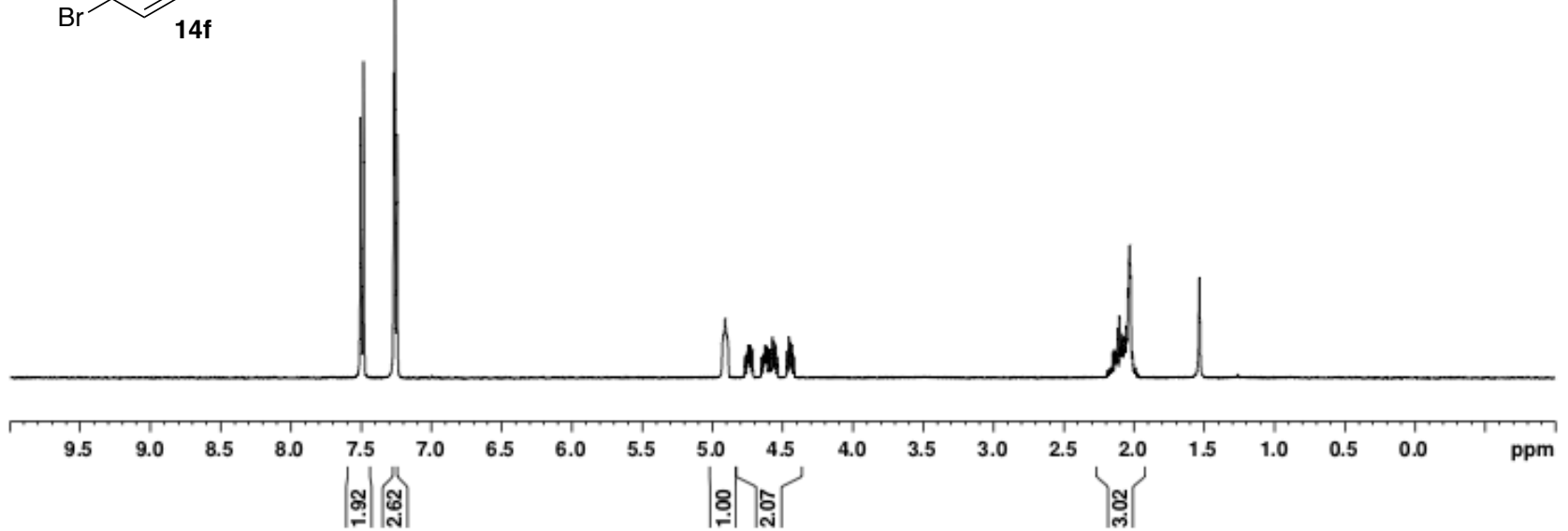


${ }^{13} \mathrm{C}$ NMR $\left(125 \mathrm{MHz}, \mathrm{CDCl}_{3}, \mathrm{rt}\right)$

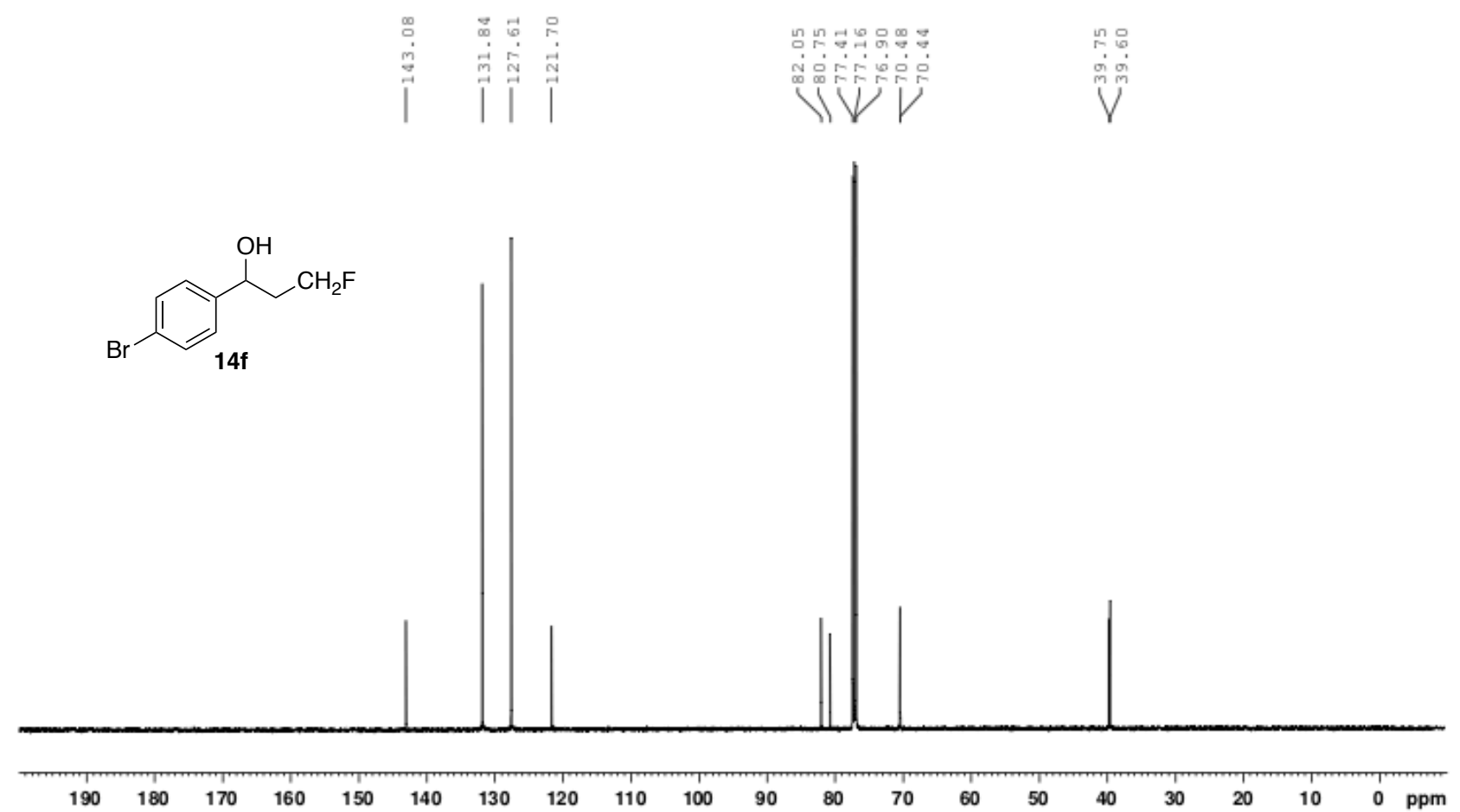

${ }^{19} \mathrm{~F} \mathrm{NMR}\left(376 \mathrm{MHz}, \mathrm{CDCl}_{3}, \mathrm{rt}\right)$

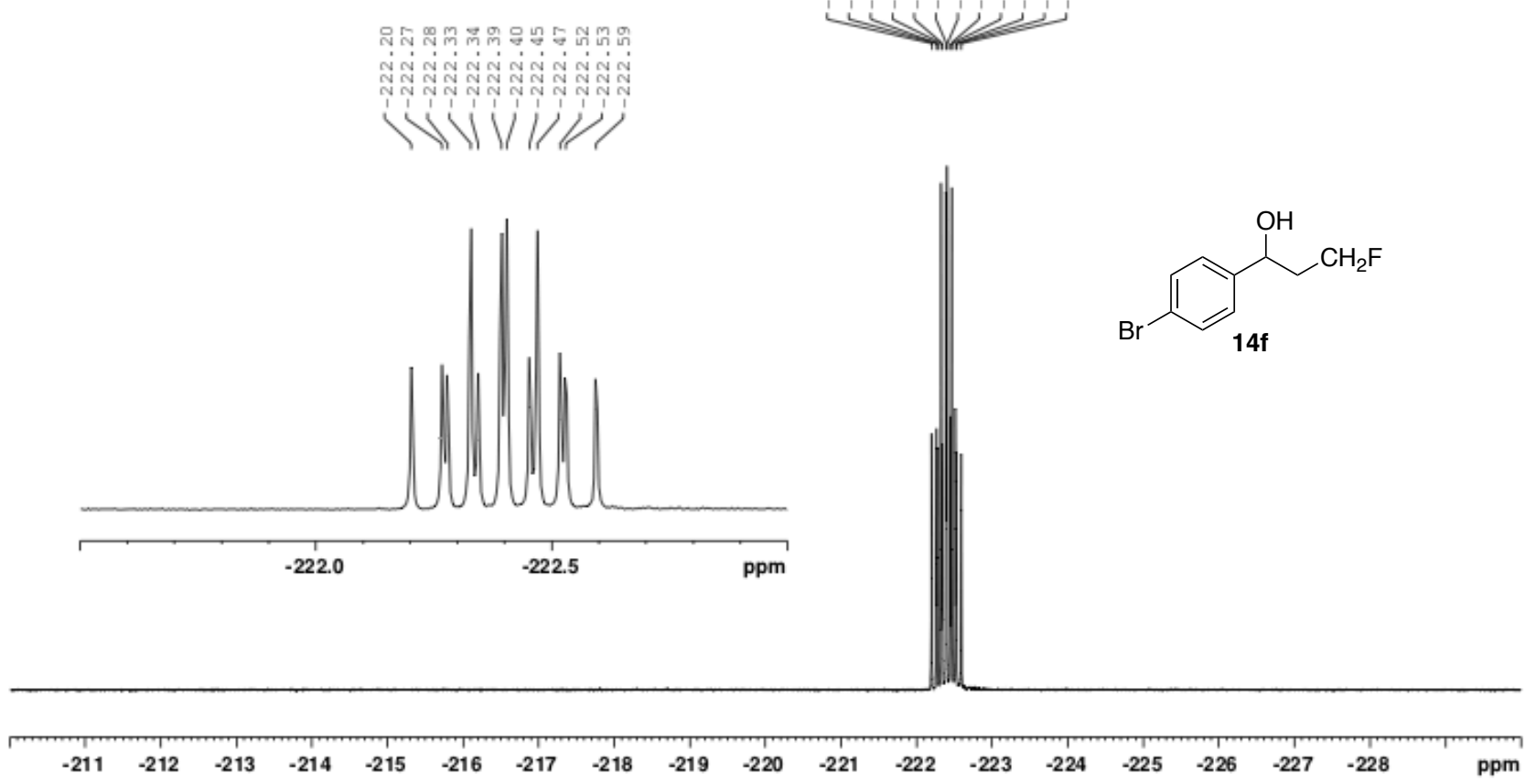


${ }^{1} \mathrm{H} \mathrm{NMR}\left(400 \mathrm{MHz}, \mathrm{CDCl}_{3}, \mathrm{rt}\right)$

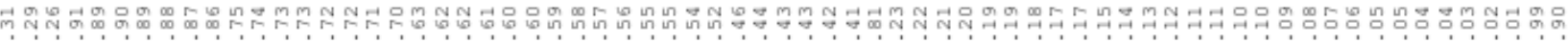

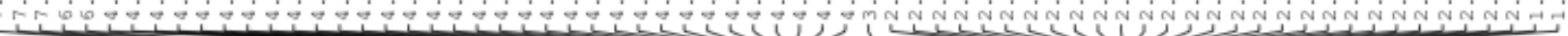

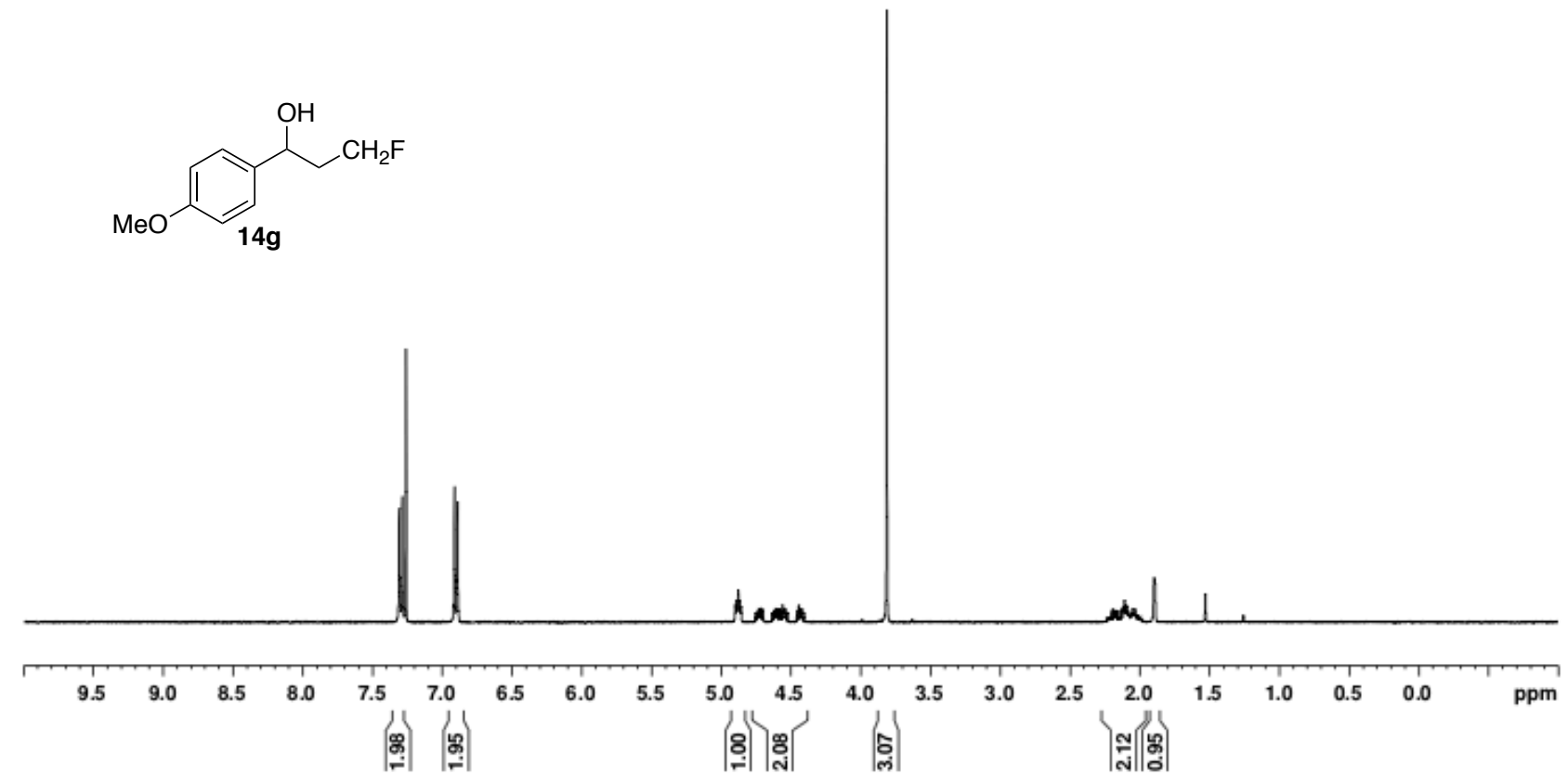

${ }^{13} \mathrm{C}$ NMR $\left(125 \mathrm{MHz}, \mathrm{CDCl}_{3}, \mathrm{rt}\right)$

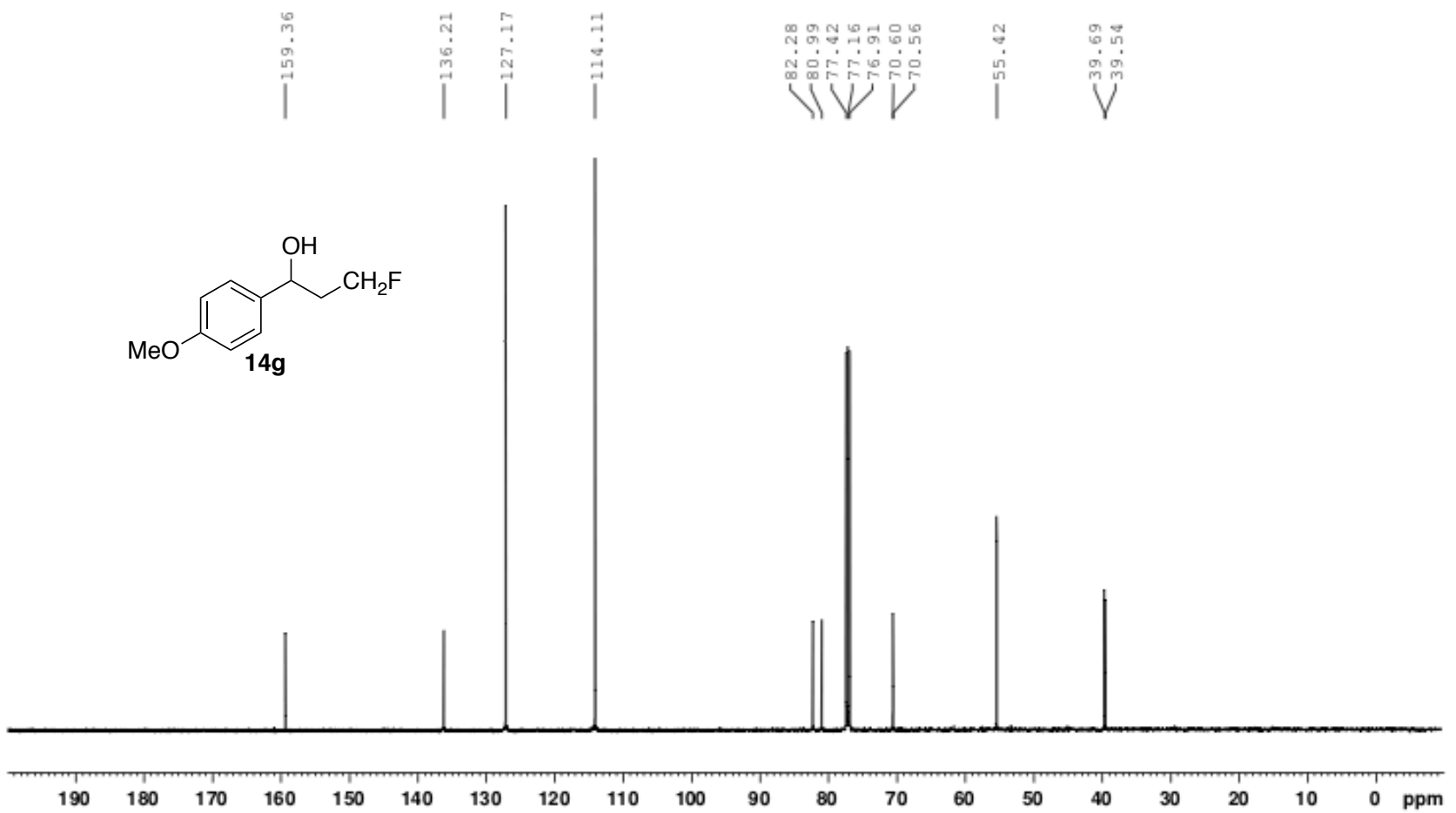


${ }^{19} \mathrm{~F} \mathrm{NMR}\left(376 \mathrm{MHz}, \mathrm{CDCl}_{3}, \mathrm{rt}\right)$

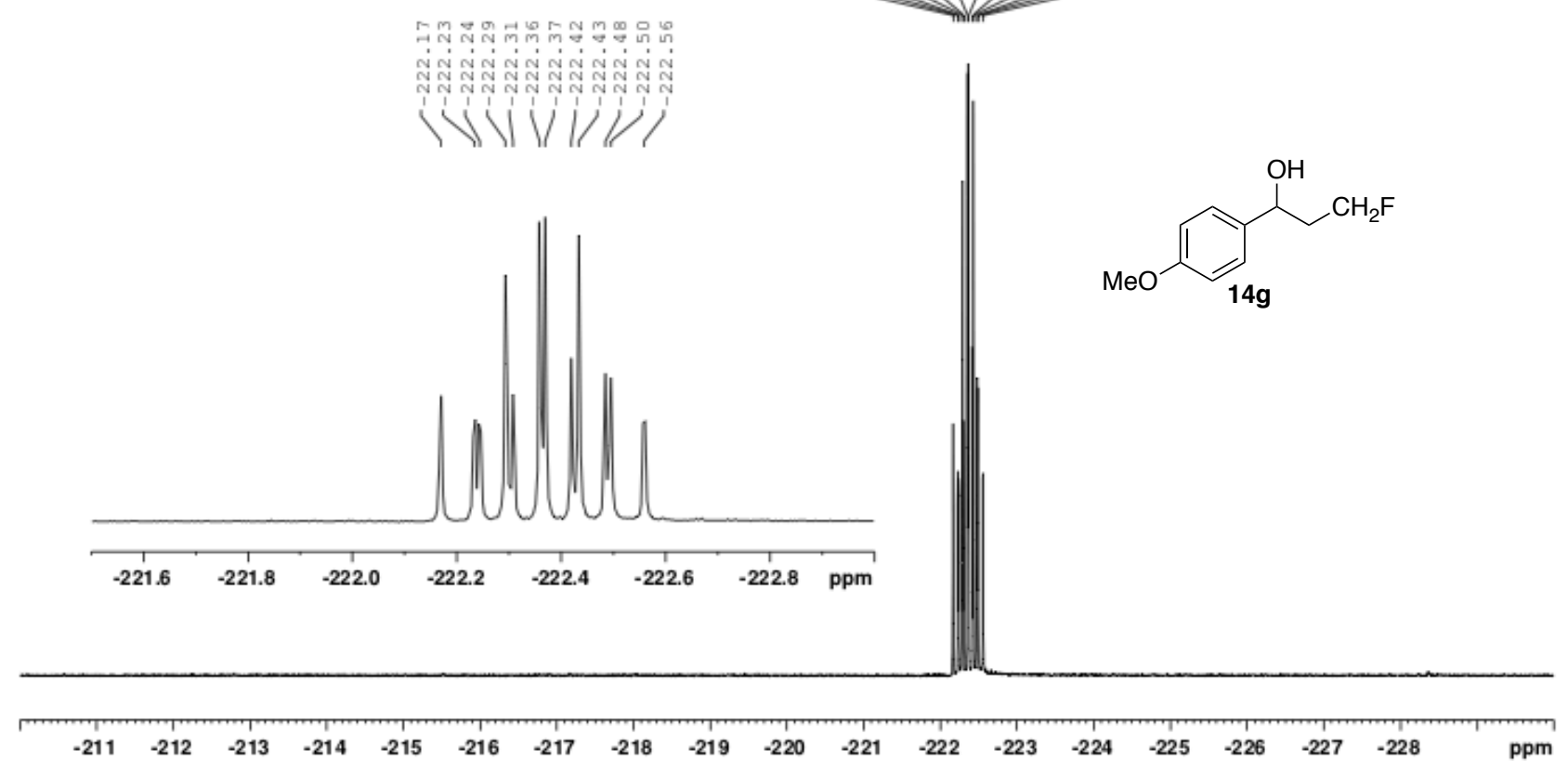

${ }^{1} \mathrm{H}$ NMR $\left(400 \mathrm{MHz}, \mathrm{CDCl}_{3}, \mathrm{rt}\right)$<smiles>COc1ccc(C(O)CCF)cc1</smiles>

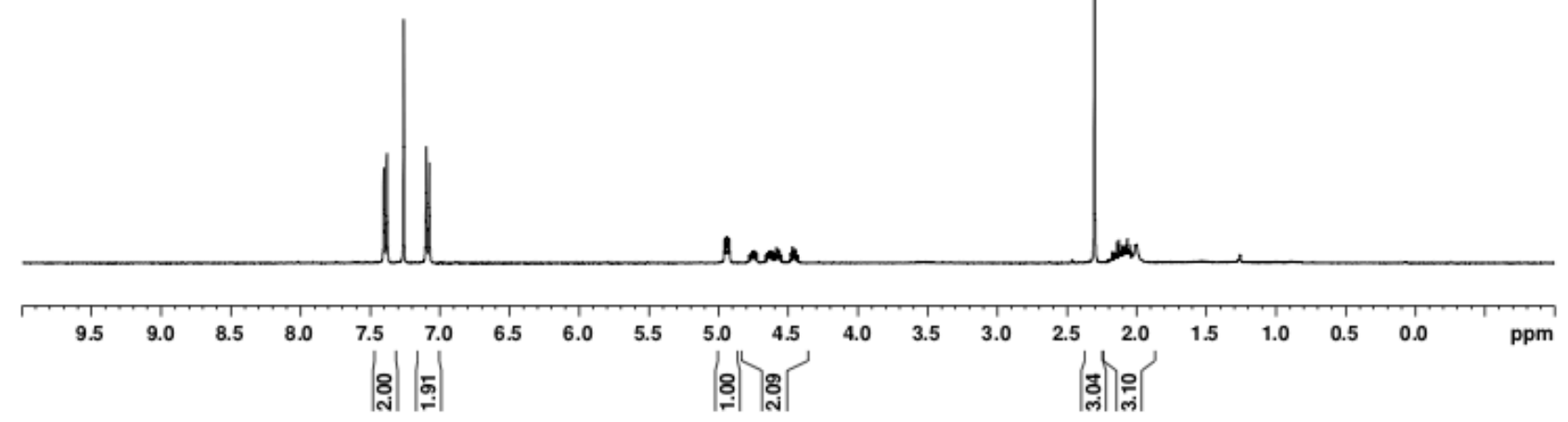


${ }^{13} \mathrm{C}$ NMR $\left(125 \mathrm{MHz}, \mathrm{CDCl}_{3}, \mathrm{rt}\right)$

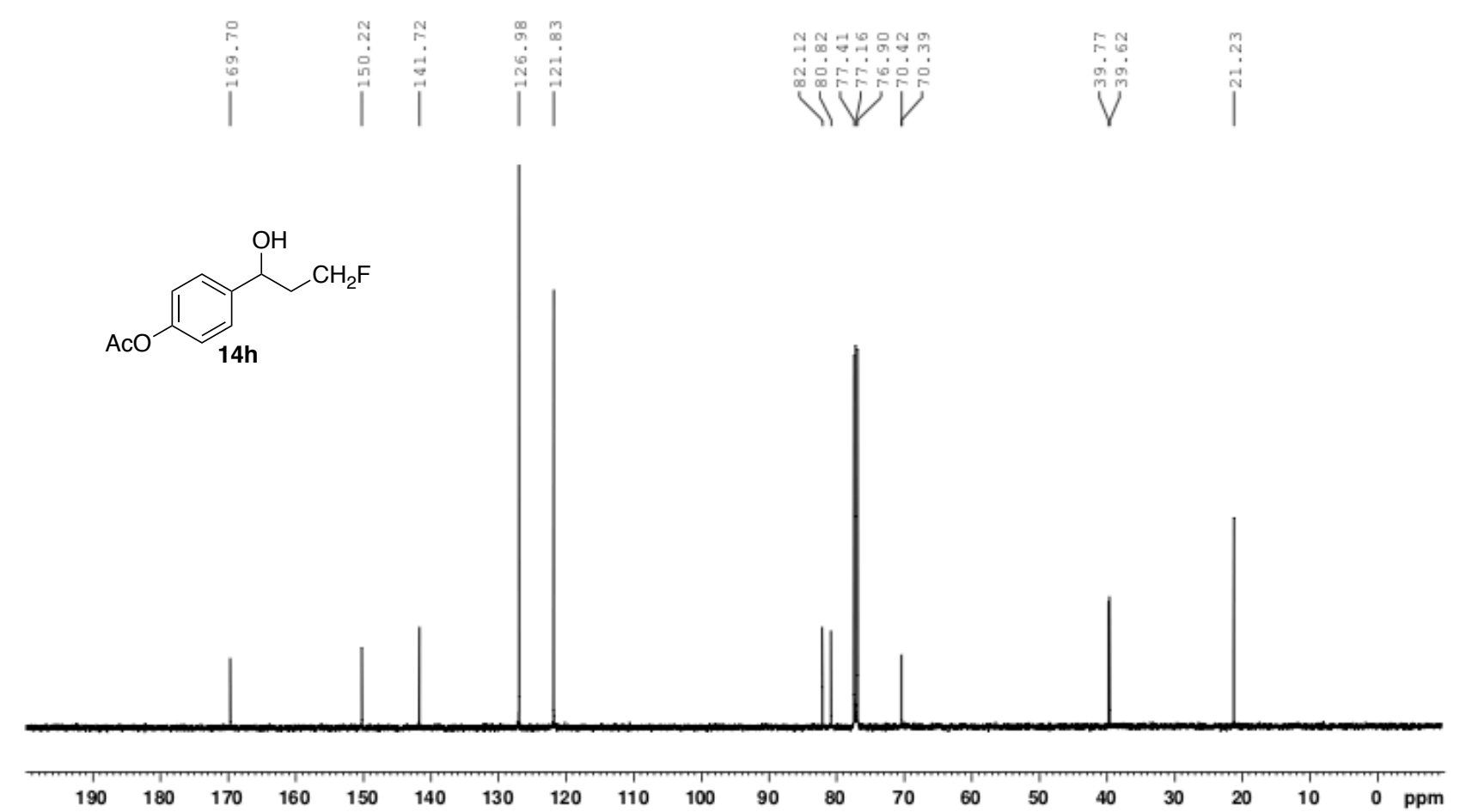

${ }^{19} \mathrm{~F} \mathrm{NMR}\left(376 \mathrm{MHz}, \mathrm{CDCl}_{3}, \mathrm{rt}\right)$

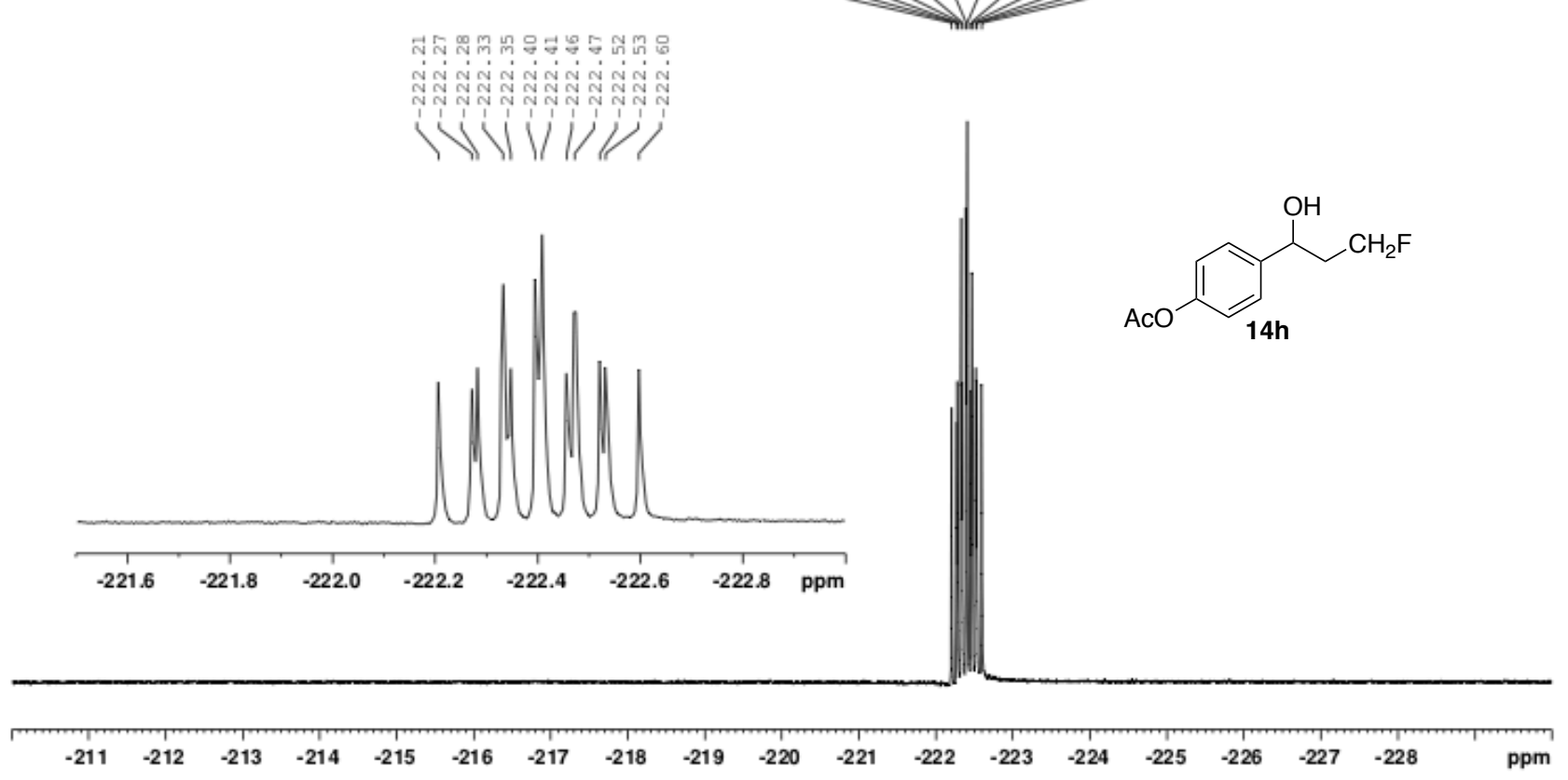


${ }^{1} \mathrm{H}$ NMR $\left(400 \mathrm{MHz}, \mathrm{CDCl}_{3}, \mathrm{rt}\right)$

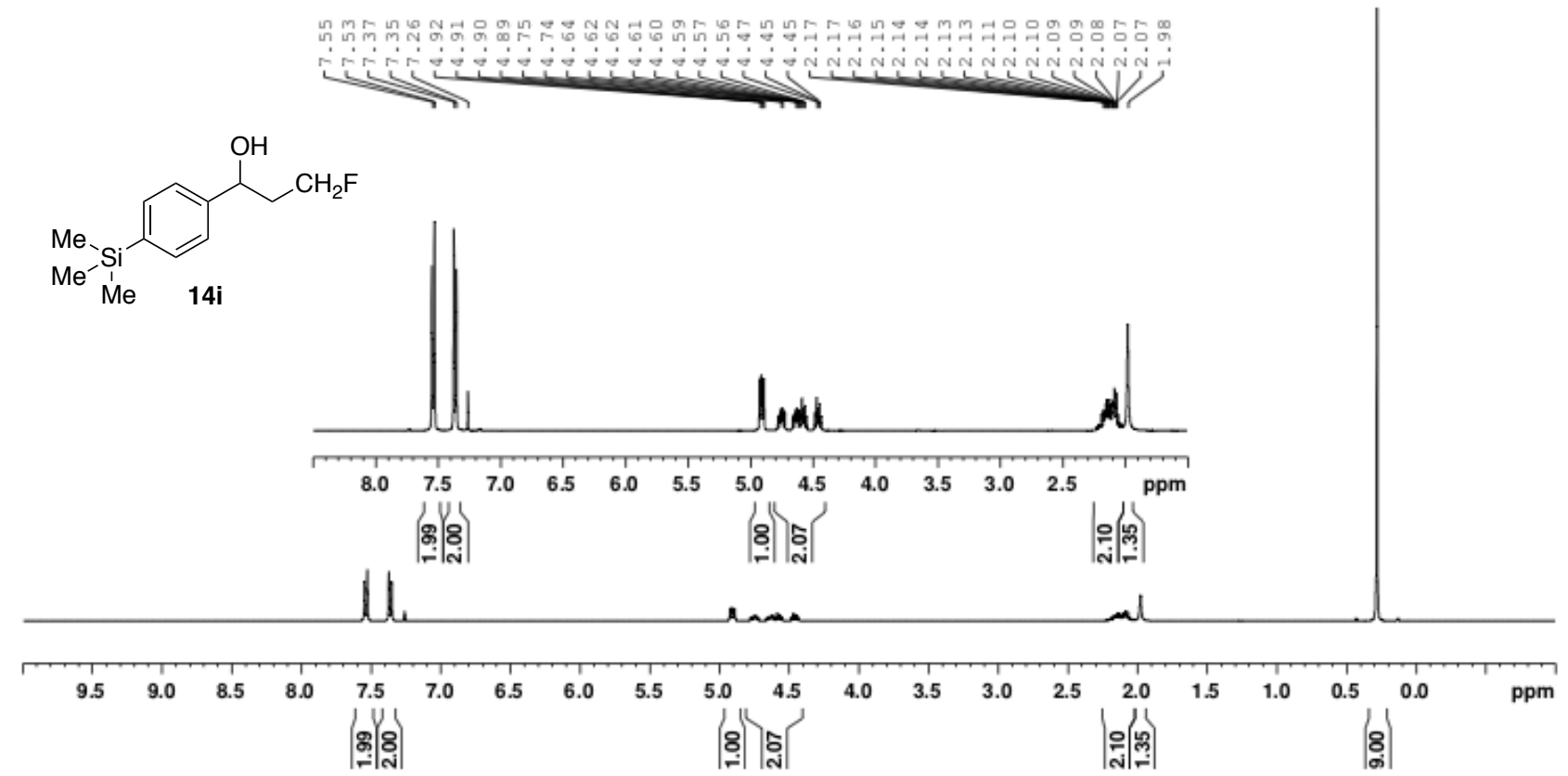

${ }^{13} \mathrm{C}$ NMR $\left(125 \mathrm{MHz}, \mathrm{CDCl}_{3}, \mathrm{rt}\right)$
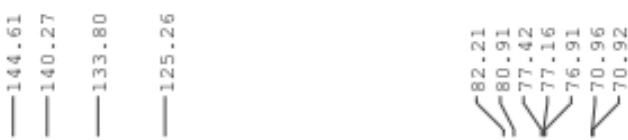<smiles>C[As](C)(C)c1ccc(C(O)CF)cc1</smiles>

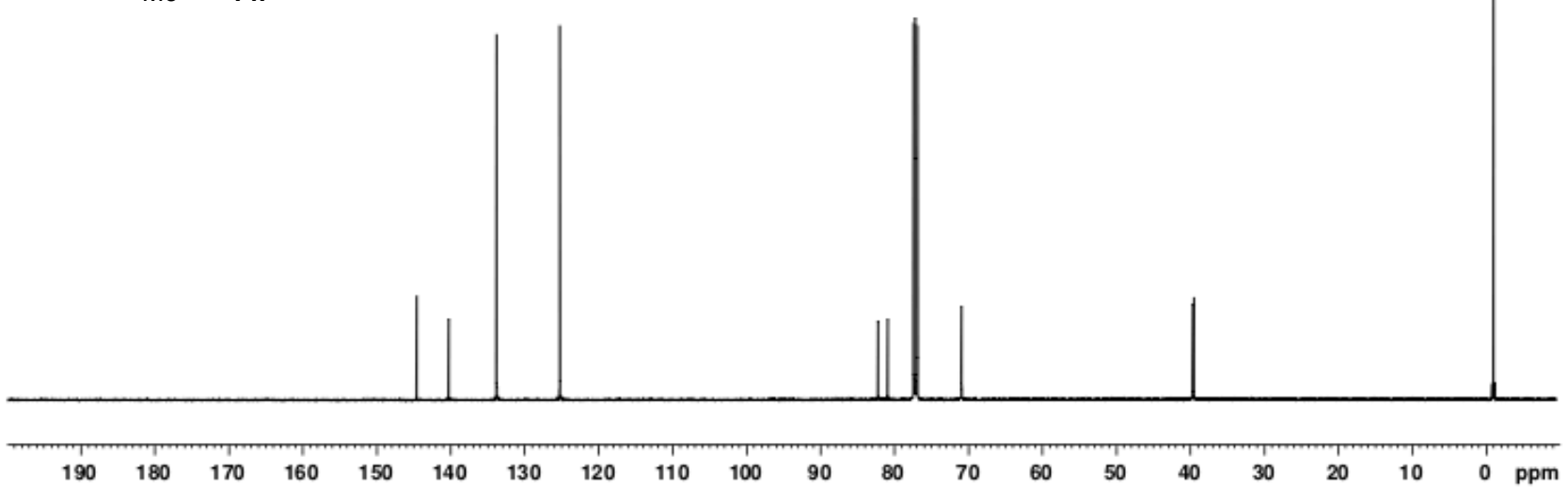


${ }^{19} \mathrm{~F} \mathrm{NMR}\left(376 \mathrm{MHz}, \mathrm{CDCl}_{3}, \mathrm{rt}\right)$

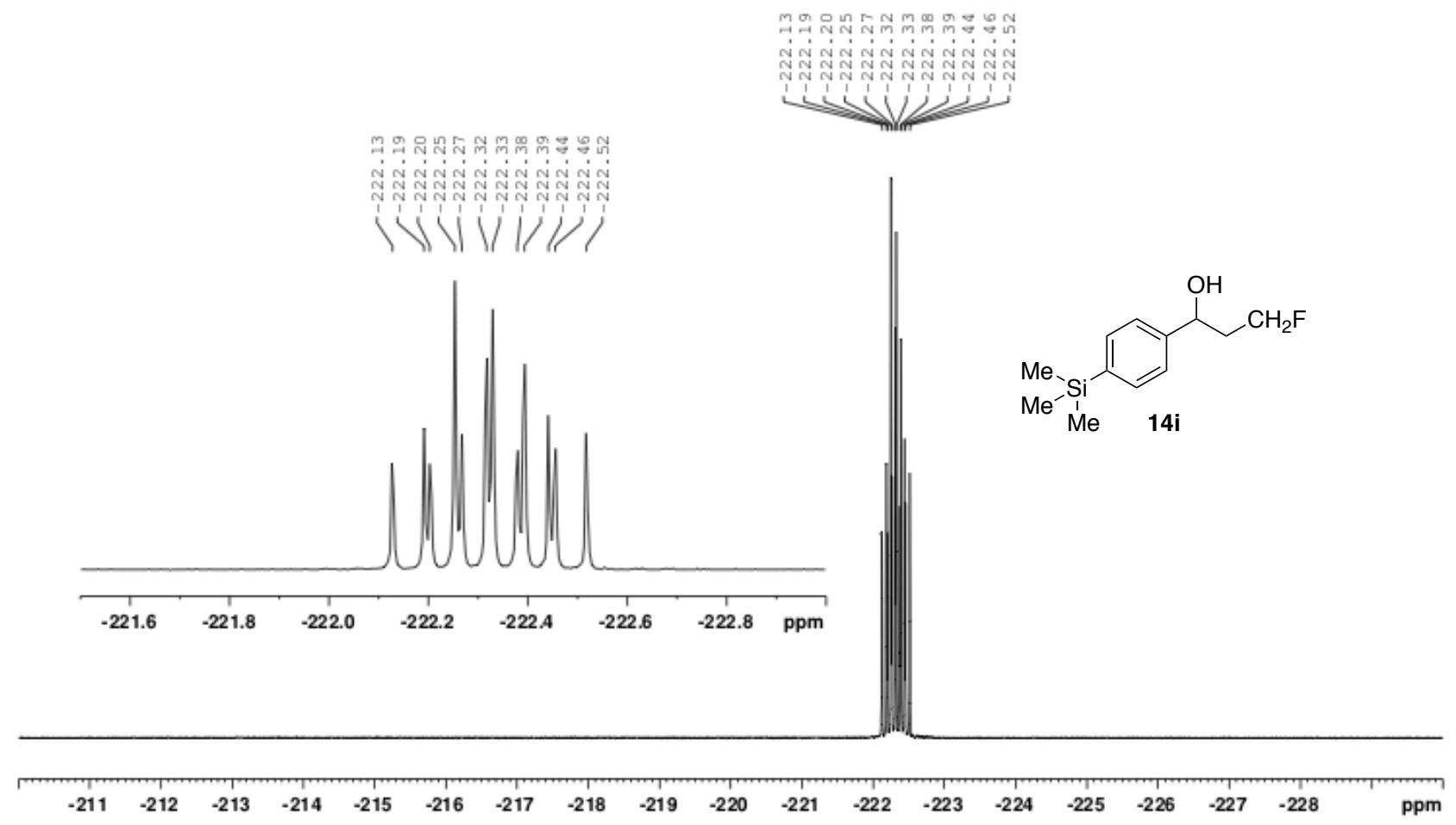

${ }^{1} \mathrm{H}$ NMR $\left(400 \mathrm{MHz}, \mathrm{CDCl}_{3}, \mathrm{rt}\right)$

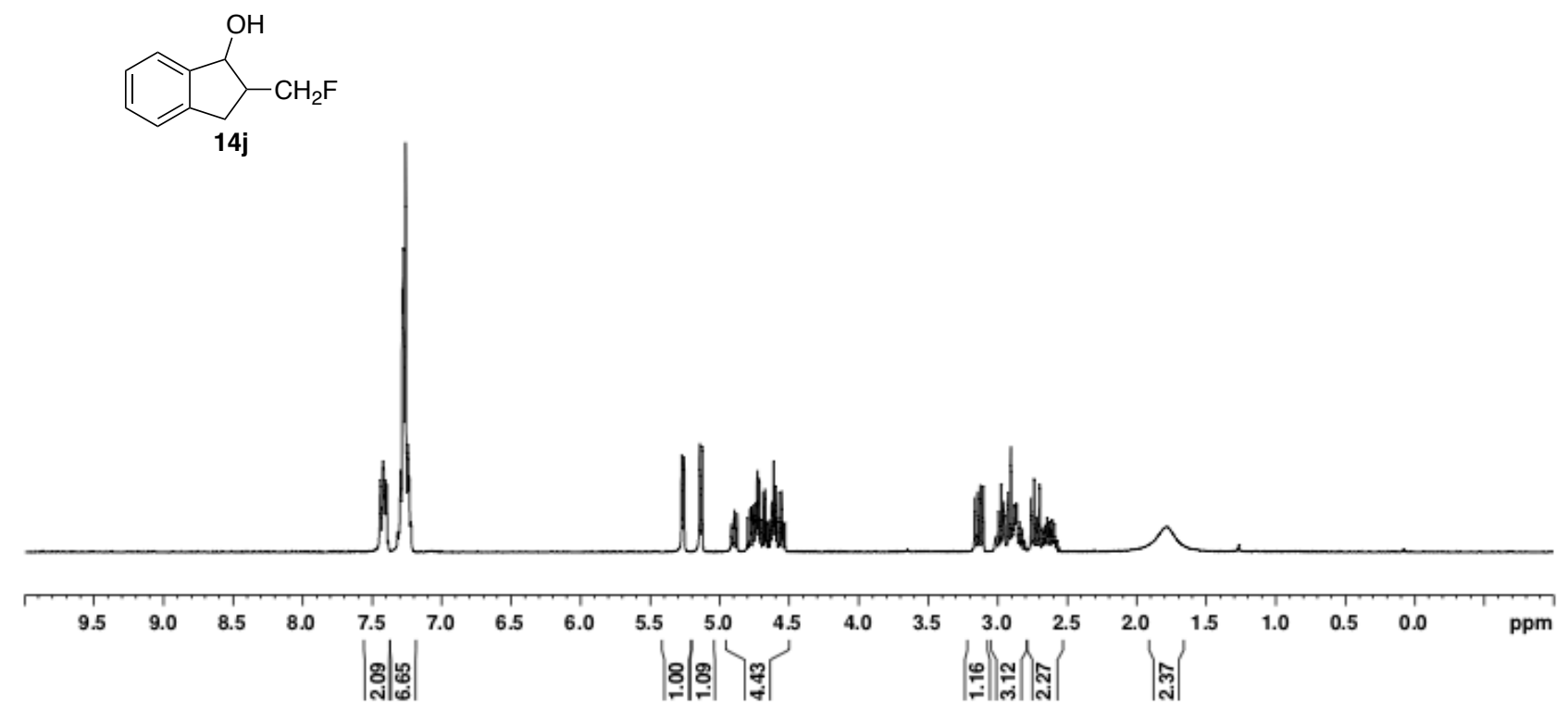


${ }^{13} \mathrm{C}$ NMR $\left(125 \mathrm{MHz}, \mathrm{CDCl}_{3}, \mathrm{rt}\right)$

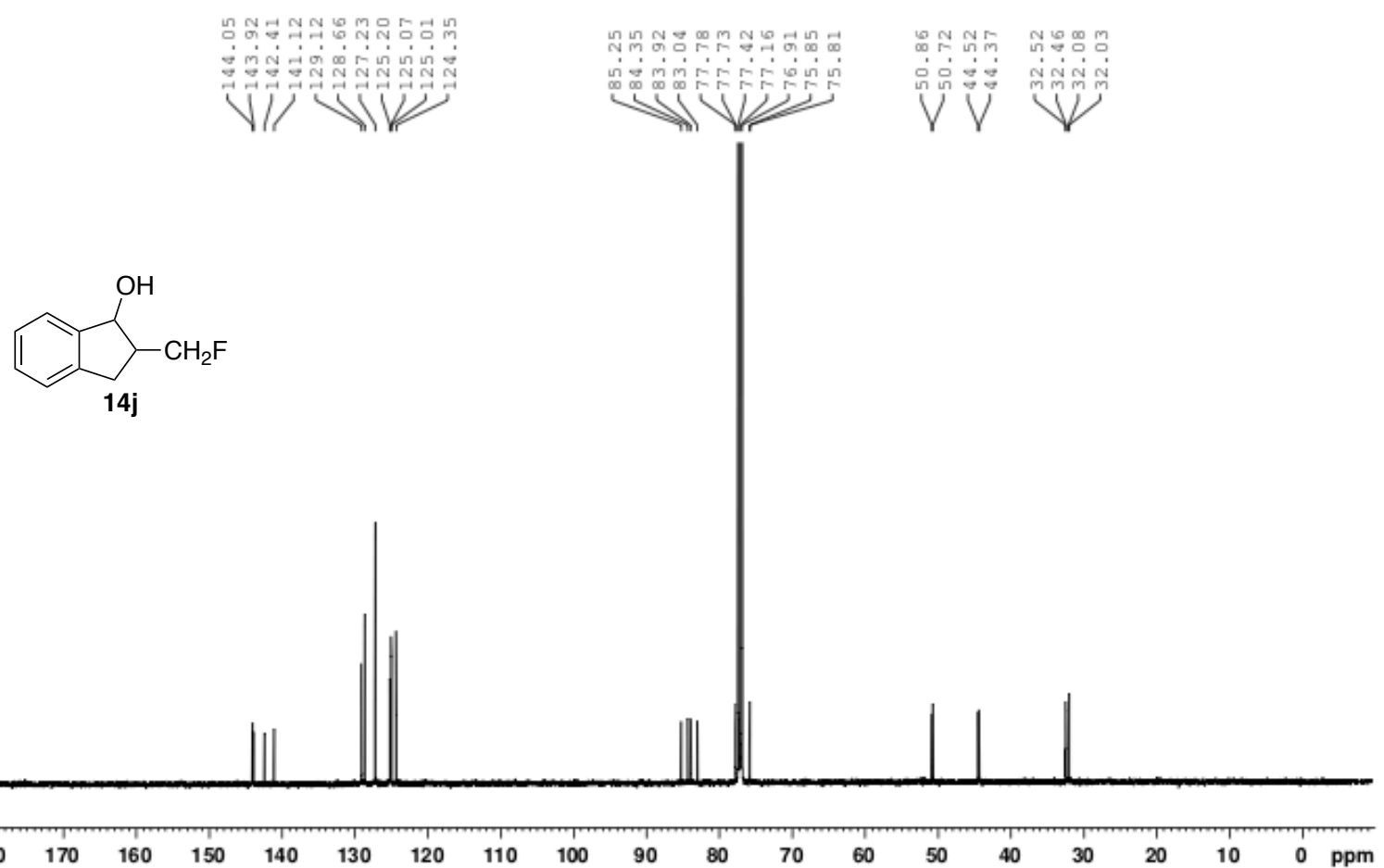

${ }^{19} \mathrm{~F} \mathrm{NMR}\left(376 \mathrm{MHz}, \mathrm{CDCl}_{3}, \mathrm{rt}\right)$
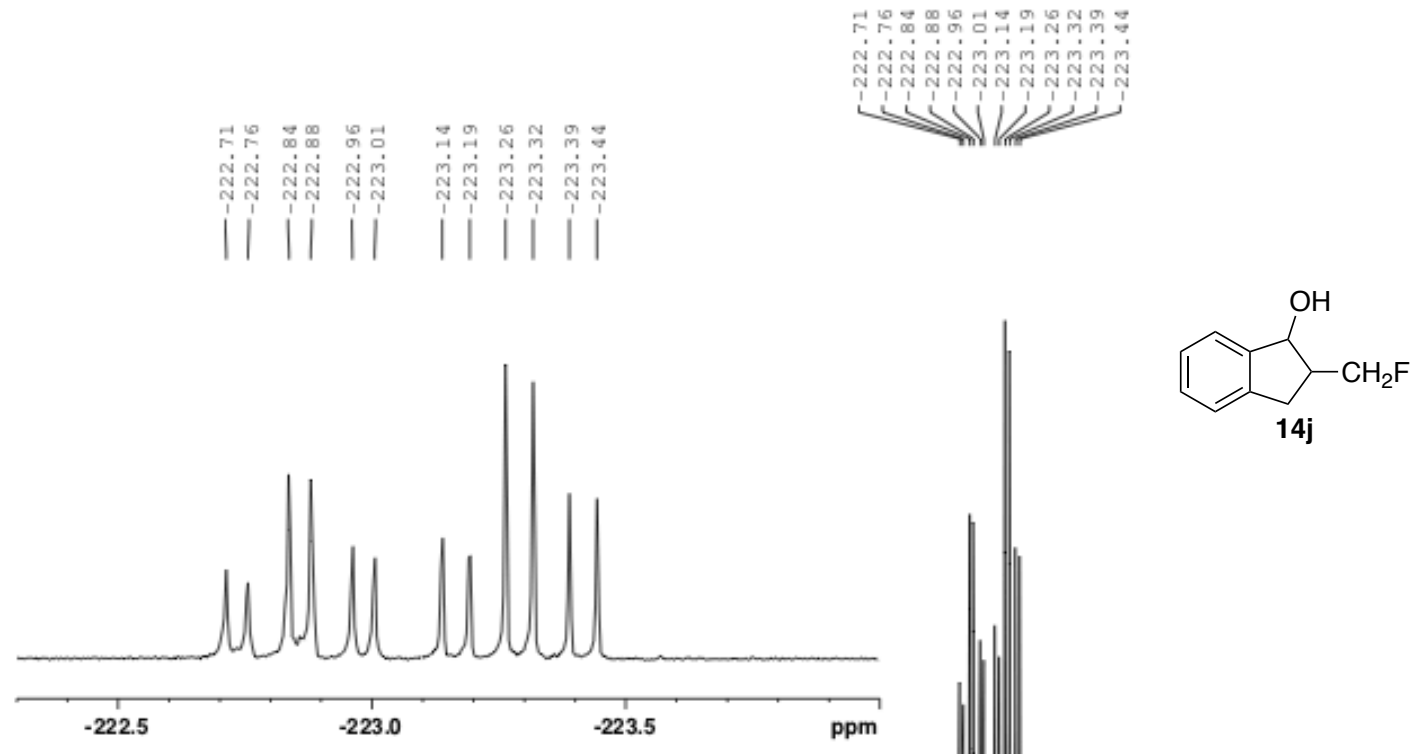

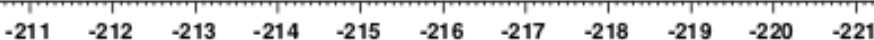

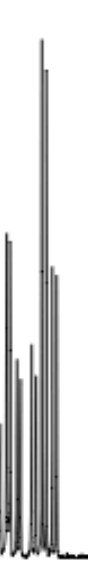

$14 \mathbf{j}$ 
${ }^{1} \mathrm{H} \mathrm{NMR}\left(400 \mathrm{MHz}, \mathrm{CDCl}_{3}, \mathrm{rt}\right)$<smiles>Cc1cccc(C(O)CCF)c1</smiles>
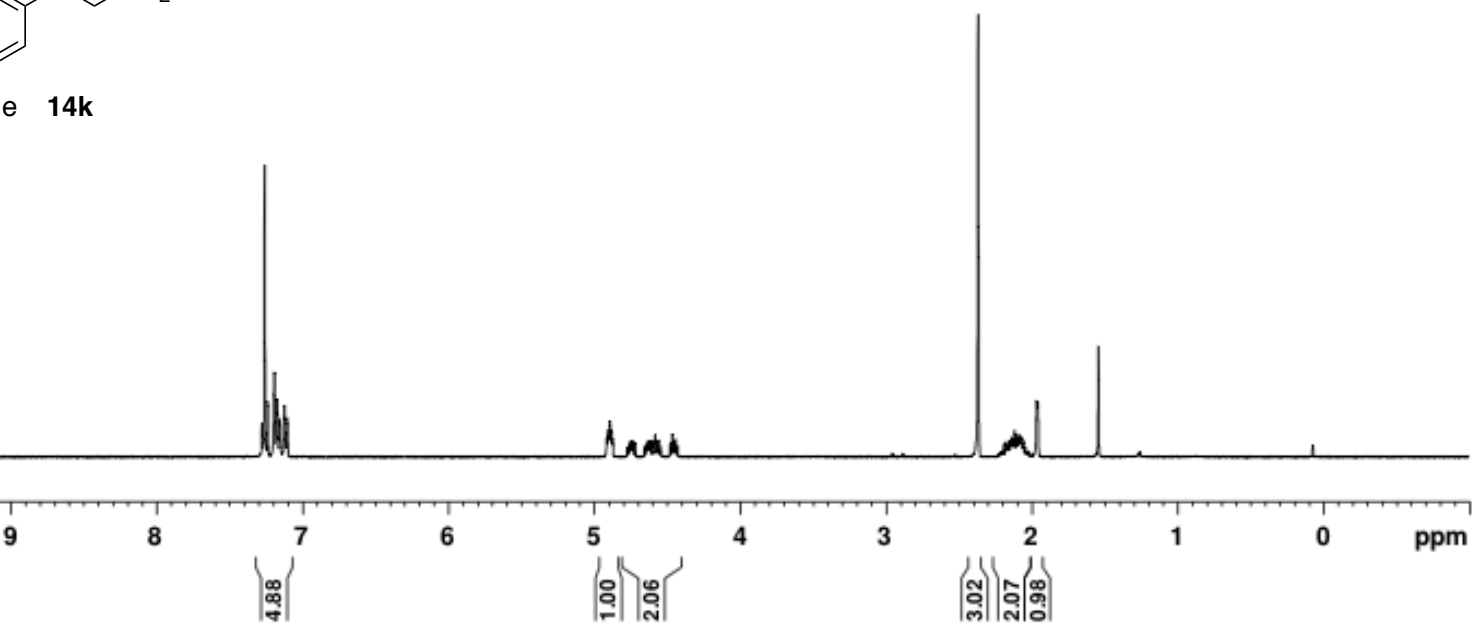

${ }^{13} \mathrm{C}$ NMR $\left(125 \mathrm{MHz}, \mathrm{CDCl}_{3}, \mathrm{rt}\right)$
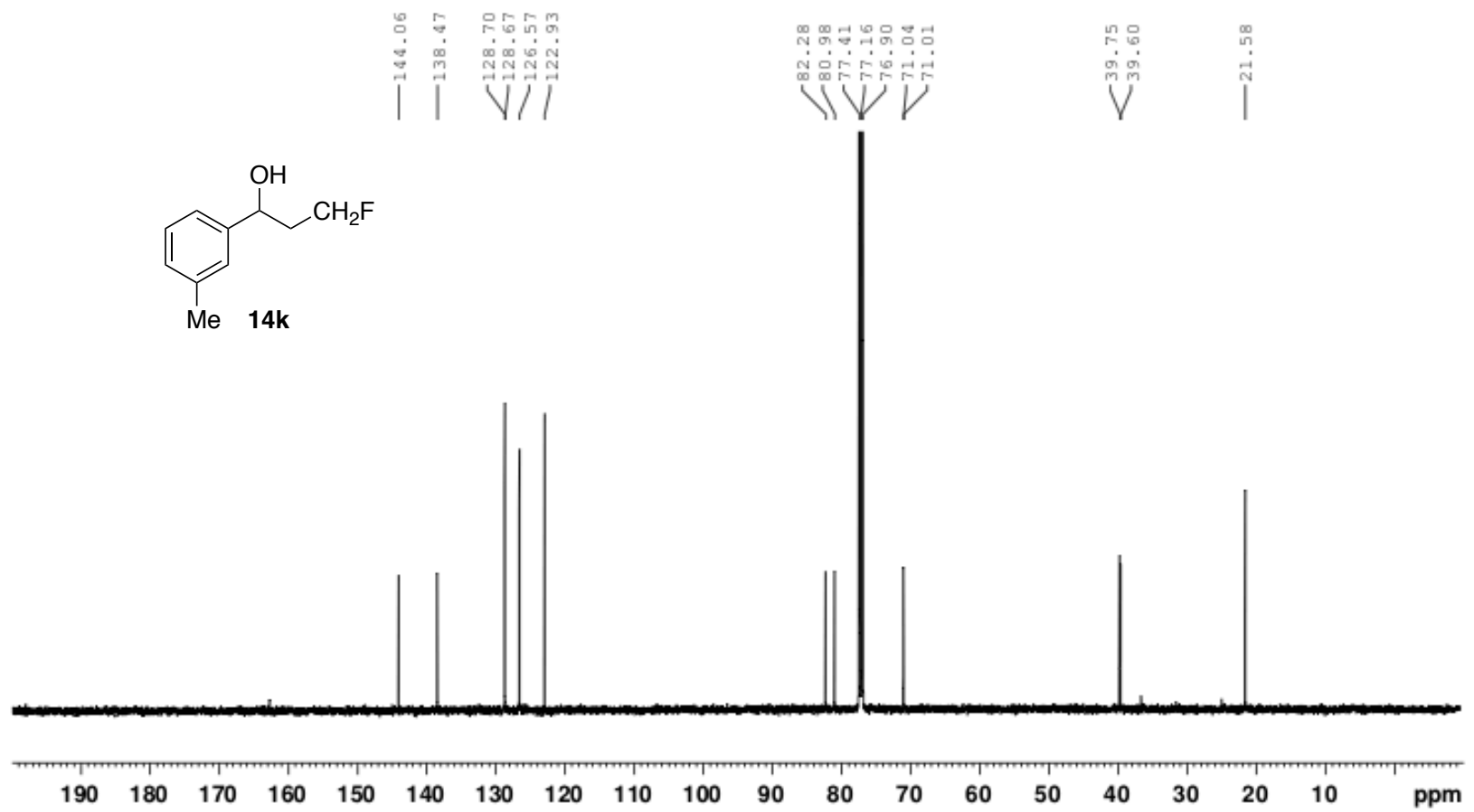
${ }^{19} \mathrm{~F} \mathrm{NMR}\left(376 \mathrm{MHz}, \mathrm{CDCl}_{3}, \mathrm{rt}\right)$

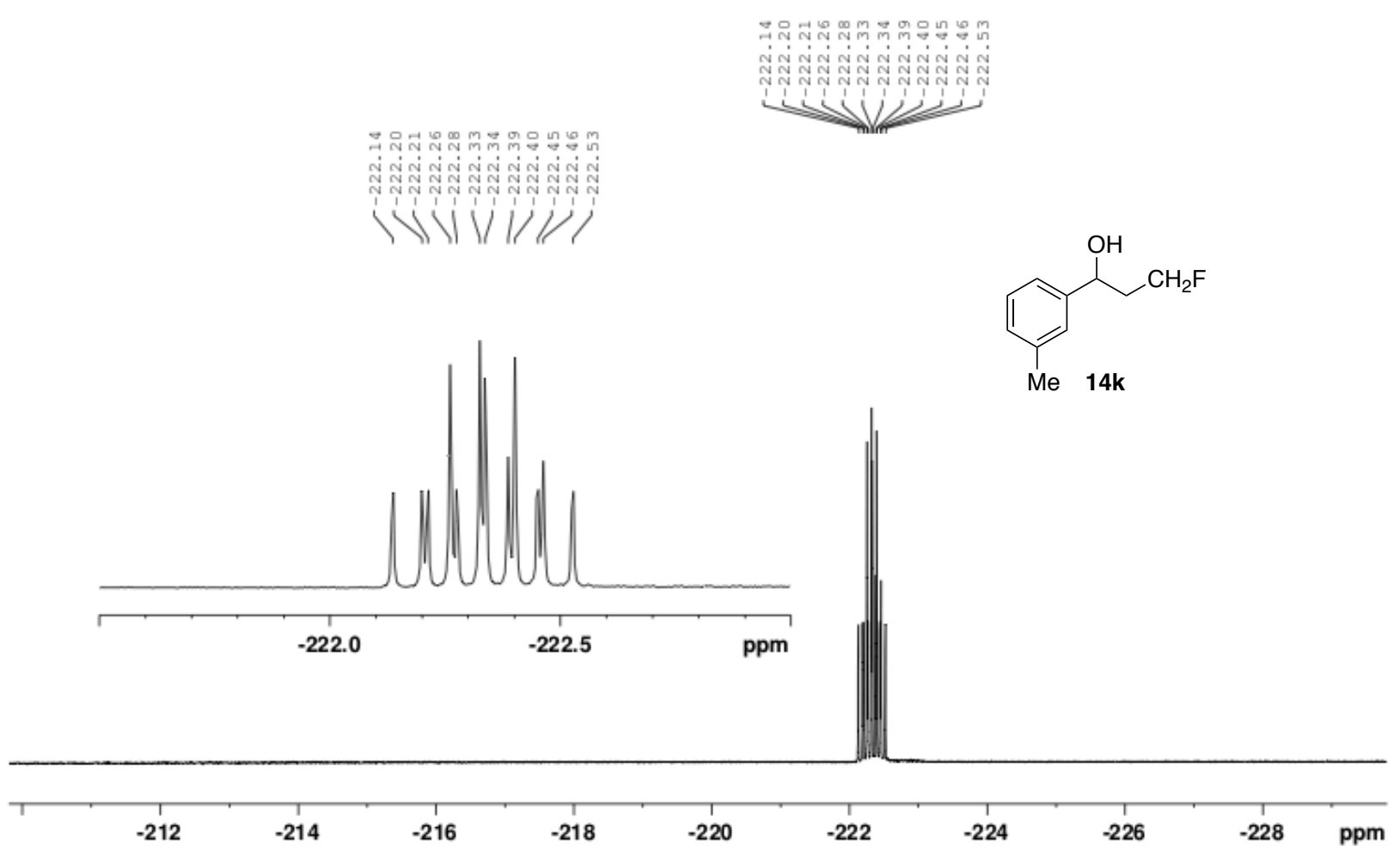

${ }^{1} \mathrm{H} \mathrm{NMR}\left(400 \mathrm{MHz}, \mathrm{CDCl}_{3}, \mathrm{rt}\right)$

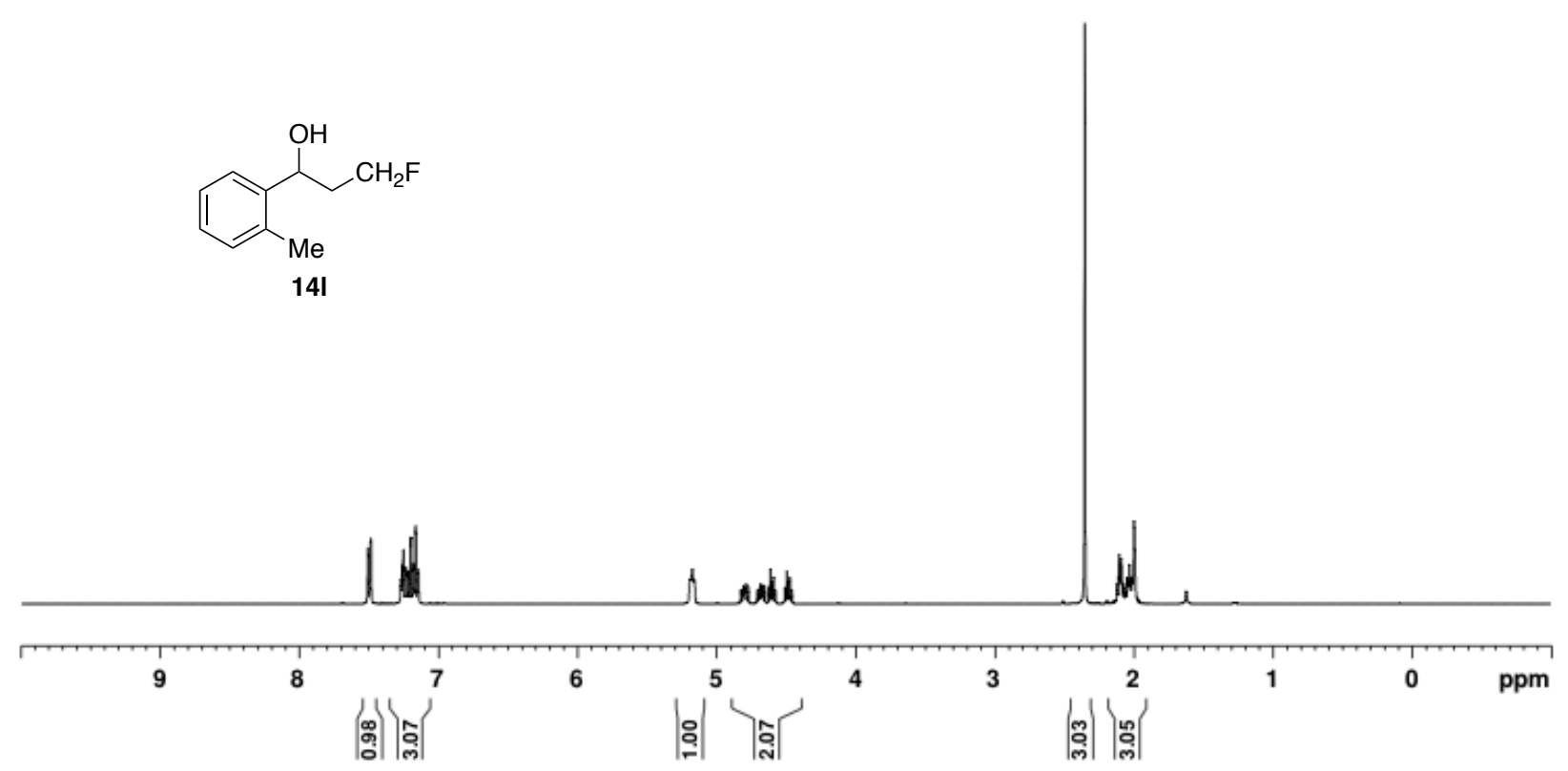


${ }^{13} \mathrm{C}$ NMR $\left(125 \mathrm{MHz}, \mathrm{CDCl}_{3}, \mathrm{rt}\right)$

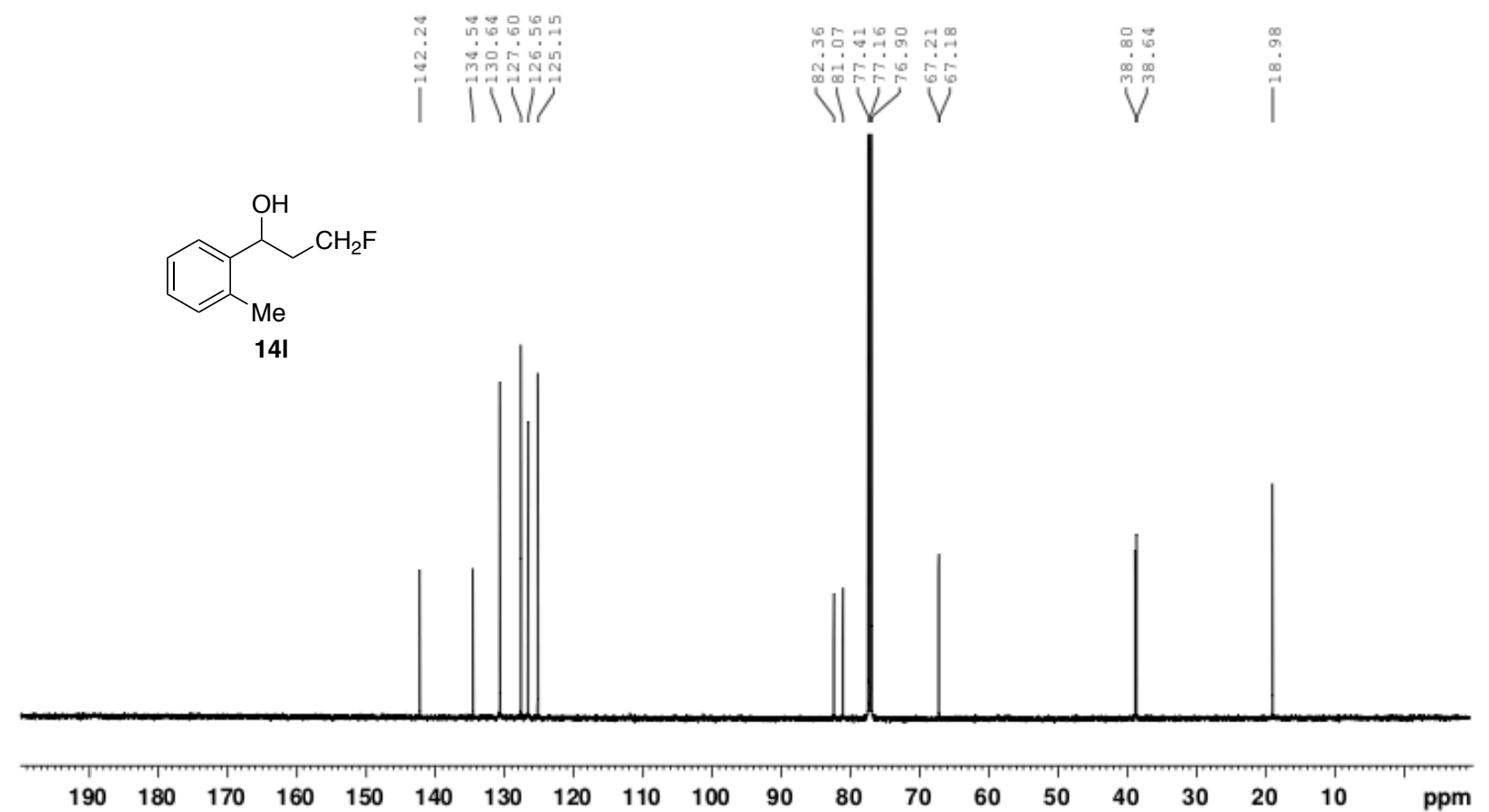

${ }^{19} \mathrm{~F} \mathrm{NMR}\left(376 \mathrm{MHz}, \mathrm{CDCl}_{3}, \mathrm{rt}\right)$
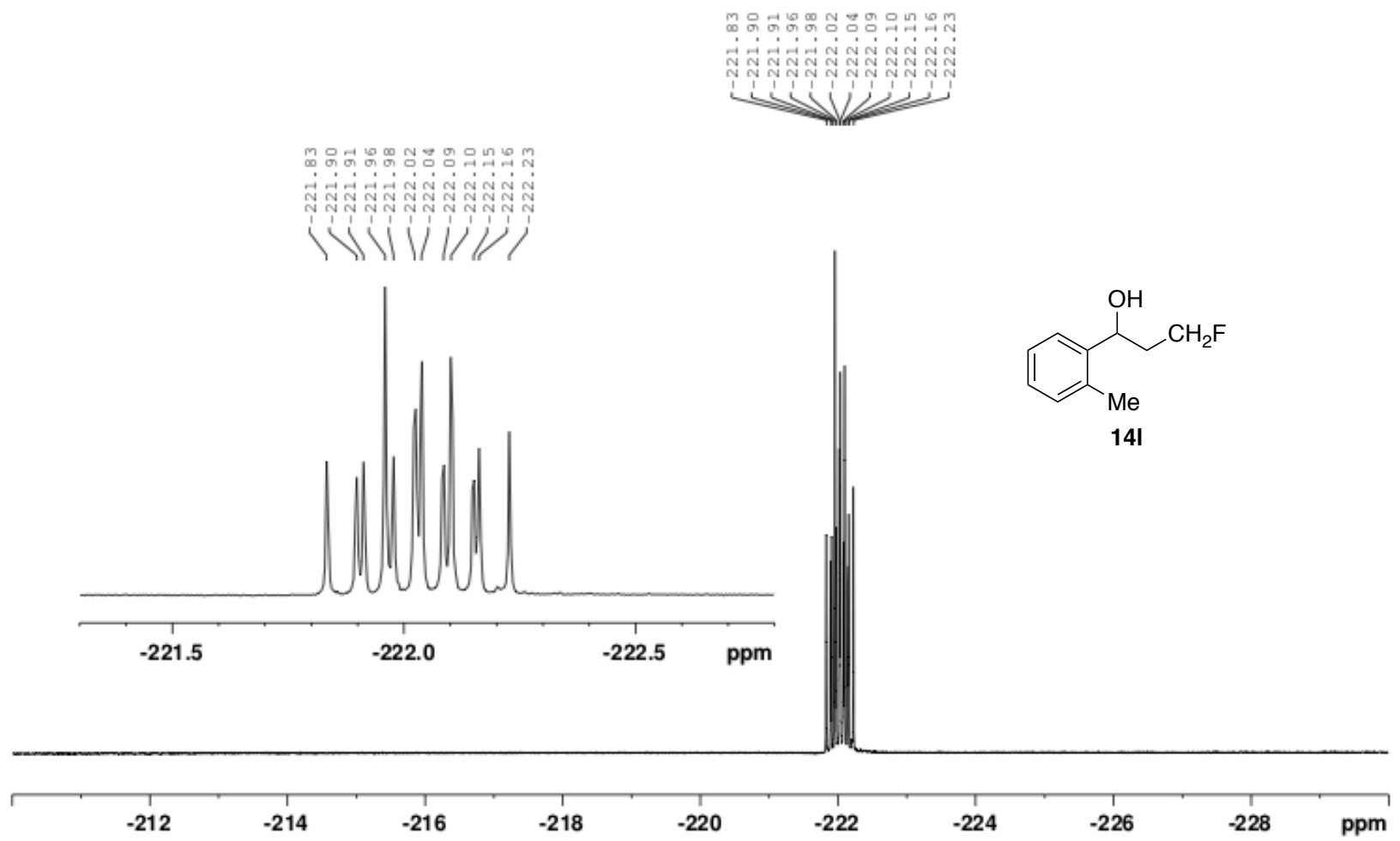
${ }^{1} \mathrm{H}$ NMR $\left(400 \mathrm{MHz}, \mathrm{CDCl}_{3}, \mathrm{rt}\right)$

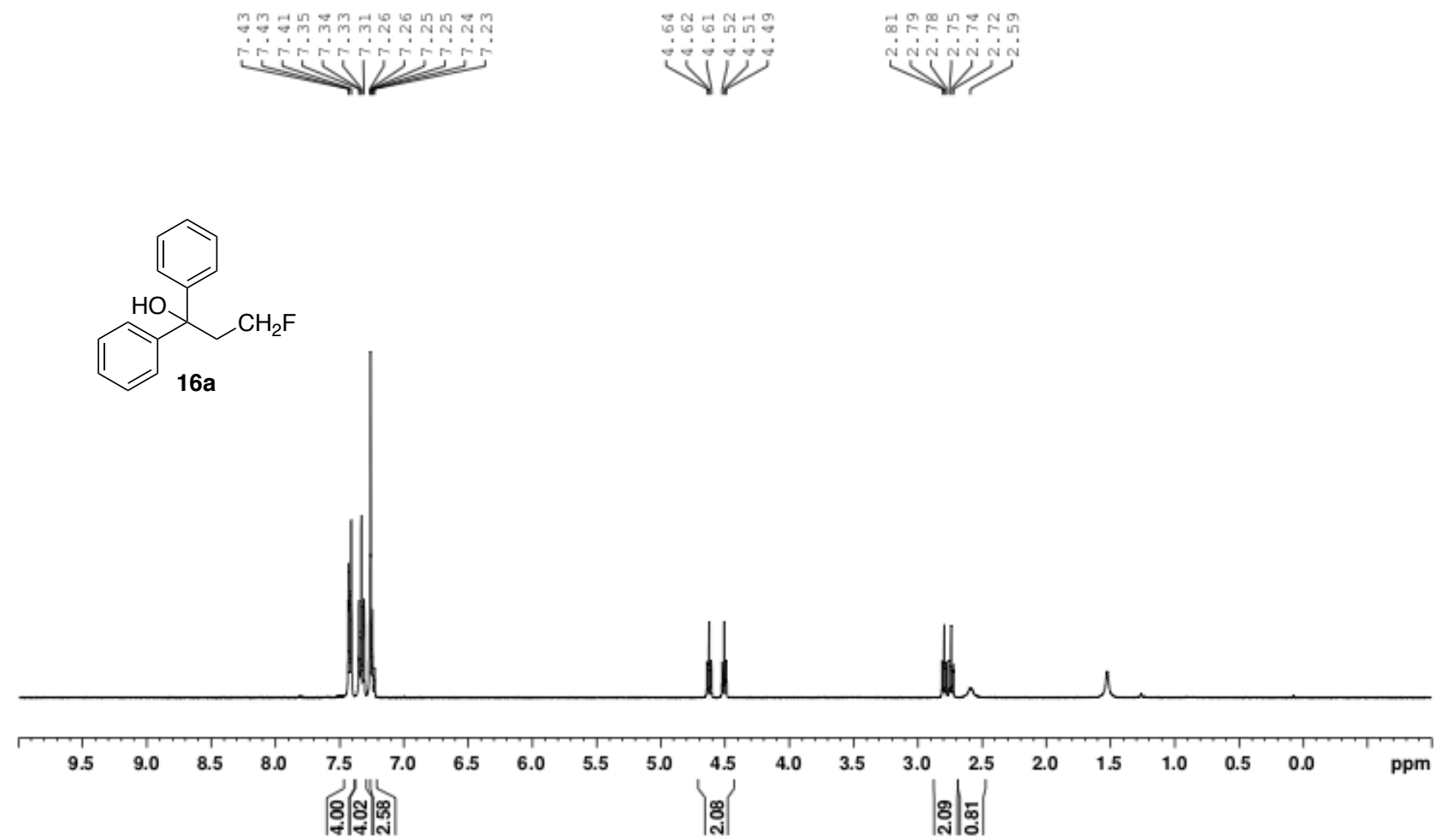

${ }^{13} \mathrm{C}$ NMR (125 MHz, $\left.\mathrm{CDCl}_{3}, \mathrm{rt}\right)$
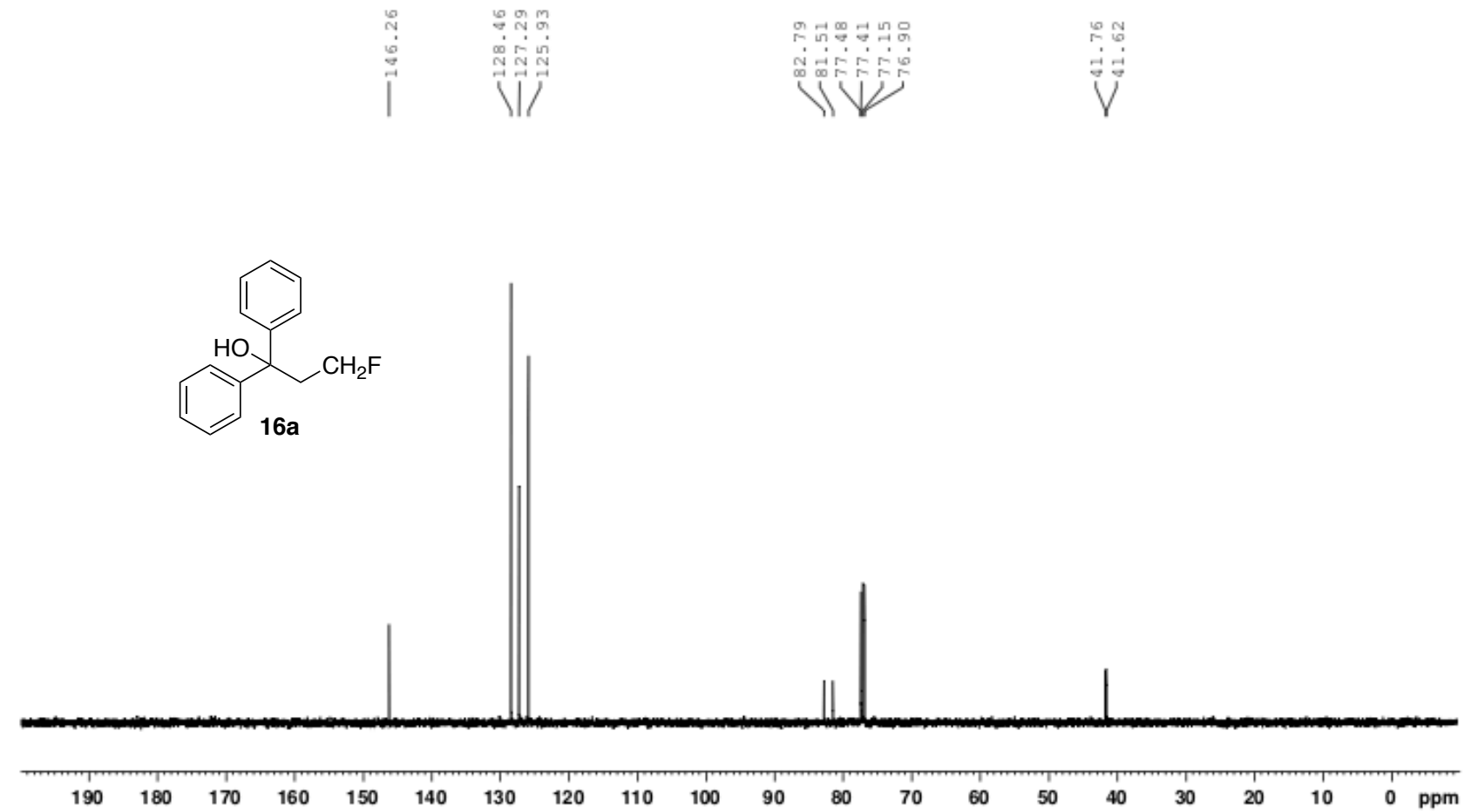
${ }^{19} \mathrm{~F} \mathrm{NMR}\left(376 \mathrm{MHz}, \mathrm{CDCl}_{3}, \mathrm{rt}\right)$

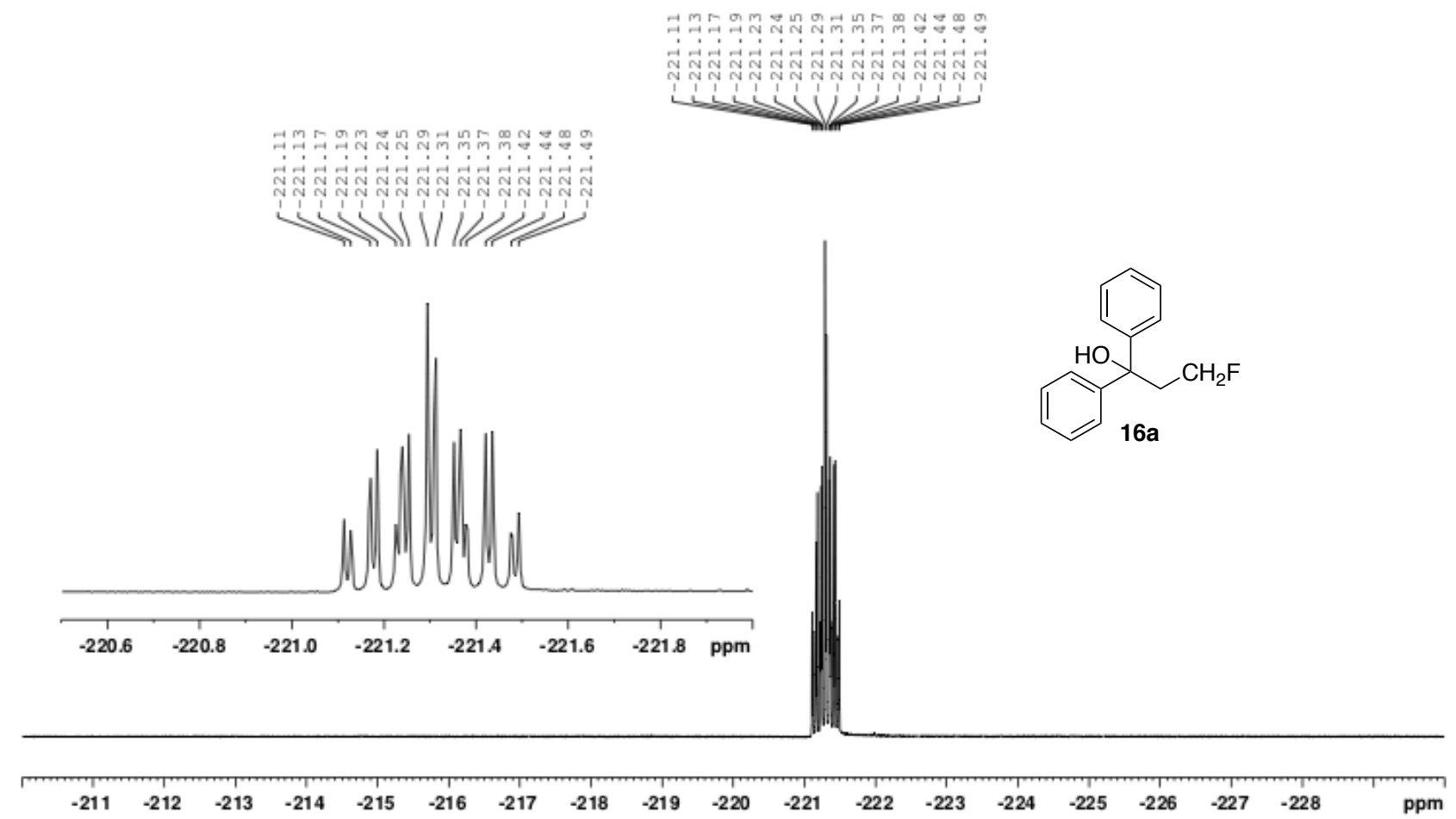

${ }^{1} \mathrm{H}$ NMR (400 MHz, CDCl 3 , rt)
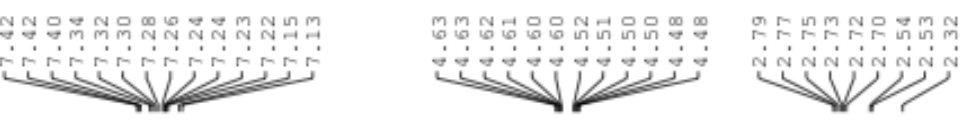<smiles>Cc1ccc(C(CCF)(CCF)c2ccccc2)cc1</smiles>

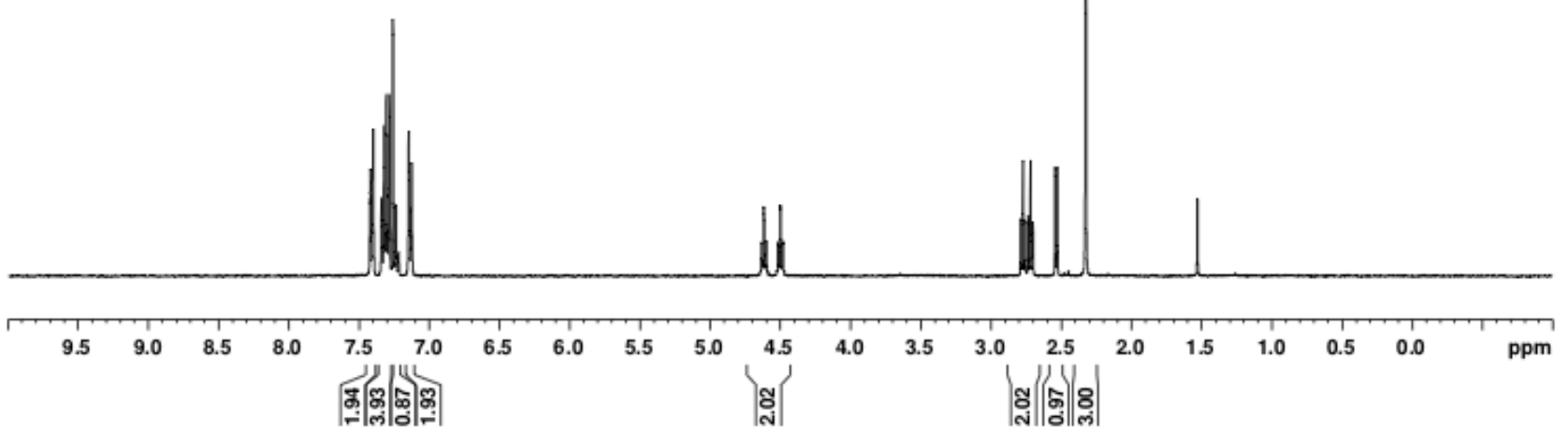


${ }^{13} \mathrm{C}$ NMR $\left(125 \mathrm{MHz}, \mathrm{CDCl}_{3}, \mathrm{rt}\right)$

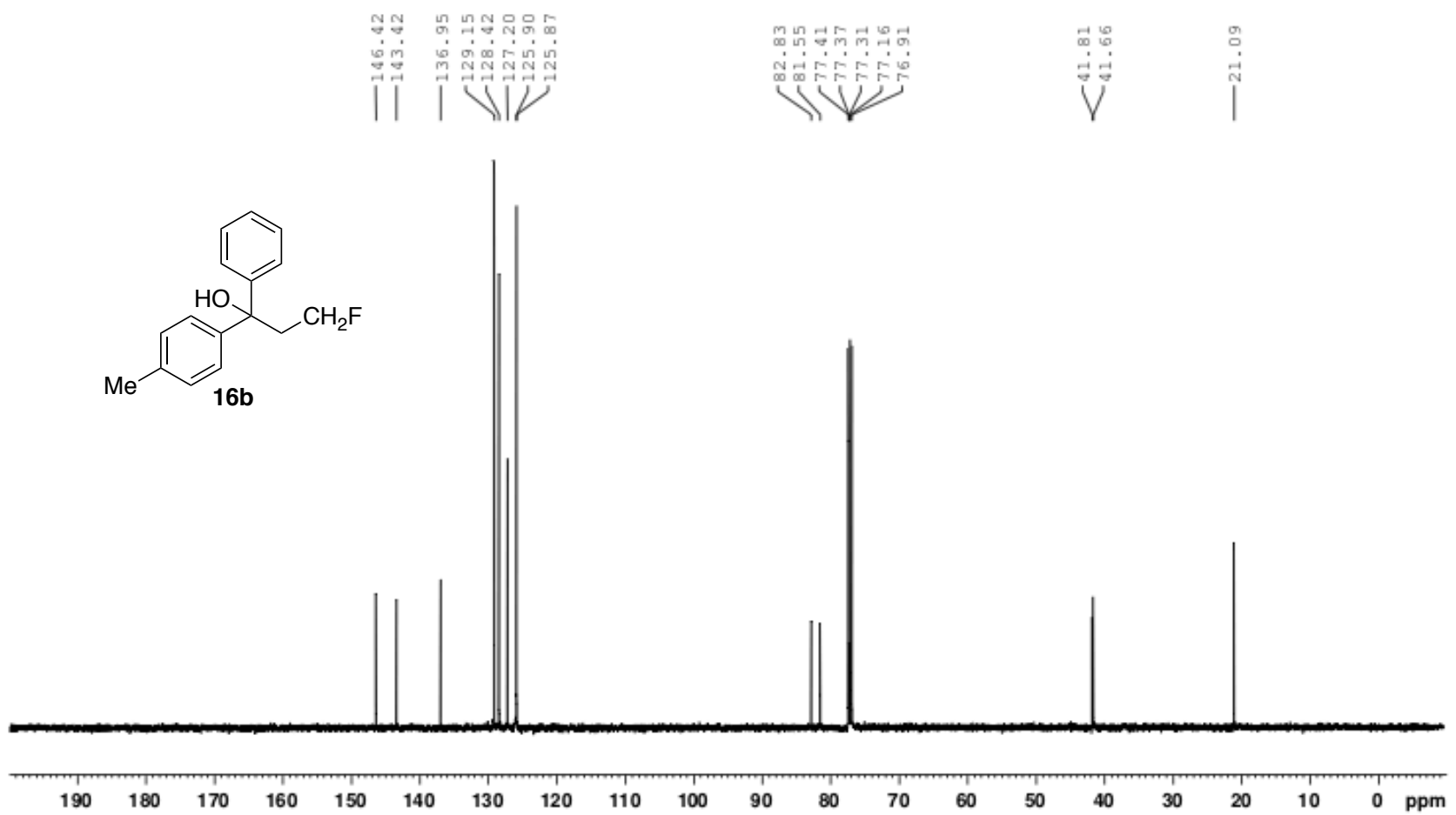

${ }^{19} \mathrm{~F} \mathrm{NMR}\left(376 \mathrm{MHz}, \mathrm{CDCl}_{3}, \mathrm{rt}\right)$

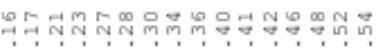

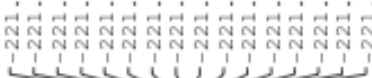

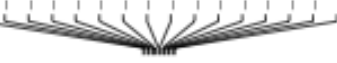

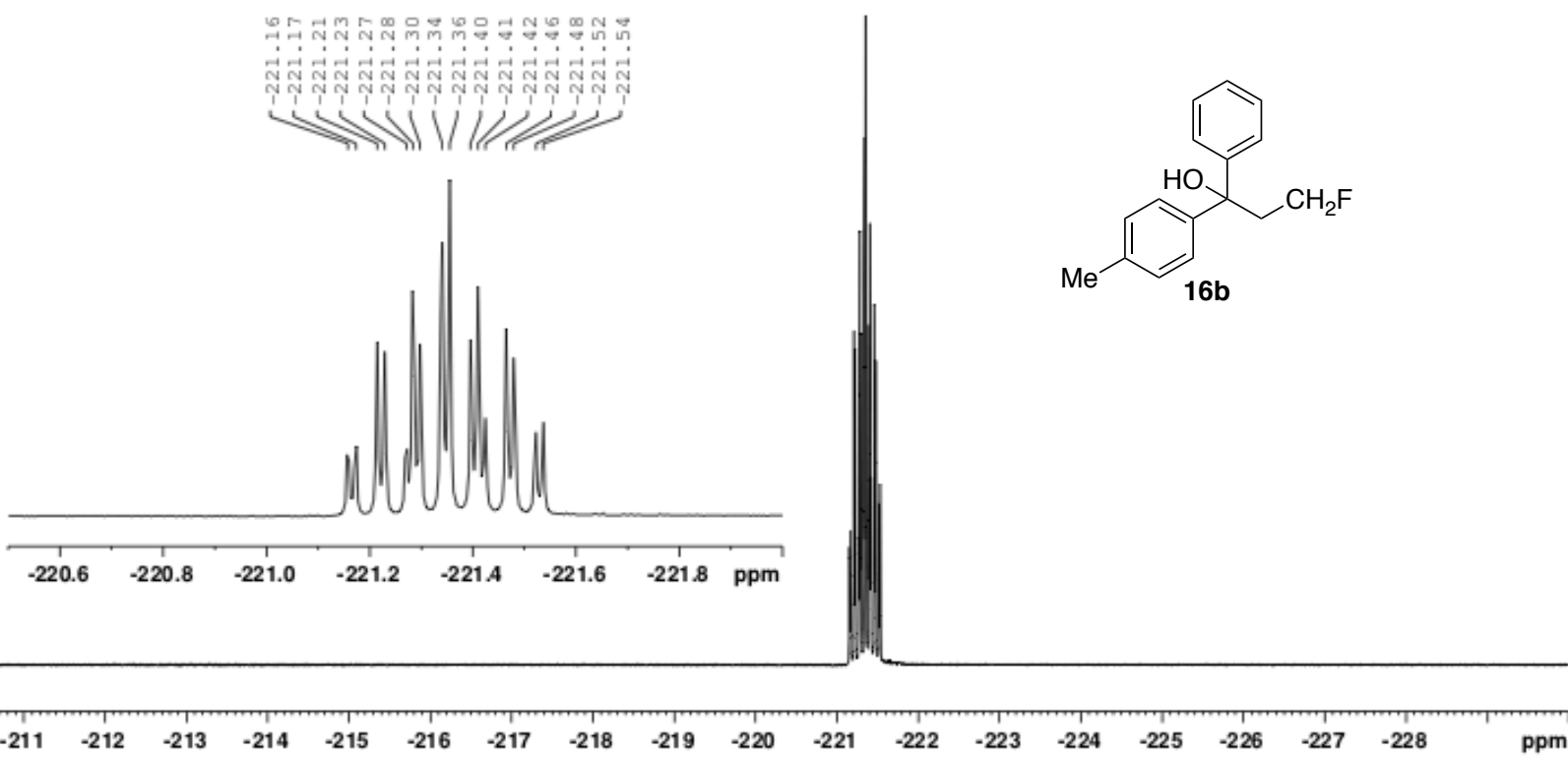


${ }^{1} \mathrm{H}$ NMR $\left(400 \mathrm{MHz}, \mathrm{CDCl}_{3}, \mathrm{rt}\right)$

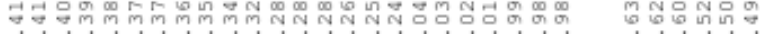

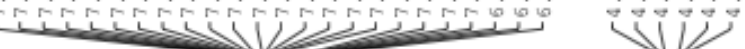

0
0

$\underbrace{\text { Nind }}$<smiles>OC(CCF)(c1ccccc1)c1ccc(F)cc1</smiles>

$16 c$

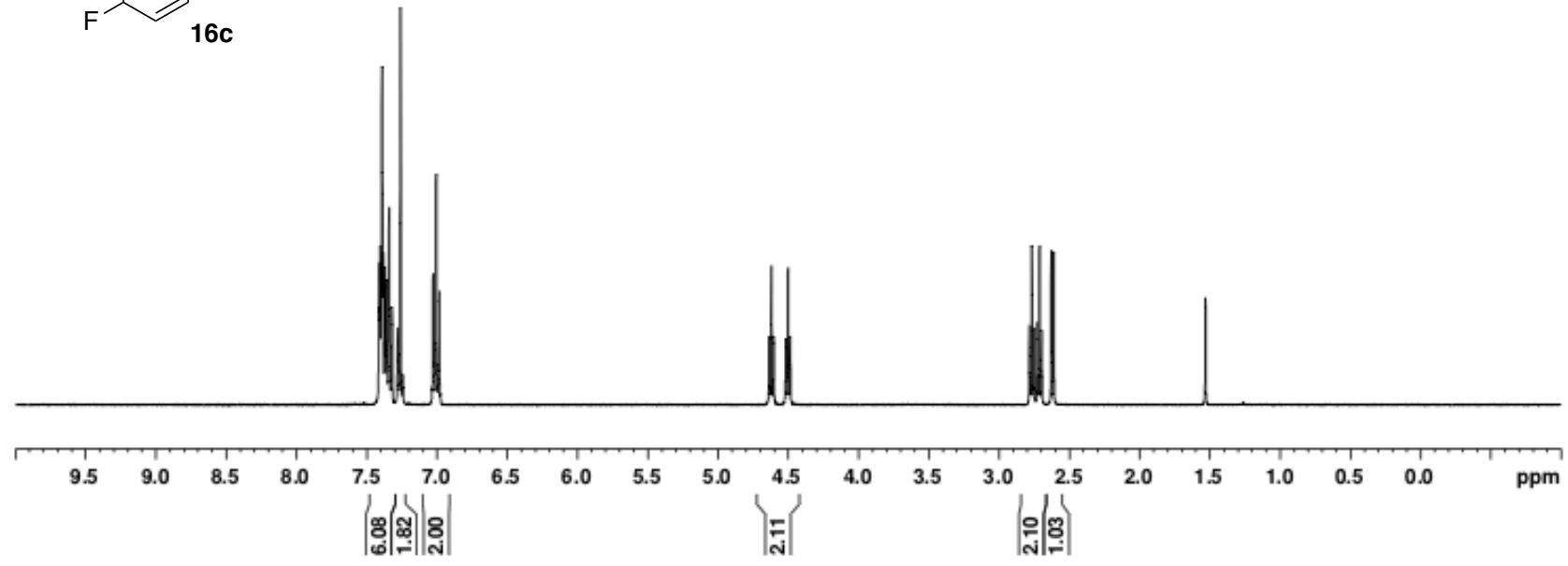

${ }^{13} \mathrm{C}$ NMR $\left(125 \mathrm{MHz}, \mathrm{CDCl}_{3}, \mathrm{rt}\right)$
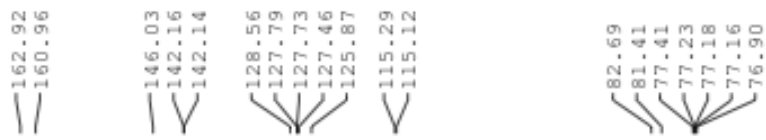<smiles>OC(CCF)(c1ccccc1)c1ccc(F)cc1</smiles>

$16 c$

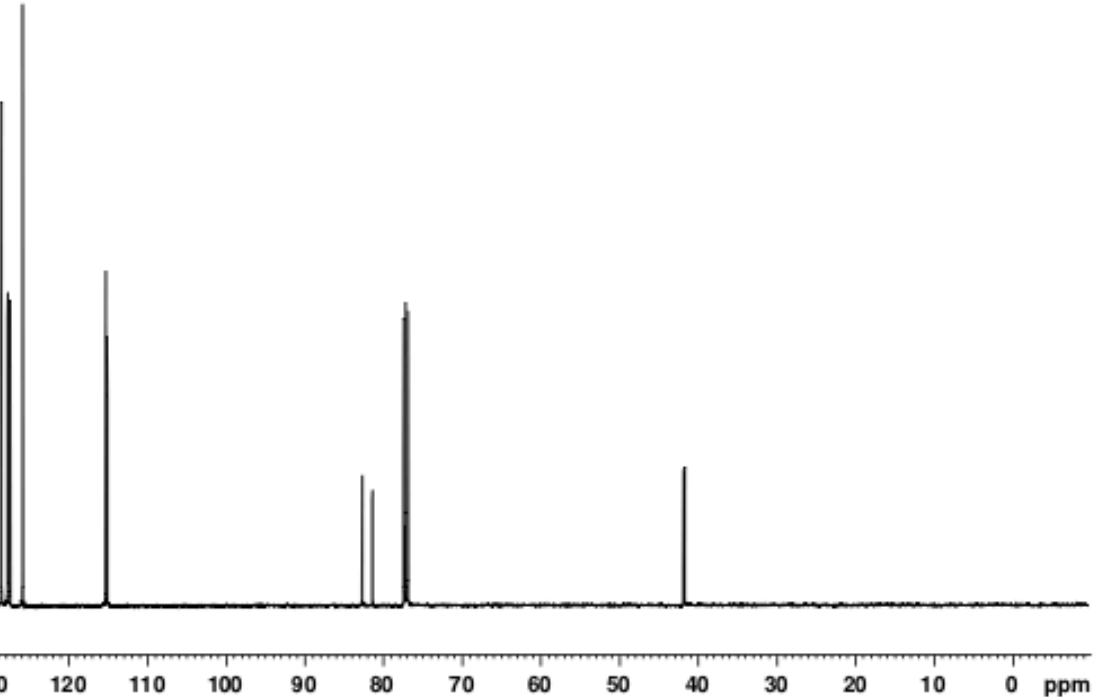


${ }^{19} \mathrm{~F} \mathrm{NMR}\left(376 \mathrm{MHz}, \mathrm{CDCl}_{3}, \mathrm{rt}\right)$

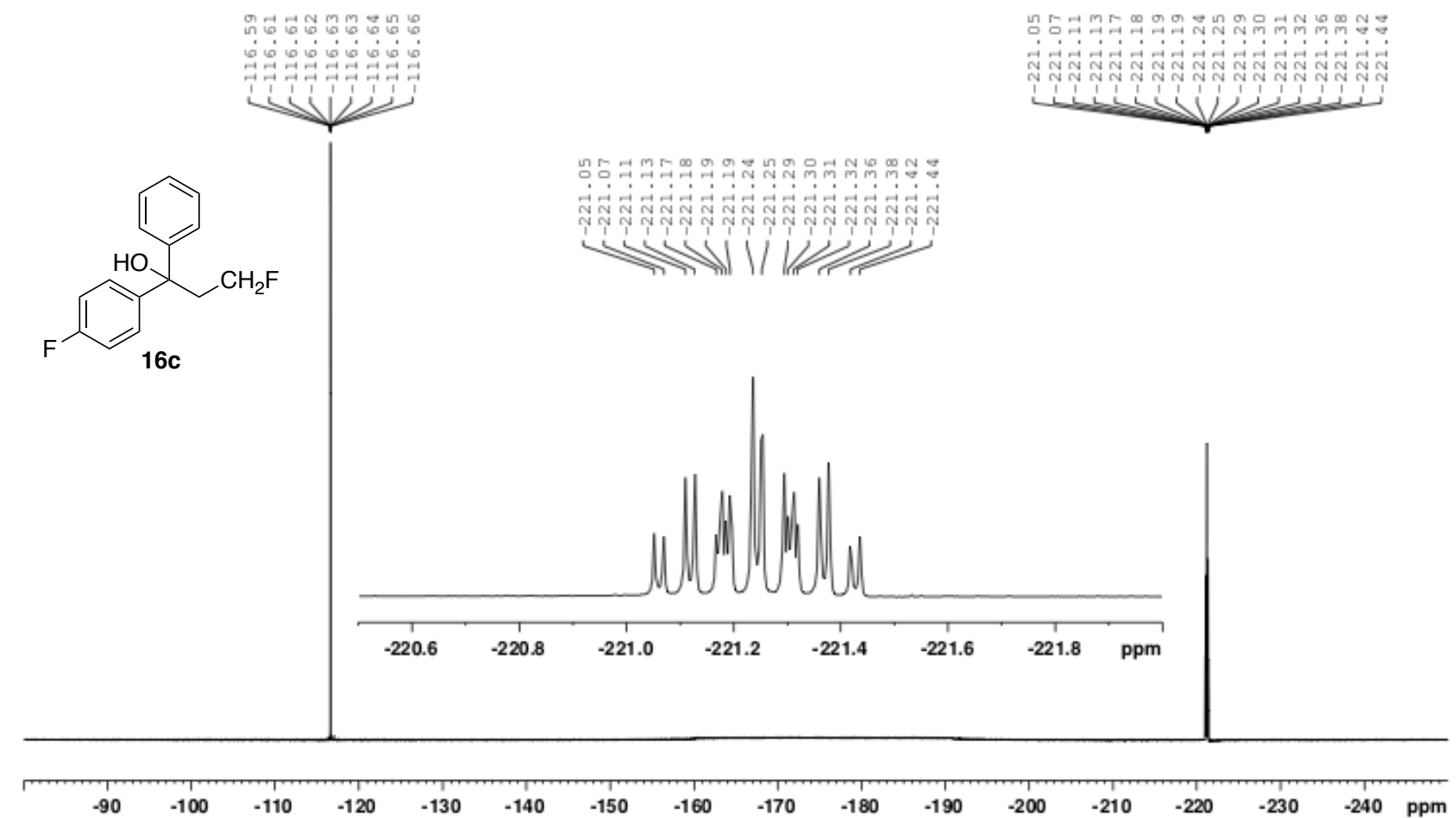

${ }^{1} \mathrm{H} \mathrm{NMR}\left(400 \mathrm{MHz}, \mathrm{CDCl}_{3}, \mathrm{rt}\right)$
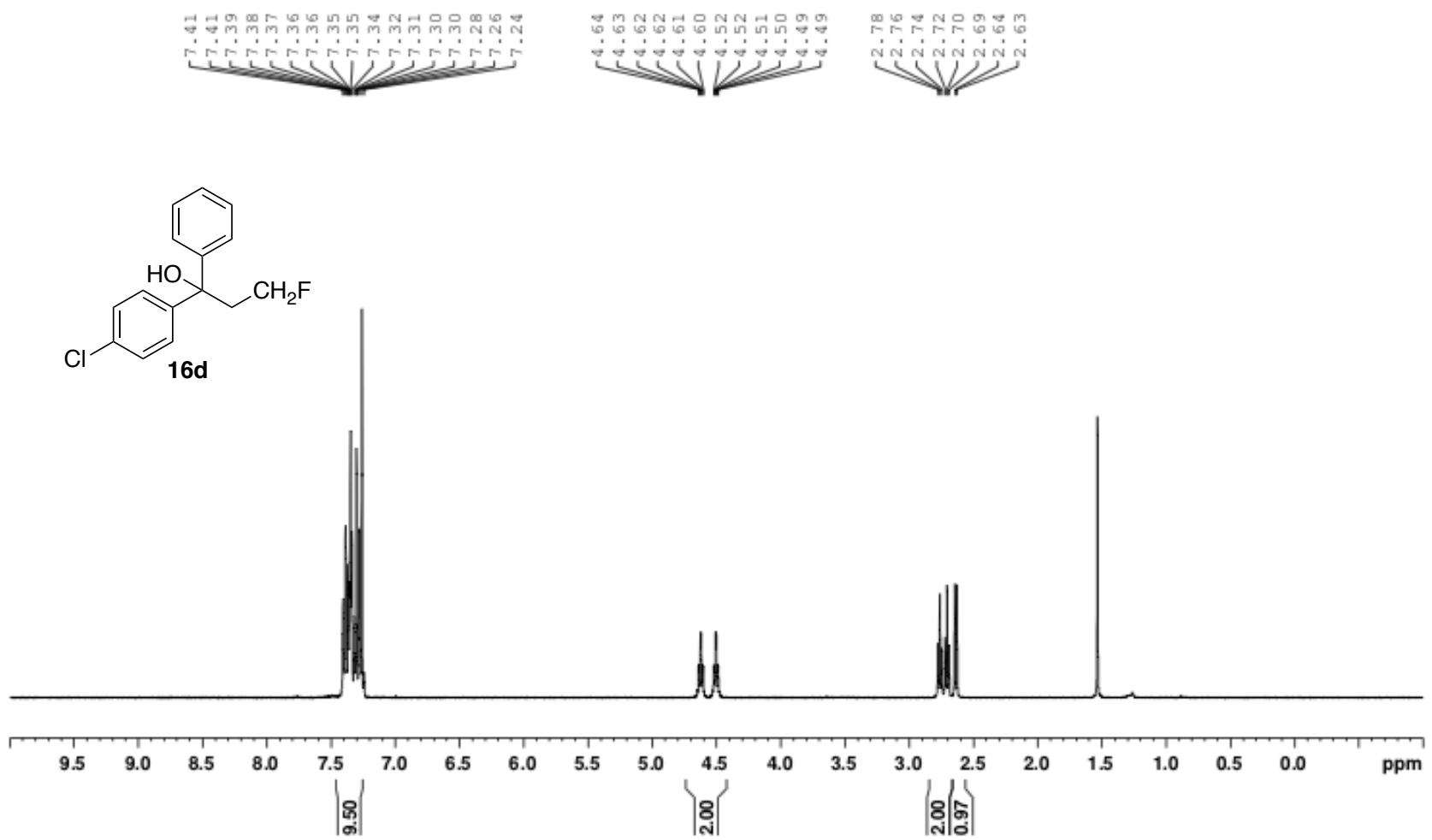
${ }^{13} \mathrm{C}$ NMR $\left(125 \mathrm{MHz}, \mathrm{CDCl}_{3}, \mathrm{rt}\right)$
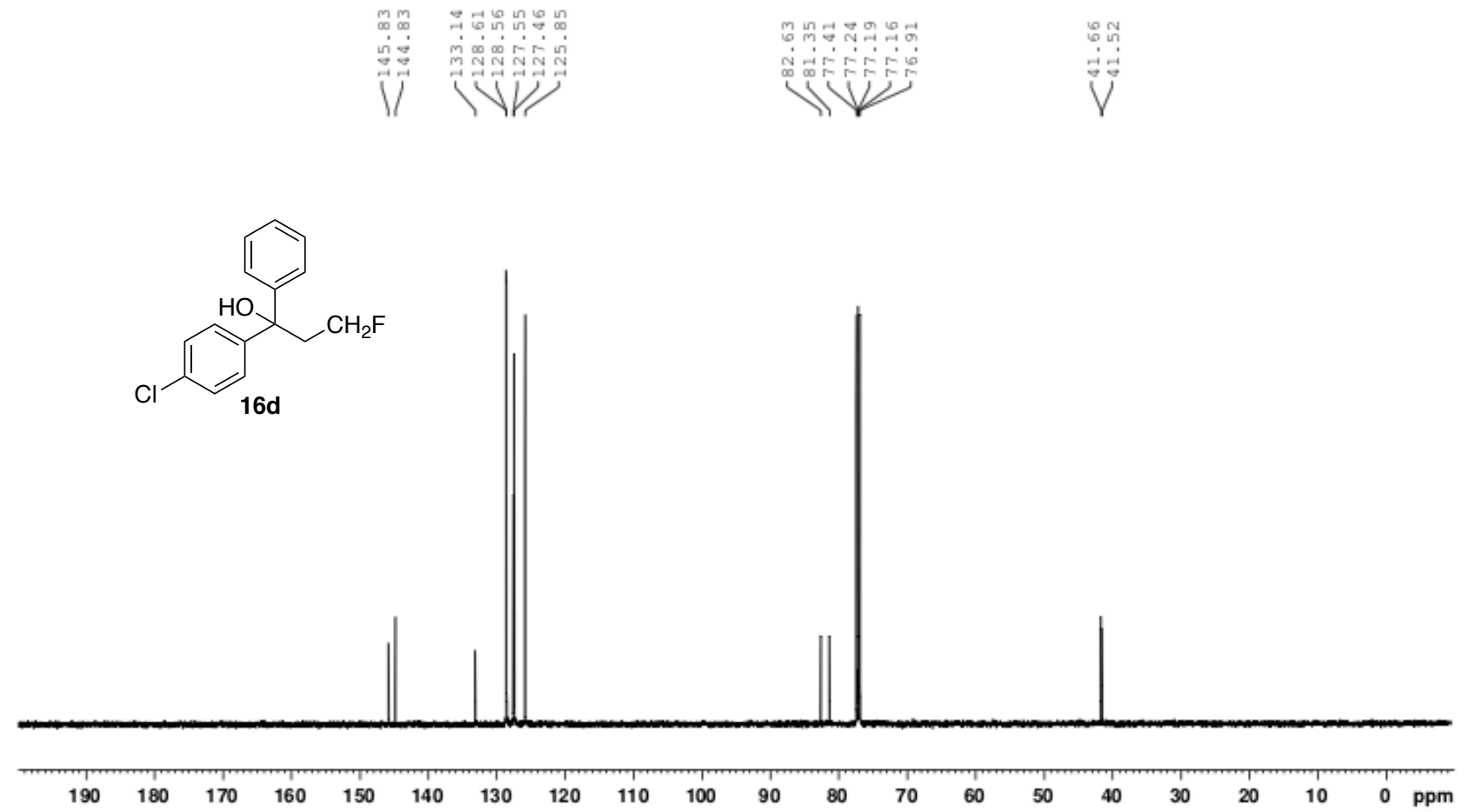

${ }^{19} \mathrm{~F} \mathrm{NMR}\left(376 \mathrm{MHz}, \mathrm{CDCl}_{3}, \mathrm{rt}\right)$

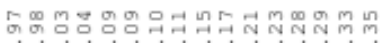

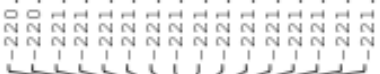

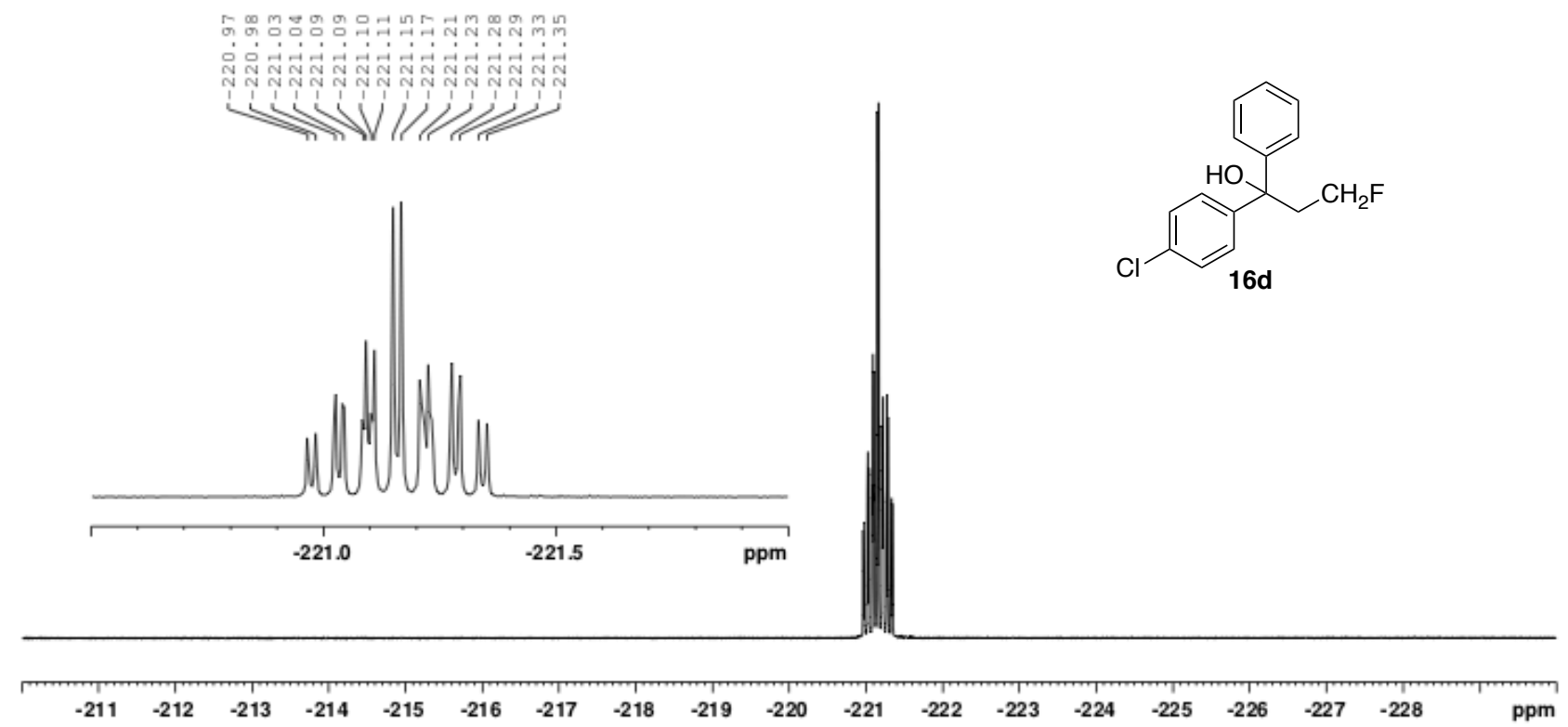


${ }^{1} \mathrm{H}$ NMR $\left(400 \mathrm{MHz}, \mathrm{CDCl}_{3}, \mathrm{rt}\right)$
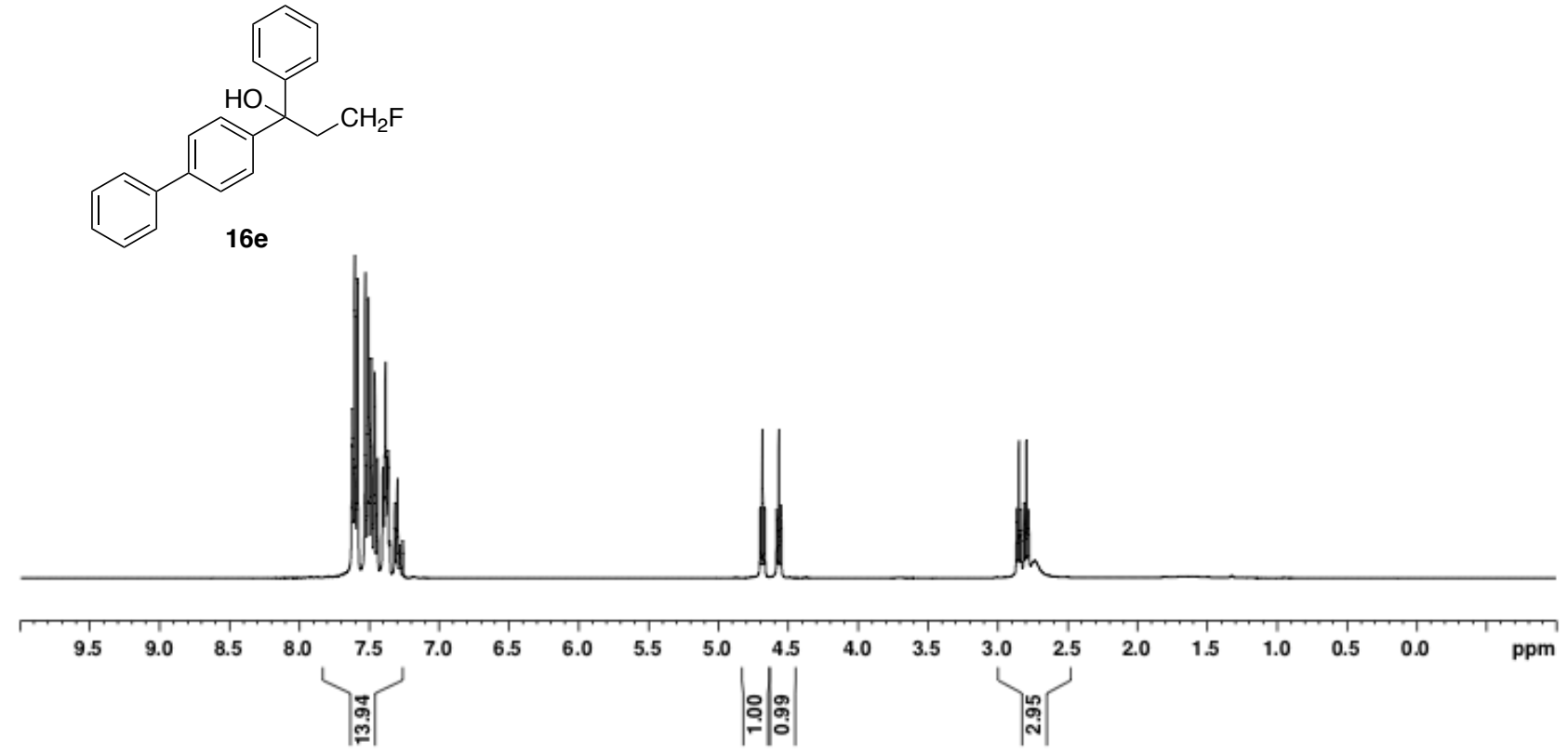

${ }^{13} \mathrm{C}$ NMR $\left(125 \mathrm{MHz}, \mathrm{CDCl}_{3}, \mathrm{rt}\right)$
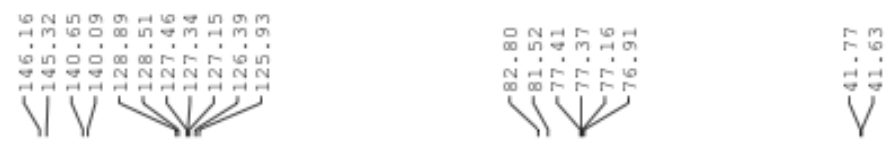<smiles>FCCC(Oc1ccccc1)(c1ccccc1)c1ccc(-c2ccccc2)cc1</smiles>

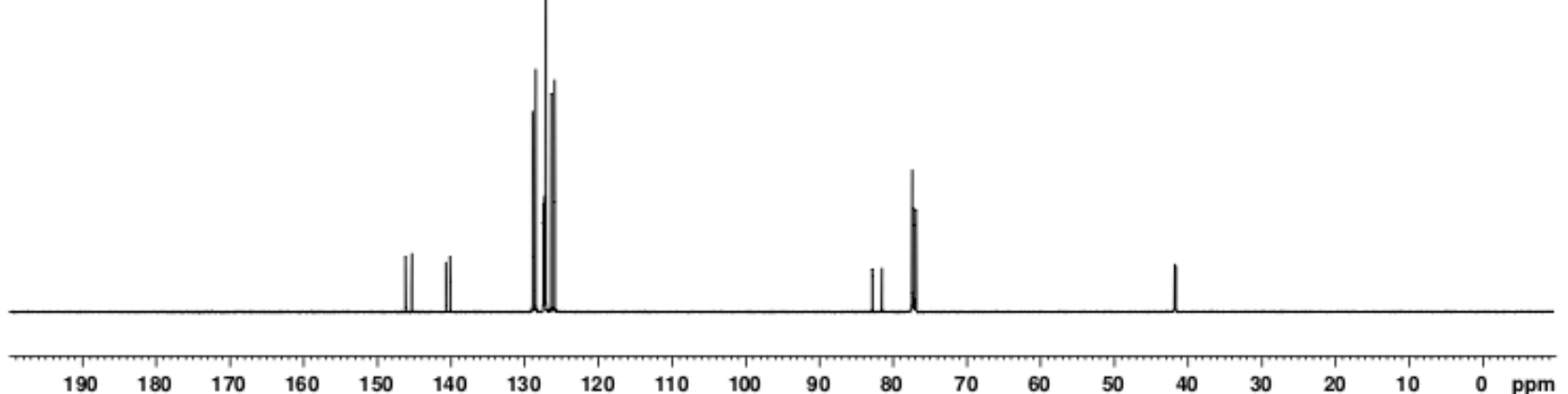


${ }^{19} \mathrm{~F} \mathrm{NMR}\left(376 \mathrm{MHz}, \mathrm{CDCl}_{3}, \mathrm{rt}\right)$

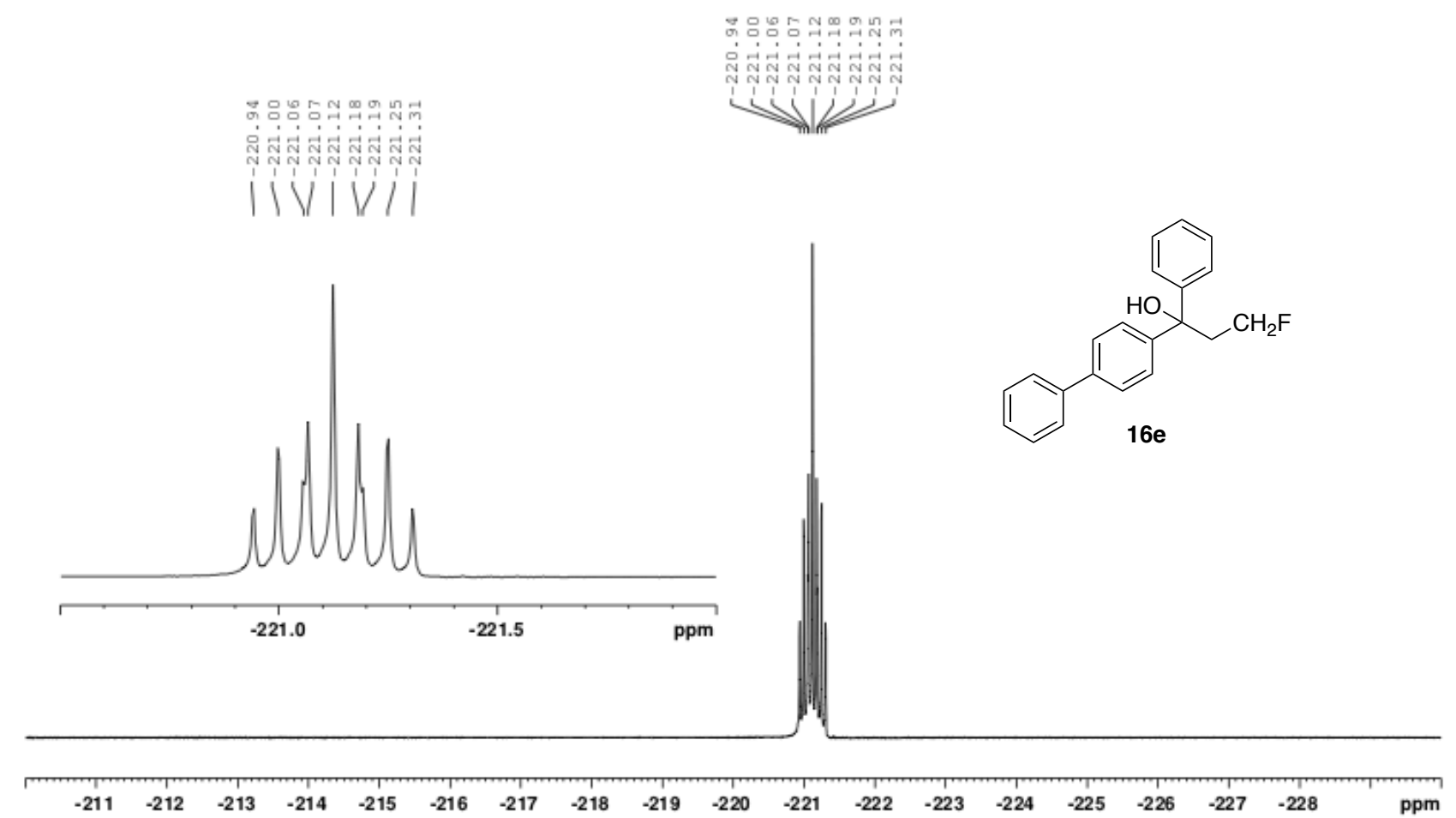

${ }^{1} \mathrm{H}$ NMR $\left(400 \mathrm{MHz}, \mathrm{CDCl}_{3}, \mathrm{rt}\right)$

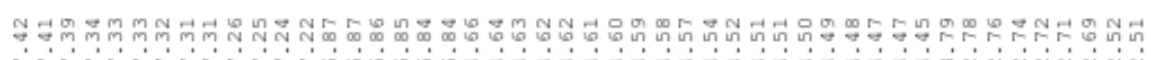

V V V<smiles>COc1ccc(C(O)(CCF)c2ccccc2)cc1</smiles>

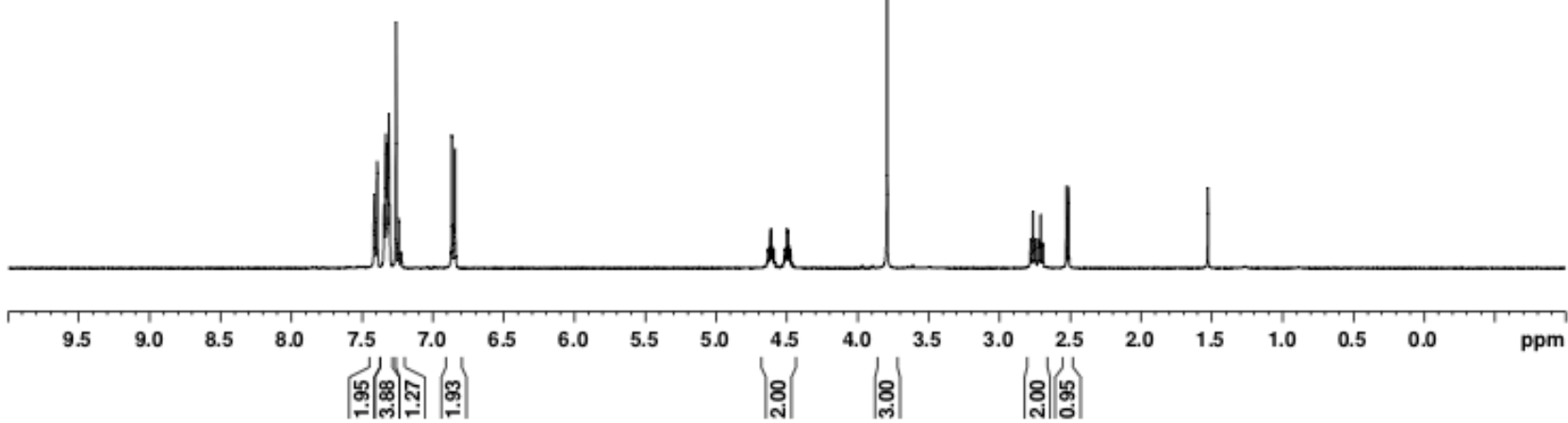


${ }^{13} \mathrm{C}$ NMR $\left(125 \mathrm{MHz}, \mathrm{CDCl}_{3}, \mathrm{rt}\right)$

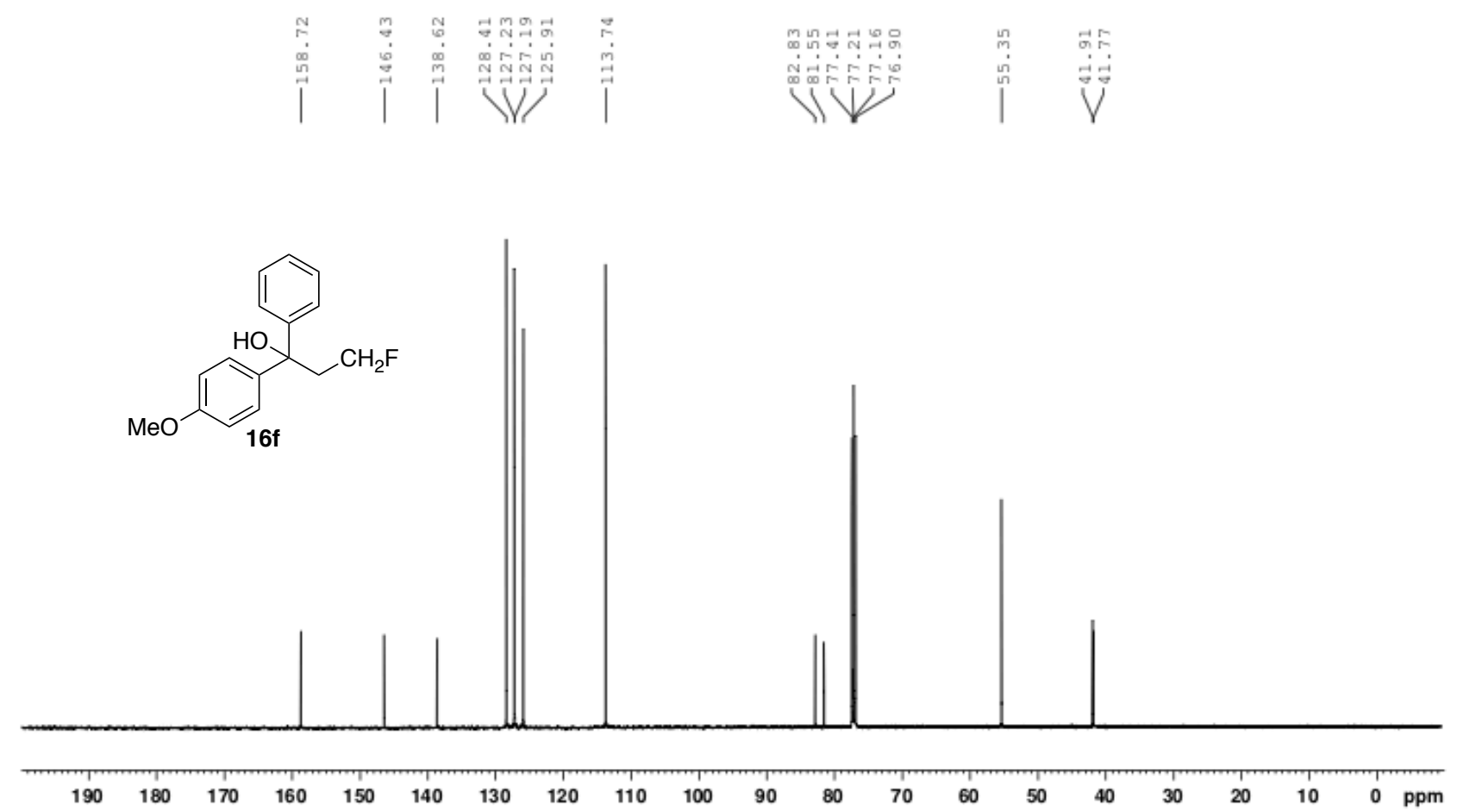

${ }^{19} \mathrm{~F}$ NMR $\left(376 \mathrm{MHz}, \mathrm{CDCl}_{3}, \mathrm{rt}\right)$

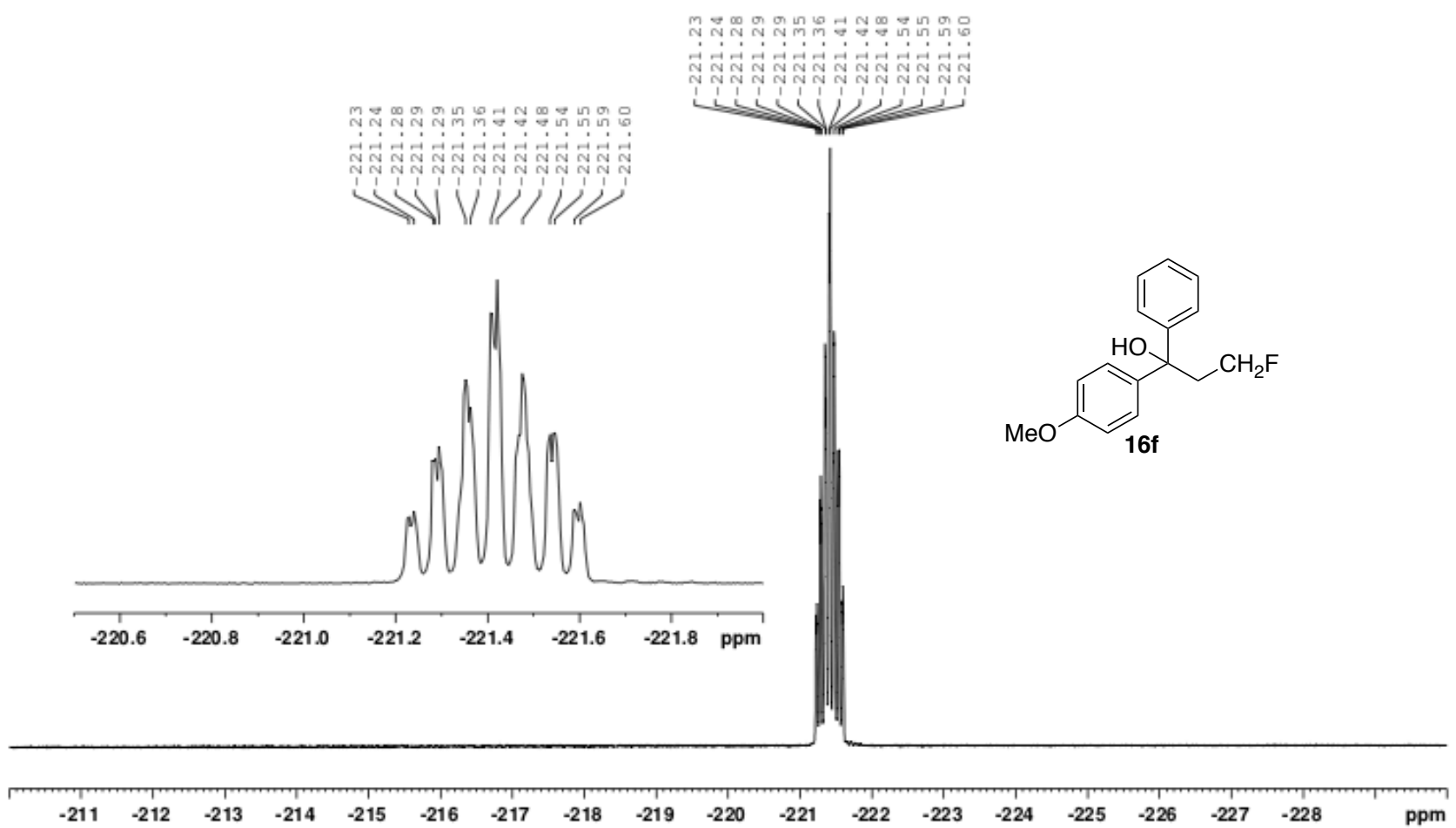


${ }^{1} \mathrm{H}$ NMR $\left(400 \mathrm{MHz}, \mathrm{CDCl}_{3}, \mathrm{rt}\right)$

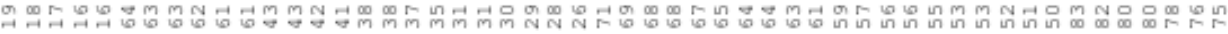

nom on

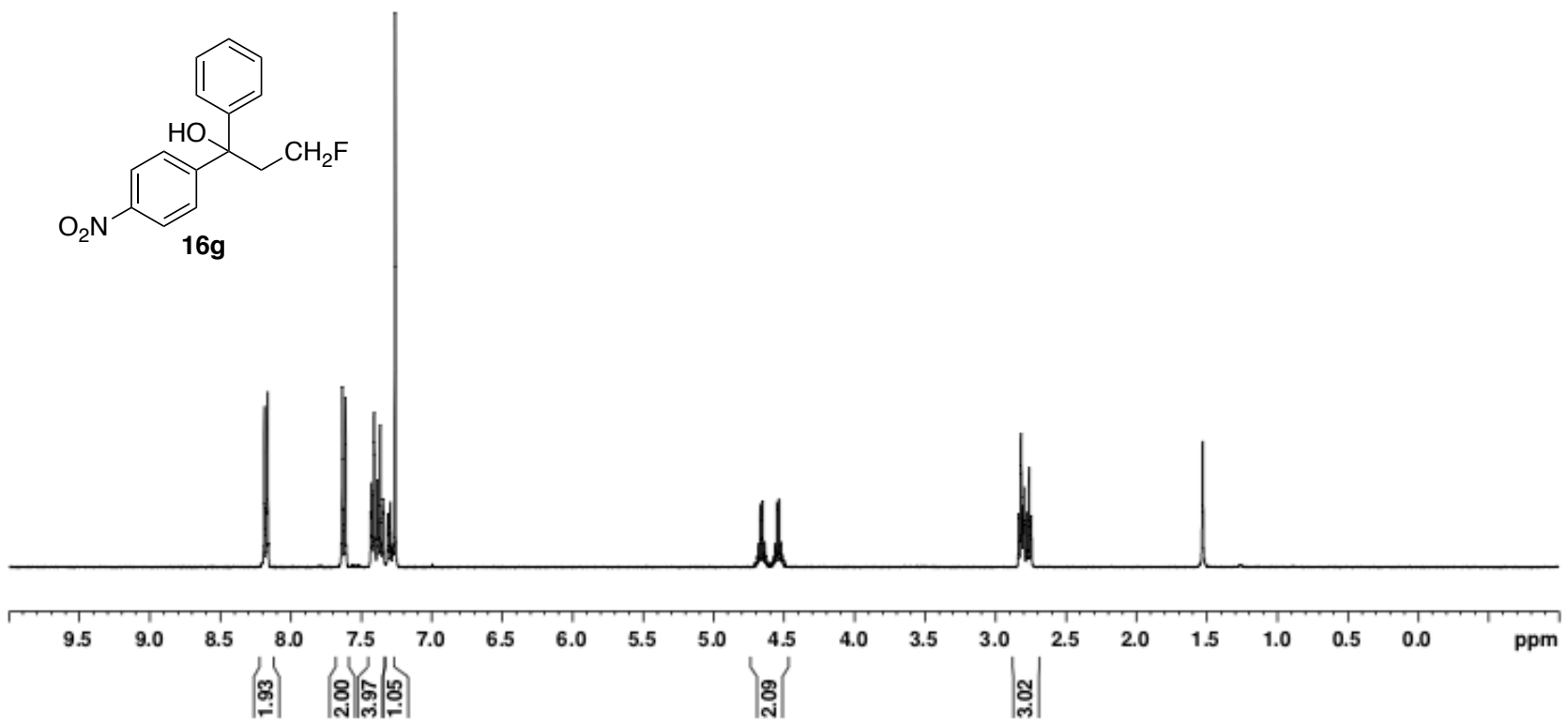

${ }^{13} \mathrm{C} \mathrm{NMR}\left(125 \mathrm{MHz}, \mathrm{CDCl}_{3}, \mathrm{rt}\right)$
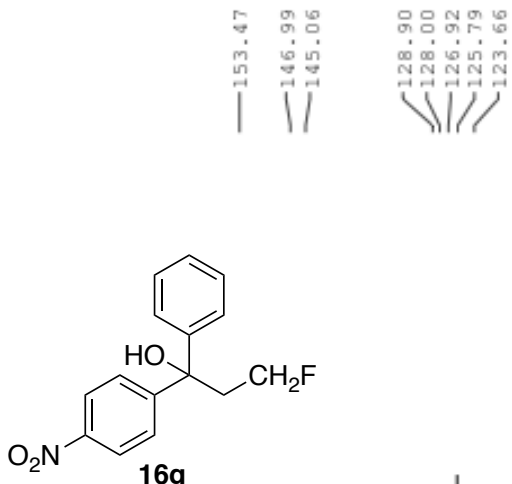

$16 \mathrm{~g}$

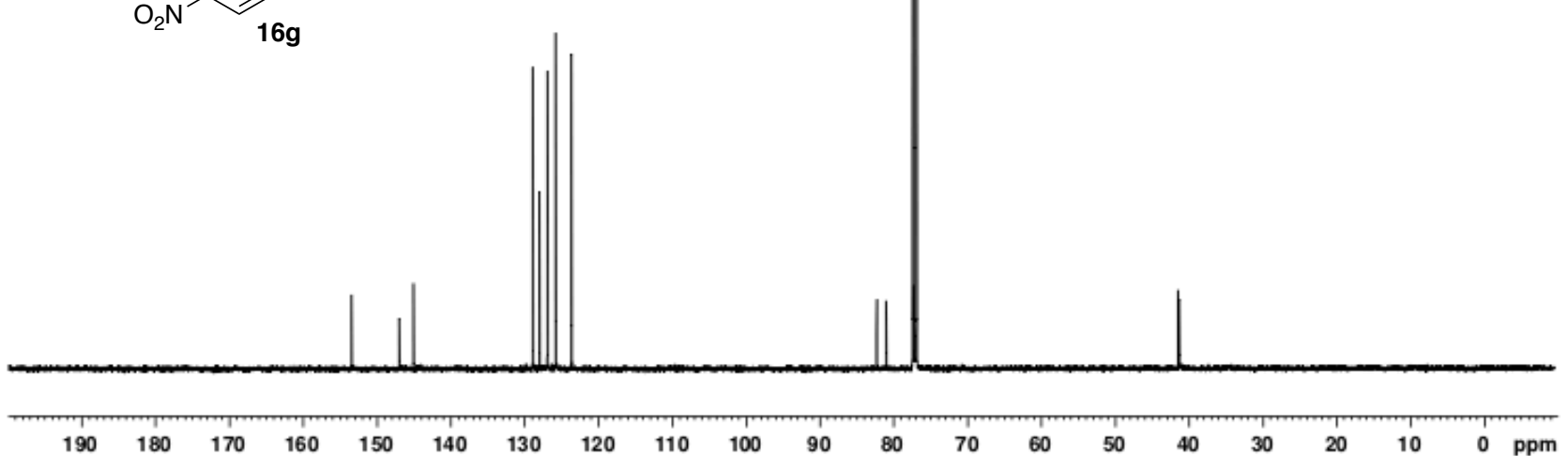


${ }^{19} \mathrm{~F} \mathrm{NMR}\left(376 \mathrm{MHz}, \mathrm{CDCl}_{3}, \mathrm{rt}\right)$

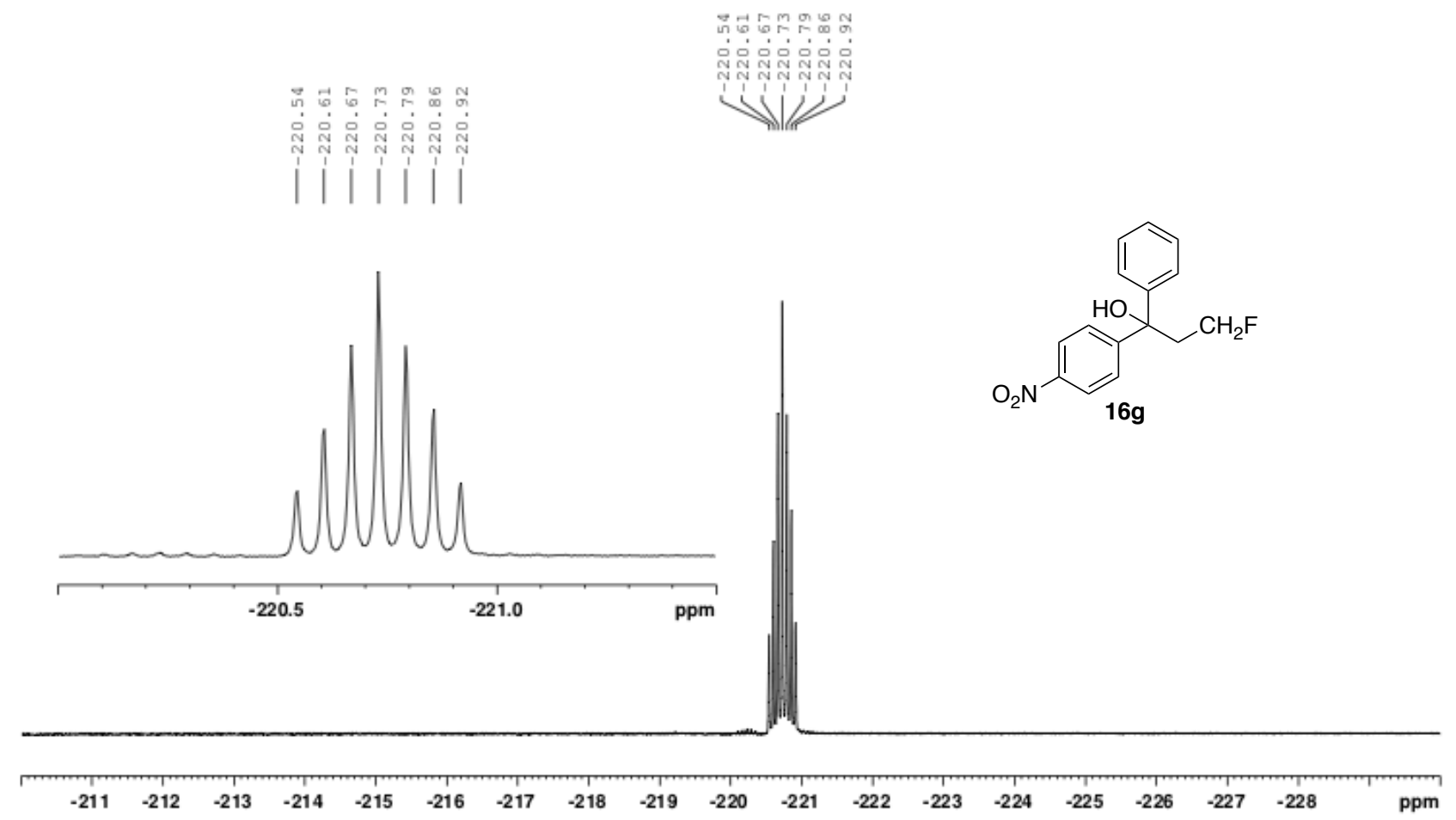

${ }^{1} \mathrm{H} \mathrm{NMR}\left(400 \mathrm{MHz}, \mathrm{CDCl}_{3}, \mathrm{rt}\right)$
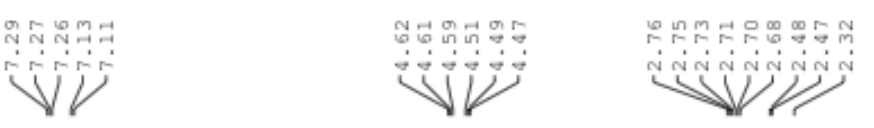<smiles>Cc1ccc(C(O)(CCF)c2ccc([N+](=O)[O-])cc2)cc1</smiles>

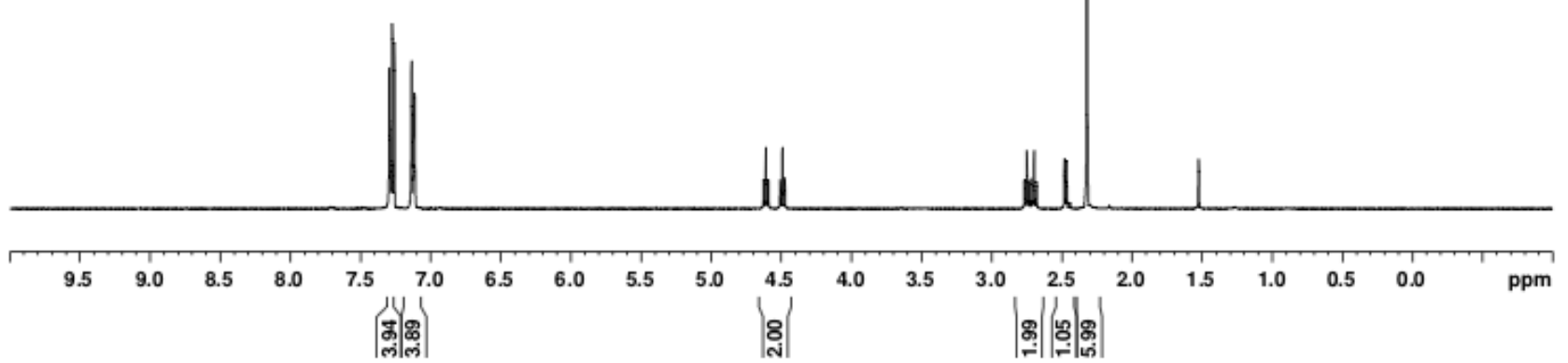


${ }^{13} \mathrm{C}$ NMR $\left(125 \mathrm{MHz}, \mathrm{CDCl}_{3}, \mathrm{rt}\right)$
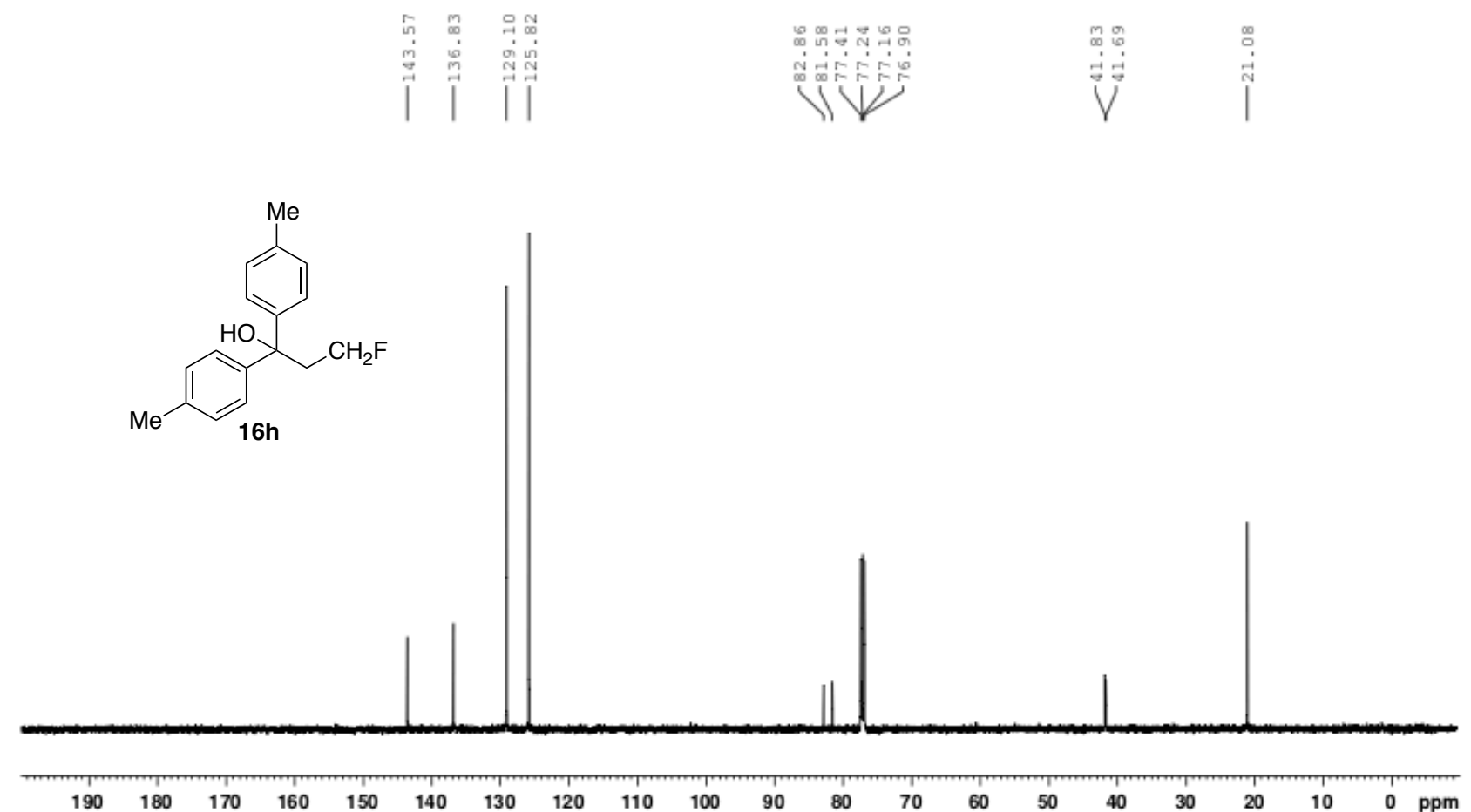

${ }^{19} \mathrm{~F} \mathrm{NMR}\left(376 \mathrm{MHz}, \mathrm{CDCl}_{3}, \mathrm{rt}\right)$

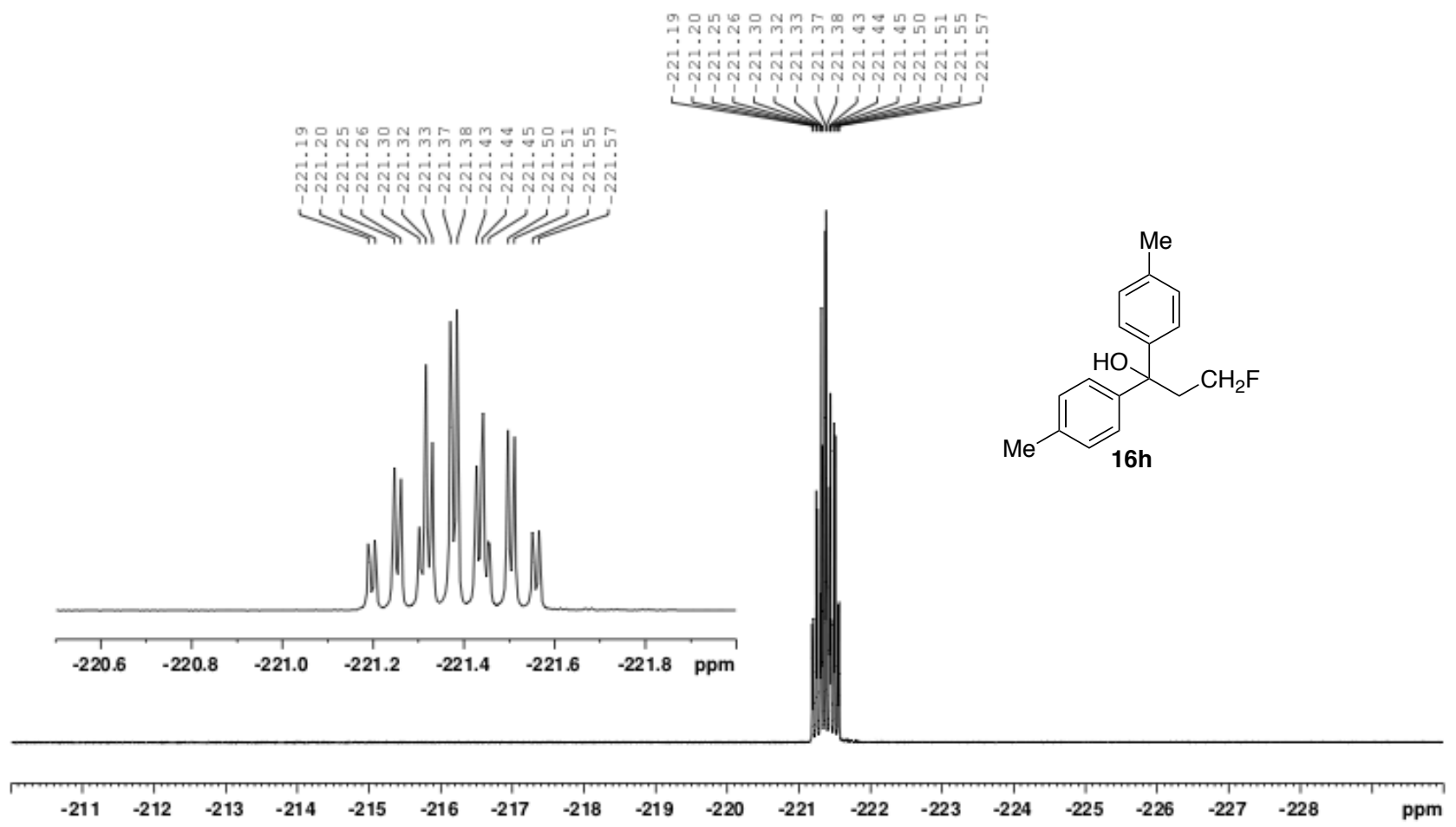


${ }^{1} \mathrm{H}$ NMR $\left(400 \mathrm{MHz}, \mathrm{CDCl}_{3}, \mathrm{rt}\right)$

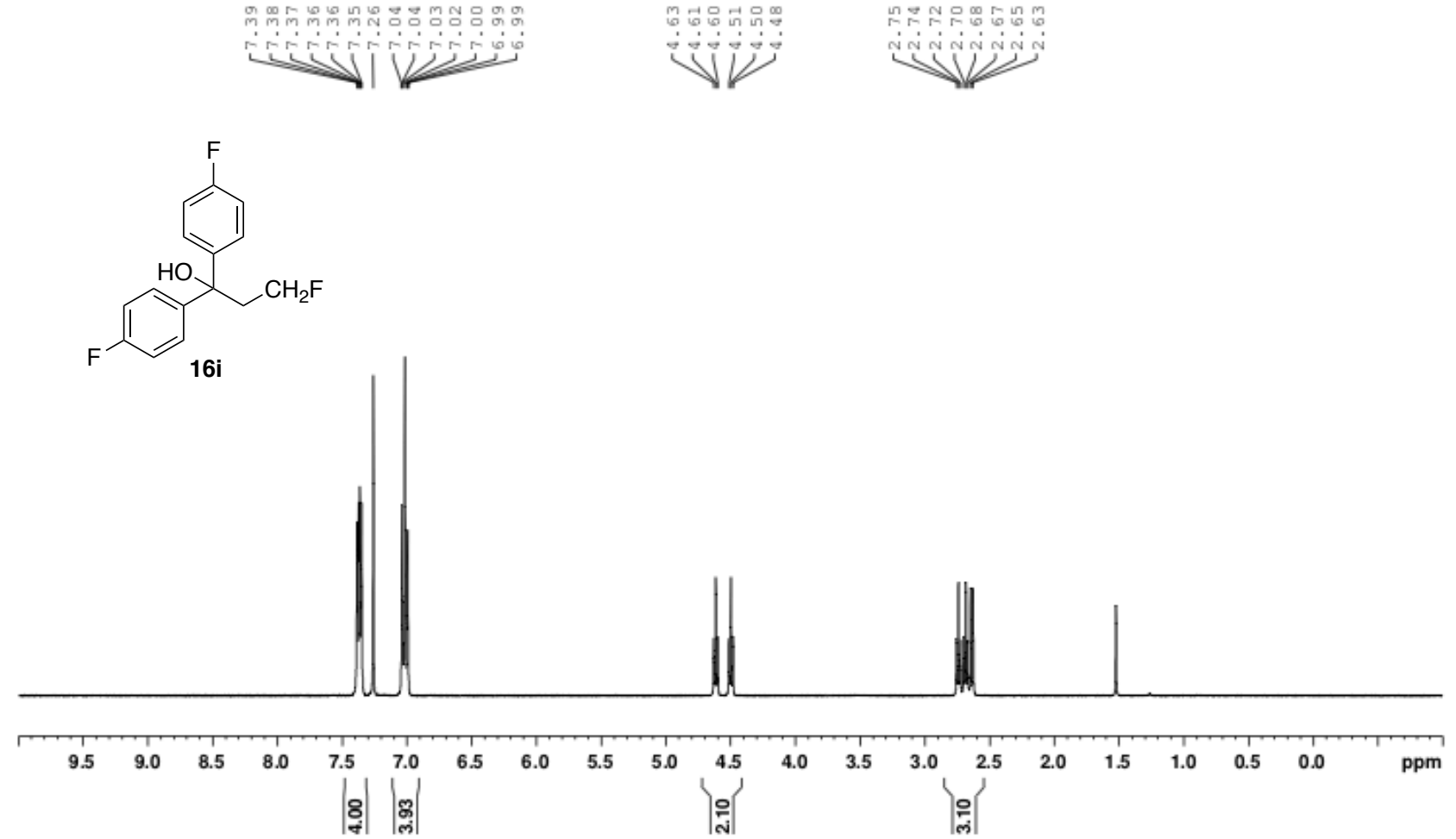

${ }^{13} \mathrm{C}$ NMR (125 MHz, $\left.\mathrm{CDCl}_{3}, \mathrm{rt}\right)$
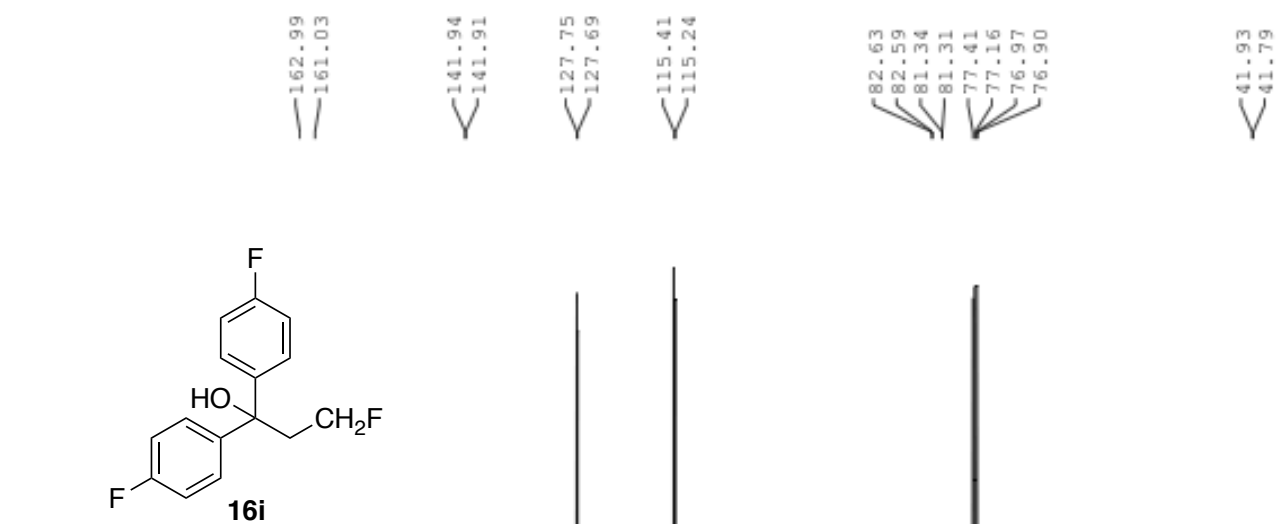

$190 \quad 180 \quad 170$
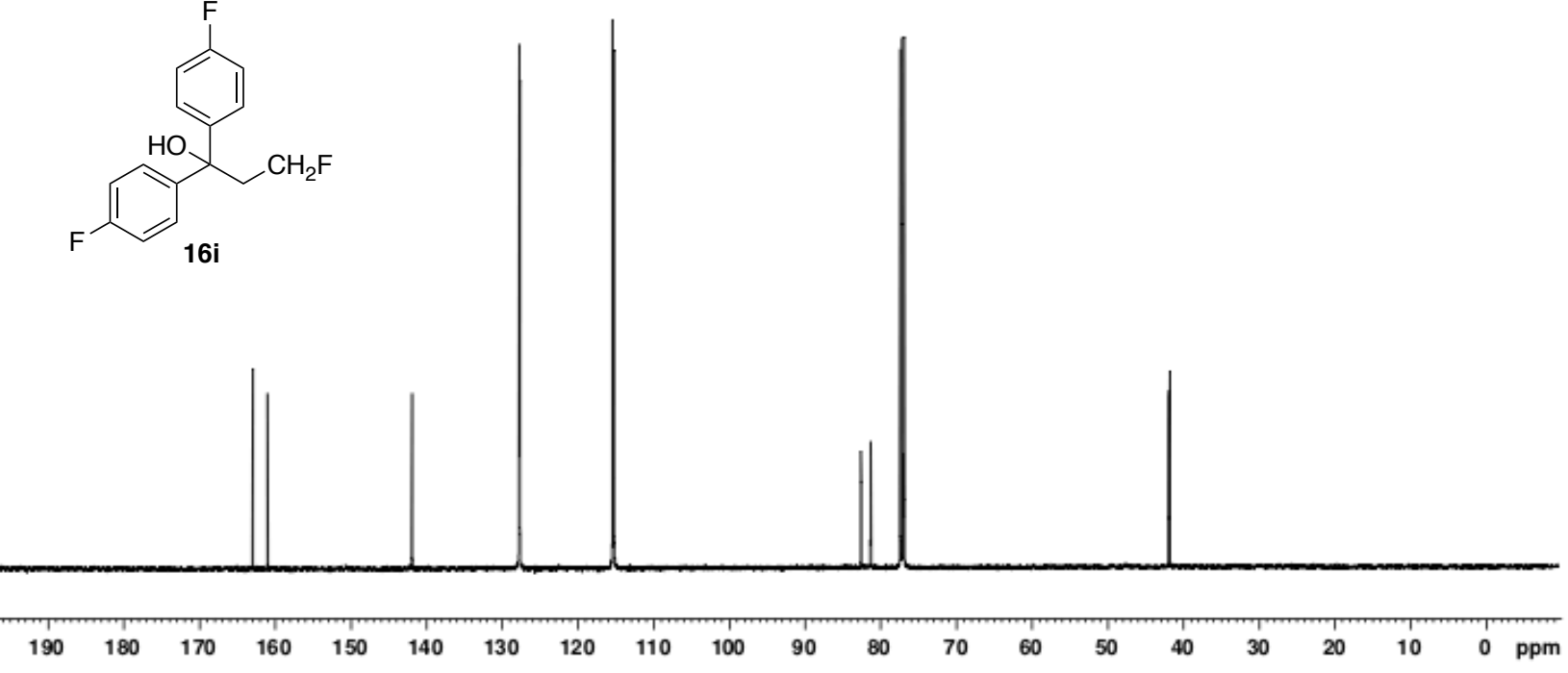
${ }^{19} \mathrm{~F} \mathrm{NMR}\left(376 \mathrm{MHz}, \mathrm{CDCl}_{3}, \mathrm{rt}\right)$

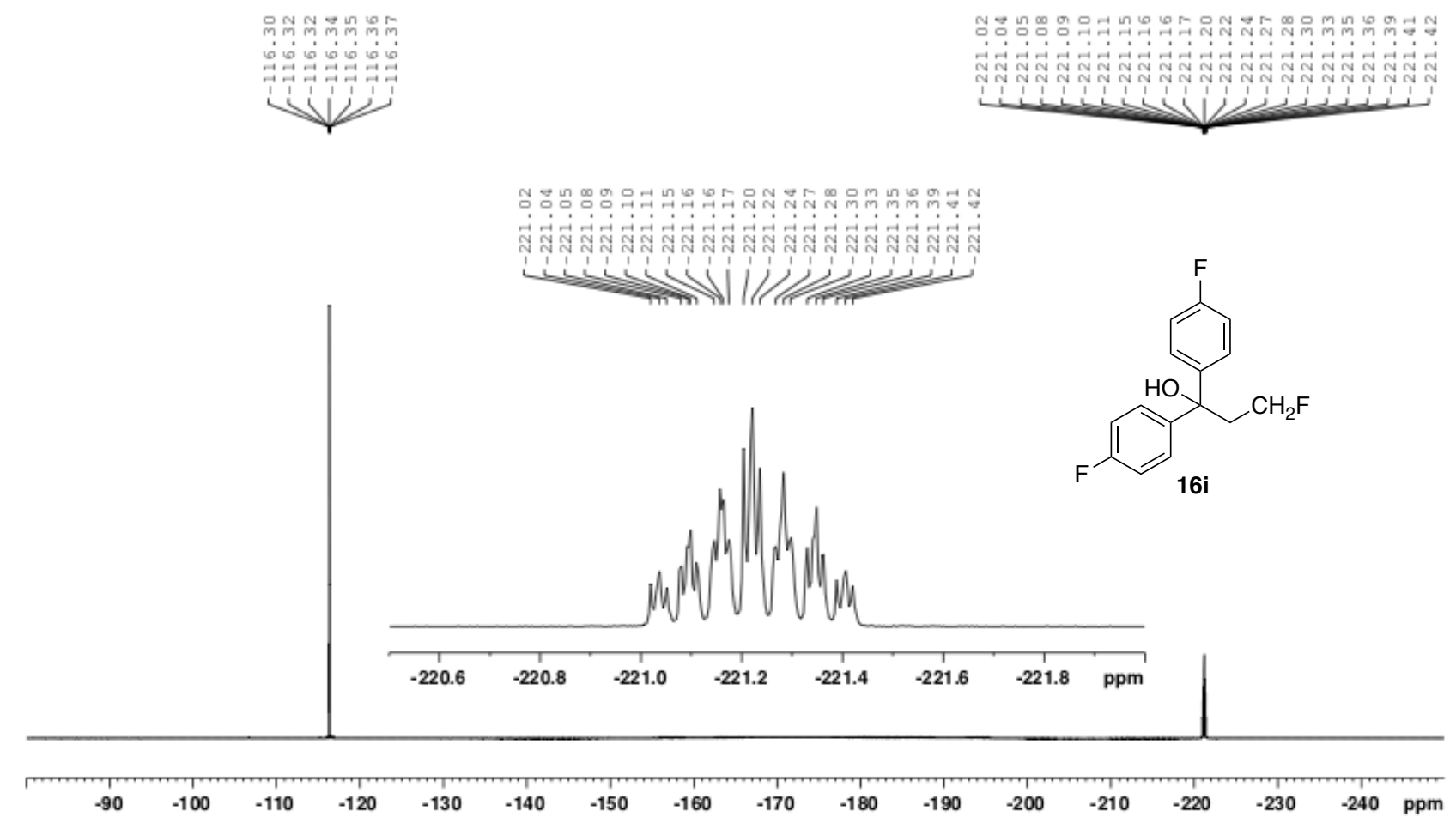

${ }^{1} \mathrm{H}$ NMR $\left(400 \mathrm{MHz}, \mathrm{CDCl}_{3}, \mathrm{rt}\right)$
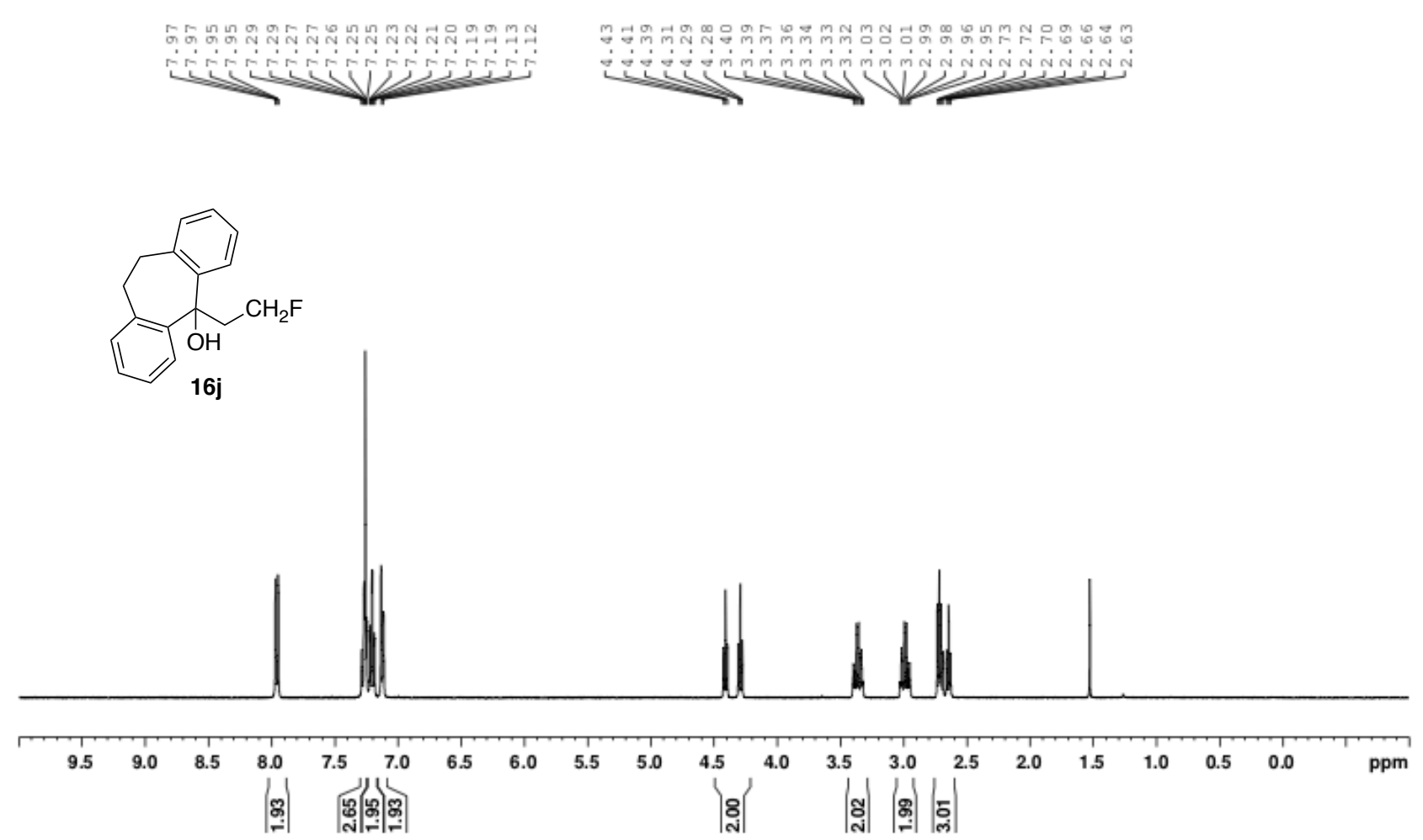
${ }^{13} \mathrm{C}$ NMR $\left(125 \mathrm{MHz}, \mathrm{CDCl}_{3}, \mathrm{rt}\right)$
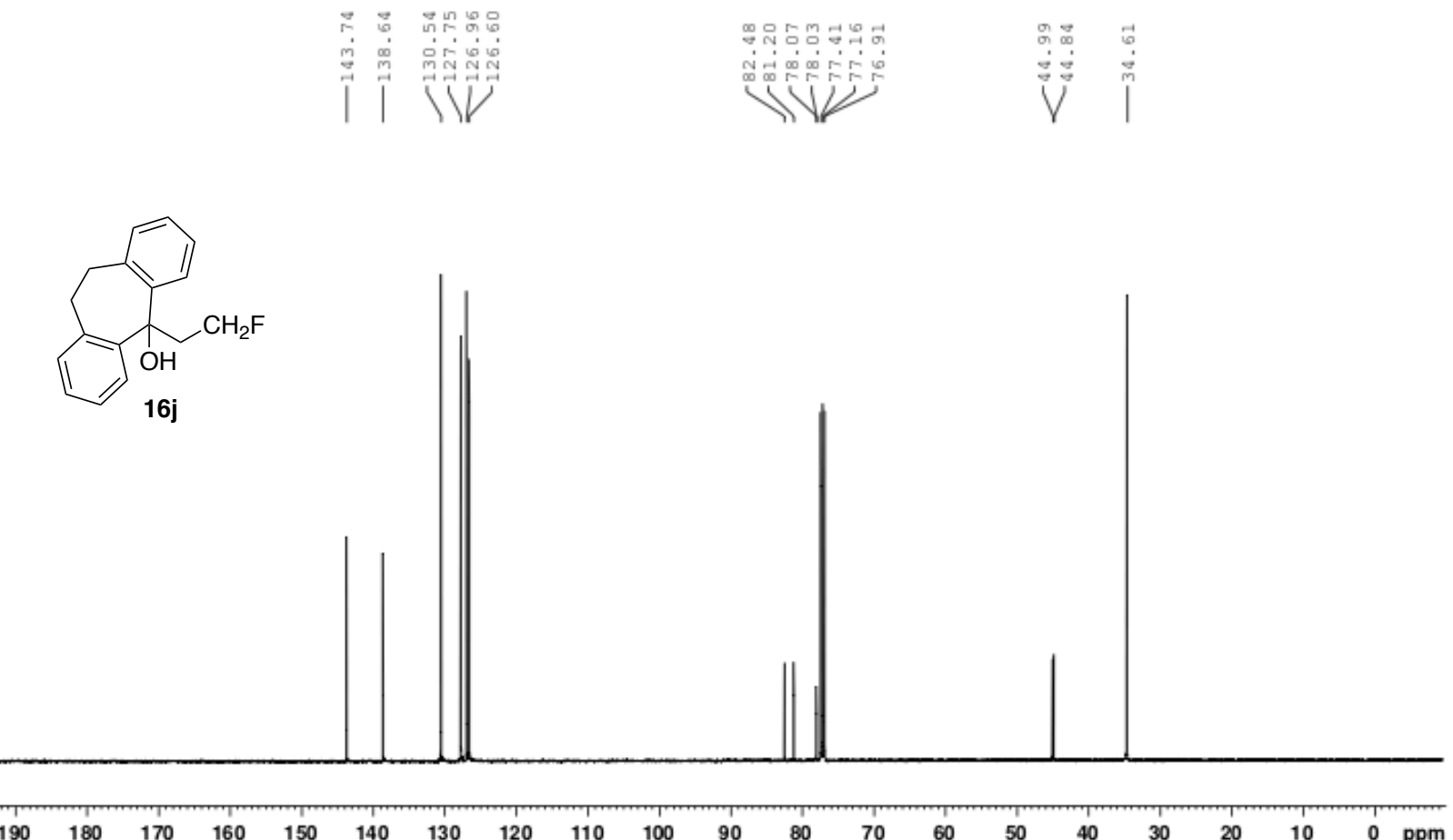

${ }^{19} \mathrm{~F} \mathrm{NMR}\left(376 \mathrm{MHz}, \mathrm{CDCl}_{3}, \mathrm{rt}\right)$

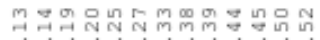

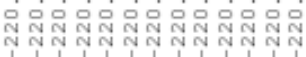

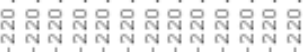

viviviviti
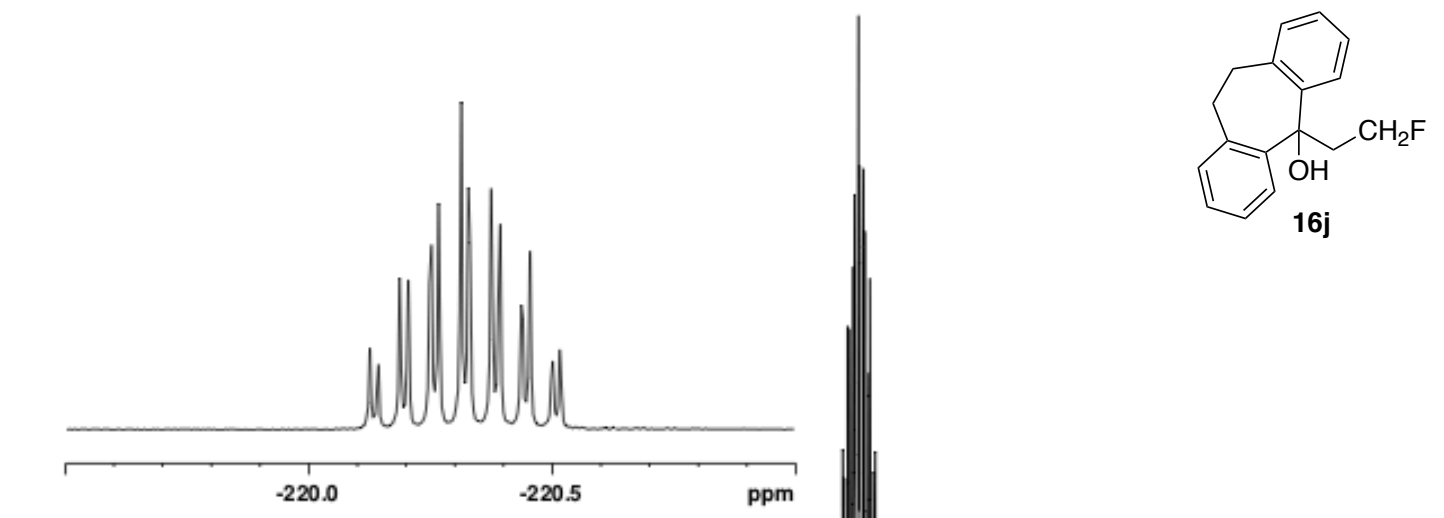

$\mathrm{pm}$

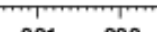

$-223-2$ 
${ }^{1} \mathrm{H}$ NMR $\left(400 \mathrm{MHz}, \mathrm{CDCl}_{3}, \mathrm{rt}\right)$

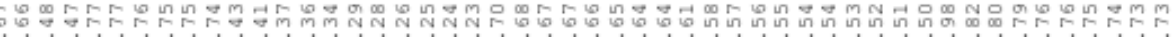

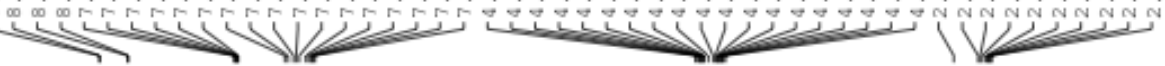

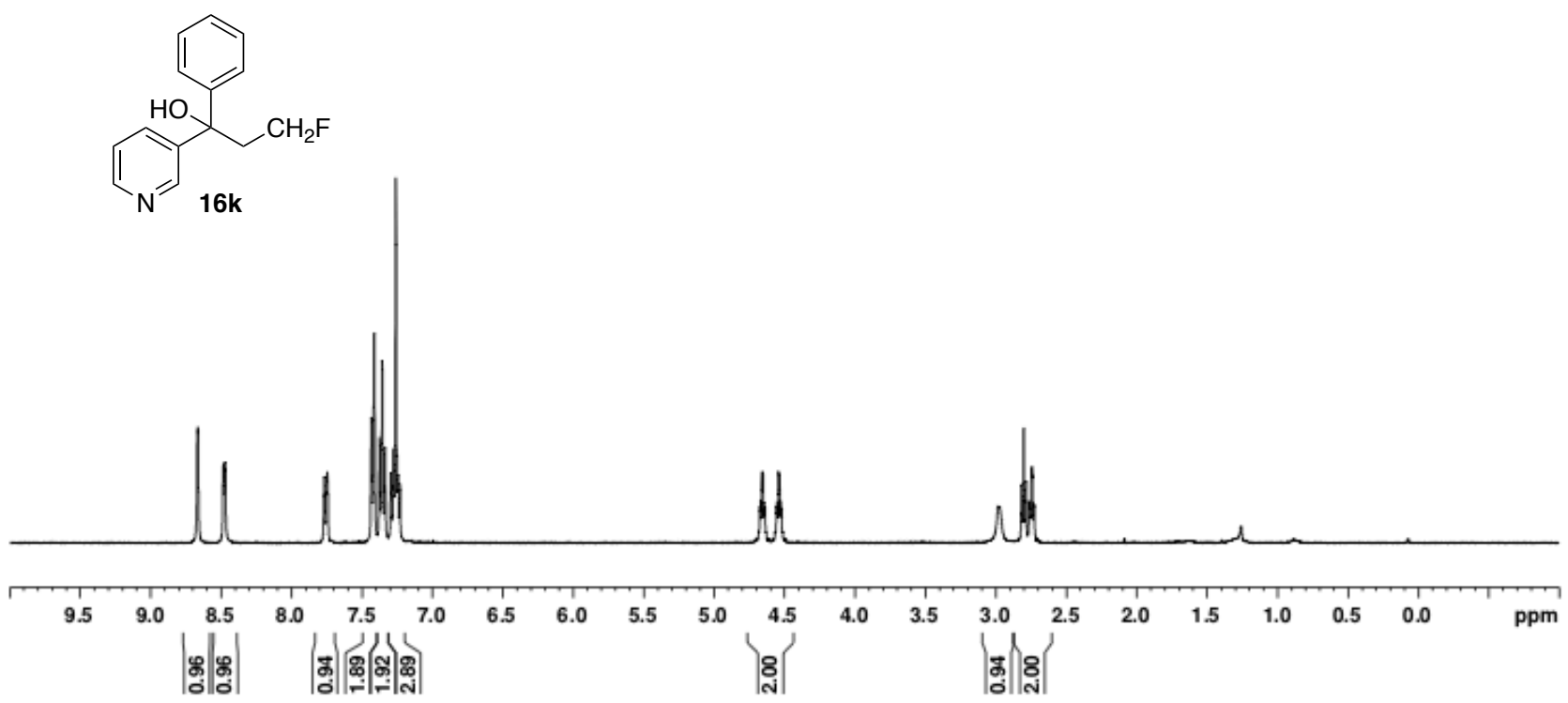

${ }^{13} \mathrm{C}$ NMR $\left(125 \mathrm{MHz}, \mathrm{CDCl}_{3}, \mathrm{rt}\right)$
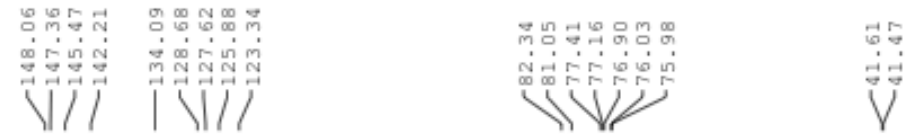<smiles>O=C(c1ccccc1)C(CCF)(c1ccccn1)c1ccccn1</smiles>

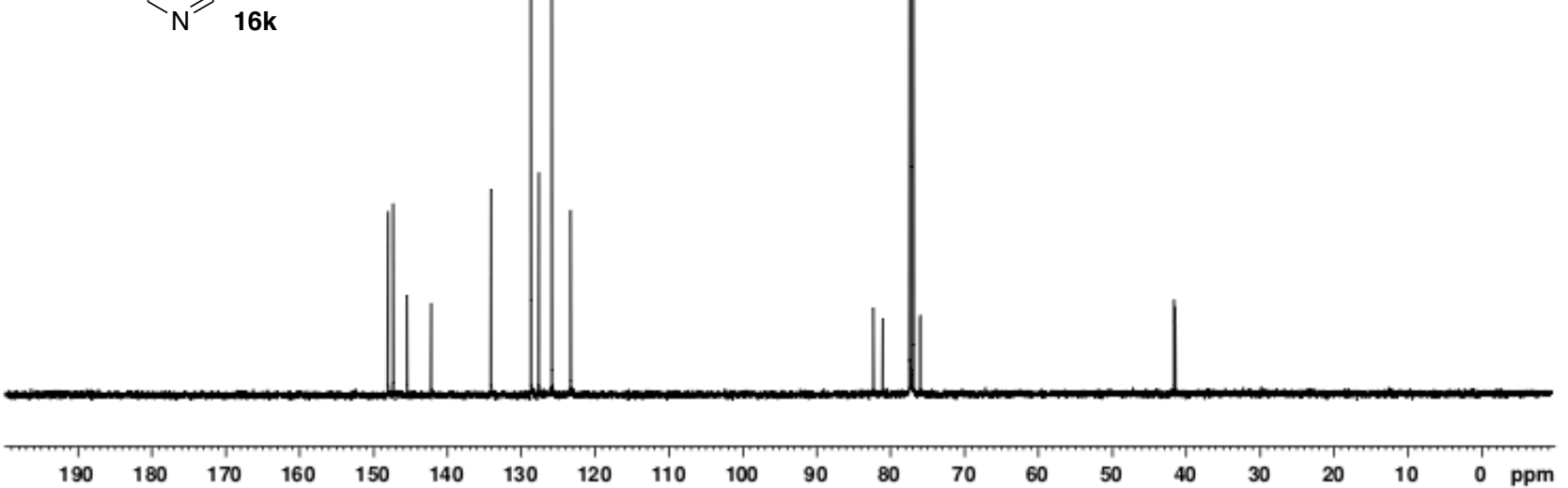


${ }^{19} \mathrm{~F} \mathrm{NMR}\left(376 \mathrm{MHz}, \mathrm{CDCl}_{3}, \mathrm{rt}\right)$

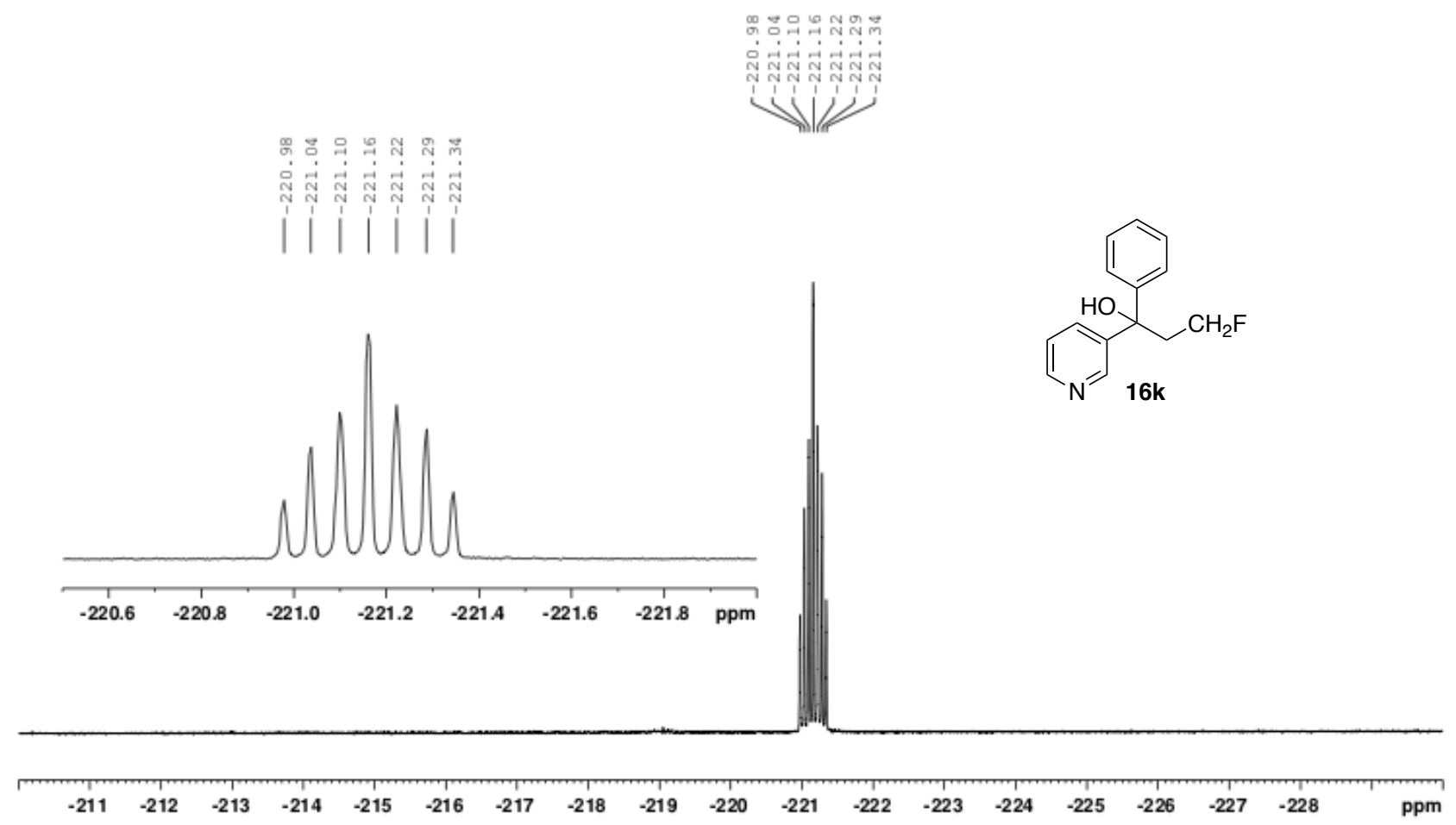

${ }^{1} \mathrm{H} \mathrm{NMR} \mathrm{(400} \mathrm{MHz,} \mathrm{CDCl}_{3}$, rt)

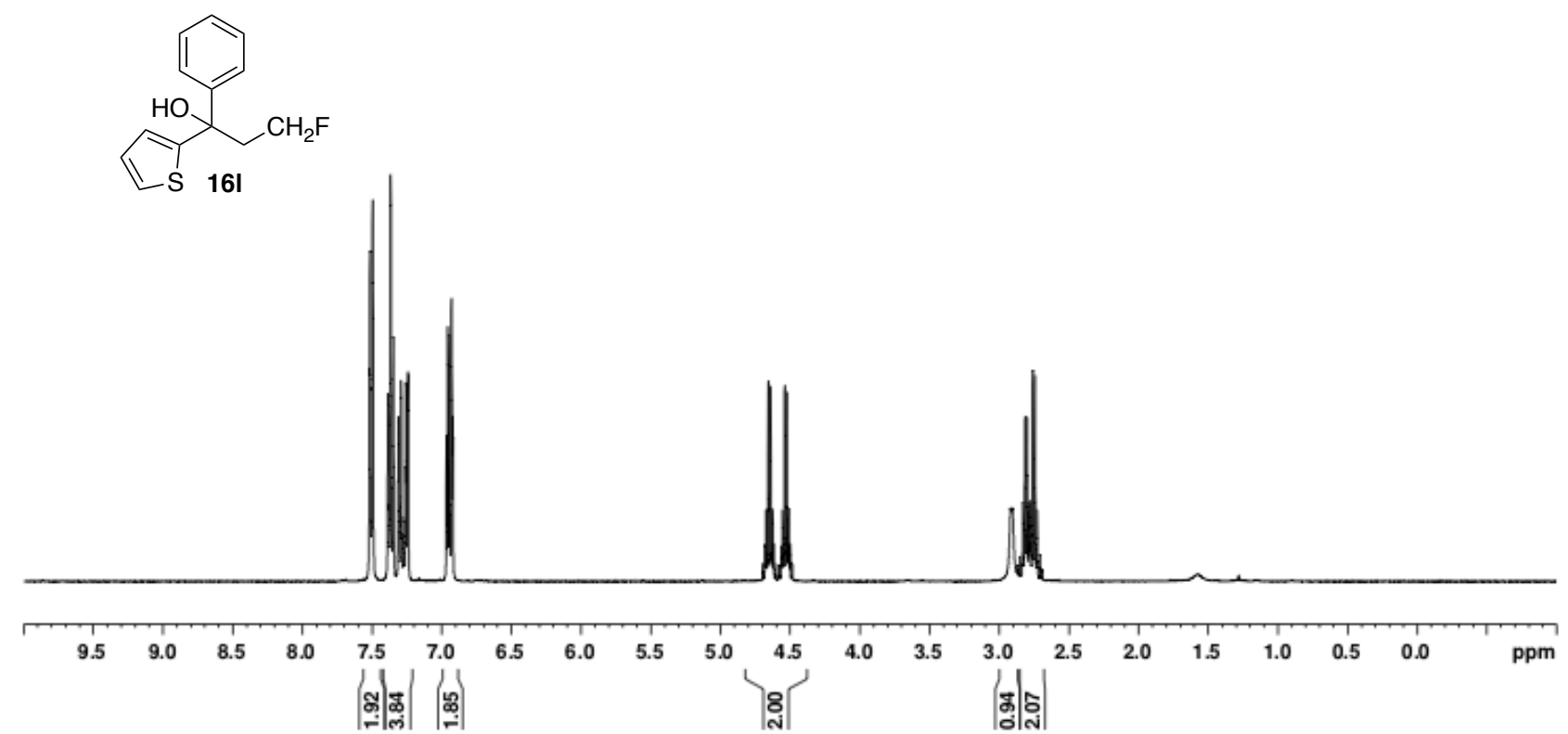


${ }^{13} \mathrm{C}$ NMR (125 MHz, $\left.\mathrm{CDCl}_{3}, \mathrm{rt}\right)$

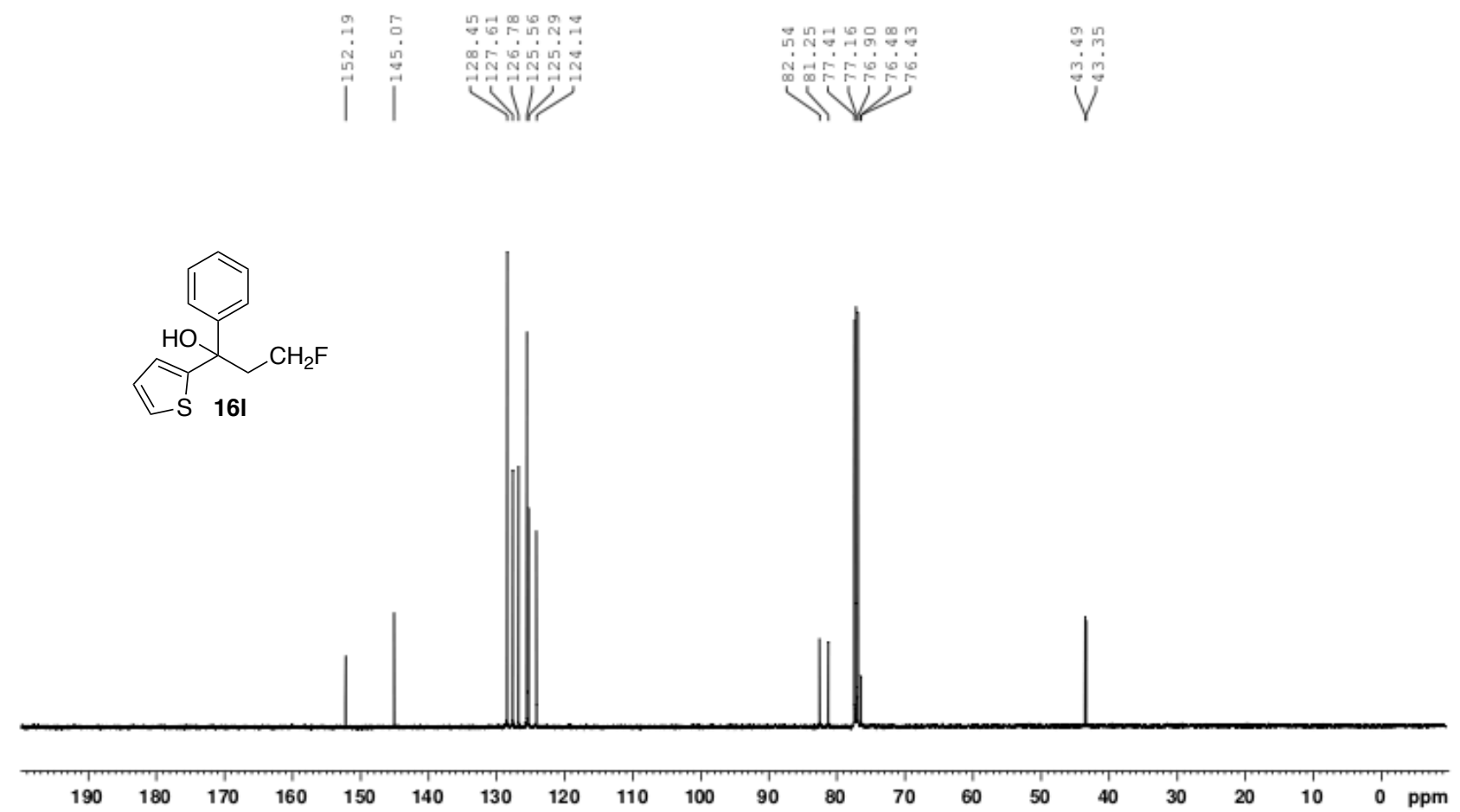

${ }^{19}$ F NMR (376 MHz, $\left.\mathrm{CDCl}_{3}, \mathrm{rt}\right)$

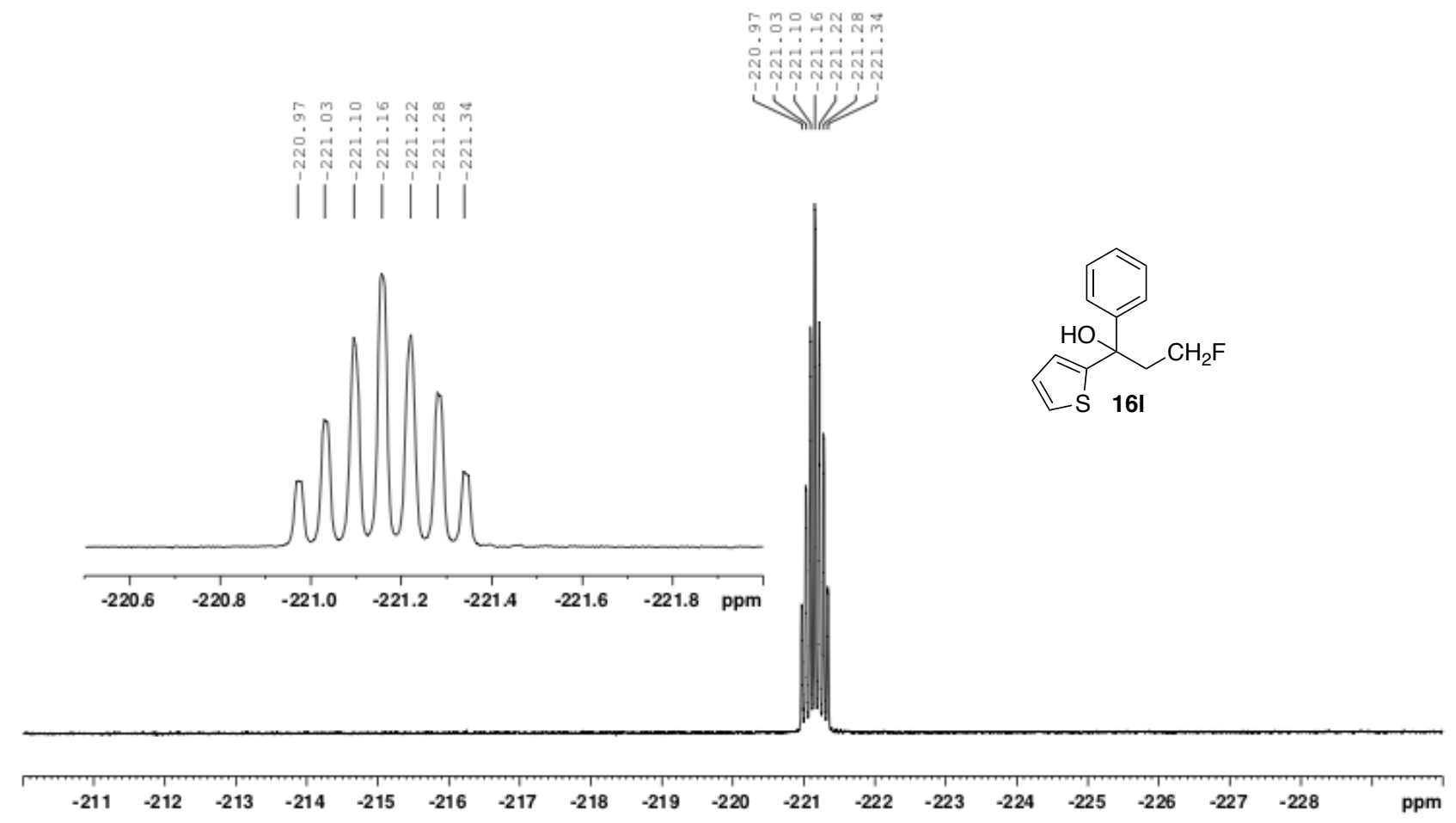


${ }^{1} \mathrm{H}$ NMR $\left(400 \mathrm{MHz}, \mathrm{CDCl}_{3}, \mathrm{rt}\right)$

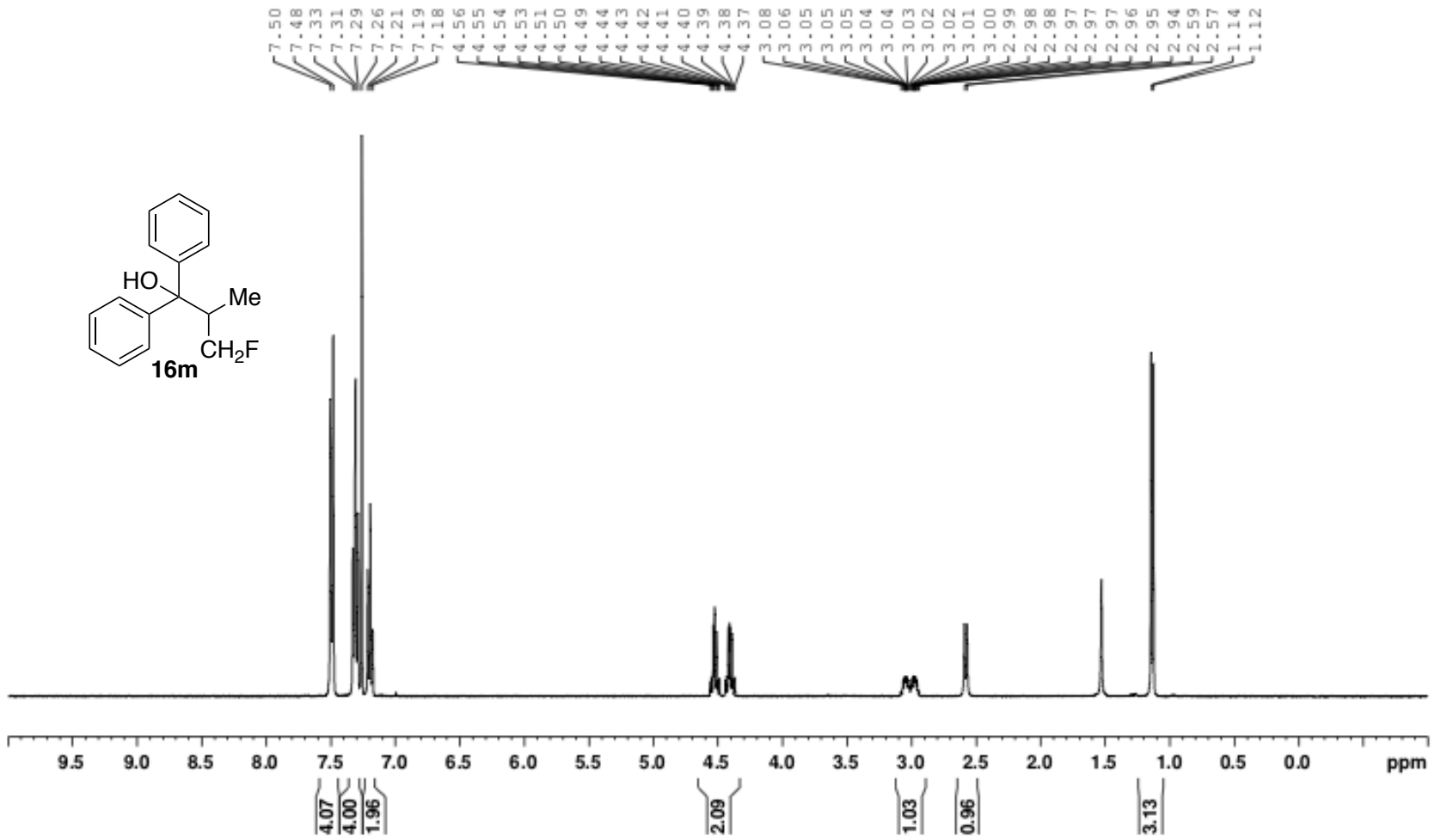

${ }^{13} \mathrm{C}$ NMR $\left(125 \mathrm{MHz}, \mathrm{CDCl}_{3}, \mathrm{rt}\right)$
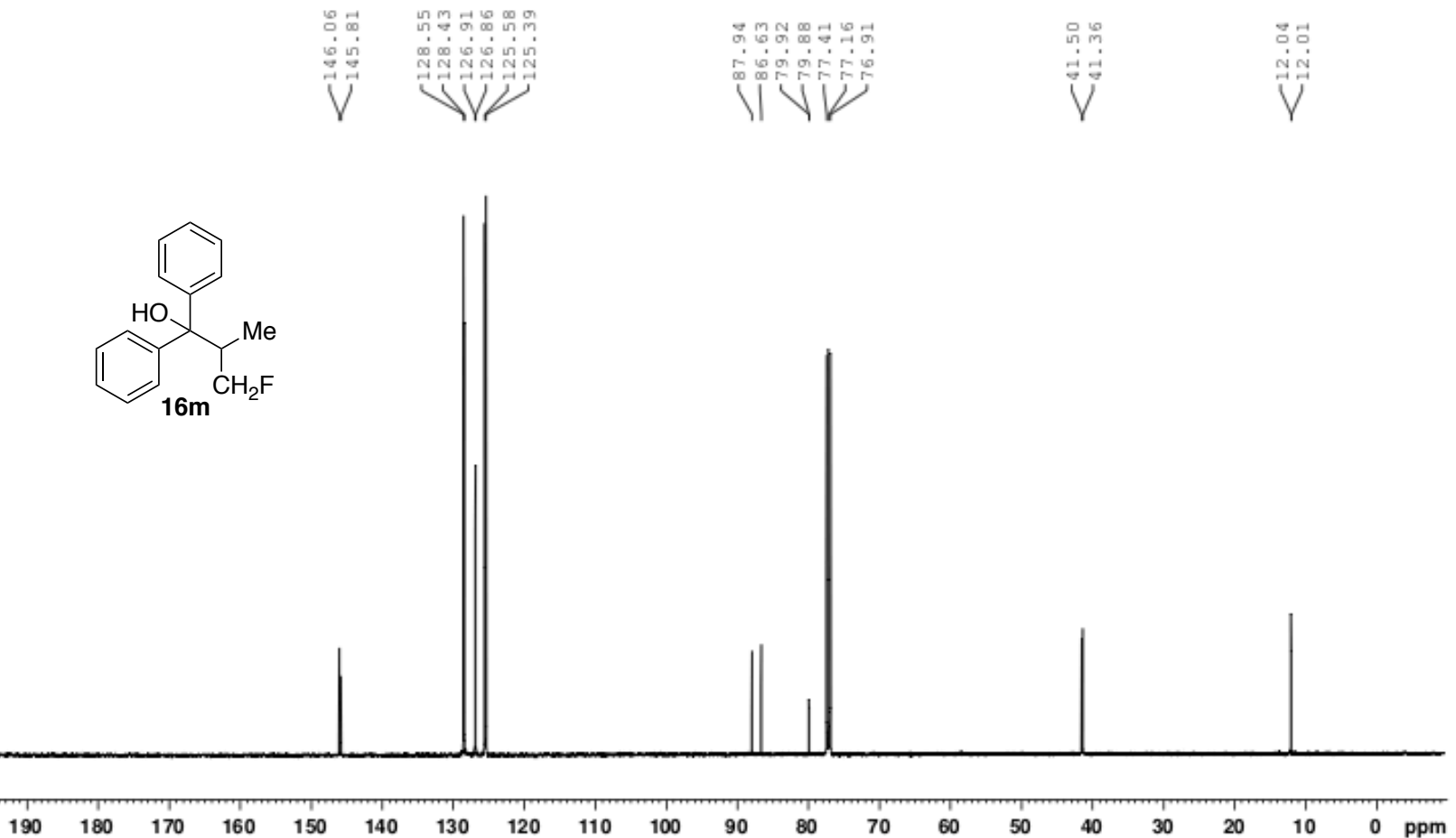
${ }^{19} \mathrm{~F} \mathrm{NMR}\left(376 \mathrm{MHz}, \mathrm{CDCl}_{3}, \mathrm{rt}\right)$
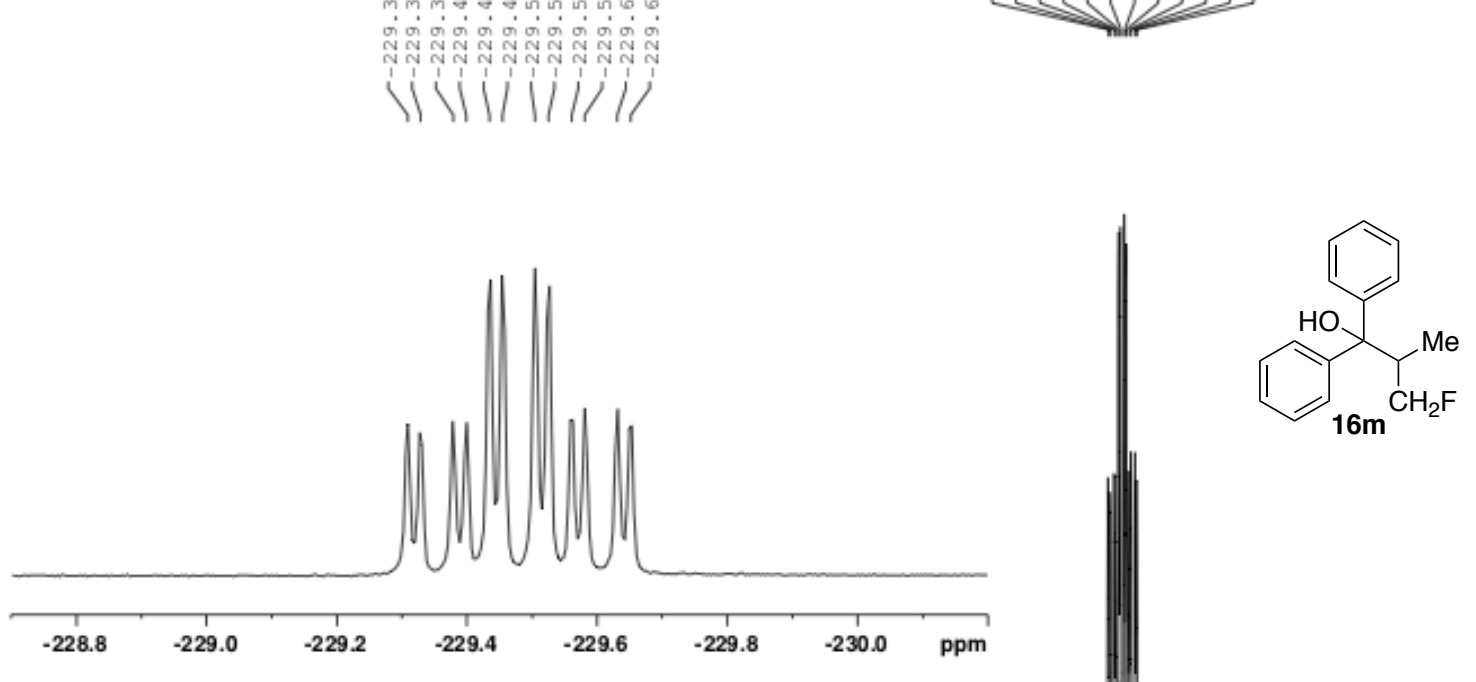

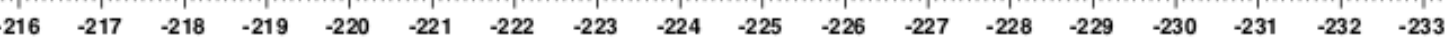

$\left.{ }^{1} \mathrm{H} \mathrm{NMR} \mathrm{(400} \mathrm{MHz,} \mathrm{CDCl}_{3}, \mathrm{rt}\right)$

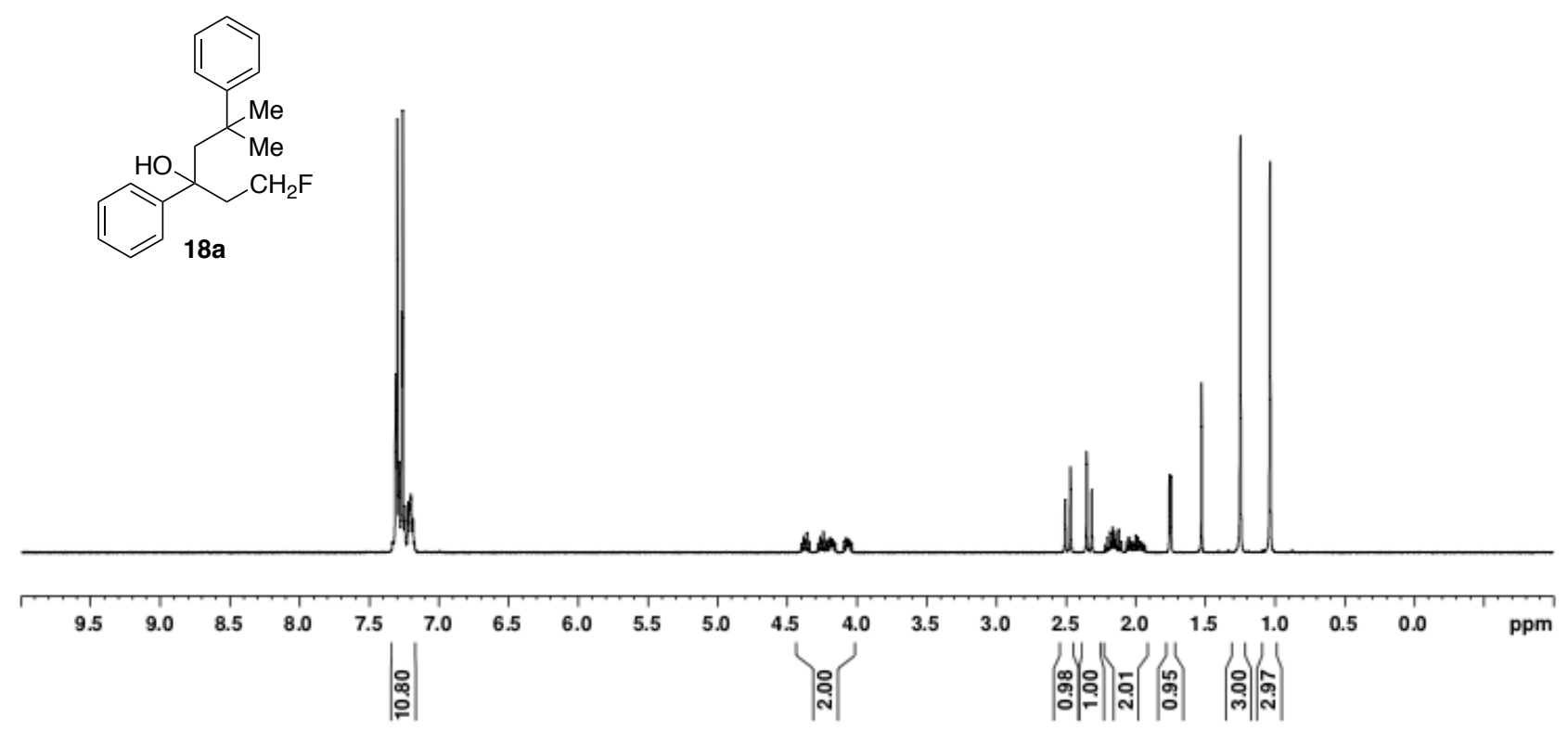


${ }^{13} \mathrm{C}$ NMR $\left(125 \mathrm{MHz}, \mathrm{CDCl}_{3}, \mathrm{rt}\right)$
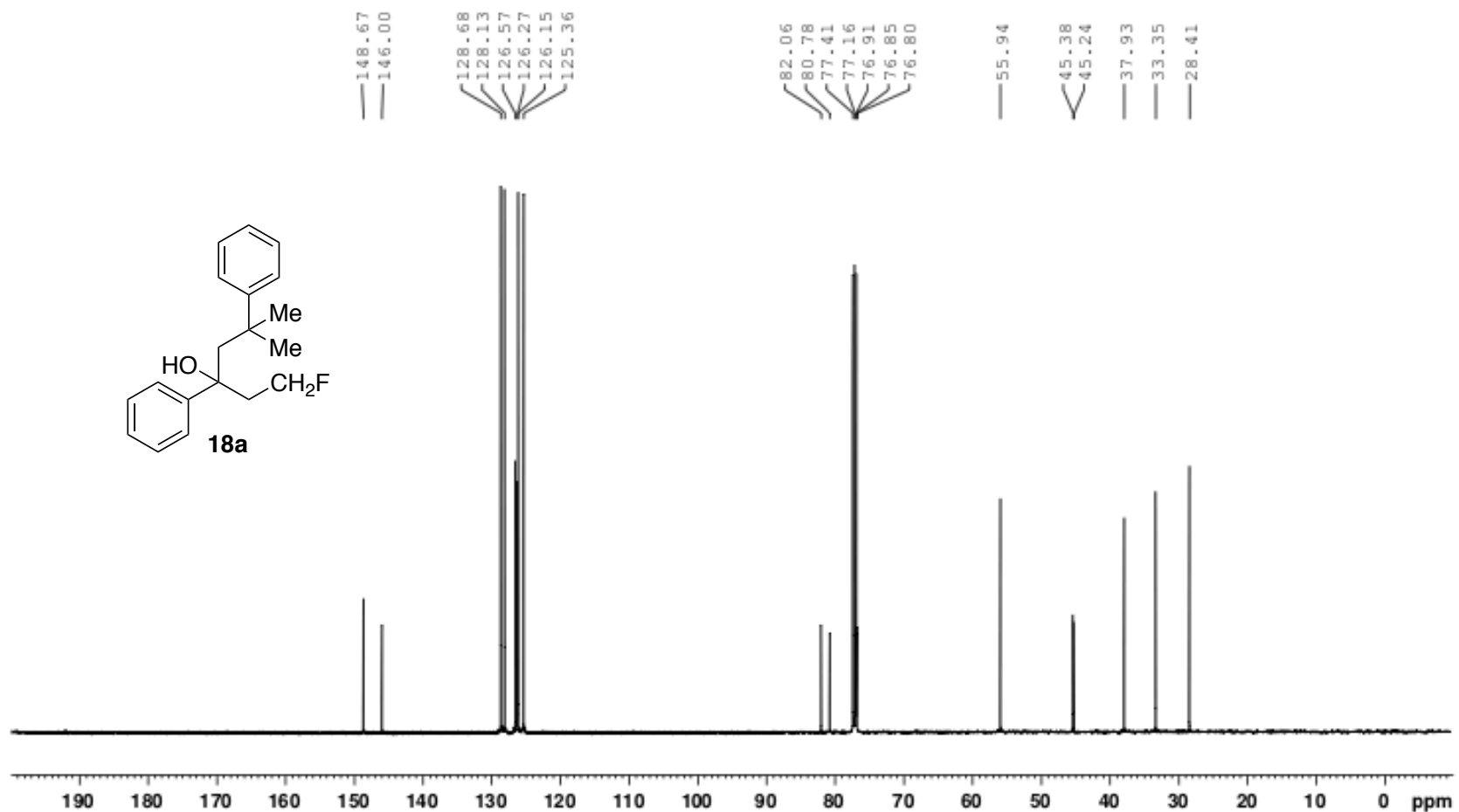

$\left.{ }^{19} \mathrm{~F} \mathrm{NMR} \mathrm{(376} \mathrm{MHz,} \mathrm{CDCl}_{3}, \mathrm{rt}\right)$

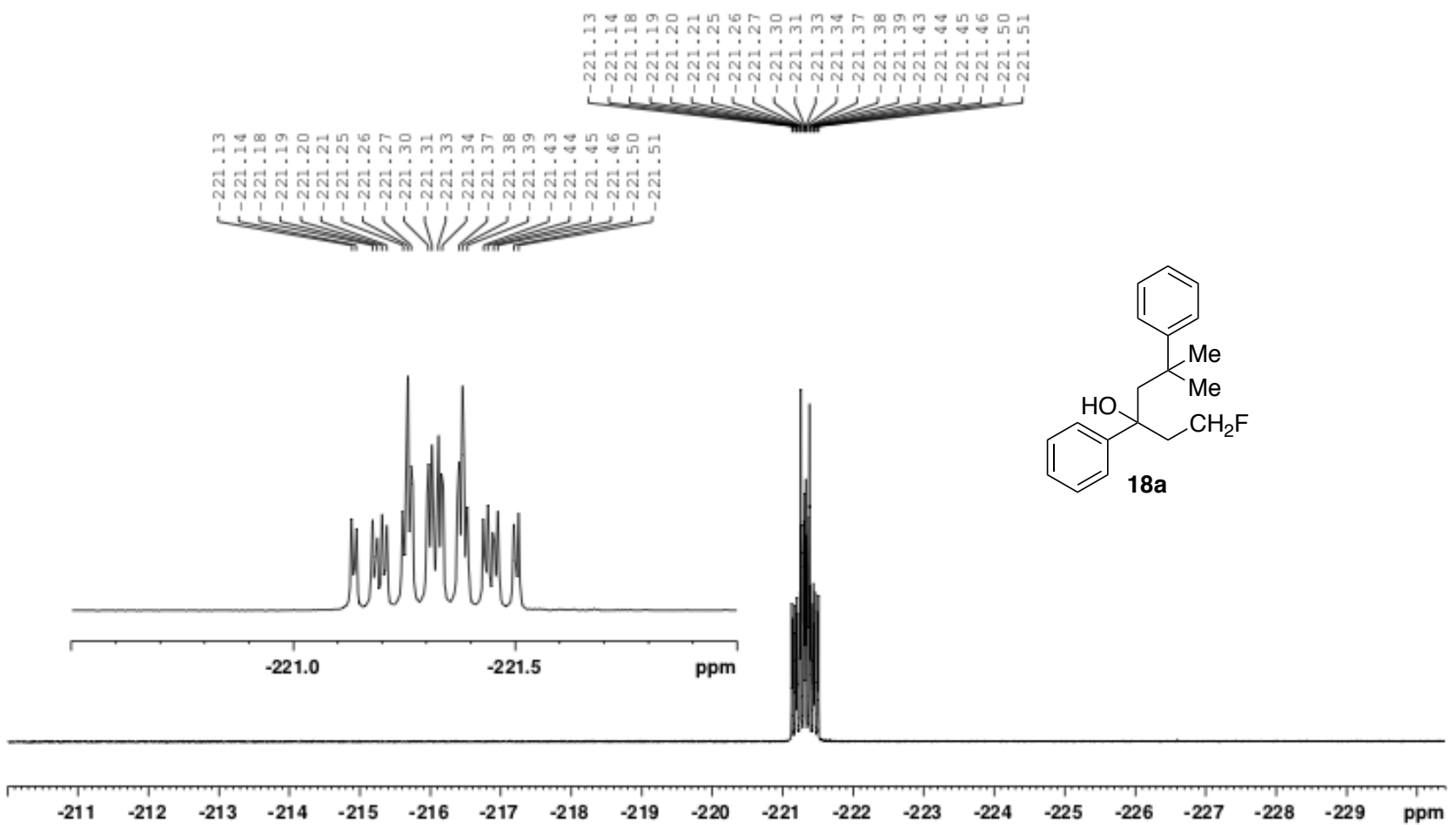


${ }^{1} \mathrm{H} \mathrm{NMR}\left(400 \mathrm{MHz}, \mathrm{CDCl}_{3}, \mathrm{rt}\right)$<smiles>CC(O)(CCF)c1ccccc1</smiles>

$18 b$

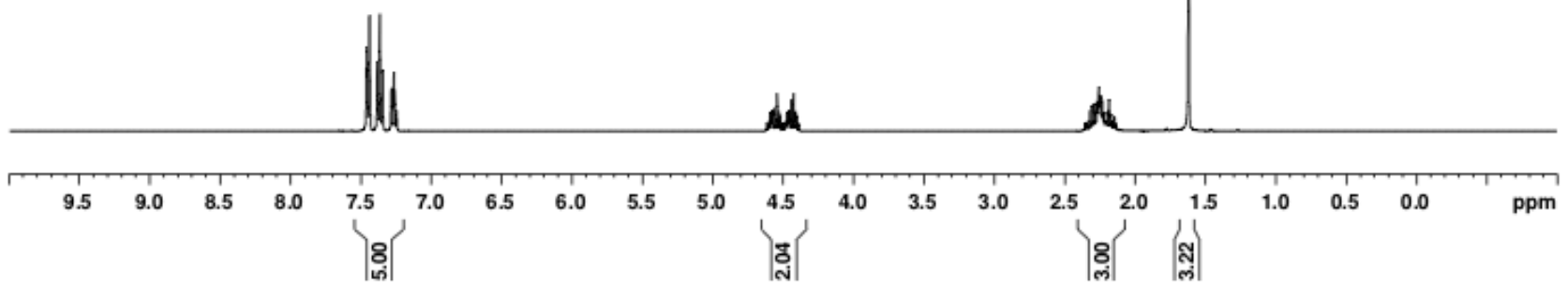

${ }^{13} \mathrm{C}$ NMR $\left(125 \mathrm{MHz}, \mathrm{CDCl}_{3}, \mathrm{rt}\right)$
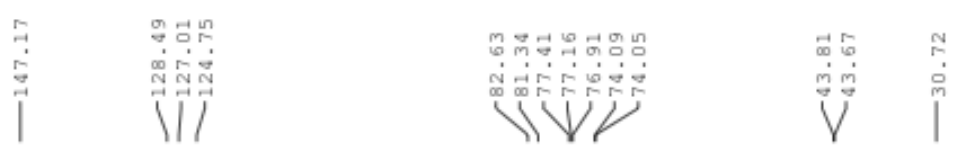<smiles>CC(C)(O)c1ccccc1</smiles>

$18 b$

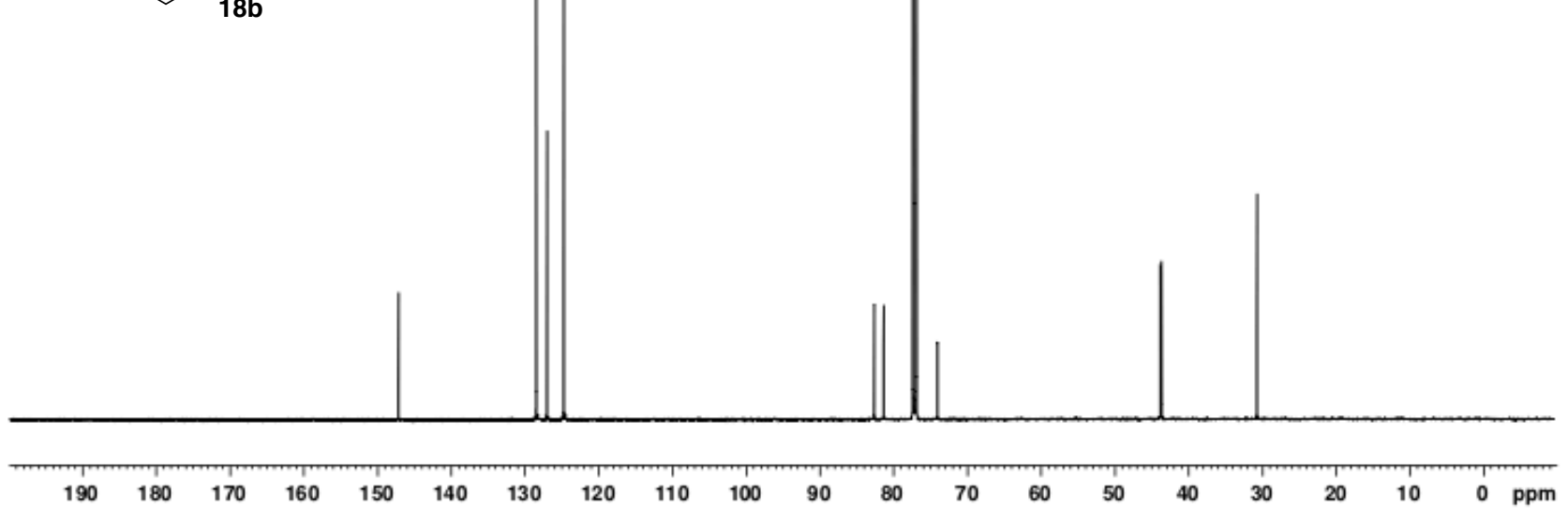


${ }^{19} \mathrm{~F} \mathrm{NMR}\left(376 \mathrm{MHz}, \mathrm{CDCl}_{3}, \mathrm{rt}\right)$

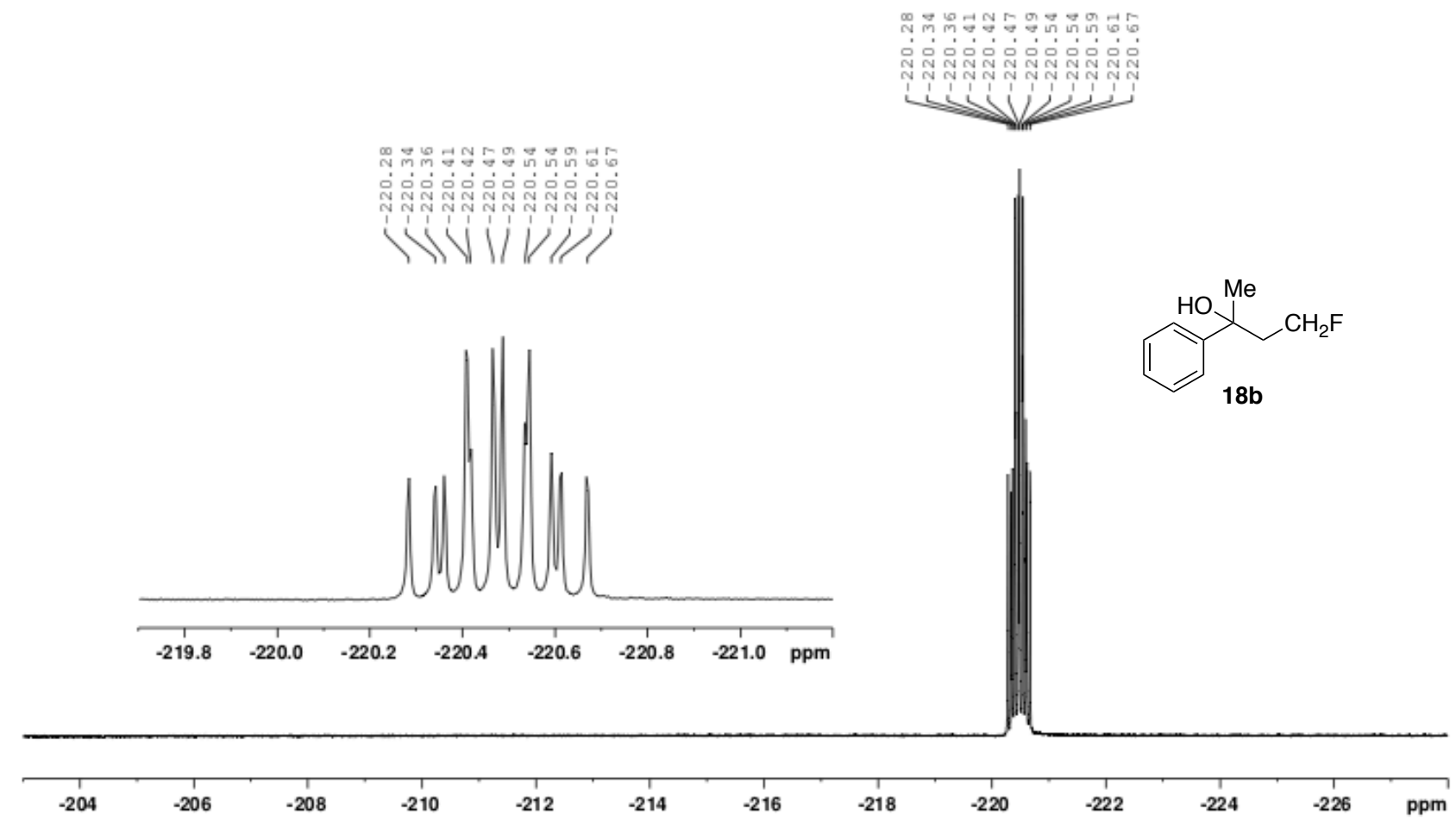

${ }^{1} \mathrm{H}$ NMR $\left(400 \mathrm{MHz}, \mathrm{CDCl}_{3}, \mathrm{rt}\right)$

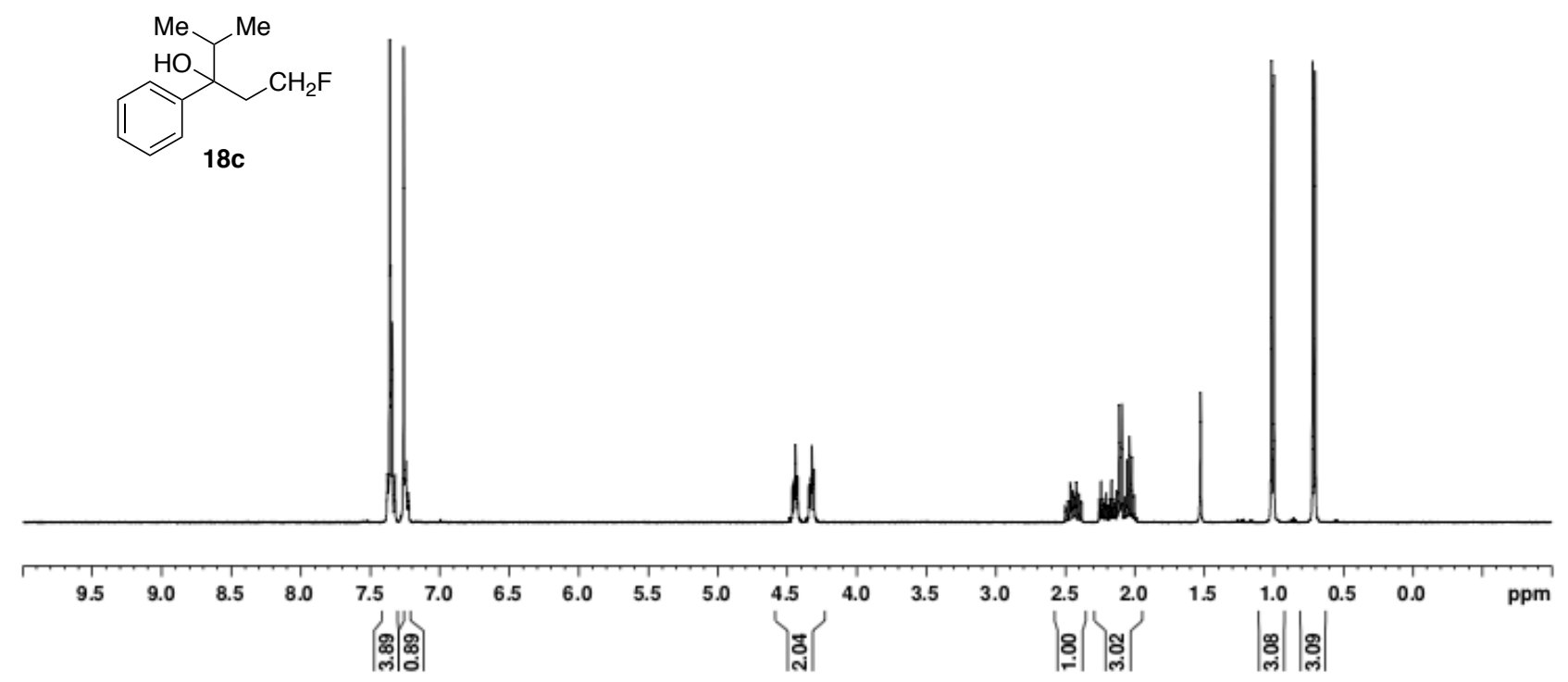


${ }^{13} \mathrm{C}$ NMR $\left(125 \mathrm{MHz}, \mathrm{CDCl}_{3}, \mathrm{rt}\right)$
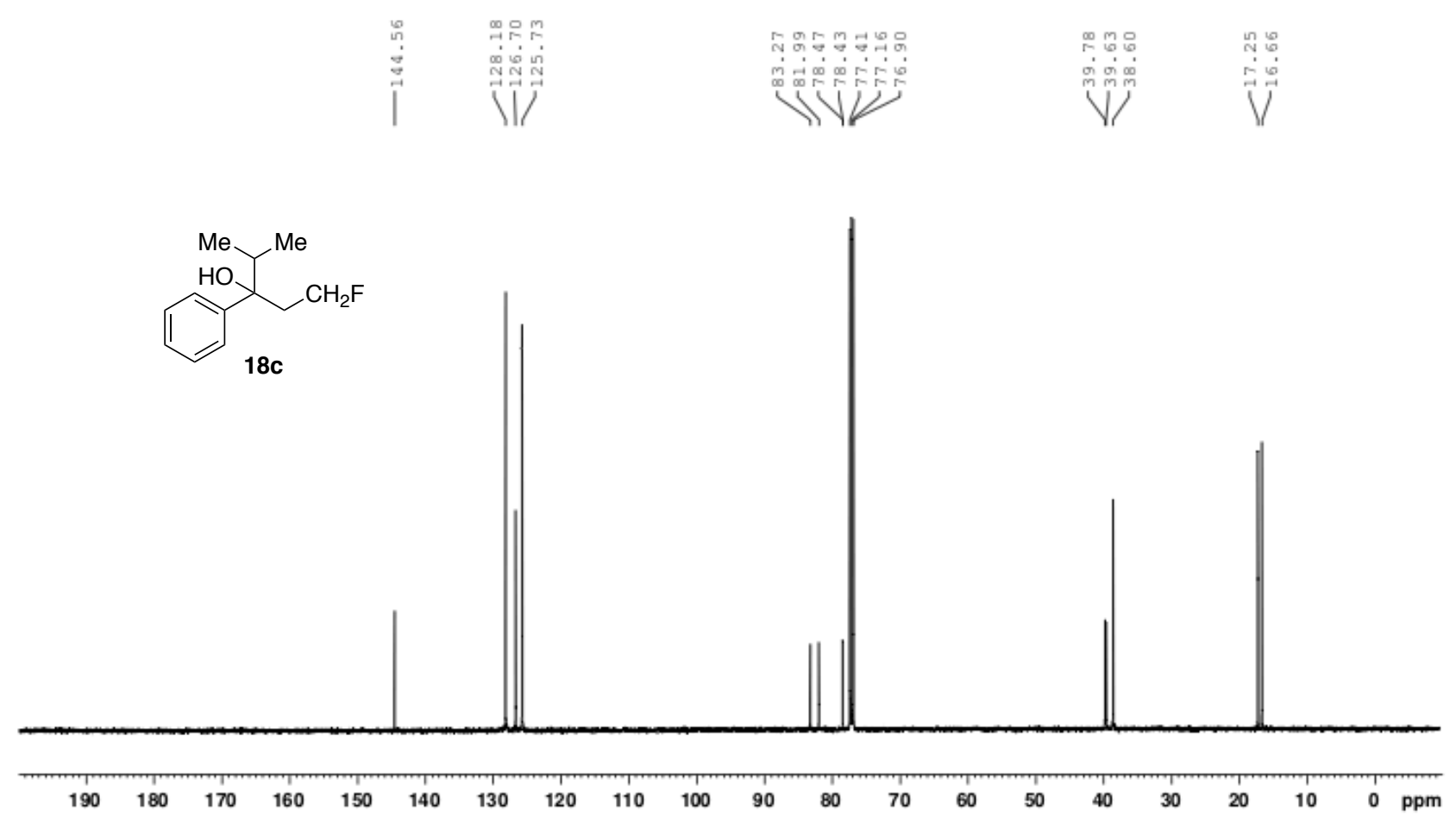

${ }^{19} \mathrm{~F} \mathrm{NMR}\left(376 \mathrm{MHz}, \mathrm{CDCl}_{3}, \mathrm{rt}\right)$

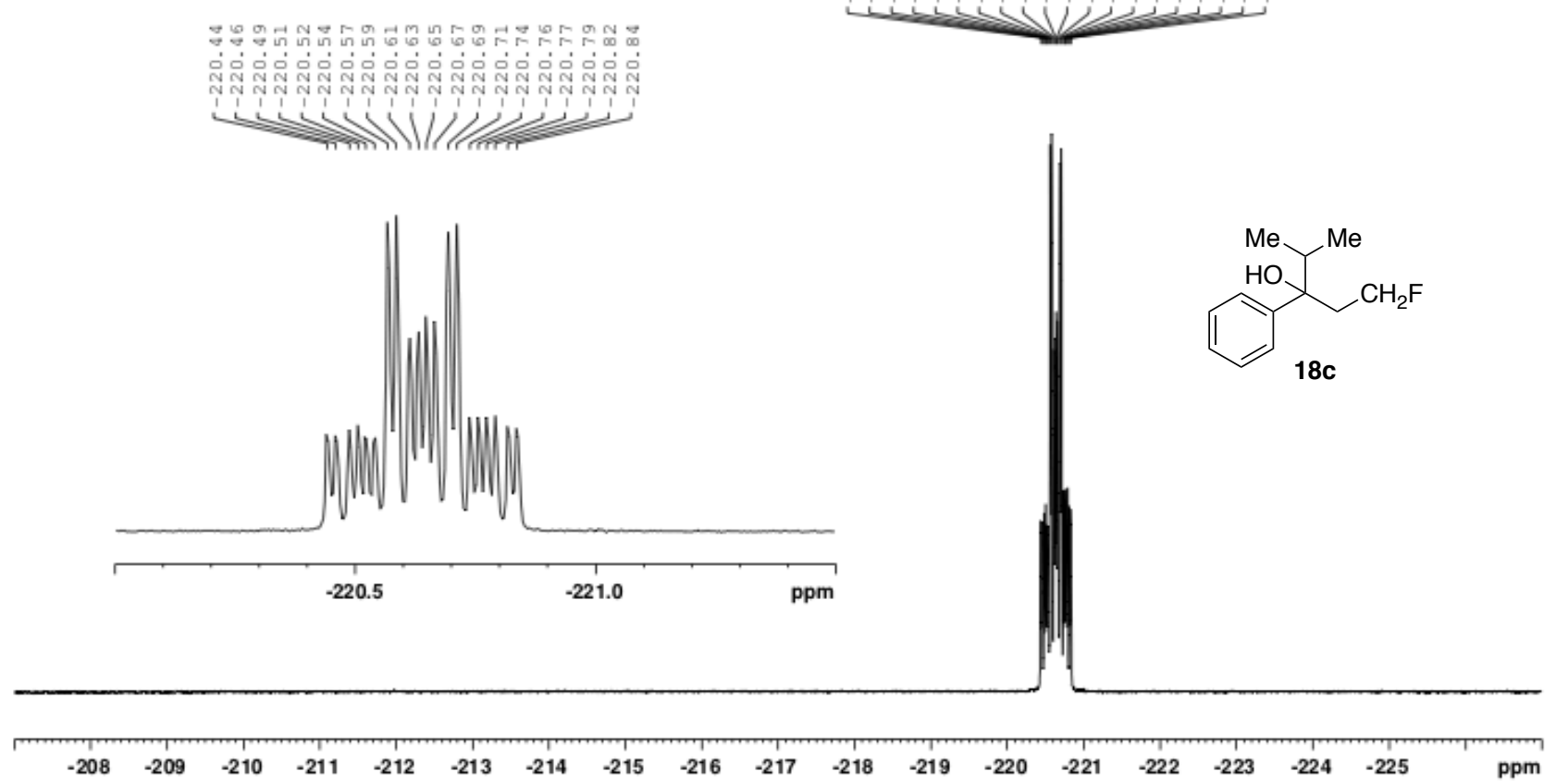


${ }^{1} \mathrm{H} \mathrm{NMR}\left(400 \mathrm{MHz}, \mathrm{CDCl}_{3}, \mathrm{rt}\right)$
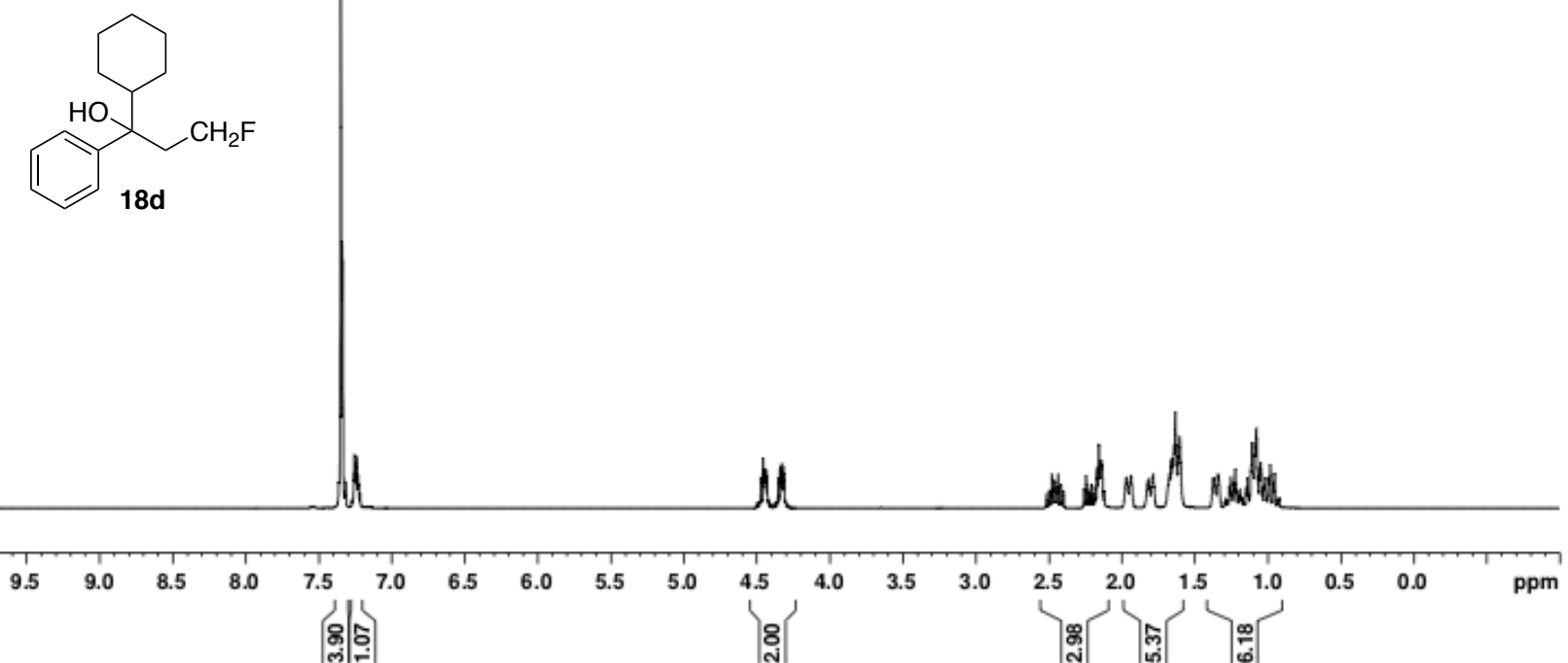

${ }^{13} \mathrm{C}$ NMR $\left(125 \mathrm{MHz}, \mathrm{CDCl}_{3}, \mathrm{rt}\right)$
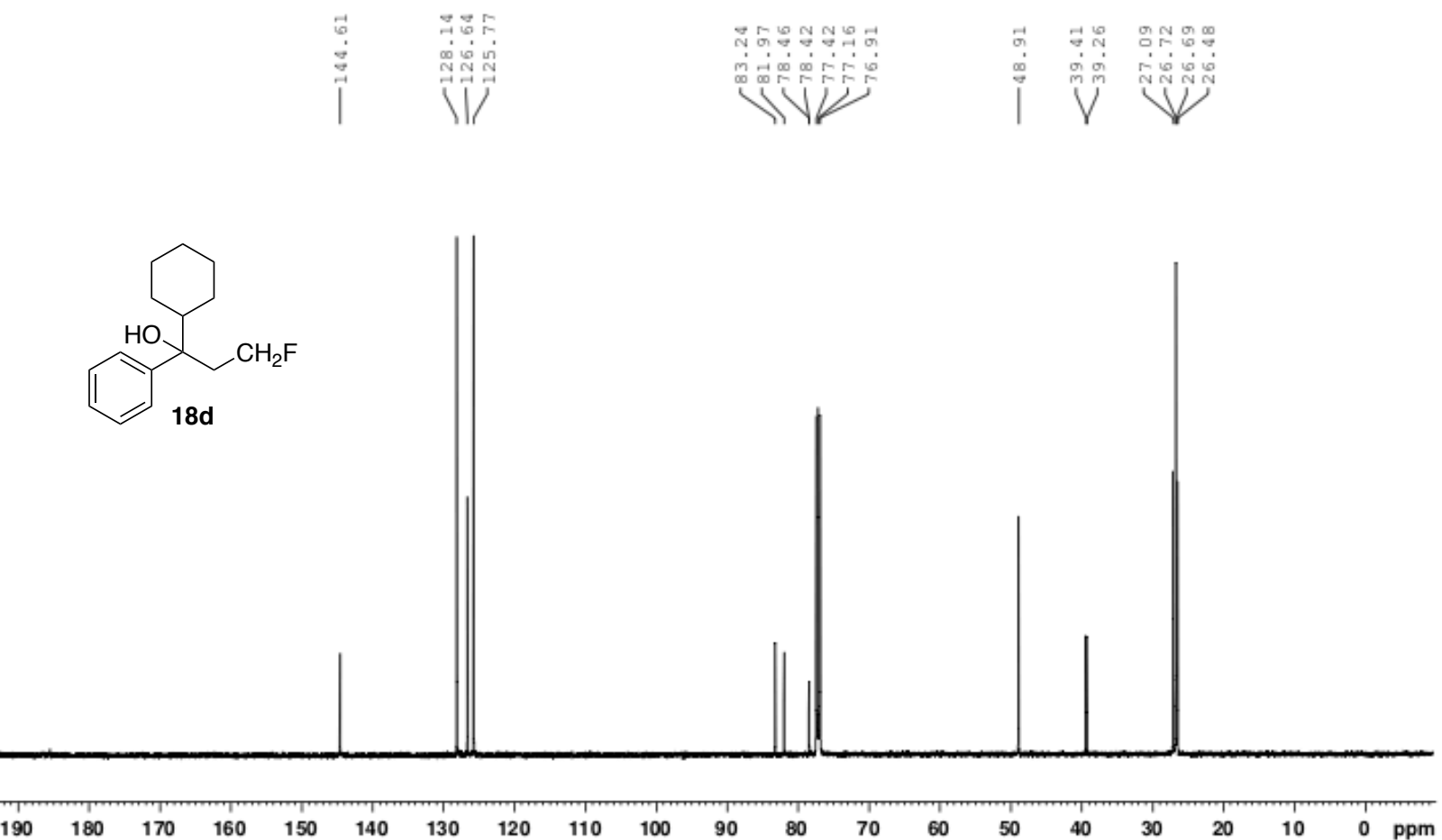
${ }^{19} \mathrm{~F} \mathrm{NMR}\left(376 \mathrm{MHz}, \mathrm{CDCl}_{3}, \mathrm{rt}\right)$

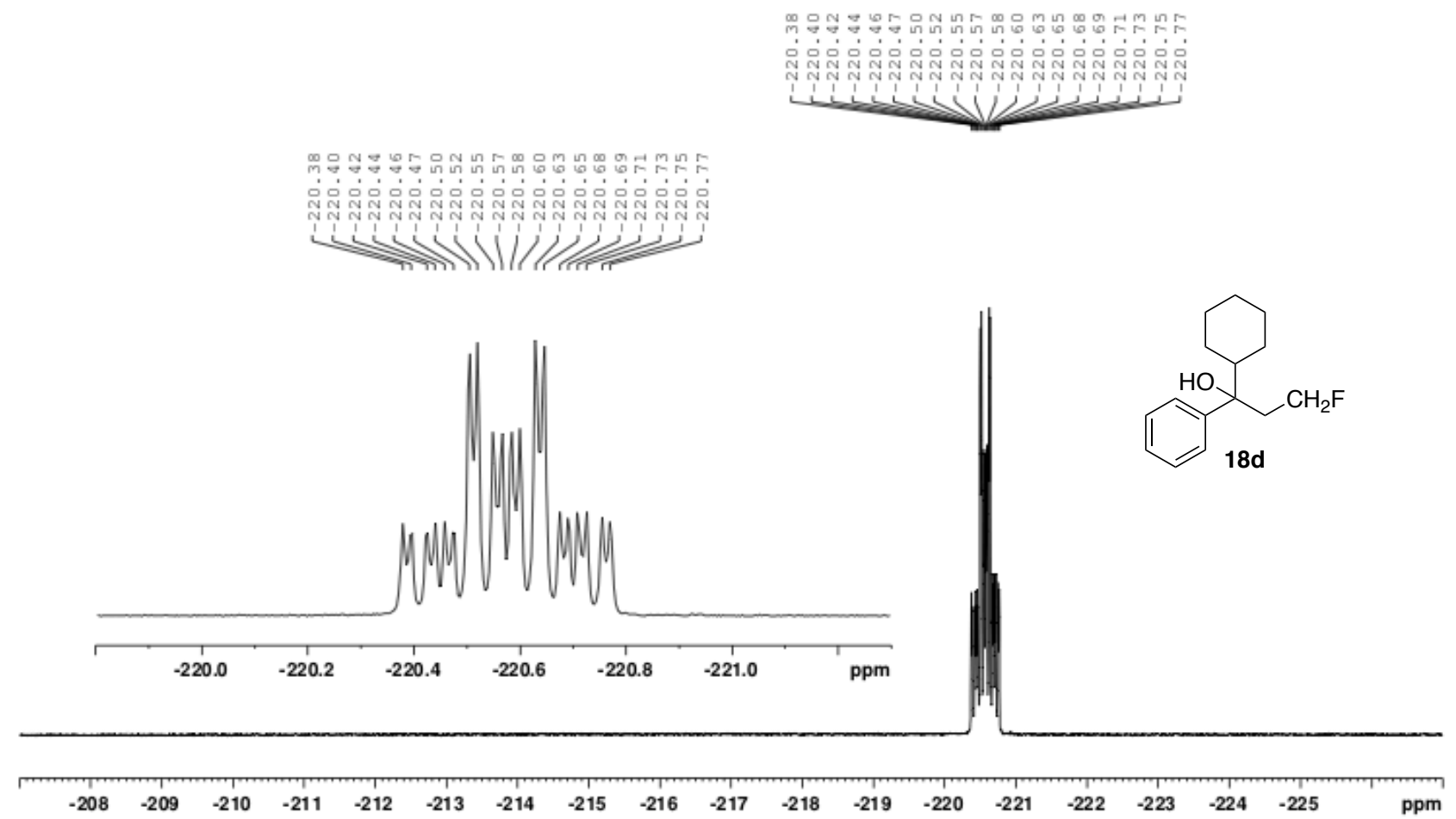

${ }^{1} \mathrm{H}$ NMR $\left(400 \mathrm{MHz}, \mathrm{CDCl}_{3}, \mathrm{rt}\right)$

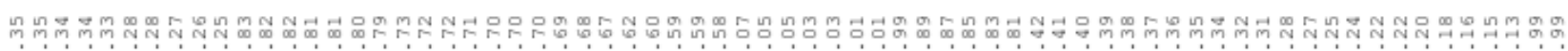

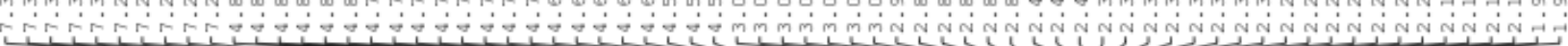

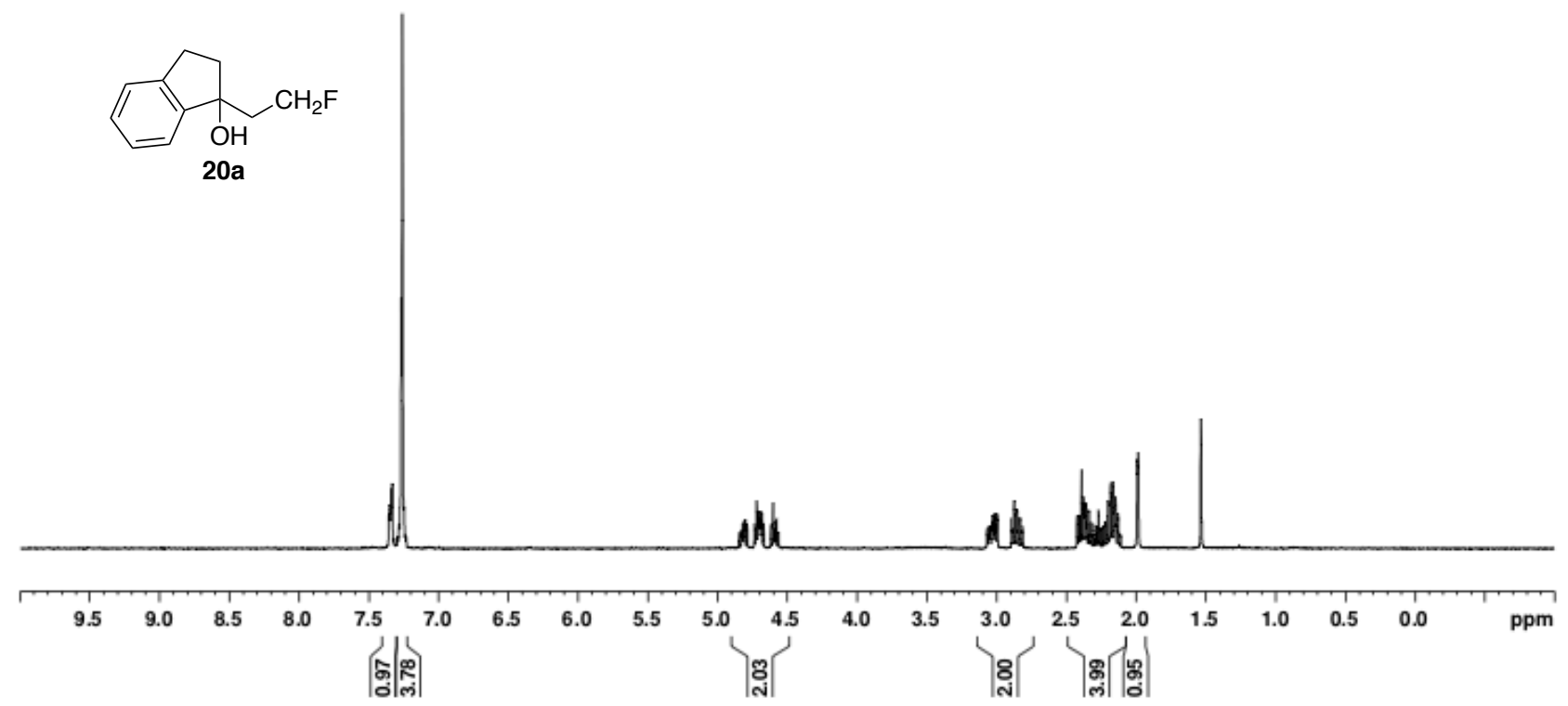


${ }^{13} \mathrm{C}$ NMR $\left(125 \mathrm{MHz}, \mathrm{CDCl}_{3}, \mathrm{rt}\right)$

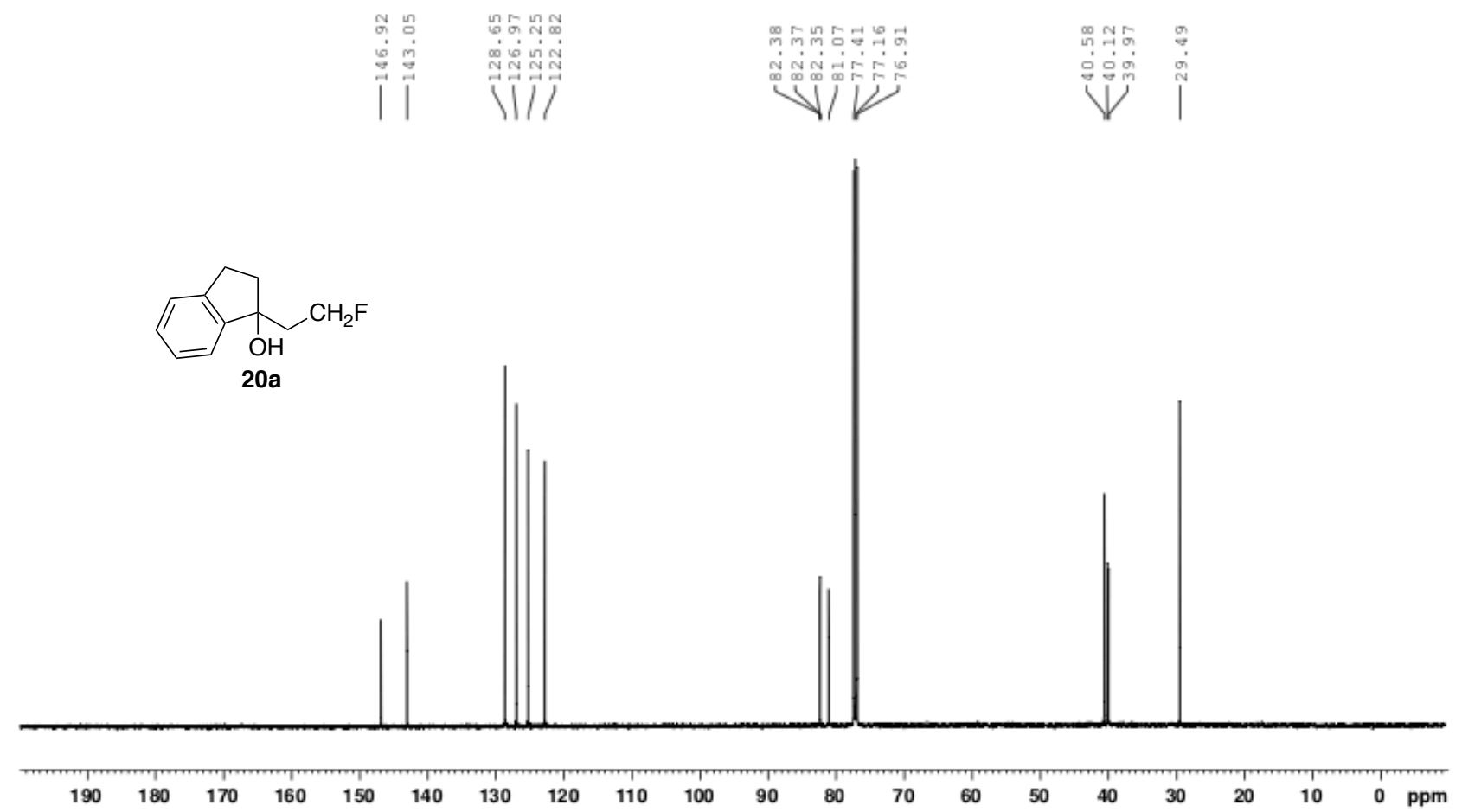

${ }^{19} \mathrm{~F} \mathrm{NMR}\left(376 \mathrm{MHz}, \mathrm{CDCl}_{3}, \mathrm{rt}\right)$

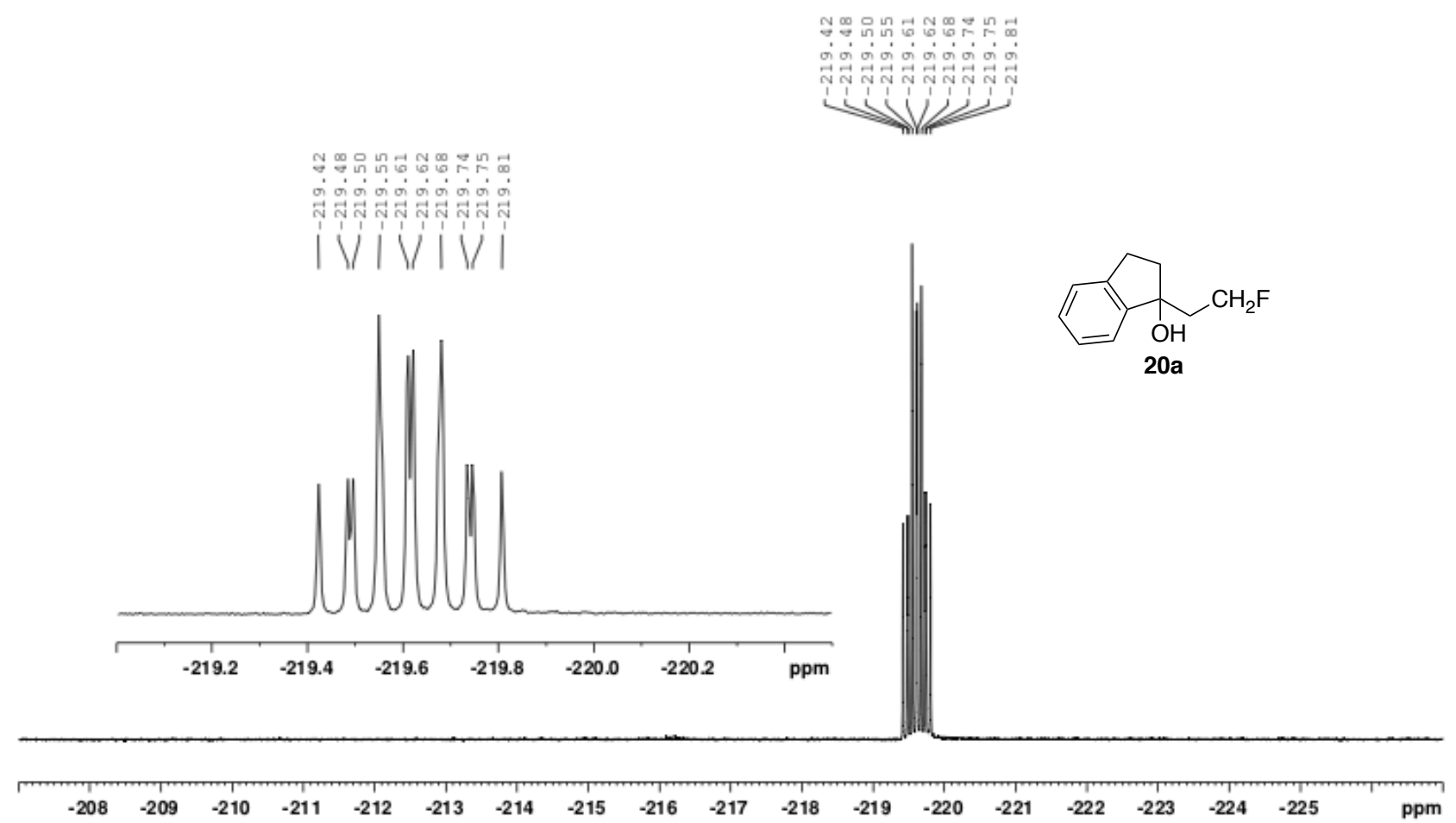


${ }^{1} \mathrm{H} \mathrm{NMR}\left(400 \mathrm{MHz}, \mathrm{CDCl}_{3}, \mathrm{rt}\right)$

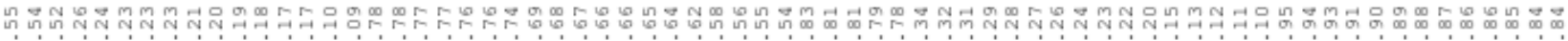

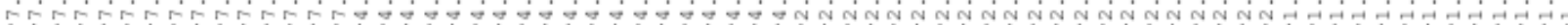<smiles>OC1(CF)CCCc2ccccc21</smiles>

$20 \mathrm{~b}$

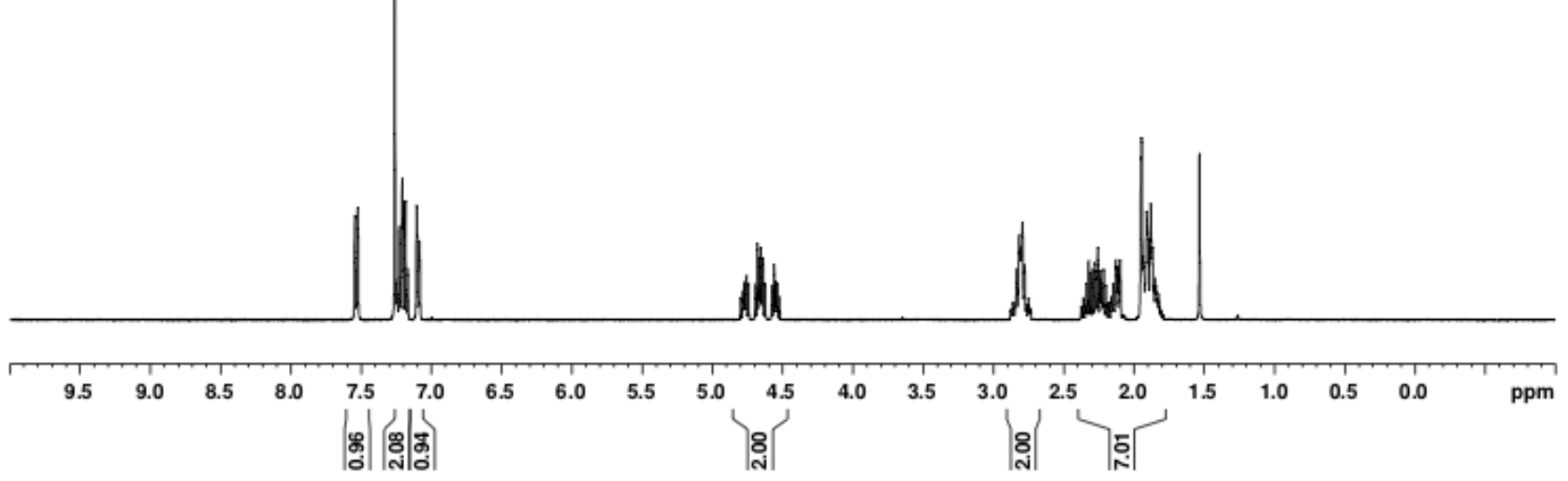

${ }^{13} \mathrm{C} \mathrm{NMR}\left(125 \mathrm{MHz}, \mathrm{CDCl}_{3}, \mathrm{rt}\right)$
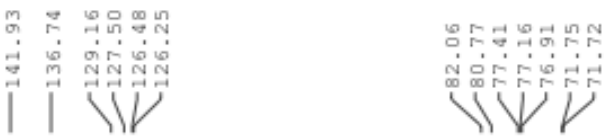

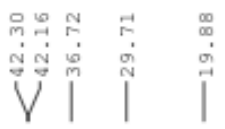<smiles>OC1(CCF)CCCc2ccccc21</smiles>

$20 \mathrm{~b}$

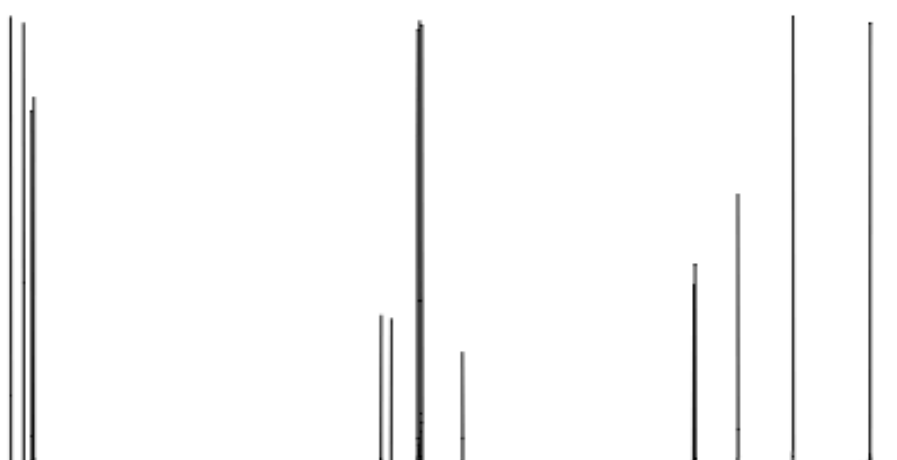

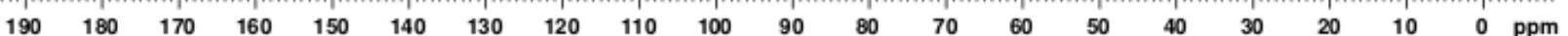


${ }^{19} \mathrm{~F} \mathrm{NMR}\left(376 \mathrm{MHz}, \mathrm{CDCl}_{3}, \mathrm{rt}\right)$

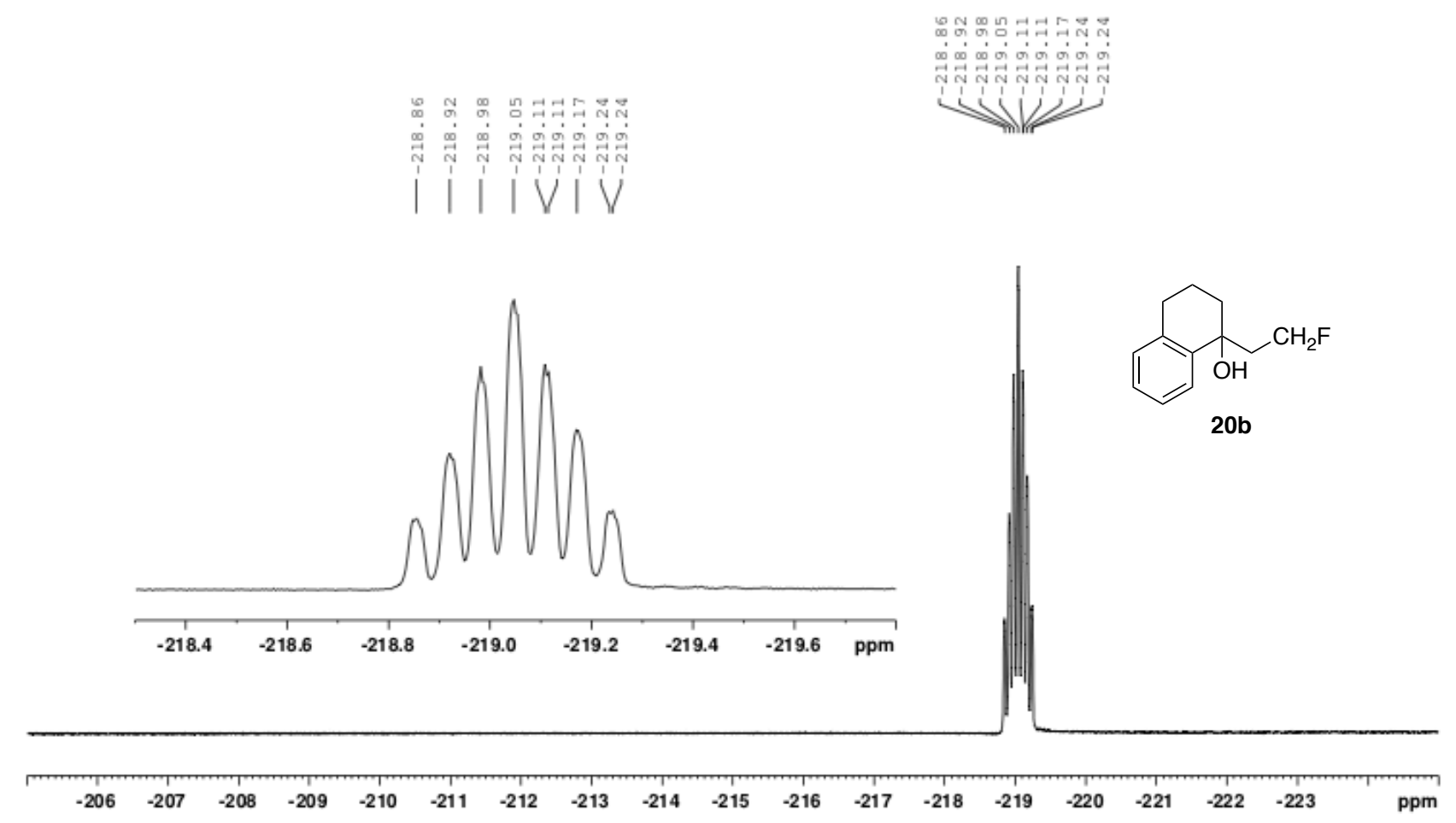

$\left.{ }^{1} \mathrm{H} \mathrm{NMR} \mathrm{(400} \mathrm{MHz,} \mathrm{CDCl}_{3}, \mathrm{rt}\right)$

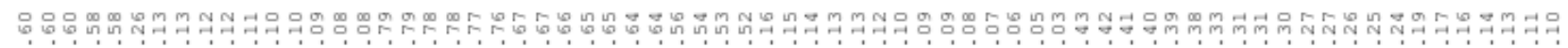

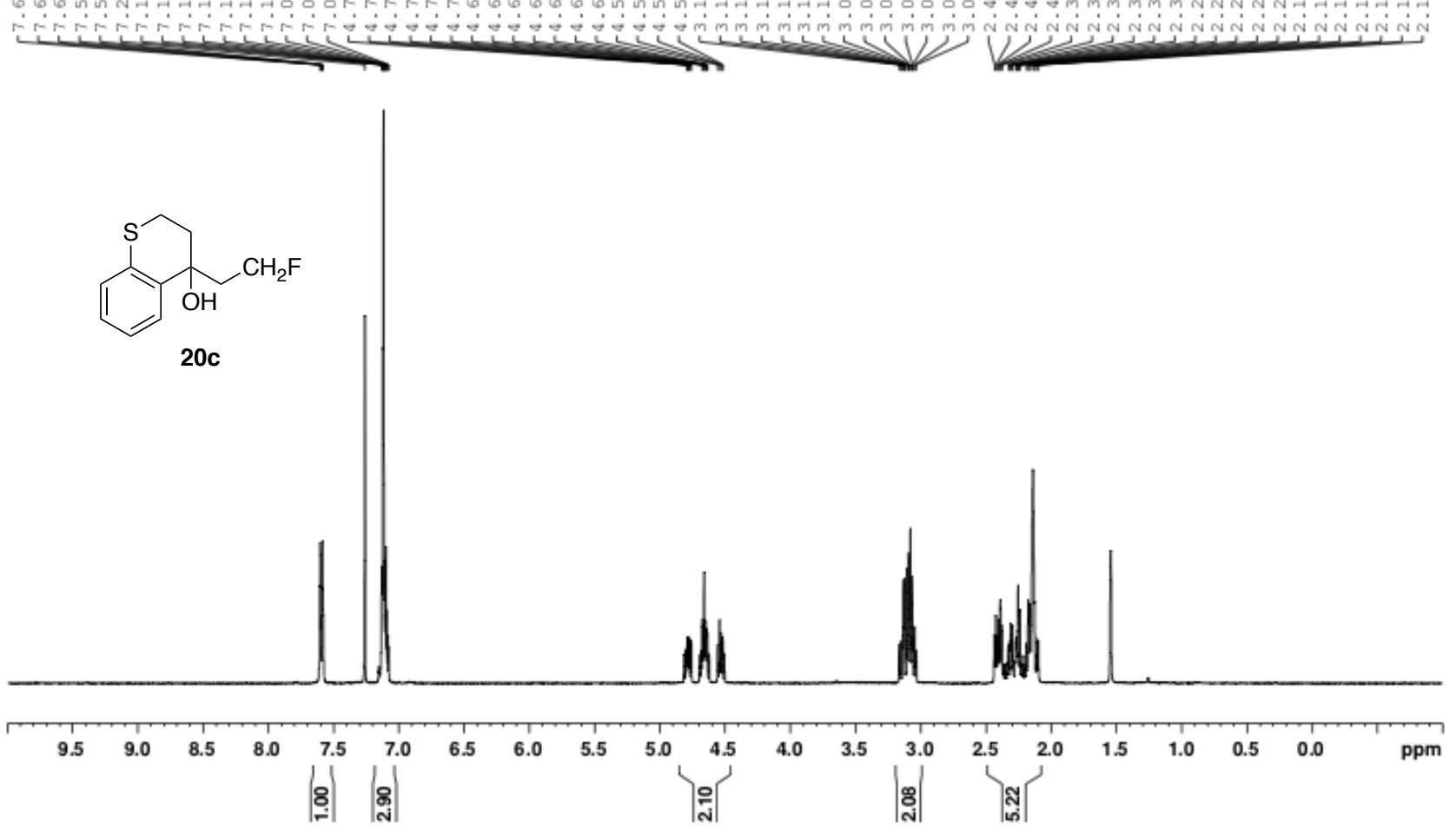


${ }^{13} \mathrm{C}$ NMR $\left(125 \mathrm{MHz}, \mathrm{CDCl}_{3}, \mathrm{rt}\right)$

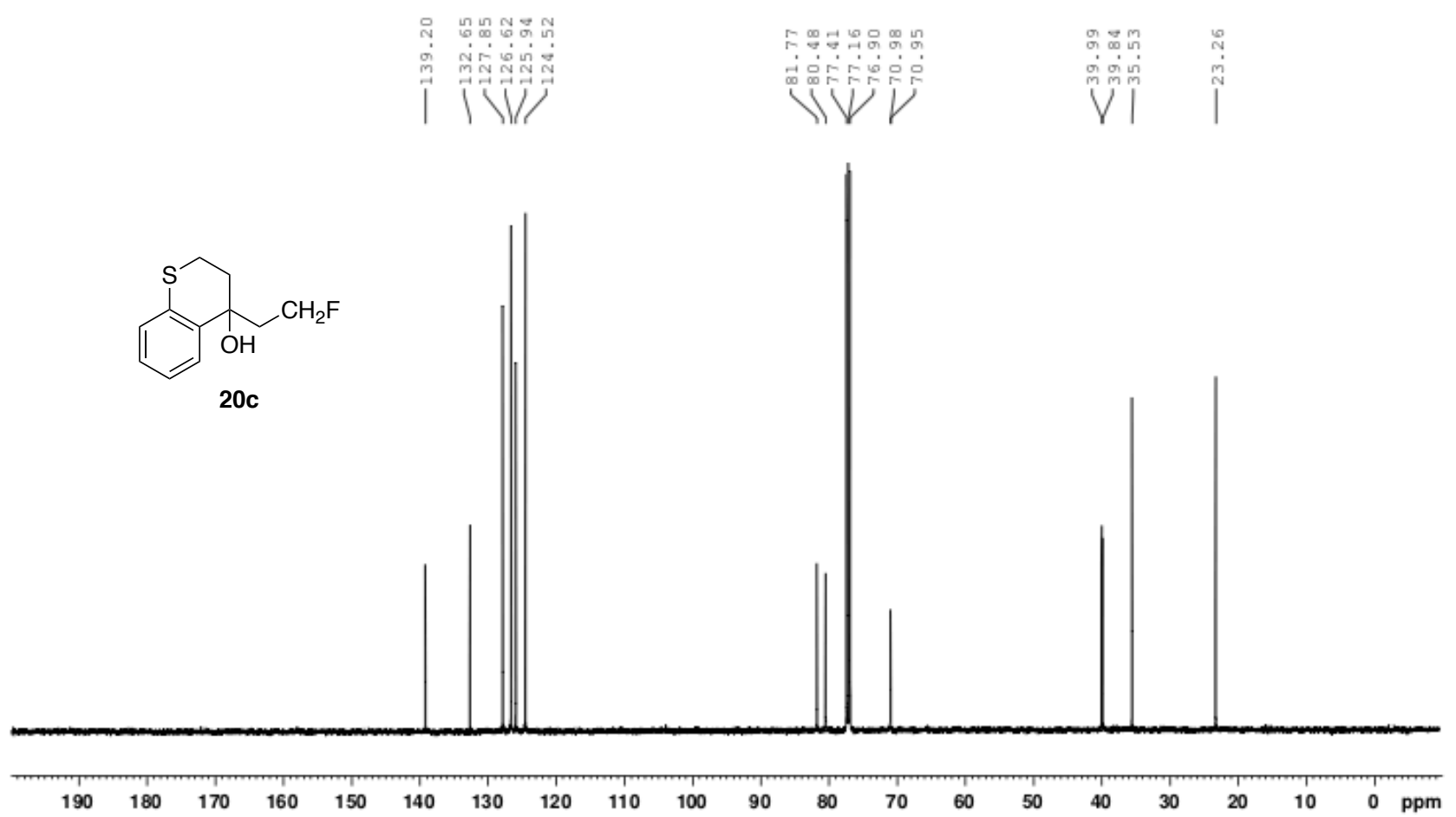

${ }^{19} \mathrm{~F} \mathrm{NMR}\left(376 \mathrm{MHz}, \mathrm{CDCl}_{3}, \mathrm{rt}\right)$

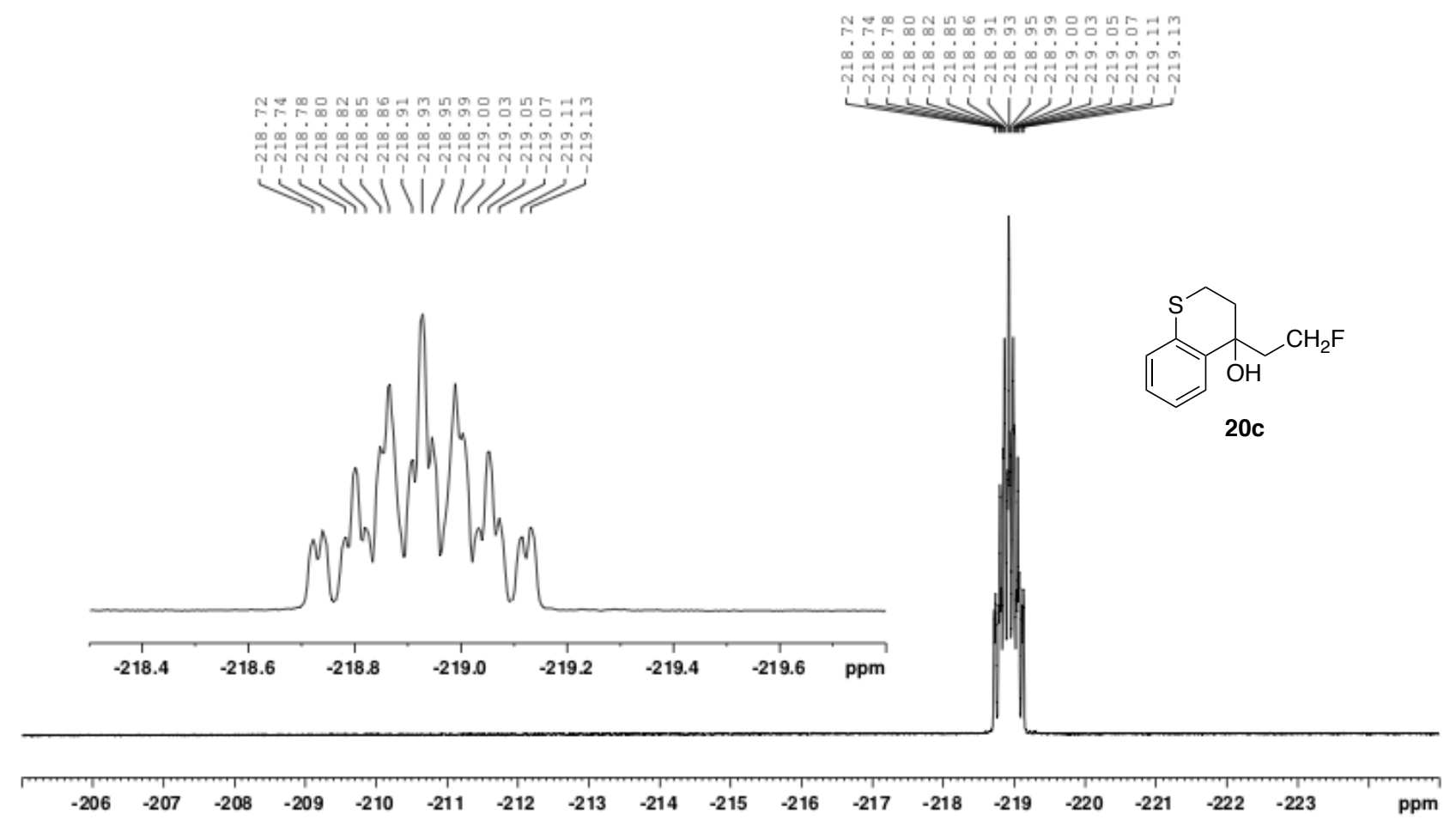


${ }^{1} \mathrm{H}$ NMR $\left(400 \mathrm{MHz}, \mathrm{CDCl}_{3}, \mathrm{rt}\right)$
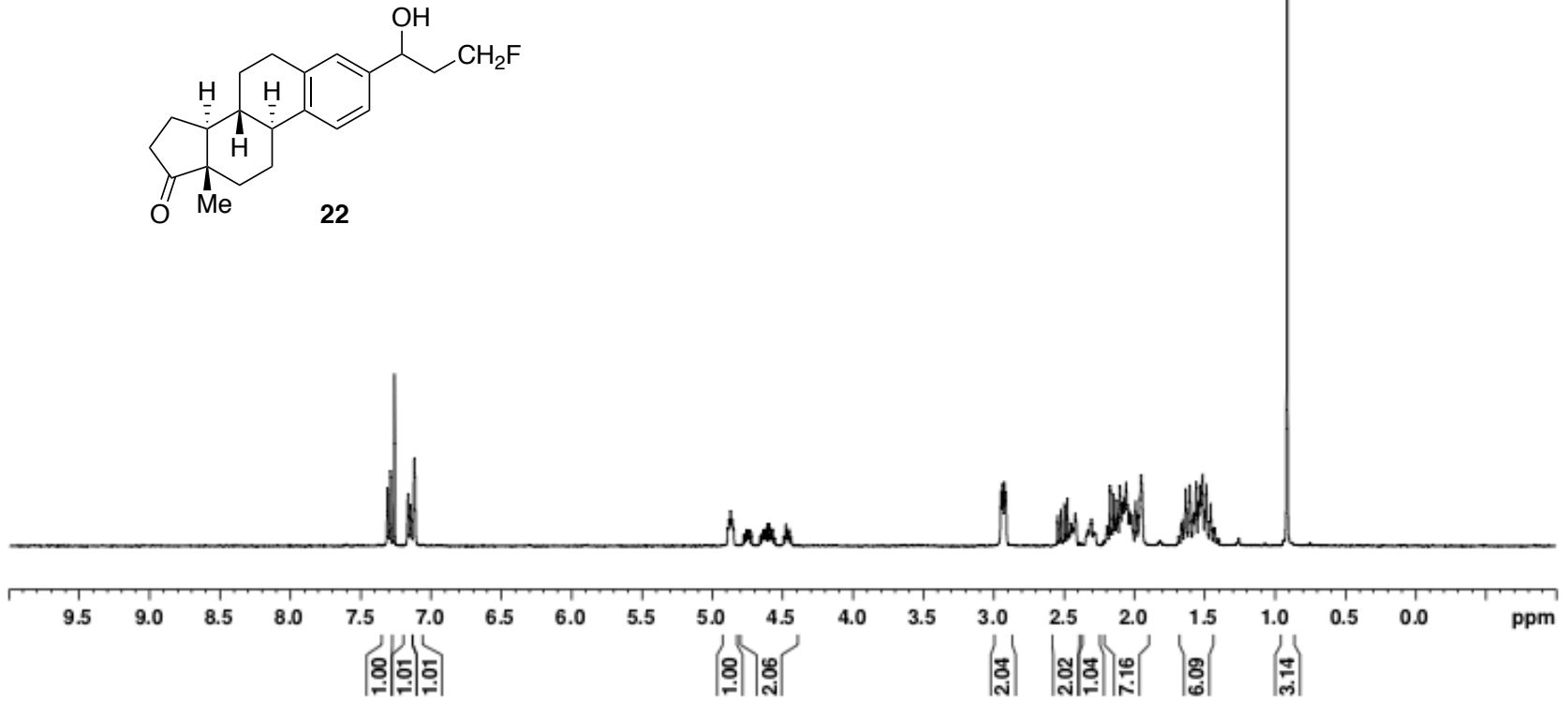

${ }^{13} \mathrm{C}$ NMR $\left(125 \mathrm{MHz}, \mathrm{CDCl}_{3}, \mathrm{rt}\right)$

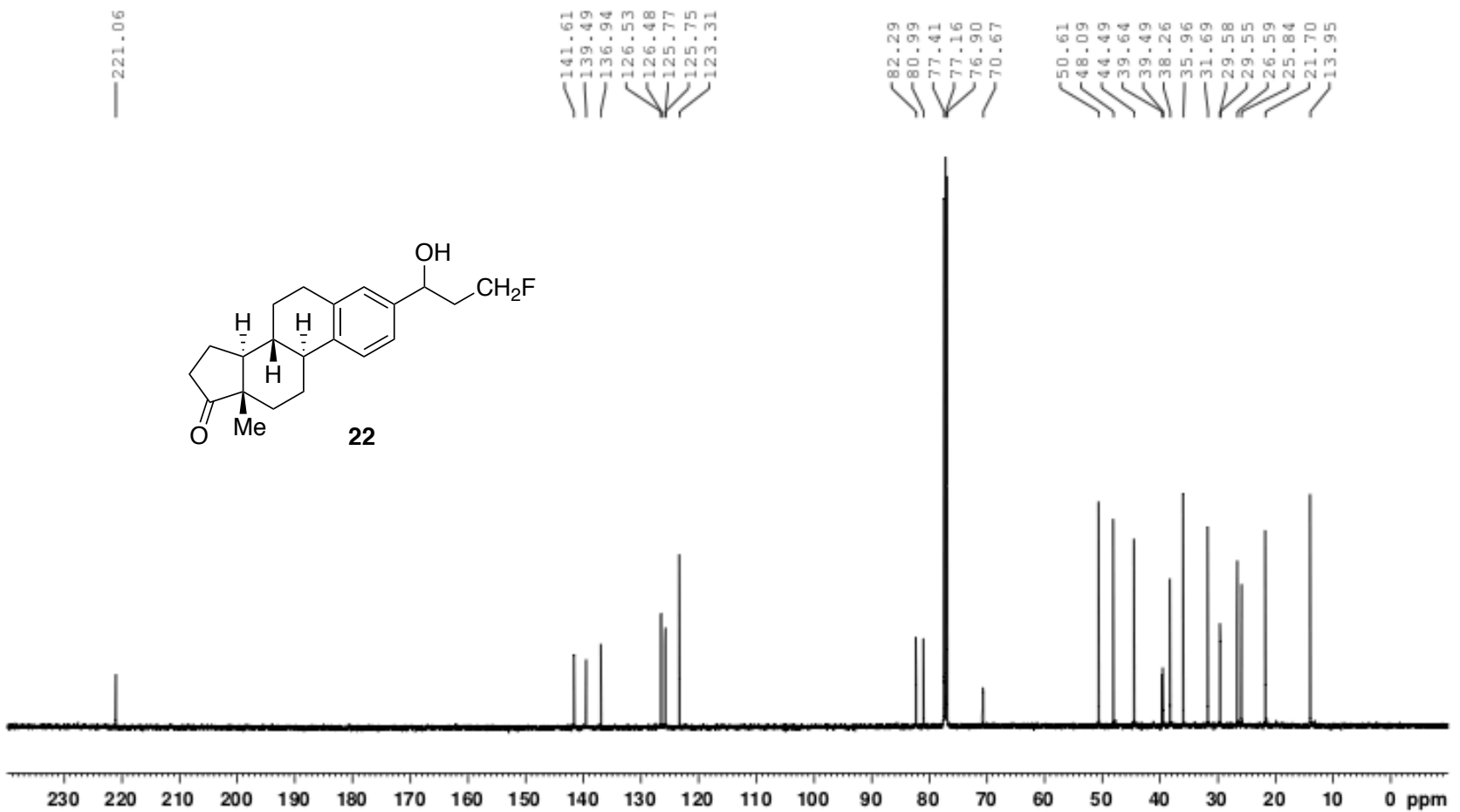


${ }^{19} \mathrm{~F} \mathrm{NMR}\left(376 \mathrm{MHz}, \mathrm{CDCl}_{3}, \mathrm{rt}\right)$

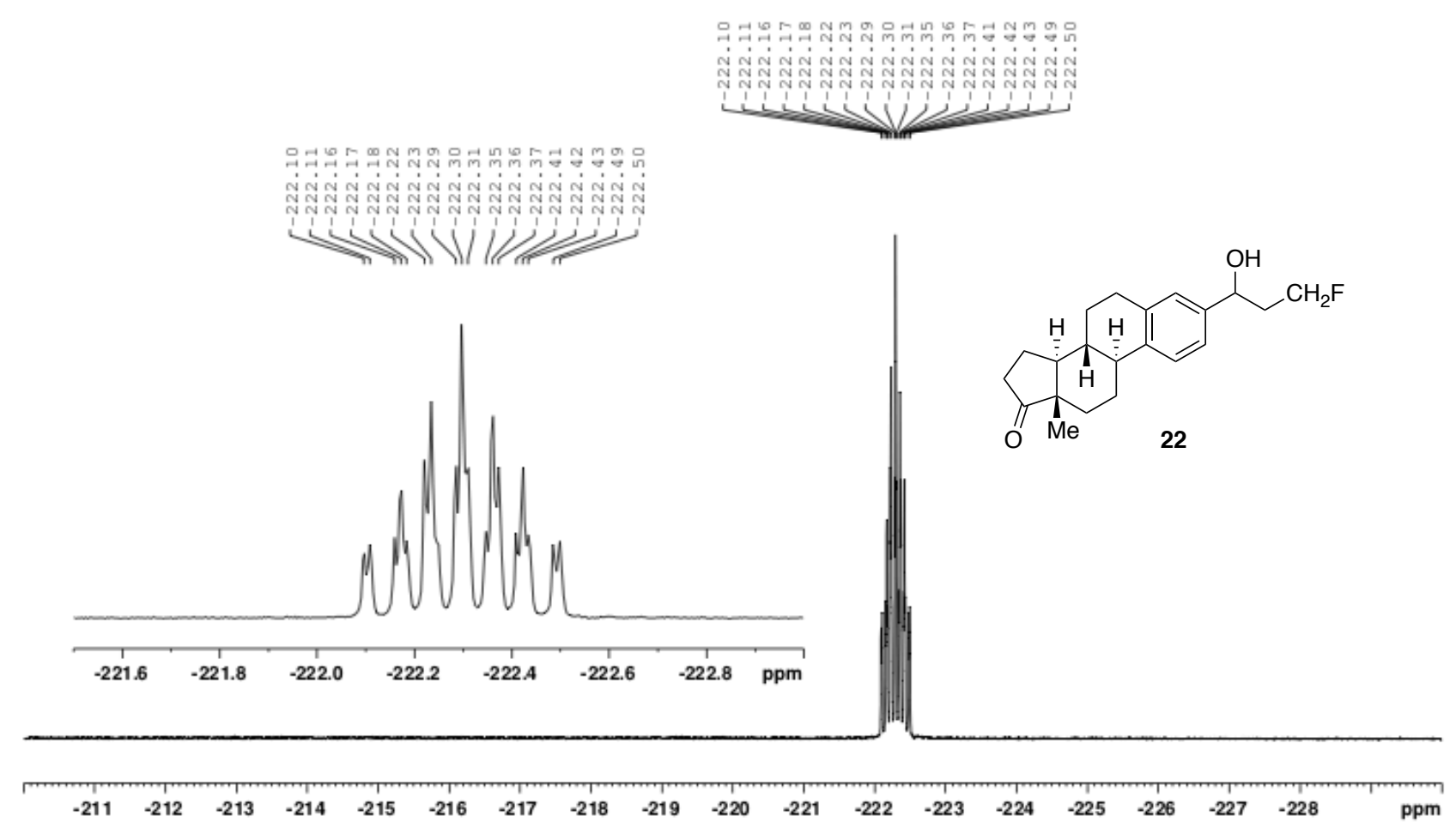

${ }^{1} \mathrm{H} \mathrm{NMR}\left(400 \mathrm{MHz}, \mathrm{CDCl}_{3}, \mathrm{rt}\right)$<smiles>CCC1(O)CC(c2ccccc2)Oc2ccccc21</smiles>

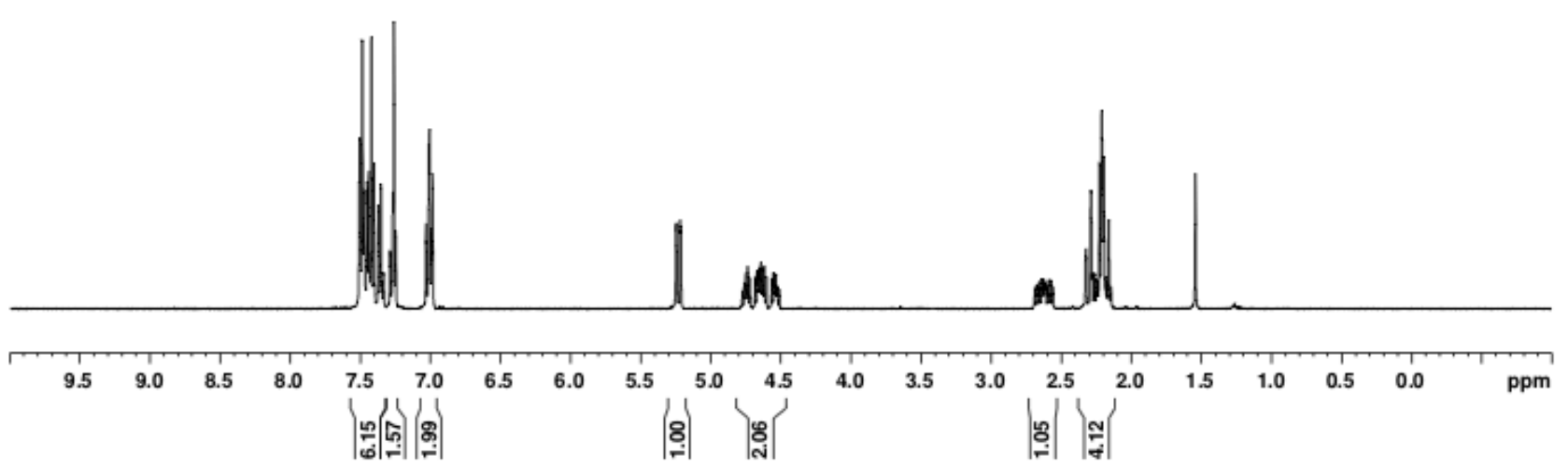


${ }^{13} \mathrm{C}$ NMR $\left(125 \mathrm{MHz}, \mathrm{CDCl}_{3}, \mathrm{rt}\right)$

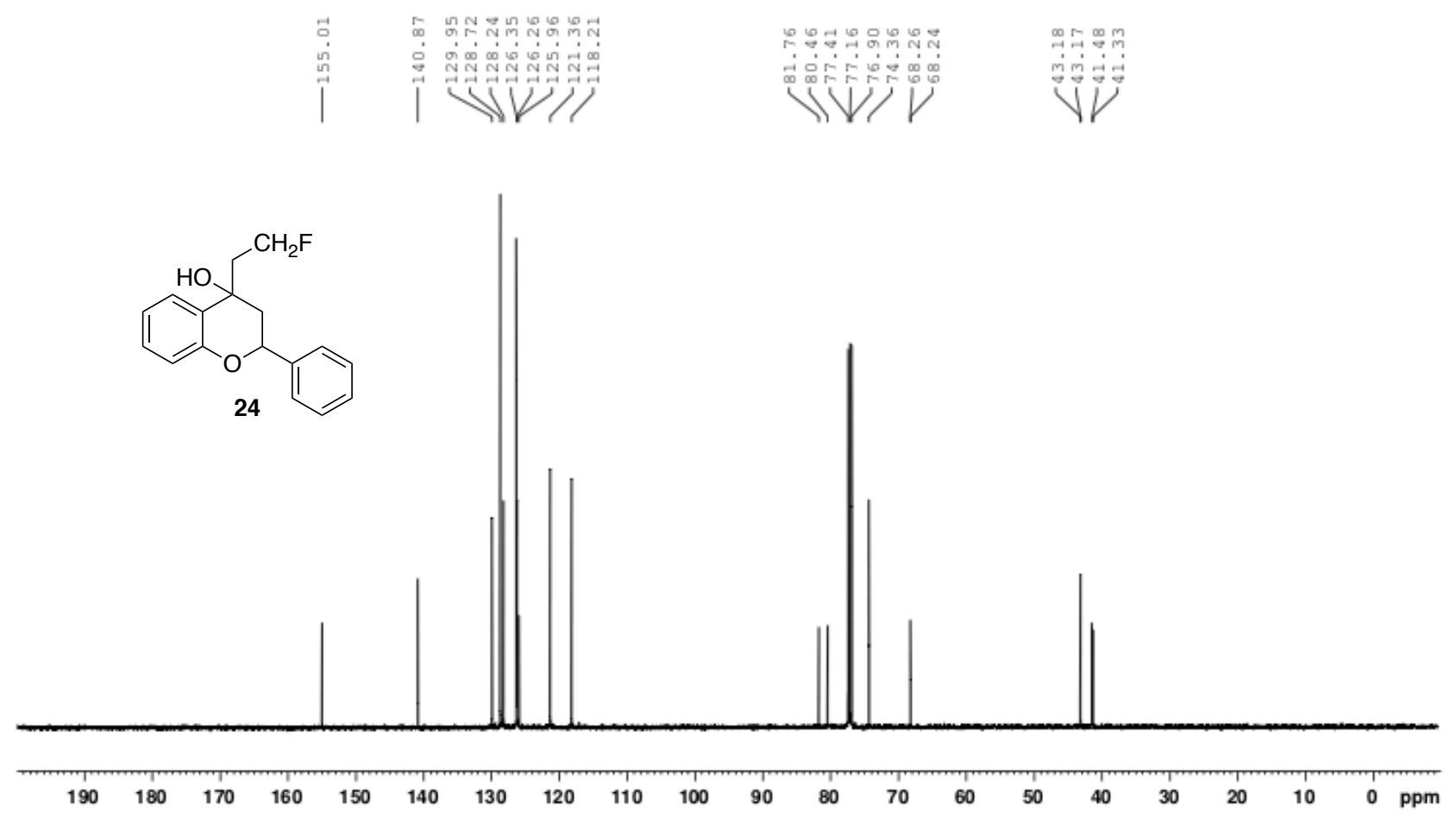

${ }^{19} \mathrm{~F} \mathrm{NMR}\left(376 \mathrm{MHz}, \mathrm{CDCl}_{3}, \mathrm{rt}\right)$

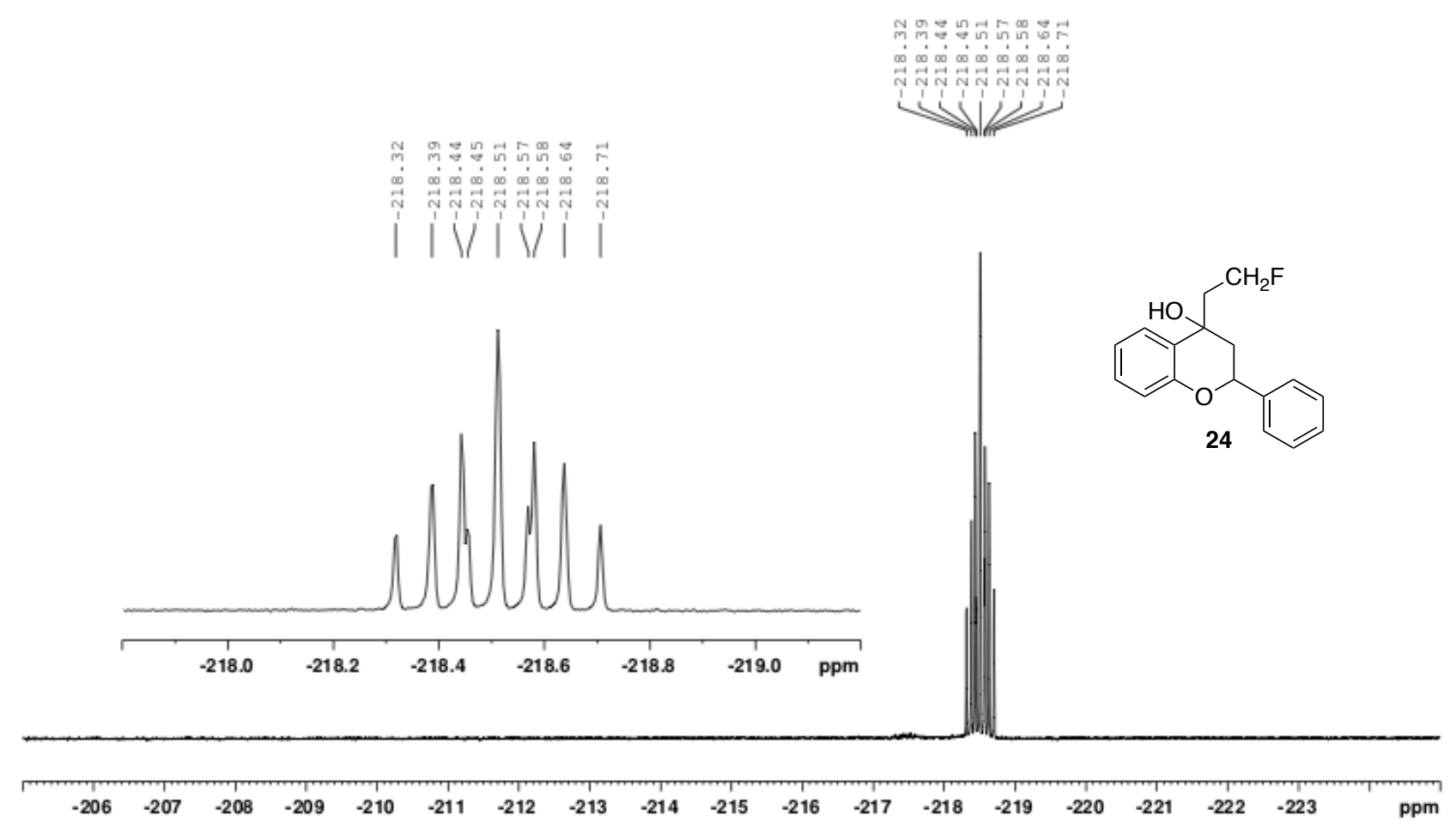


${ }^{1} \mathrm{H}$ NMR $\left(400 \mathrm{MHz}, \mathrm{CDCl}_{3}, \mathrm{rt}\right)$

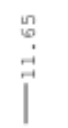

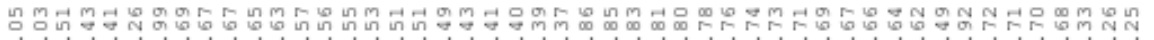

米
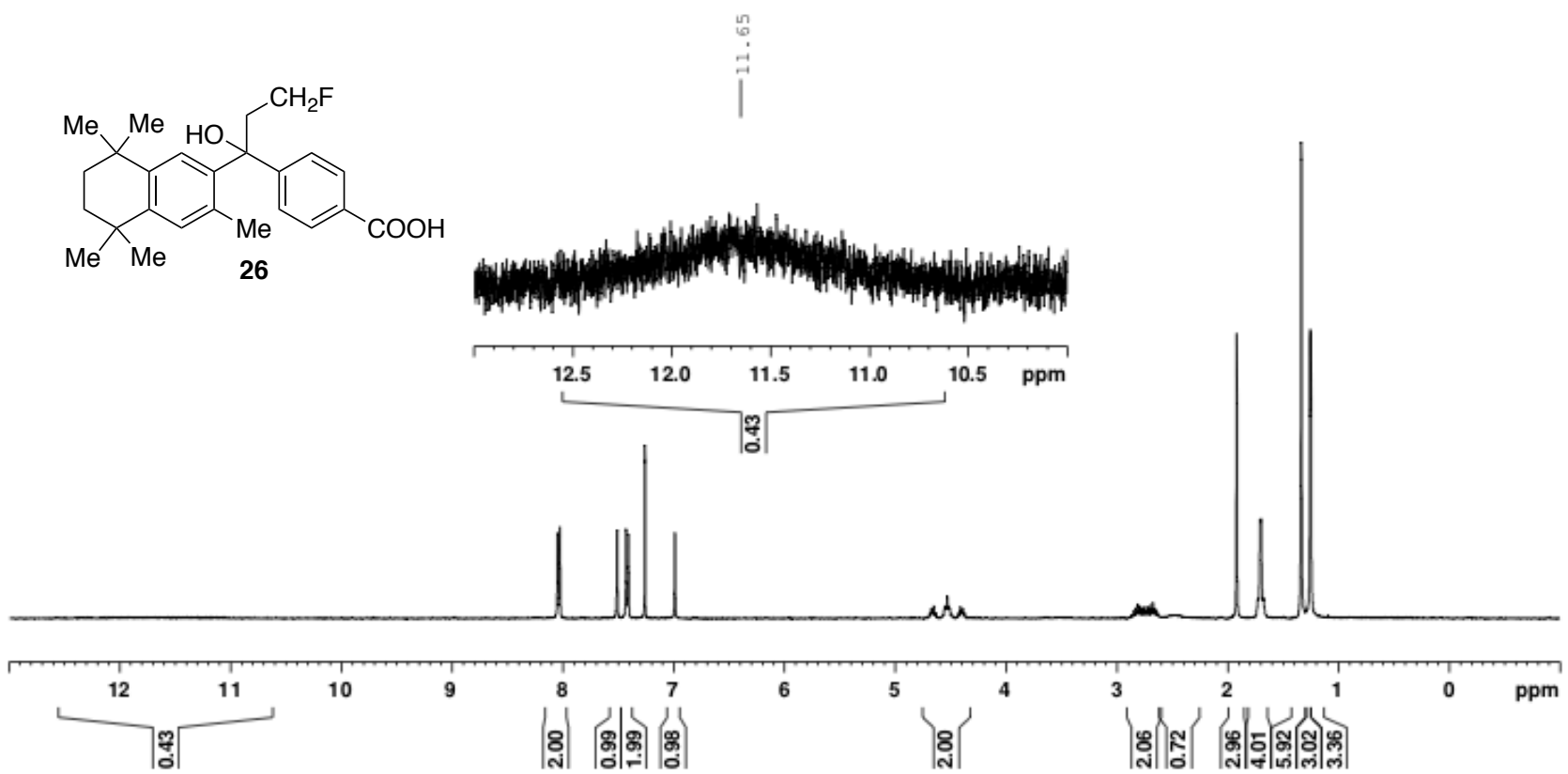

${ }^{13} \mathrm{C}$ NMR $\left(125 \mathrm{MHz}, \mathrm{CDCl}_{3}, \mathrm{rt}\right)$
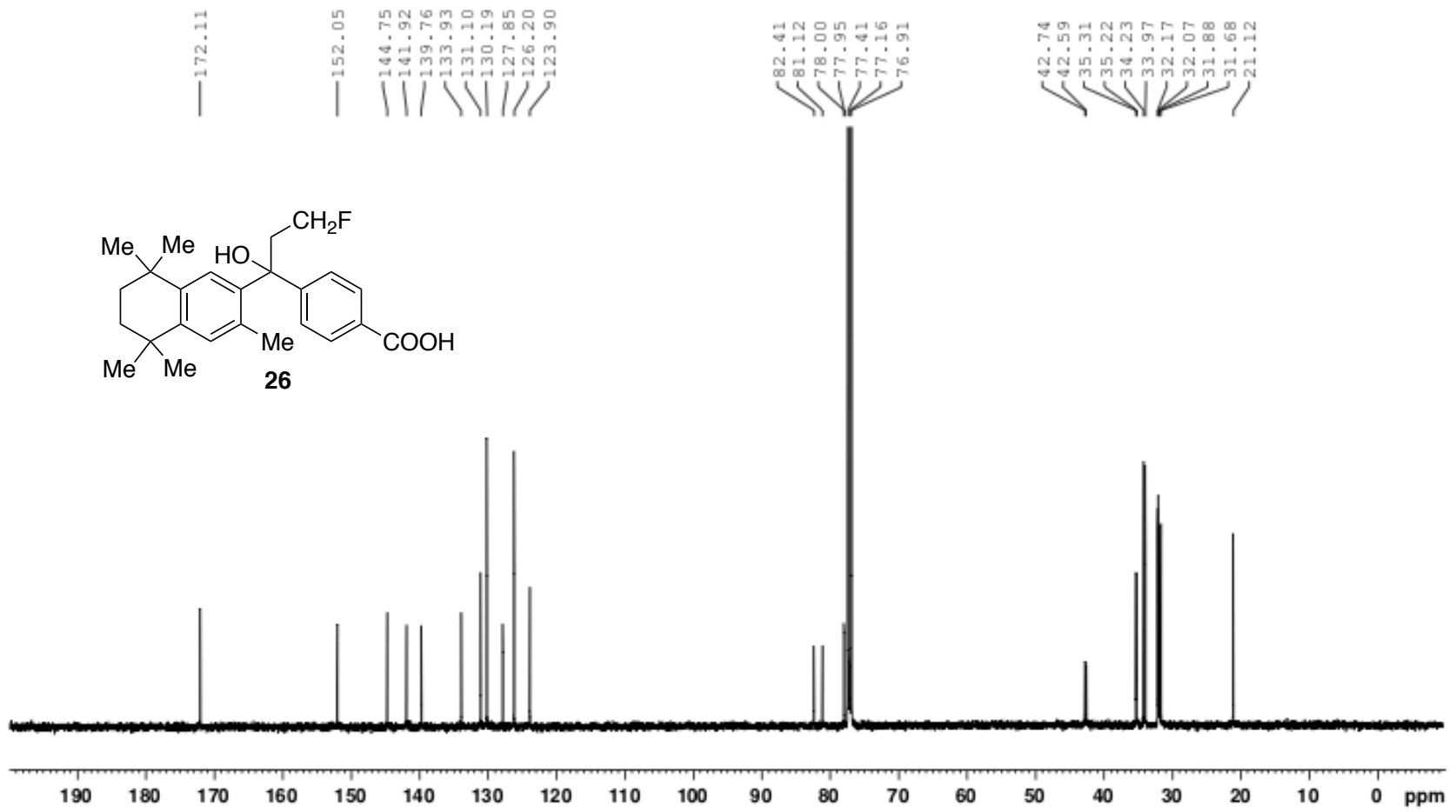
${ }^{19} \mathrm{~F} \mathrm{NMR}\left(376 \mathrm{MHz}, \mathrm{CDCl}_{3}, \mathrm{rt}\right)$

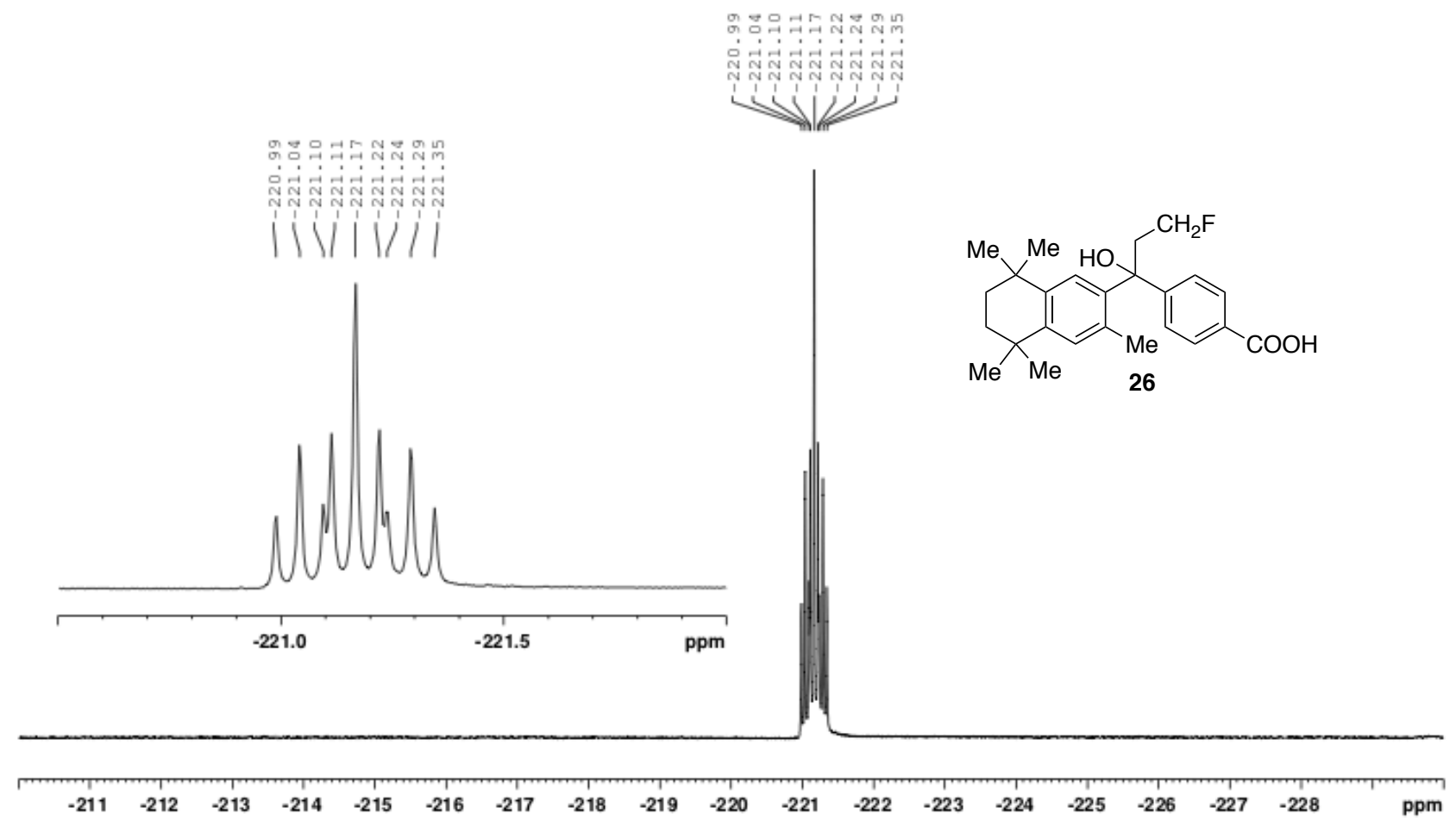

${ }^{1} \mathrm{H}$ NMR $\left(400 \mathrm{MHz}, \mathrm{CDCl}_{3}, \mathrm{rt}\right)$

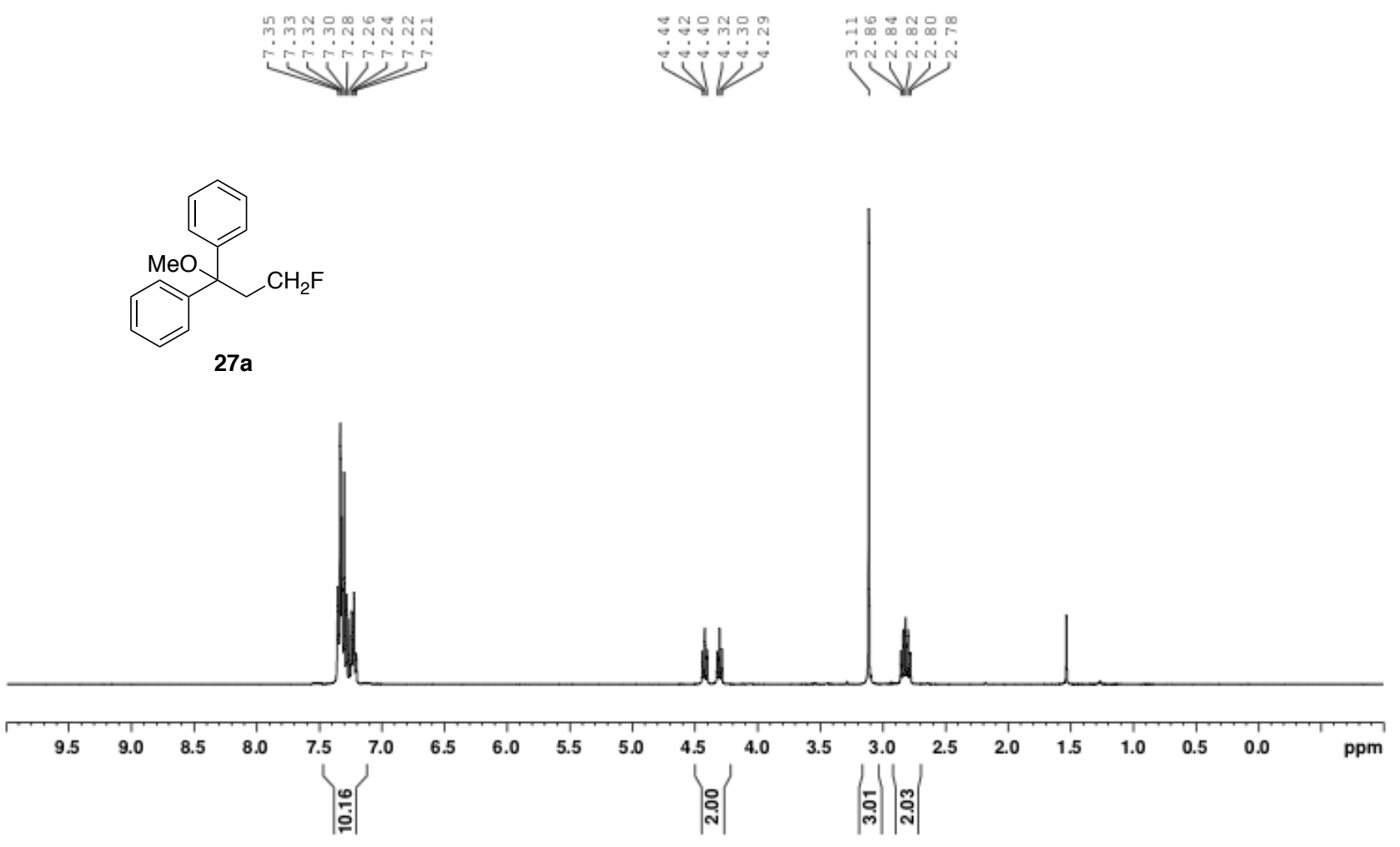


${ }^{13} \mathrm{C}$ NMR $\left(125 \mathrm{MHz}, \mathrm{CDCl}_{3}, \mathrm{rt}\right)$
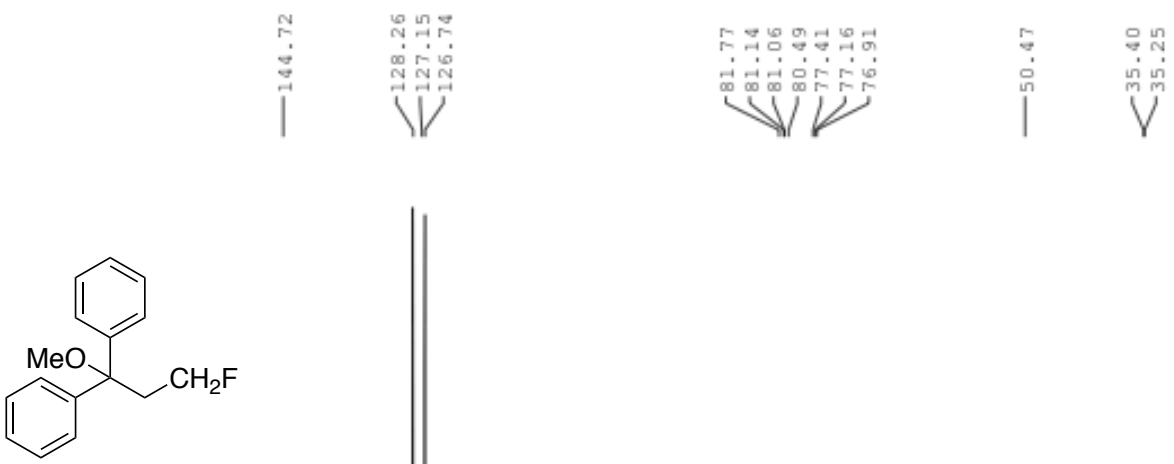

$27 a$

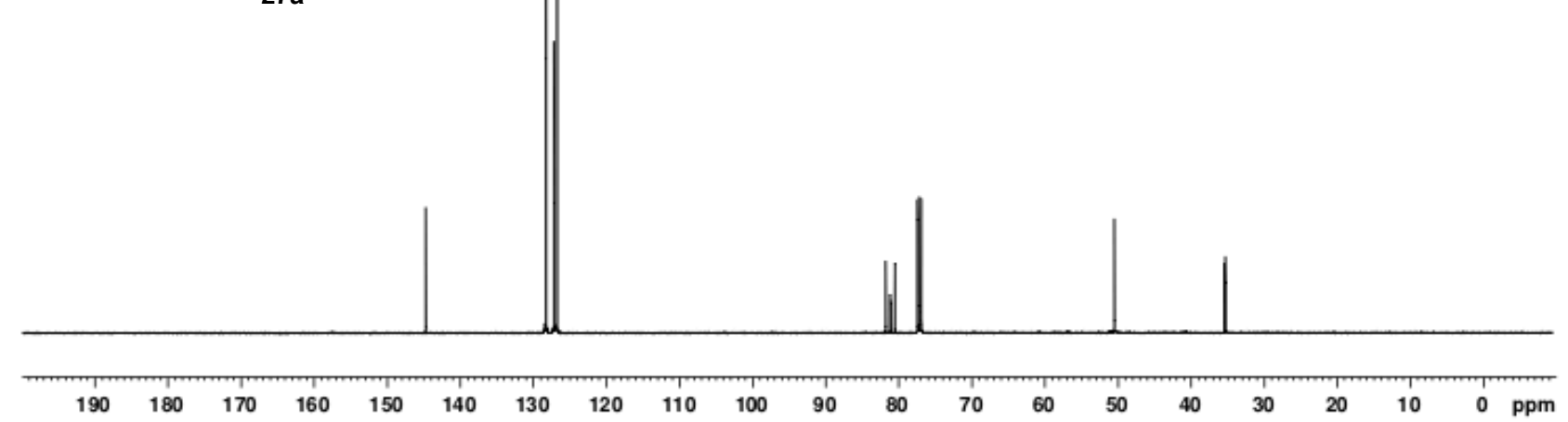

${ }^{19} \mathrm{~F} \mathrm{NMR}\left(376 \mathrm{MHz}, \mathrm{CDCl}_{3}, \mathrm{rt}\right)$

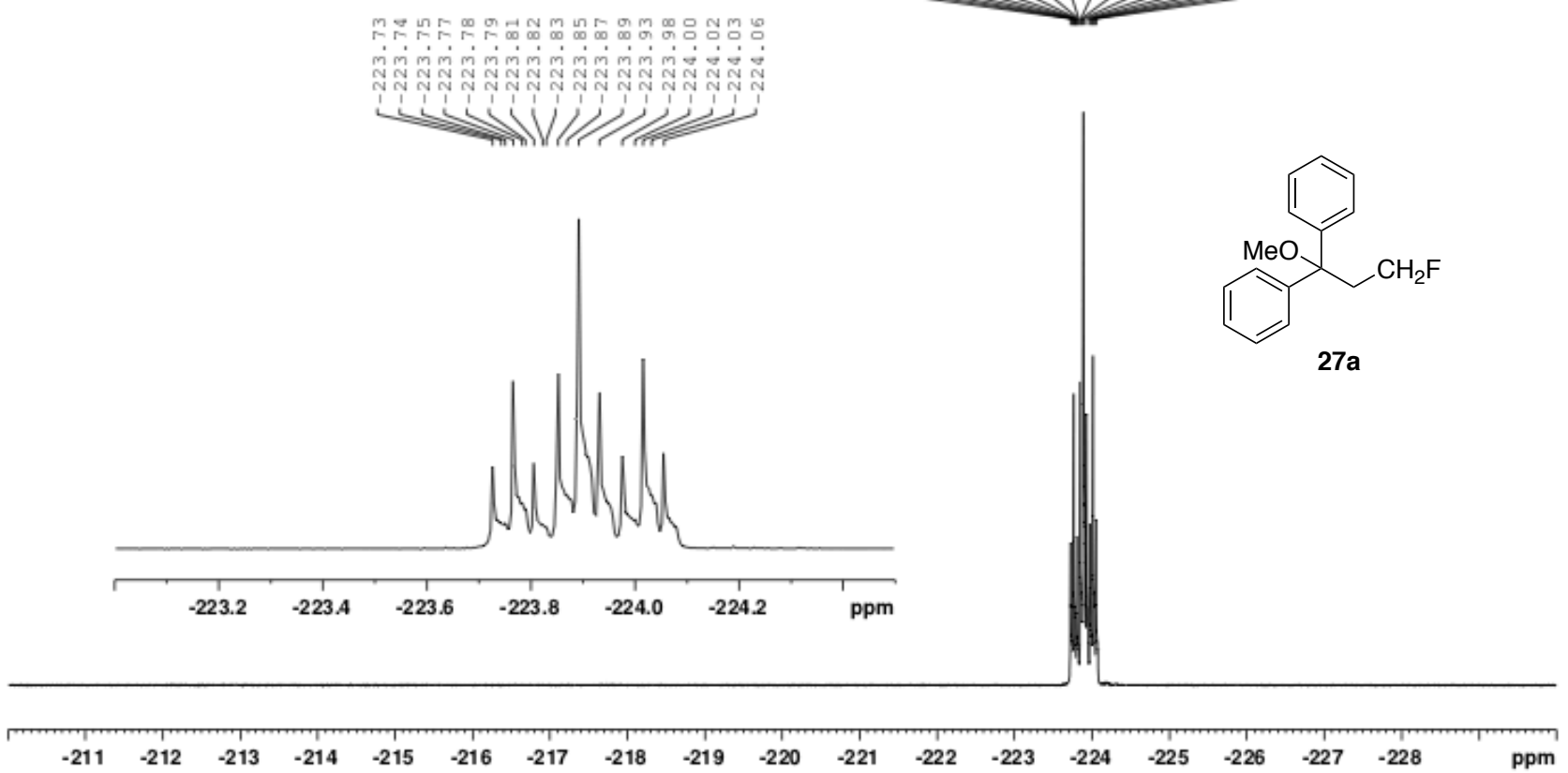


${ }^{1} \mathrm{H} \mathrm{NMR}\left(400 \mathrm{MHz}, \mathrm{CDCl}_{3}, \mathrm{rt}\right)$
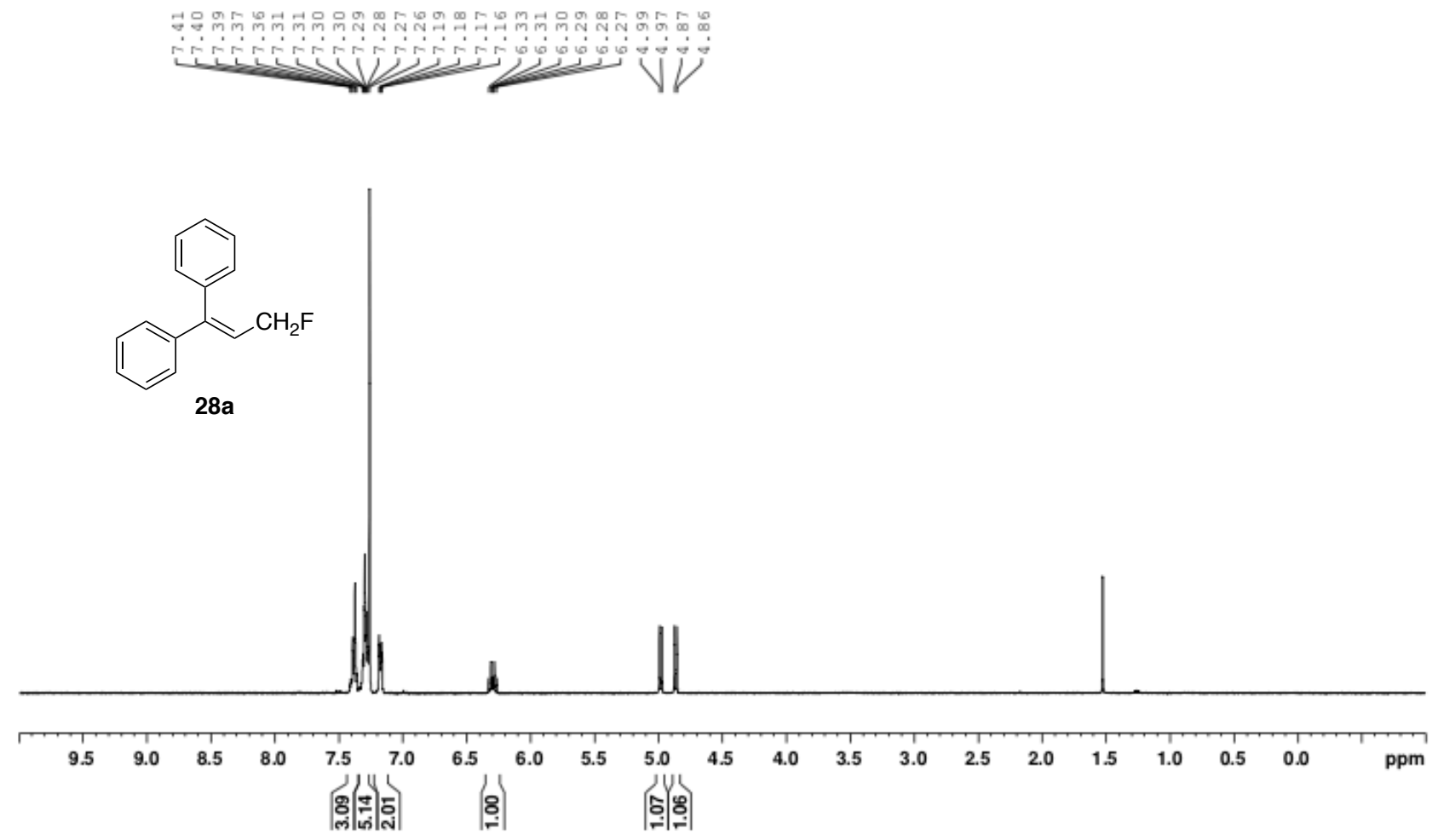

${ }^{13} \mathrm{C}$ NMR $\left(125 \mathrm{MHz}, \mathrm{CDCl}_{3}, \mathrm{rt}\right)$
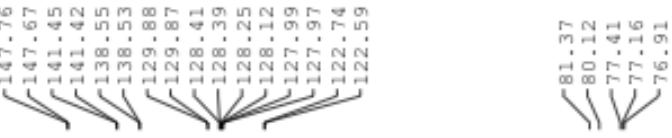<smiles>FCC=C(c1ccccc1)c1ccccc1</smiles>

$28 a$ 
${ }^{19} \mathrm{~F} \mathrm{NMR}\left(376 \mathrm{MHz}, \mathrm{CDCl}_{3}, \mathrm{rt}\right)$

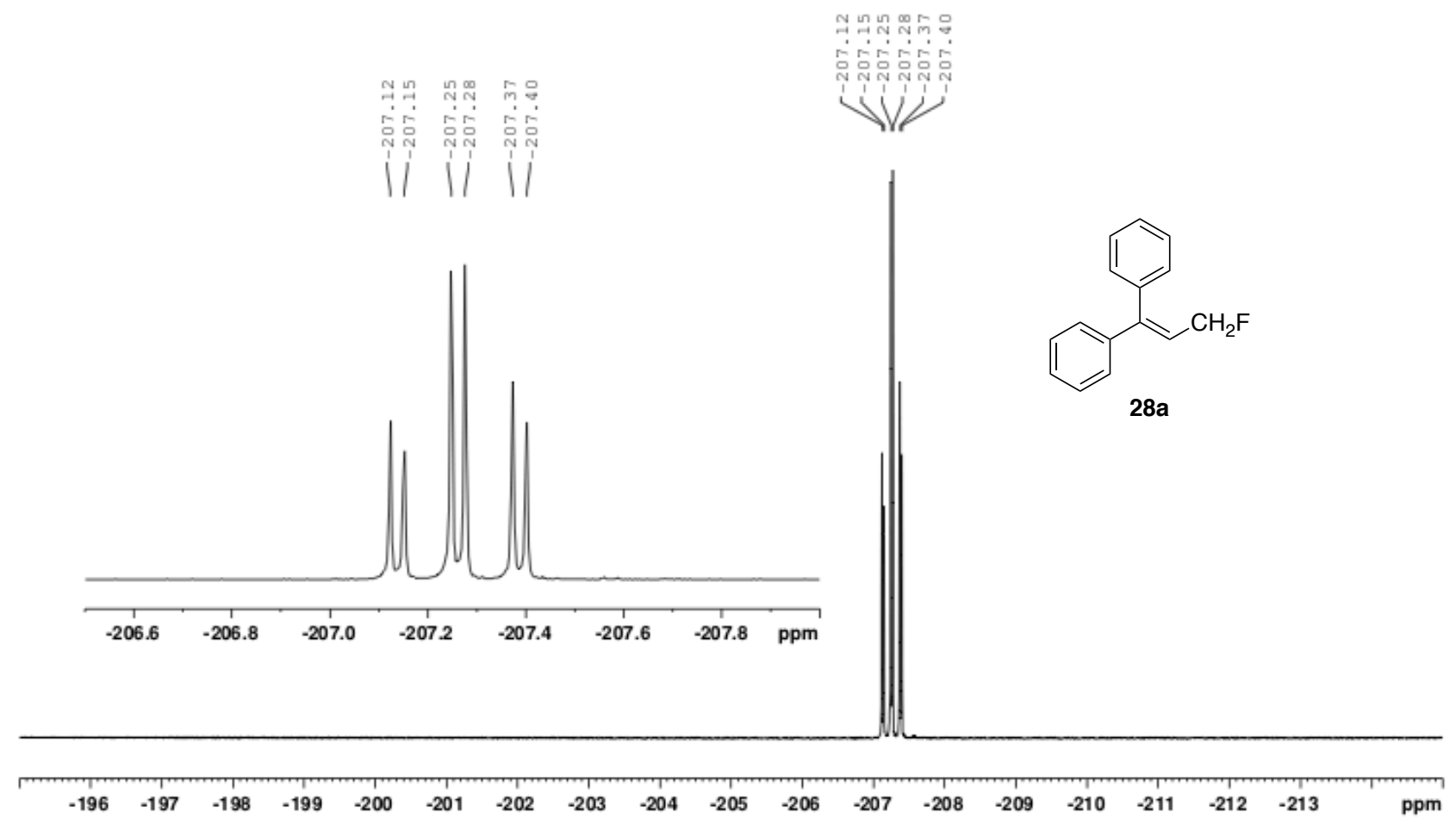

${ }^{1} \mathrm{H}$ NMR (400 MHz, $\left.\mathrm{CDCl}_{3}, \mathrm{rt}\right)$

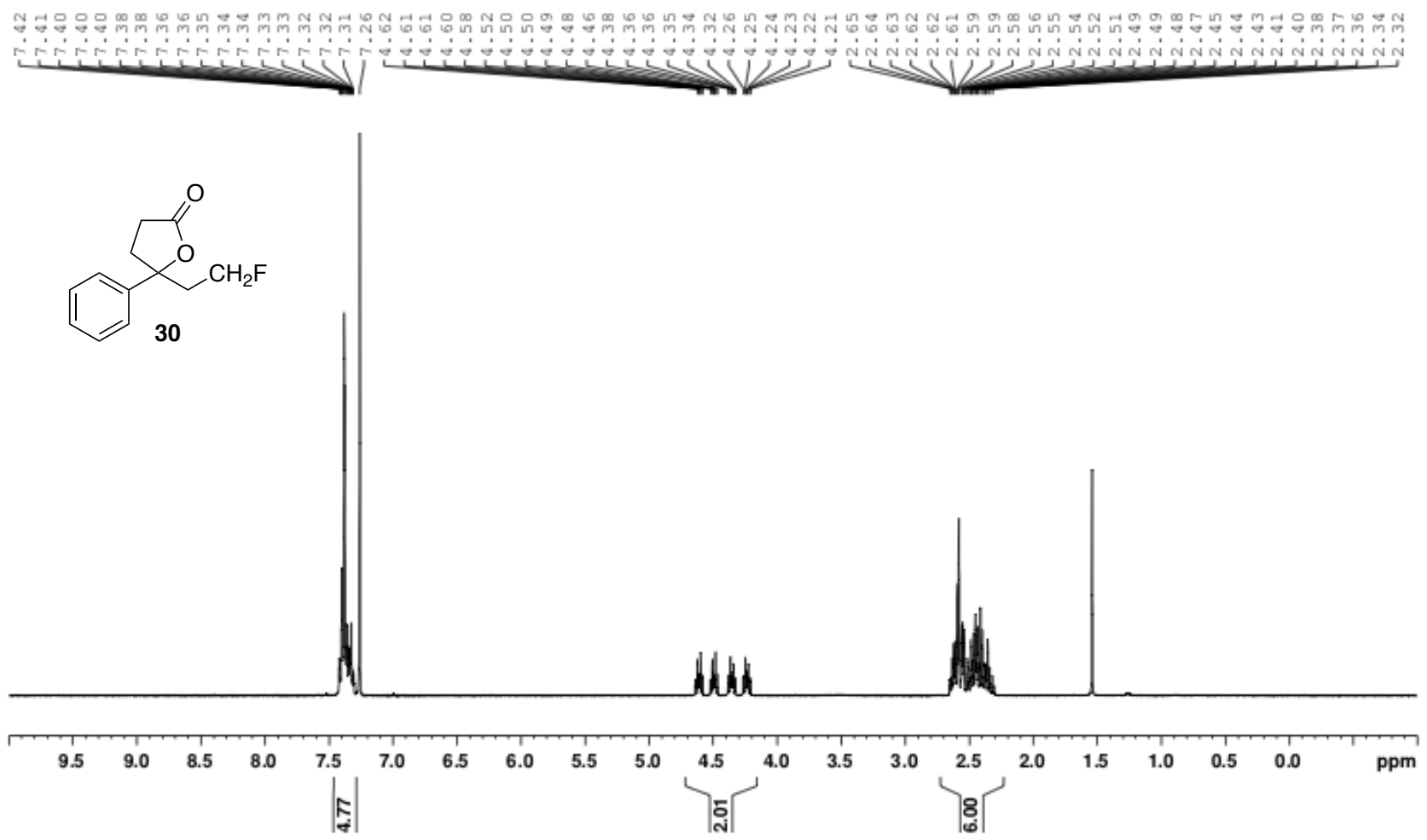


${ }^{13} \mathrm{C}$ NMR $\left(125 \mathrm{MHz}, \mathrm{CDCl}_{3}, \mathrm{rt}\right)$
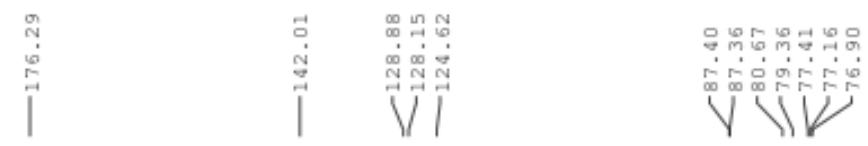

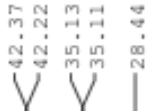
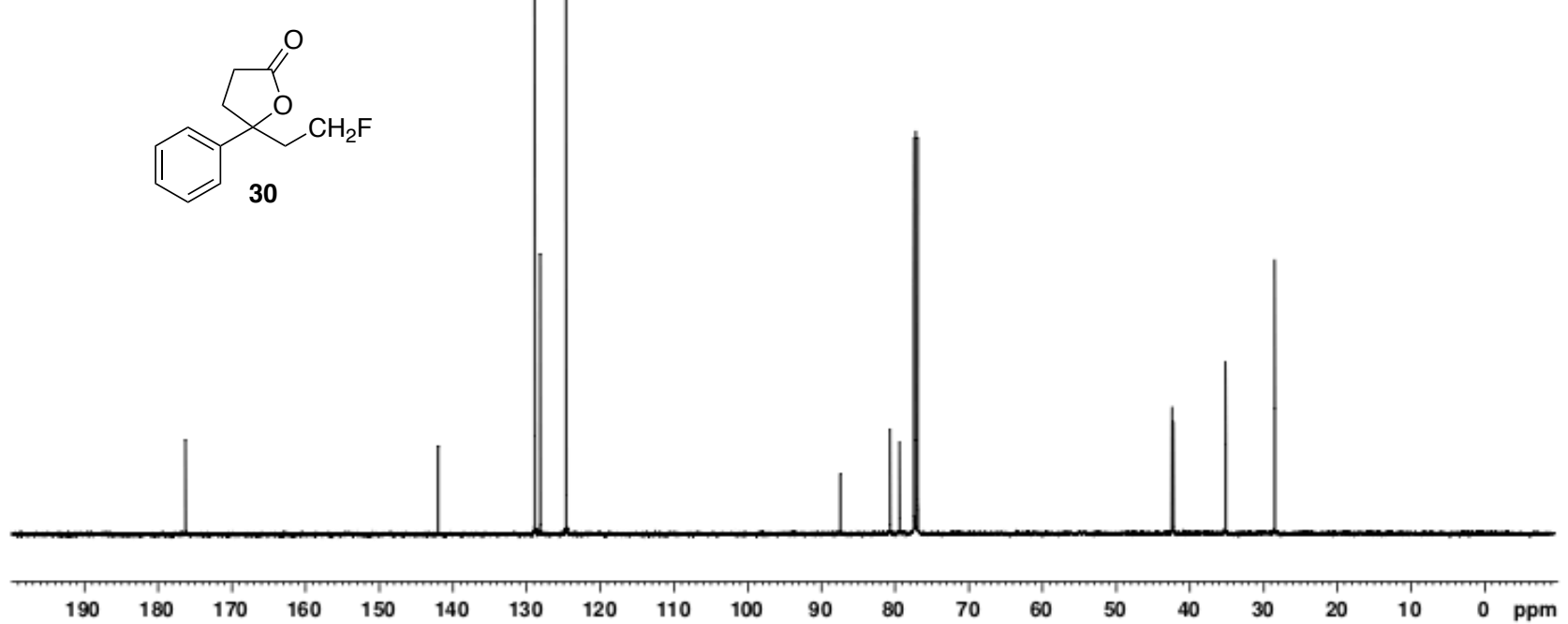

${ }^{19} \mathrm{~F} \mathrm{NMR}\left(376 \mathrm{MHz}, \mathrm{CDCl}_{3}, \mathrm{rt}\right)$

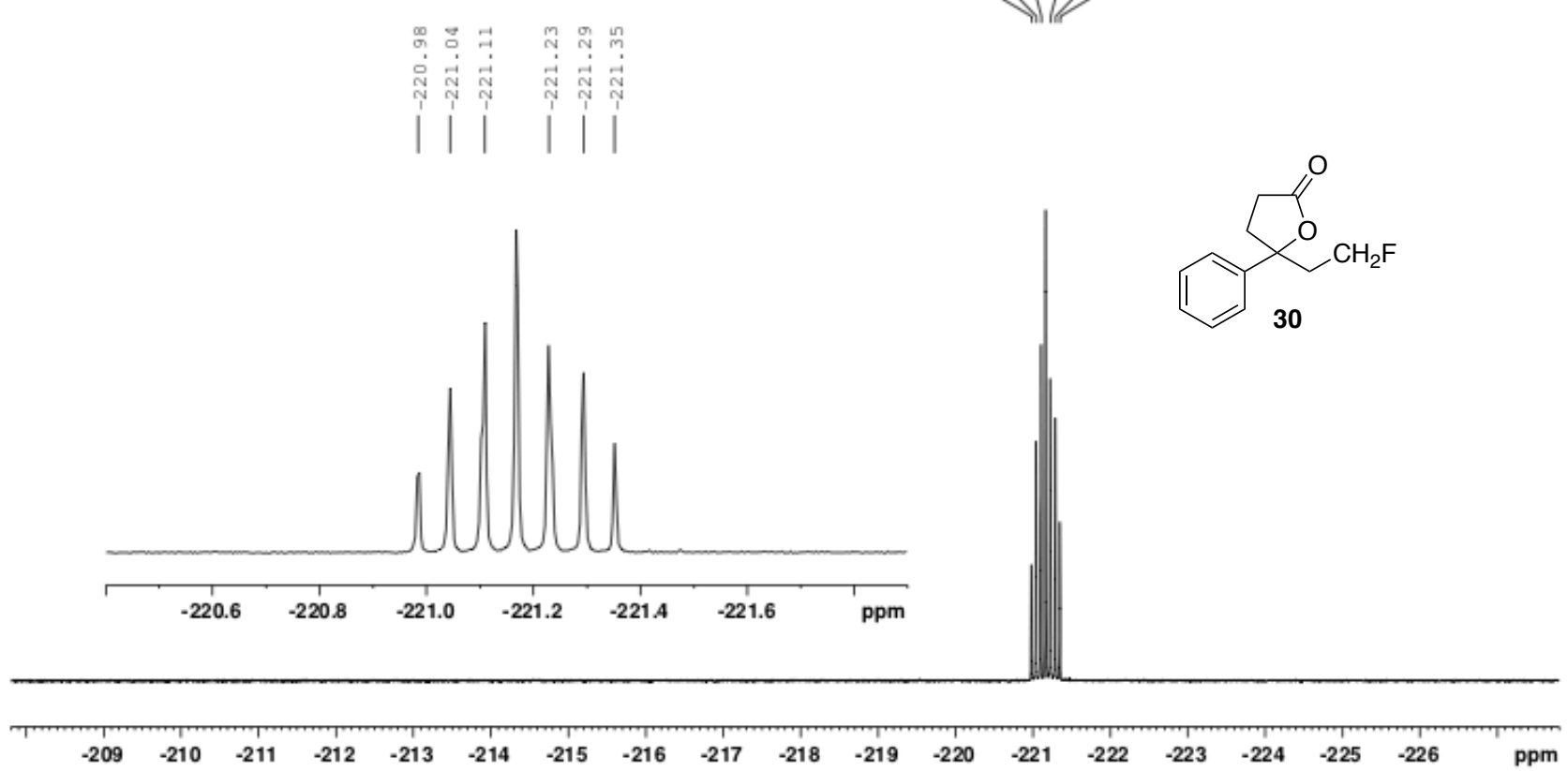

